Keeping track of the enemy

Flight analyses of the host-seeking malaria mosquito Anopheles gambiae s.s.

8

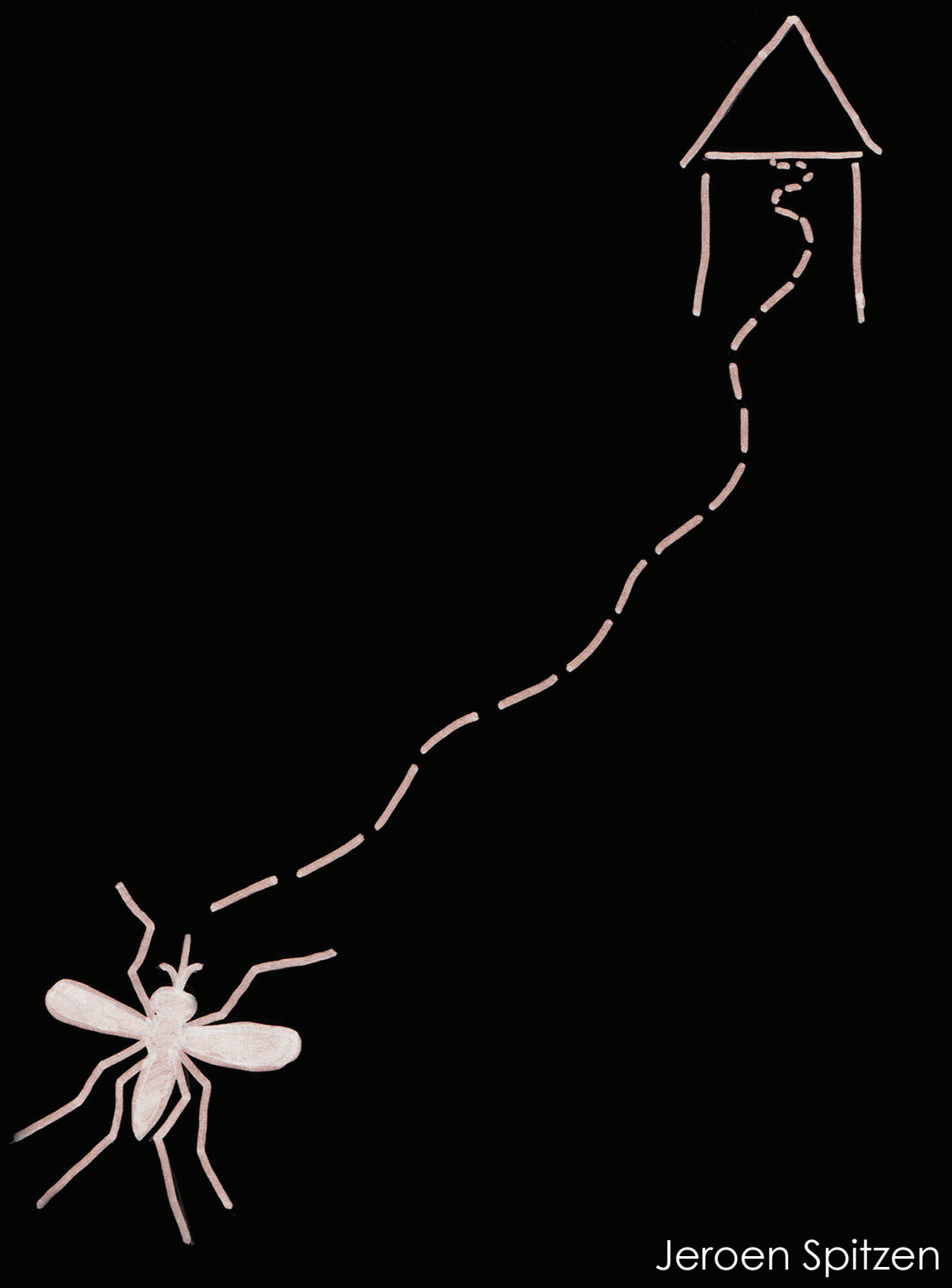




\section{Propositions}

1. The use of infrared light illuminates the behavioural ecology of nocturnal mosquitoes.

(this thesis)

2. Human odour causes prolonged and highly convoluted flight tracks and the combination with heat is crucial to induce landings of host-seeking mosquitoes. (this thesis)

3. The taboo on decreasing the global human population size should be lifted and needs to be addressed in a multifaceted approach on political and societal agendas.

4. Introduction of the 'polluter pays' principle will result in more environmental benefits than stimulating a growing market of organic and sustainable products.

5. Practice what you teach augments science for impact.

6. With the increase in number of PhD candidates, it becomes extremely challenging for the reviewing committee to check the originality of propositions.

Propositions belonging to the thesis, entitled

'Keeping track of the enemy:

Flight analyses of the host-seeking malaria mosquito Anopheles gambiae s.s.'

Jeroen Spitzen

Wageningen, 23 May 2018 


\section{Keeping track of the enemy}

Flight analyses of the host-seeking malaria mosquito Anopheles gambiae s.s.

Jeroen Spitzen 


\section{Thesis committee}

\section{Promotors}

Prof. Dr W. Takken

Personal chair at the Laboratory of Entomology

Wageningen University \& Research

Prof. Dr M. Dicke

Professor of Entomology

Wageningen University \& Research

\section{Other members}

Prof. Dr M. Naguib, Wageningen University \& Research

Dr F.O. Okumu, Ifakara Health Institute, Tanzania

Dr J.T. Bousema, Radboud University, Nijmegen, The Netherlands

Dr M.A.H. Braks, National Institute for Public Health and the Environment, Bilthoven, The Netherlands

This research was conducted under the auspices of the C.T. de Wit Graduate School for Production Ecology \& Resource Conservation. 


\title{
Keeping track of the enemy \\ Flight analyses of the host-seeking malaria mosquito Anopheles gambiae s.s.
}

\author{
Jeroen Spitzen
}

\section{Thesis}

submitted in fulfilment of the requirements for the degree of doctor at Wageningen University

by the authority of the Rector Magnificus,

Prof. Dr A.P.J. Mol, in the presence of the

Thesis Committee appointed by the Academic Board to be defended in public on Wednesday 23 May 2018 at 11 a.m. in the Aula. 
Jeroen Spitzen

Keeping track of the enemy: Flight analyses of the host-seeking malaria mosquito Anopheles gambiae s.s., 188 pages.

$\mathrm{PhD}$ thesis, Wageningen University, Wageningen, the Netherlands (2018) With references, with summaries in English and Dutch

ISBN 978-94-6343-258-0

DOI $10.18174 / 441165$ 



\section{Abstract}

Female mosquitoes can transmit pathogens to their host during blood feeding and are an important vector of human diseases such as dengue, chikungunya, filariasis and malaria. After 15 years of decline in the number of fatal malaria cases, this decline came to a halt in 2016. Growing resistance against drugs and insecticides pose a serious threat for future human health.

This thesis focuses on the behaviour of host-seeking malaria mosquitoes by analysing their flight paths during their approach to different host cues. Fundamental knowledge on the role of selected host cues was acquired. In addition, studies to support successful implementation of vector control interventions were performed in both windtunnel settings and the semi-field in Kenya. My research demonstrates that automated tracking systems can strengthen behavioural-ecological studies on disease vectors, in addition to conventional bio-assays such as olfactometers, by providing detailed information on the approach behaviour of mosquitoes to different targets.

The attraction towards the host-sensory cue $\mathrm{CO}_{2}$ was investigated in an olfactometer bioassay. Trap catches of female Anopheles gambiae s.s. were enhanced by separation of the $\mathrm{CO}_{2}$ source from the source of human skin emanations. Close-range deterrent effects of $\mathrm{CO}_{2}$ were overcome by the simultaneous presence of skin emanations. Flight path analysis of mosquitoes in a wind tunnel, showed that exposure to human odour resulted in prolonged and highly convoluted flight tracks. The combination of odour with heat was crucial to induce landings of host-seeking mosquitoes. A semifield study in Kenya revealed that house-entering mosquitoes approached the eave of a house in a wide angle to the house at eave level, where the proportion that entered uninterruptedly (23\%) spent just a few seconds around the eave area. The presence of insecticide-treated nets inside a house did not repel mosquitoes as measured by the number of house entries. At close range, in a wind tunnel, free-flight exposure of mosquitoes to deltamethrin-treated nets in combination with human odour did not reveal any (excito-) repellent effect and resulted in lower mortality rates compared to standard bioassays where contact with the treated material is enforced.

The knowledge obtained on the behavioural responses of mosquitoes to host cues has indirectly affected vector control tool implementations in the field. For example,

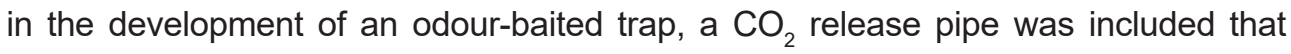
is separated from the attractive odour plume. The role of heat was exploited in the development of a repellent bioassay and a heat source was added to another trap model. Insights in house-entry behaviour and mosquito responses to bed nets support the successful implementation of push-pull systems, installation of eave tubes or implementing house improvement operations to reduce malaria transmission. An integrated vector management approach is required to further develop existing control tools by adding and improving alternative intervention techniques. 


\section{Table of Contents}

$\begin{array}{ll}\text { Abstract } & 6\end{array}$

Chapter $1 \quad 9$

General Introduction

Chapter 2

Keeping track of mosquitoes: A review of tools to track, record and analyse mosquito flight

\section{Chapter 3}

Effect of human odours and positioning of $\mathrm{CO}_{2}$ release point on trap catches of the malaria mosquito Anopheles gambiae sensu stricto in an olfactometer

\section{Chapter 4}

A 3D analysis of flight behaviour of Anopheles gambiae sensu stricto malaria mosquitoes in response to human odour and heat

\section{Chapter 5}

Visualization of house-entry behaviour of malaria mosquitoes

\section{Chapter 6}

Effect of insecticide-treated bed nets on house-entry by malaria mosquitoes: The flight response recorded in a semi-field study in Kenya

\section{Chapter 7}

Absence of close-range excitorepellent effects in malaria mosquitoes exposed to deltamethrin-treated bed nets

\section{Chapter 8}

General Discussion

References

Summary

Samenvatting

Acknowledgements

Curriculum Vitae

Education statement 
(

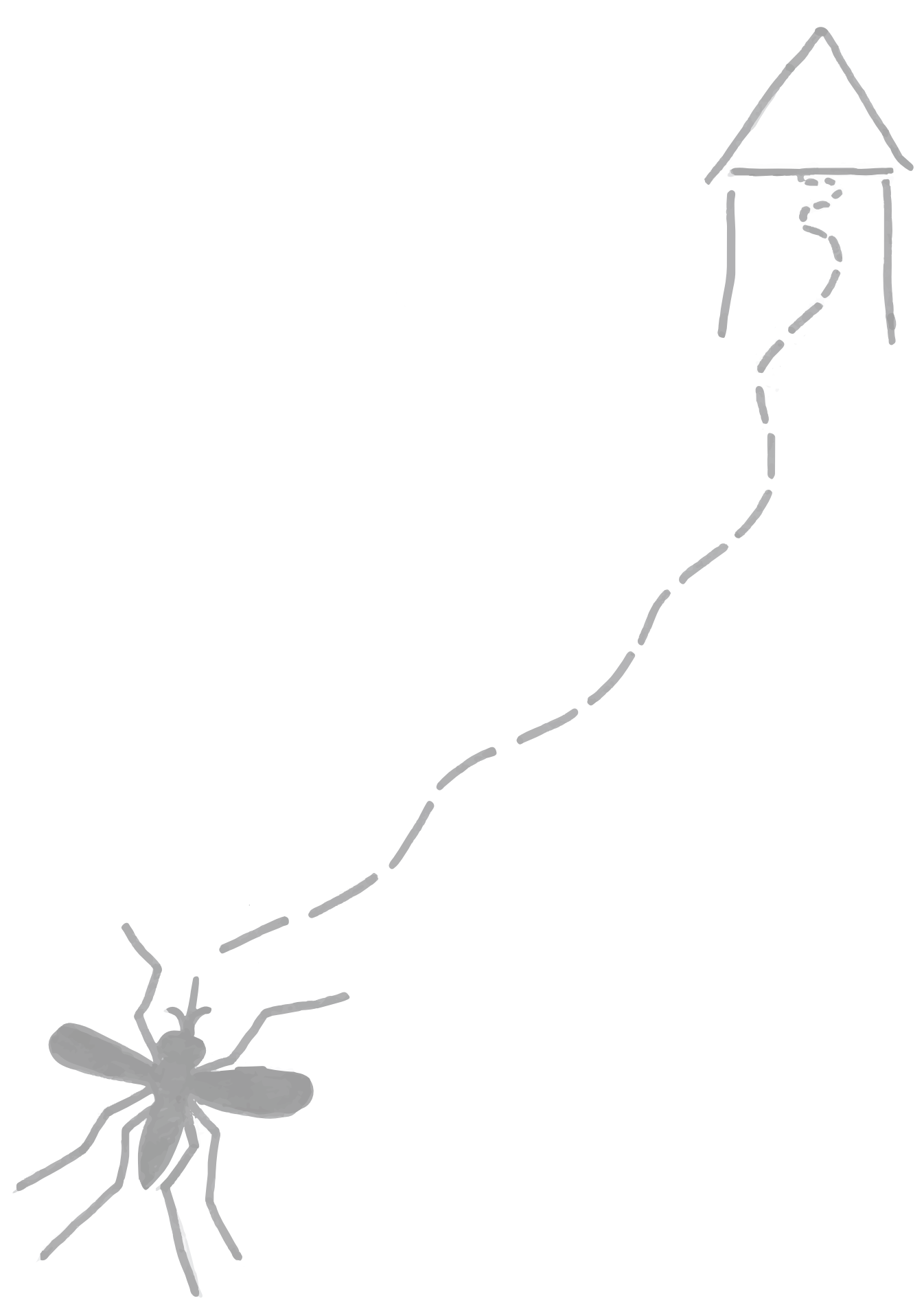




\section{Chapter 1}

General Introduction 


\section{Chapter 1}

\section{Introduction}

Blood-sucking arthropods cause nuisance because their bites can hurt and cause itching. The sound of an approaching mosquito in flight induces agitation of the host target. Many of them transmit pathogens that cause serious health problems across the world. It is this indirect effect why mosquitoes are considered the deadliest animals of planet earth. Among the mosquitoes that act as vectors of infectious diseases such as dengue, yellow fever, Zika or West-Nile virus, the genus Anopheles, with 465 species and over 50 unnamed members of species complexes, compiles 41 species considered dominant in transmitting the Plasmodium parasite to humans (Sinka et al., 2012 and references therein). This group caused an estimated number of 216 million human malaria cases in 2016, with approximately 445,000 deaths worldwide (World Health Organization, 2017a). Vector-control tools such as longlasting-insecticide-treated nets (LLINs) and indoor residual spraying (IRS) currently lose their efficacy due to behavioural and physiological resistance (N'Guessan et al., 2007; Ranson et al., 2011). Alternative control methods are required. Many of these methods aim to interrupt mosquito-host interactions, e.g. by the use of odour-baited traps, push-pull systems, or house improvements (Homan et al., 2016; Menger et al., 2016; Tusting et al., 2016). For the innovation and successful implementation of alternative, sustainable tools it is necessary to understand mosquito behaviour in detail so that interventions can be made more precisely and effectively.

This thesis focuses on the flight behaviour of one of the main vectors of malaria parasites, Anopheles gambiae Giles sensu stricto, while exposed to host cues. Automated tracking techniques were developed and used to unravel flight tracks of nocturnal mosquitoes. Fundamental knowledge on the role of specific host cues was acquired and applied studies to support successful implementation of vector control interventions were performed in both wind-tunnel settings or the semi-field in Kenya.

\section{Malaria}

Malaria is caused by protozoa of the genus Plasmodium. The parasite is transmitted to humans through bites of infected female mosquitoes belonging to the genus Anopheles (Diptera: Culicidae). Plasmodium falciparum is the main species causing (fatal) malaria and is dominant in Sub-Saharan Africa, while $P$. vivax is the dominant species in Asia and South America, although with fewer cases than $P$. falciparum (Vogel, 2013). The other three species that can cause human malaria are $P$. ovale, P. knowlesi and P. malariae (White et al., 2014).

The parasite needs both humans and mosquitoes for its development. As a result of a mosquito bite, sporozoites travel via the saliva of an infected mosquito to the liver 
of their host. Here, merozoites are produced which can infect red blood cells. Within these blood cells, asexual reproduction of the parasites takes place till they burst. As a result, the patient suffers from anaemia and the defence response (fever, nausea) against the toxic 'waste' factors that have occupied the bloodstream. Some parasites develop into gametocytes which in turn, can be taken up by female mosquitoes when they ingest a blood meal. Sexual reproduction of the parasite continues inside the midgut of the mosquito producing sporozoites that migrate to the salivary glands (White et al., 2014). In short, female mosquitoes can only infect new hosts with malaria parasites from their second blood meal onwards, given that there is sufficient time (10-18 days) between the initial infectious bite and the second bite (Aly et al., 2009).

\section{Mosquitoes}

Malaria parasite transmission is dominated by a relatively small group of the 465 named Anopheles species. Their vector capacity is driven by both genetic variation in the susceptibility to Plasmodium infections and habitat preference. The latter typically coincides with their anthropophilic and endophilic behaviours (Takken \& Verhulst, 2013; White et al., 2011). The species complex of Anopheles gambiae consists of eight sibling species and is mostly active in sub-Saharan Africa: Anopheles gambiae, An. coluzzii, An. arabiensis, An. quadriannulatus, An. amharicus, An. melas, An. merus and An. bwambae. The highly anthropophilic member Anopheles gambiae s.s. was recently assigned a new name for the molecular $\mathrm{M}$ - and $\mathrm{S}$ form where the $\mathrm{M}$ form is now referred to as $A n$. coluzzii and the $S$ form as An. gambiae (Coetzee et al., 2013). Outside the An. gambiae complex, An. funestus is also considered an important malaria vector (Sinka et al., 2010). Within this thesis, An. coluzzii was used for all laboratory experiments performed in The Netherlands and An. gambiae s.s. was used for the semi-field work in Kenya. I have not found published reference that the two forms differ in behaviour towards host cues, and therefore have assumed that their host specificity is similar, and that they respond equally to host cues (Lehmann \& Diabate, 2008). Because this thesis contains (published) data that was collected before the subdivision of An. gambiae s.s. was made, I refer to this name throughout the thesis to avoid confusion.

\section{Life cycle in brief}

Newly-emerged adult mosquitoes, especially the males, will stock up on plant sugar sources for further maturation of their reproductive organs (Clements, 1992). Mating occurs in swarms, usually before females haven taken their first blood meal, and sperm can be stored in the female spermatheca, sufficient to fertilize multiple egg batches without the need for further mating between gonotrophic cycles. An. 


\section{Chapter 1}

gambiae s.s. females require a minimum of one blood meal per gonotrophic cycle to develop eggs. The number of blood meals needed is correlated to adult size and related to metabolic reserves and pathogen infections (Briegel \& Horler, 1993; Koella et al., 1998; Scott \& Takken, 2012). After maturation of the eggs, mosquitoes search for a suitable oviposition site to deposit the eggs. For An. gambiae, typically small still-standing water bodies, animal hoof prints or car tracks filled with water or on a larger scale, rice fields, form suitable habitats for the larvae with low risk of predation. Larvae feed on aquatic microorganisms and small particles of decayed plant remains (Clements, 1999). The larvae moult four times before developing into a pupa that remains aquatic and mobile, but does not feed anymore. Under tropical conditions, a new generation can emerge as soon as eight days after egg laying where development is strongly related to temperature, competition for space and food (Farjana et al., 2011; Grigaltchik et al., 2016).

\section{Control}

Global efforts have led to an impressive reduction in the burden of malaria, which was accelerated by the founding of the Roll Back Malaria (RBM) Partnership in 1998. The first global malaria action plan, that served from 2008-2015, achieved a great deal of its goals with a reduction of ca. $50 \%$ of fatal cases. This achievement was a result of implementation of rapid diagnostic tests in combination with artemisininbased combination treatments next to large scale usage of LLINs and IRS (Bhatt et al., 2015; WHO-RBM, 2015). Increased reports of resistance against both drugs and insecticides created awareness to search and implement alternative control measures in order to reach the targets for the second action plan: 'Action and investment to defeat malaria 2016-2030, for a malaria-free world' (WHO-RBM, 2015). The plan fits under the umbrella of a new WHO Global Vector Control Response (GVCR) programme that presents sustainable measures against other vector-borne diseases as well (Alonso et al., 2017; World Health Organization, 2017b).

Wageningen University and Research has been involved in research and implementation of vector control tools that match with Integrated Vector Management (IVM) programmes for decades. The tools aim at endured efficacy without the need of insecticides. An example is the use of entomopathogenic fungi applied in or around houses where it can reduce malaria transmission with $75 \%$ (Scholte et al., 2005). Another approach is to exploit push-pull principles by the use of repellent (push) and attractive (pull) cues positioned strategically on host-seeking routes. The approach is successful in agricultural systems (Khan et al., 2011), but recently showed its potential to reduce malaria transmission when tested in field settings (Homan et al., 2016; Menger et al., 2015). Where projects that focus on house improvement show a reduction in indoor malaria transmission, the addition of e.g. odour-baited 
traps placed outdoors can reduce (remaining) outdoor transmission (Killeen, 2014; Tusting et al., 2016). Following the IVM approach, the above measures are preceded by larval source management actions. Removing larval habitats, or applications with a biopesticide like Bacillus thuringiensis israelensis (World Health Organization, 2012). The four approaches mentioned have been illustrated in a field setting (Figure 1 ) and can be easily remembered following the acronym HELP.

\section{Mosquito flight behaviour}

Mosquitoes rely on efficient flight strategies throughout their imago stage. To successfully reproduce, they have to locate energy resources, a partner to mate, a blood source to develop eggs and an oviposition site to deposit them. As with many insect species, mosquitoes use odour cues to identify and locate their target site. However, other cues such as visual cues, heat or wind direction, also affect successful source location (Cardé \& Gibson, 2010; Cardé \& Willis, 2008). The relative importance of each cue is highly species specific. The important role of carbon dioxide $\left(\mathrm{CO}_{2}\right)$ for example, has been recognized for decades (Gillies, 1980; Rudolfs, 1922) and is different between mosquito species, depending on their host preferences (Dekker \& Takken, 1998; Essen et al., 1994). The interplay with other

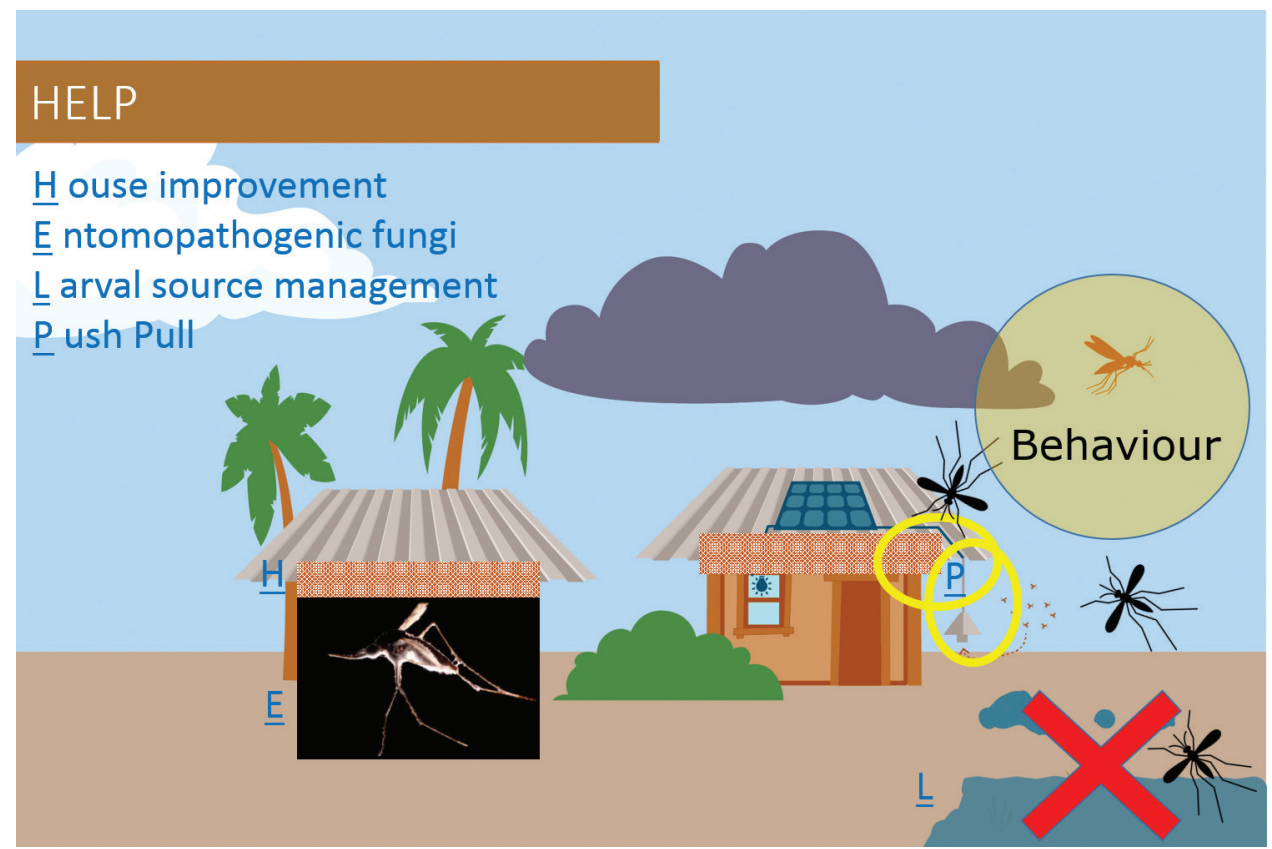

Figure 1. Infographic modified after Ties Blaauw, Communication Services, Wageningen University and Research. Successful development and implementation of vector control tools relies on detailed understanding of mosquito behavioural ecology. Picture of fungus-infected mosquito by Hugh Sturrock. 


\section{Chapter 1}

stimuli and temporal responses towards $\mathrm{CO}_{2}$ is still being explored (McMeniman et al., 2014; Webster et al., 2015). Visual cues also play a different role for diurnal mosquitoes than for nocturnal species (Gibson, 1995; Muir et al., 1992). At last, An. gambiae s.s. typically shows upwind host-seeking behaviour, even in the absence of odour cues, which seems a different strategy to locate hosts compared to other species like Aedes aegypti (reviewed by Cardé \& Gibson, 2010).

Bio-assays using dual-choice olfactometers, traps, semi-field containment facilities, have contributed to our understanding of (host-) preferences of mosquitoes and the role of specific target site cues (Butler, 2007; Takken \& Verhulst, 2013). However, less is known about the approach towards these targets. Descriptions of flight behaviour benefit our knowledge on mosquito behavioural ecology and with that, vector-intervention tools such as odour-baited traps or house improvements can be further developed and optimized (Figure 1). Understanding the integration of different cues and how they affect the approach of mosquitoes to reach their target requires more detailed analyses of individual flight tracks (Cardé, 2015; Cardé \& Willis, 2008; McMeniman et al., 2014). Mosquito flight patterns are typically 3 dimensional (3D) and as such, 2 dimensional (2D) analyses cannot reveal their full behavioural repertoire (Cardé \& Gibson, 2010; Rutkowski et al., 2009).

\section{Aim of this thesis}

The aim of this thesis was to gain fundamental knowledge on how An. gambiae s.s. mosquitoes respond to a combination of host cues, in particular skin emanations, $\mathrm{CO}_{2}$ and heat. An additional goal was to demonstrate that behavioural-ecological studies on disease vectors can benefit from automated tracking systems, next to conventional bio-assays such as olfactometers, by providing detailed information on the approach of mosquitoes to different targets. To accomplish this objective, a $3 \mathrm{D}$-tracking system was developed that was used in both laboratory and semi-field settings.

\section{Thesis outline}

Recent advances in tracking techniques have opened a new array of possibilities to monitor, measure and manipulate mosquito flight behaviour. In Chapter 2, developments in mosquito tracking techniques are reviewed and the applications for mosquito vector ecology research are discussed.

$\mathrm{CO}_{2}$ plays a key-role in host finding by most haematophagous arthropods. The role of $\mathrm{CO}_{2}$ during host-seeking of mosquitoes has been studied over many years, but its function is different between mosquito species and there are still some open 
questions on its exact role in flight behaviour at certain distances from the source, i.e. the host. Chapter 3 describes a dual-choice assay, where we built on the fact that despite mosquito attraction to $\mathrm{CO}_{2}$, they do not fly into a person's mouth. We address the crucial role of positioning of $\mathrm{CO}_{2}$ sources in combination with (synthetic) skin emanations in order to lure malaria mosquitoes into traps.

More detailed information on host finding and underlying strategies to follow host cues can be studied in wind-tunnel setups equipped with behaviour-recording software. In Chapter 4 the development of such a recording set-up is described and the role of heat in addition to skin emanations during host seeking is investigated.

Whereas laboratory studies can provide high quality data on specific flight behaviours, successful implementation of vector control tools relies on the manipulation of behaviour of mosquitoes that are exposed to a broader odour palette under field conditions. A semi-field study was performed in Kenya, where the house-entry response of malaria mosquitoes was recorded. Chapter 5 reports the 3D-analysis of flight tracks by mosquitoes approaching an open eave of an experimental house. Chapter 6 focuses on the responses around this eave in relation to different types of insecticide-treated nets present inside the experimental house.

An applied study on the presence or absence of close-range repellency to insecticidetreated nets is presented in Chapter 7. Mosquito responses and landing behaviours were studied in a wind-tunnel set-up. The methodology links flight behaviour with mosquito health status after exposure to toxic bed nets in combination with host cues.

In the concluding Chapter 8 the main findings are synthesized, and in this chapter I focus on the required research topics for future behavioural studies that adequately support vector-control strategies. 
<smiles>[Y]CCCC</smiles> 


\section{Chapter 2}

\section{Keeping track of mosquitoes: \\ A review of tools to track, record and analyse mosquito flight}

Jeroen Spitzen,

Willem Takken 
The health impact of mosquito-borne diseases causes a huge burden on human societies. Recent vector control campaigns have resulted in promising declines in incidence and prevalence of these diseases, notably malaria, but resistance to insecticides and drugs are on the rise, threatening to overturn these gains. Moreover, several vectorborne diseases have re-emerged, requiring prompt and effective response measures. To improve and properly implement vector control interventions, the behaviour of the vectors must be well understood with detailed examination of mosquito flight being an essential component. Current knowledge on mosquito behaviour across its life history is briefly presented, followed by an overview of recent developments in automated tracking techniques for detailed interpretation of mosquito behaviour. These techniques allow highly accurate recording and observation of mating, feeding and oviposition behaviour. Software programmes built with specific algorithms enable quantification of these behaviours. For example, the crucial role of heat on host landing and the multimodal integration of carbon dioxide $\left(\mathrm{CO}_{2}\right)$ with other host cues, has been unravelled based on three-dimensional tracking of mosquito flight behaviour. Furthermore, the behavioural processes underlying house entry and subsequent host searching and finding can be better understood by analysis of detailed flight recordings. Further potential of these technologies to solve knowledge gaps is discussed. Using tracking techniques can support or replace existing monitoring tools and provide insights on mosquito behaviour that can lead to innovative and more effective vector-control measures.

\section{Abbreviations}

SIT: sterile insect technique

LLINs: long-lasting insecticide-treated nets

IRS: indoor residual spraying

WHO: World Health Organization

DEET: n,n-diethyl-meta-toluamide
2D: two-dimensional

3D: three-dimensional

DLT: direct linear transformation

IR: infrared

fps: frames per second 


\section{Introduction}

Mosquito-borne diseases continue to impose a heavy burden on human societies and impede welfare and economic development (Murray et al., 2012). Despite promising declines of malaria incidence within the last 15 years (WHO-RBM, 2015), other mosquito-borne diseases such as Zika, dengue, chikungunya and West-Nile virus are on the rise and have spread over various continents (Benelli \& Mehlhorn, 2016; Saiz et al., 2016). In addition to climate change, international trade and human transport are considered to be the main drivers of introductions of new vectors, with or without their pathogens, into different geographical regions (Caminade et al., 2014; Imperato, 2016; Kilpatrick, 2011; Medlock \& Leach, 2015; Rezza, 2014).

The recently reported global decline in malaria was achieved mainly by wide-spread use of long-lasting insecticide-treated nets (LLINs), indoor residual spraying (IRS) and proper drug treatment, but insecticide resistance and resistance to antimalarial drugs threaten to prevent further reductions or may even lead to disease resurgence (Benelli et al., 2016; World Health Organization, 2017a). Innovative alternative methods are being developed, such as the use of entomopathogenic fungi (Scholte et al., 2005), biolarvicides (Bacillus spp.) (Boyce et al., 2013; Dambach et al., 2014), push-pull systems and mass trapping techniques using odour-baited traps (Homan et al., 2016; Menger et al., 2015) in combination with house improvements (Tusting et al., 2016). It is expected that the combined use of these tools, along with LLINs, IRS and proper drug treatment, may provide a more sustainable strategy for vectorborne disease control (World Health Organization, 2012). Recently, the World Health Organization launched a strategic approach named Global Vector Control Response, aiming for locally adapted sustainable control measures to target multiple vectors (Alonso et al., 2017; World Health Organization, 2017b).

Successful implementation of new vector control tools along with existing tools following the integrated vector management approach requires detailed understanding of mosquito behaviour. For example, the contact rate of mosquitoes with insecticide-treated bed nets is an important parameter in understanding the efficacy of this technology (Parker et al., 2015; Spitzen et al., 2014); higher efficacy of mosquito traps requires knowledge on trap entry behaviour (Cooperband \& Cardé, 2006; Hiscox et al., 2014; Matowo et al., 2016), and push-pull strategies can be made more effective if mosquito foraging behaviour in the peri-domestic area is well understood (Killeen et al., 2011). 


\section{Chapter 2}

Innovative tracking techniques have opened a new array of possibilities for examining insect behaviour in both the laboratory and (semi-) field (Dickerson et al., 2012; Fry et al., 2000; Kröner et al., 2016). The focus of our review is on the tracking of mosquitoes in space in order to elucidate fundamental aspects of their behaviour in different phases of their life history. An overview of tools used for behavioural tracking of mosquitoes and their technical complexities is provided. We discuss how in-depth knowledge on mosquito behaviour can be exploited for the development and evaluation of vector control strategies.

\section{Behaviour across the mosquito life history}

The mosquito life history traits can be divided into plant feeding, mating, host feeding, oviposition, larval development and pupation. There is surprisingly little fundamental knowledge about the behavioural aspects of these phases, in spite of a wealth of knowledge on the factors affecting these behaviours (Bernáth et al., 2016; Cardé \& Gibson, 2010; Cator et al., 2010; Liu et al., 2010; Liu \& Zwiebel, 2013; Otienoburu et al., 2016; Takken \& Verhulst, 2013). The behavioural aspects of these phases are briefly described below.

We pay attention to anthropophilic mosquito species which forage in and around human dwellings in search of a blood meal (Lyimo \& Ferguson, 2009). For Anopheles species, many of these behaviours occur in the evening or at night, when darkness makes direct observations more challenging. Video-recording makes it possible to visualize mosquito behaviour on a small scale, even during the scotophase, without having the experimental set-up affected by (extra) cues associated with the researcher. Besides host seeking, describing other behaviours during the mosquito life cycle, e.g. mating, (post-) feeding and oviposition, can also greatly benefit the understanding of mosquito-borne disease ecology and further assist in the development and evaluation of surveillance- and intervention tools.

\section{Plant feeding}

Among the first activities of a mosquito after emergence from the pupa is the search for a sugar source. Carbohydrates provide energy for mosquitoes' daily requirements and males rely fully on sugar feeding (Foster, 1995). The sugar source is mostly nectar, but can also consist of fruits, honeydew or extra-floral nectar. Mosquitoes can show strong preferences for certain plant sources (reviewed by Yuval (1992)). Deprivation of sugar sources affects the flight capacity and can consequently affect mosquito dispersal, mating success and/or host-finding (Klowden, 1986; Maïga et al., 2014; Nayar \& van Handel, 1971). 
Recent studies indicate that mosquitoes can learn to associate visual cues with the quality of sugar sources (Bernáth et al., 2016). Such studies contribute to further development of effective use of (toxic) sugar baits to manipulate or control mosquito vectors (Müller et al., 2010; Qualls et al., 2015) or to monitor the presence of pathogens in local populations (Billingsley et al., 1991; Hall-Mendelin et al., 2010). Behavioural studies on sugar feeding can contribute to the knowledge of preferred plant sources, time-budget spent based on efficiency and duration of feeding and possible competition for these sugar sources with other organisms visiting the plant (Foster, 1995; Otienoburu et al., 2016). In addition, questions such as whether mosquitoes are important for pollination can be answered based on behavioural observations; this is a research area showing growing evidence that mosquitoes are not just nectar thieves (Foster, 1995; Inouye, 2010; Peach \& Gries, 2016).

\section{Mating}

Mosquitoes, along with other Diptera, have the special ability to mate in flight (Downes, 1969). Depending on the species, mating occurs in swarms formed by males (most anopheline species) but both sexes can also assemble near emergence sites or around vertebrate hosts (mostly culicine species) (Clements, 1999). Acoustic signals play an important role in mate selection, but the role of pheromones is less clear and only explored for Aedes aegypti (Vanickova et al., 2017). Mating behaviour receives increased attention as a result of the proposed releases of sterile insects (SIT) through irradiation or genetic engineering whereby mate finding and competition are crucial for rapid spread in wild populations (Achinko et al., 2016; Carvalho et al., 2015; Diabate \& Tripet, 2015; Harris et al., 2011; Patil et al., 2015). Furthermore, successful implementation of SIT requires large production of sterile males for which insights in mating behaviour can be used to optimize rearing conditions and boost mosquito colonies (Benelli, 2015a, 2015b). Attempts to study the behaviour of nocturnal males using a camera and infra-red (IR) light date back to 1974, when it was shown that male responsiveness is closely related to their circadian rhythm (Charlwood, 1974). Butail and Manoukis et al. were the first to record, analyse and discern interactions between wild mating swarms of male Anopheles gambiae (Butail et al., 2012; Butail et al., 2013; Manoukis et al., 2009). On a different scale, mating behaviour has been studied in confined areas using tethered mosquitoes (Cator et al., 2010; Potamitis \& Rigakis, 2016; Warren et al., 2009) and follow-up studies in larger arenas with untethered Culex (Simões et al., 2016) and Anopheles mosquitoes (Simões et al., 2017). These behavioural studies provided further evidence how acoustic signals play a role during mate finding and courtship rather than being an epiphenomenon (Gibson et al., 2010). Exploiting the flight tones to which males respond opens a new array of techniques to either enhance or disrupt mating success or to capture 


\section{Chapter 2}

the mosquitoes for monitoring purposes or as a control tool (Balestrino et al., 2016; Benelli, 2015b; Johnson \& Ritchie, 2016; Stone et al., 2013).

\section{Host seeking}

Behavioural research on mosquitoes is dominated by studies on host seeking. This is not surprising, given the direct link with mosquito nuisance and the mosquito capabilities of vectoring human and animal pathogens. Finding a suitable blood host is critical for the uptake of protein needed for vitellogenesis. During host seeking, mosquitoes make use of multiple host-derived cues (Cardé, 2015; Cardé \& Gibson, 2010; McMeniman et al., 2014; Takken \& Knols, 1999) and flight strategies vary among different species (Cardé \& Willis, 2008). Host preference and feeding habits are the main drivers for these different strategies (Takken \& Verhulst, 2013).

Studies on the host-seeking behaviour of mosquitoes focus on the role of specific cues and, ideally, how the integration with other cues drives their orientation. Because of the multi-modal integration, the exact role of a single cue is difficult to determine (McMeniman et al., 2014). Over the years, extensive behavioural studies on the role of $\mathrm{CO}_{2}$, heat, visual cues and specific host odours relevant for host preference have been performed. Outcomes of such studies were often quantified based on responses to a trap and/or by personal observations (Gibson \& Torr, 1999; Gillies, 1974; Gillies \& Wilkes, 1981; Healy \& Copland, 1995; Klun et al., 2013). However, insects display a sequence of behaviours before ending up inside a trap and responses to specific cues that initiate attraction or landing may be missed (Gibson \& Torr, 1999). With the development of digital tracking techniques, more detailed studies can now be performed, allowing links to be made between detailed in-flight behaviours and a combination of host cues (Cardé, 2015; Lacey et al., 2014; McMeniman et al., 2014; Spitzen et al., 2013; van Breugel et al., 2015). The spatial scale at which such studies are performed is strongly correlated with the host cues of interest. The role of heat or the effects of contact repellents, for example, are studied in confined spaces such as small insect cages or wind tunnels (Healy et al., 2002; Lacey \& Cardé, 2011; Menger et al., 2014a; Spitzen et al., 2013; van Breugel et al., 2015). Responses to $\mathrm{CO}_{2}$ in host-seeking activation, source localization and host finding are, on the other hand, studied not only in cages and wind tunnels, but also in (semi-) field settings (Lacey et al., 2014; Lorenz et al., 2013; McMeniman et al., 2014).

We distinguish between fundamental behavioural studies that focus on the host cues (mainly volatiles) that mosquitoes can encounter in the wild, and the cues that may affect these behaviours as a result of interventions. Understanding mosquito 
responses to host cues encountered under natural conditions is an important prerequisite for the correct interpretation of responses to synthetic attractants and/or repellents. Tracking mosquito behaviour to study the effect of (synthetic) attractants, repellents, insecticide-impregnated bed nets or variations in house improvements has shown its relevance in understanding and improving vector-host interventions (Cooperband \& Allan, 2009; Miller \& Gibson, 1994; Parker et al., 2015; Spitzen et al., 2016; Spitzen et al., 2017; Spitzen et al., 2014).

Behavioural resistance to toxicants is relevant for estimating the effectiveness of interventions with LLINs or IRS. Such behavioural effects cannot be measured with standard WHO susceptibility tests, whereas behavioural data obtained with mosquito-tracking tools can be included in predictive models for the effectiveness of control strategies (Briët et al., 2013; Stone et al., 2016).

\section{Oviposition}

Responses to oviposition cues depend on the physiological state of the mosquito (Afify et al., 2014; Qiu et al., 2013; Takken \& Knols, 1999). Female mosquitoes show a temporary absence in behavioural response to host-cues when they are fully engorged with blood (Takken et al., 2001). After maturation of the eggs, oviposition cues take over and responses to host cues are restored after the eggs have been deposited (Davis, 1984; Takken et al., 2001). At the oviposition site, Anopheles and Culex species have been observed to hover above the water and make repeated descents, whereas these behaviours are absent in Ae. aegypti (Kennedy, 1942).

Finding and choosing a suitable breeding site is crucial for gravid female mosquitoes. The water body should retain water long enough for full development of the larvae, contain sufficient nutrients and preferably be free of predators (Suh et al., 2016; Wondwosen et al., 2016). Disrupting this process of oviposition-site selection may be highly beneficial for the implementation of (larval) control methods, either by luring the females to a less-favourable breeding site, e.g. one that is treated with a larvicide, or by luring it into oviposition traps (Fillinger et al., 2003; Kartzinel et al., 2016; Mboera et al., 2000a; Quiroz-Martinez et al., 2012). Efficient lures could also benefit the development of monitoring tools (Eneh et al., 2016). Responses to chemosensory cues that are involved in oviposition-site selection are, however, highly species specific (Afify \& Galizia, 2015). In addition to the chemical components, visual cues also affect site selection (Beehler et al., 1993; Li et al., 2009). The success rate of 'attract-and-kill' methods can benefit from behavioural observations to unravel the range at which (chemical) cues can mediate the desired behaviour (Michaelakis et al., 2007; Schorkopf et al., 2016). Interestingly, oviposition behaviour may also 


\section{Chapter 2}

be affected indirectly, for example following the exposure to spatial repellents at an earlier stage of the mosquito life cycle (Choi et al., 2016).

Behavioural observations on actual egg laying are limited and most studies focus on the 'end result' for choice assays by counting egg distributions in response to certain oviposition cues (Dugassa et al., 2014; Okal et al., 2015; Warburg et al., 2011).

\section{Larval behaviour}

Mosquito larvae are bounded by the breeding site in which they hatched. Their development and survival depend on factors such as water temperature as well as on intra- and inter-species competition over food and space, cannibalism and predation (Blaustein \& Chase, 2007; Grigaltchik et al., 2016; Koenraadt et al., 2004). Behavioural responses of larvae can be triggered by direct physical contact or water movement, fluctuations in light intensity (phototaxis), temperature (thermokinetic) or by chemical stimuli (reviewed by Clements, 1999). Recently, responses to chemical cues by larvae have been studied to further unravel the olfactory signalling pathway of mosquitoes (Liu et al., 2010). 2D tracking of larvae in a Petri dish demonstrated opposite behaviours when exposed to DEET versus yeast. As a follow-up, the thermosensory pathways and larval responses of mosquitoes were studied using a similar setup (Liu \& Zwiebel, 2013).

Behavioural analyses of mosquito larvae are few, but the previously mentioned studies have improved our understanding of sensory response mechanisms in adult mosquitoes (Liu et al., 2010; Liu \& Zwiebel, 2013) and can contribute to model systems for community ecology studies (Blaustein \& Chase, 2007).

\section{Parasite and pathogen mediated mosquito behaviour}

Parasite and/or pathogen infections can affect the energy budget and (flight-) behaviour of mosquitoes and this has consequences for their vector competence (Nyasembe et al., 2014; Scott \& Takken, 2012; Takken et al., 2013). In fact, parasites and pathogens can manipulate mosquito behaviour directly because of the effects of the infection on the mosquito and indirectly through the cues emitted from the infected hosts (Busula et al., 2017; De Moraes et al., 2014; Hurd, 2003; Lacroix et al., 2005; Lefevre \& Thomas, 2008). Working with infected mosquitoes comes with an extra experimental challenge, as the study organisms should not pose a risk to the local environment and their inhabitants. A number of controlled studies demonstrated the role of mosquito infections and how behaviours may be affected to enhance further transmission of the pathogen (Berry et al., 1988; Gleave et al., 2016; Keating et al., 2013; Lee et al., 2000; Newman et al., 2016; Vogels et al., 2017). 
Studies with Plasmodium infections have demonstrated altered feeding behaviours of mosquitoes (Smallegange et al., 2013). Although there is growing evidence that parasite/pathogen-infected mosquitoes express a modified behavioural response, data on actual flight performance of infected mosquitoes are scarce (Cator et al., 2013; Cator et al., 2012; Koella et al., 1998).

Infections with entomopathogenic fungi used as a biopesticide, whether or not in combination with Plasmodium infections, have also demonstrated to have an impact on flight behaviour and host location (Blanford et al., 2005; George et al., 2011; Heinig \& Thomas, 2015; Mnyone et al., 2012; Scholte et al., 2006).

On another level, Wolbachia infections, which have the potential to disrupt pathogen transmission cycles, have been shown to affect mating success, probing behaviour and increased locomotor activities in Ae. aegypti mosquitoes (Chambers et al., 2011; Evans et al., 2009; Moreira et al., 2009; Oliva et al., 2014; Turley et al., 2009).

\section{Insect flight tracking - state of the art}

Image tracking

Mosquito flight recordings were initially processed manually, by describing or quantifying the recorded behaviours (Charlwood, 1974; Healy \& Copland, 1995; Takken \& Knols, 1990). More accurate quantification was conducted by manually digitizing the recorded images: a laborious task, pointing the subject of interest (the mosquito) frame-by-frame. For 3D analysis, the process had to be done twice with a minimum of $2 \times 25$ frames per second (fps), requiring 50 mouse-clicks for one second of video recording (Cooperband \& Cardé, 2006). Machine-vision technology has developed rapidly and with the increase of computer power, development of high-resolution cameras, the possibilities for automated tracking of mosquito behaviour became available (Davies, 2012). In short, the technology starts with acquiring images from cameras which are processed using specific thresholds to identify the object (insect) of interest. The thresholds mainly rely on differences in contrast of the image. Calibration of the set-up is critical, especially if data points from two different cameras will be merged for 3D reconstruction of the flight paths. Generally, the direct linear transformation (DLT) method is used (Woltring, 1980), in which sets of 2D coordinates obtained from the cameras are linked to the known 3D coordinates of markers on a calibration frame. Depending on the lens type used, lens distortion correction is needed. To date, software with codes for automated tracking can be found online as freeware (Brown, 2014; Hedrick, 2008; Hounslow, 2014). Other software packages that cover automated tracking, and sometimes $3 \mathrm{D}$ reconstruction, are mostly custom-made solutions and some have evolved to an add- 


\section{Chapter 2}

on tool next to commercially available motion analysis systems (idTracker, Trackit 3D, Ethovision Track3D) (Fry et al., 2000; Perez-Escudero et al., 2014; Spitzen et al., 2008a). Overall, the technical development has led to a variety of tracking systems to choose from, a reduction in costs and an increase in the portability of recording set-ups. Advantages and disadvantages of quantification techniques have recently been summarized in a non-exhaustive list by Poh et al. (2017). It is remarkable that the table lists a variety of techniques that are described as either 'complex to set up' or 'cover a small area of observation' indicating that there is still much room for improvement. Aspects that need to be taken into account to improve tracking systems are discussed below.

\section{Lighting and contrast}

Successful tracking relies on a clear contrast between the object of interest and the background. The insect is either illuminated against a dark background, or it is depicted against a brighter background. Light conditions of the experimental set-up should not interfere with the natural day-night rhythm of the studied mosquito. For An. gambiae, it has been suggested that filming with infra-red light at $\lambda>900 \mathrm{~nm}$ does not alter the visual environment of the mosquito (Gibson, 1995; Hawkes \& Gibson, 2016). Filming at night requires near-infrared lighting so that the reflection of the light on the mosquito wing is caught by IR-sensitive cameras and is visible against a dark background. A drawback here is that most background materials/ environments also reflect the IR-light, causing unwanted bright areas that make tracking difficult or impossible. The use of black polycarbonate as a background solves this problem (Spitzen et al., 2013). Black fibre glass netting and 'blackboard paint' can serve as alternatives, especially when the contrast of uneven surface areas has to be improved. Another approach is to backlight the cameras so that the mosquito appears as a silhouette in the recorded image (Hawkes \& Gibson, 2016; Parker et al., 2015). Careful evaluation of the experimental requirements is needed to select optimal lighting solutions. This can be extra challenging under (semi-) field conditions.

\section{Multiple object tracking}

From the start of the development of automated tracking techniques, scientists searched for solutions to track multiple objects. Occultations are a challenge and in the case of mosquito flight studies, confined arenas are needed to prevent individuals from flying out of sight, with the risk of being confused with other individuals upon returning to the arena. The best tracking results are currently established with tailormade (MATLAB) tracking codes (Butail et al., 2012; McMeniman et al., 2014; PerezEscudero et al., 2014). Angarita-Jaimes et al. (2016) extended the volume of their 
imaging system by connecting two cameras. This system is able to track multiple mosquitoes within $2.0 \times 1.2 \times 2.0 \mathrm{~m}$, where it should be noted that the presented parameters on mosquito velocity are based on $2 \mathrm{D}$ data. Another way to enlarge the area of interest is by using pan-tilt cameras (Fry et al., 2000; Kurihara et al., 2002). By adding extra cameras, provided that they are properly synchronized, occultations can be reduced and additional accuracy and details of flight tracks can be obtained.

\section{Track the beat}

Another method of 'keeping track' of mosquitoes is by exploiting their wing beats. When the sound that mosquito wings produce is used to record the activity or passage of mosquitoes, we refer to this as acoustic tracking. Literature on responses of mosquitoes to sound is scarce, but dates back to 1949 (Offenhauser Jr \& Kahn, 1949). Acoustic tracking has the potential to be used as a relatively cheap method to monitor behavioural activity of mosquitoes. Sound sensors are generally cheaper than image sensors and there is no additional lighting equipment required. Also, the amount of incoming data is smaller compared to image tracking. Differences in wingbeat frequencies can be used to classify individual mosquitoes to species complex level, however overlap in frequencies does occur (Brogdon, 1994, 1998; Weseka et al., 1998). Field tests report a major challenge in filtering ambient noise (Raman et al., 2007), although a recent study using mobile phones as acoustic sensors managed to filter this noise and mosquito identification was succesful (Mukundarajan et al., 2017). More promising seems the development of opto-acoustic tracking, making use of break-beam technology. When mosquitoes pass a set of infrared emitters and receivers, the interrupted beam is used as an electronic signal by the receiver (Caprio et al., 2001). Species identification using this technique is challenging, but possible by analysing wingbeat patterns and proof of principle was already reported in 1986 (Moore et al., 1986; Ouyang et al., 2015; Potamitis et al., 2015). Automated identification is now reported based on wing movement or wing shape- and pattern analysis and accuracy levels are on the rise (Favret \& Sieracki, 2016; Microsoft, 2015; Pruszynski, 2016; J. Wang et al., 2012; Yang et al., 2015).

\section{Mosquito behaviour at a glance}

Using tracking technology as discussed above, several studies have described the behaviour of mosquitoes during different life stages. We are not aware of studies on oviposition behaviour or plant feeding that have incorporated tracking techniques, but such studies could give additional information on the approach strategies of mosquitoes and measure responses to the provided cues in a (semi-) natural environment (Otienoburu et al., 2016; Suh et al., 2016). Butail et al. (2012) created a $3 \mathrm{D}$ reconstruction of a mating event of wild An. gambiae mosquitoes, a critical step to 


\section{Chapter 2}

characterise the trajectories of both male and female mosquitoes before successful mating occurs. Tracking the behaviour of larvae revealed that thermosensory responses are comparable to those of adult mosquitoes, which may have indirect implications for adult mosquito vectorial capacity, triggered at the larval stage (Liu \& Zwiebel, 2013). The same technique was used to demonstrate that larvae show an altered behaviour after exposure to the mosquito repellent DEET (Liu et al., 2010). In studies on host-seeking female mosquitoes, Spitzen et al. (2013) and McMeniman et al. (2014) described how responses to specific host-cues are integrated and evoke completely different flight patterns, compared to treatments where cues such as heat or $\mathrm{CO}_{2}$ are lacking, or cannot be sensed by mutant mosquitoes. Several studies incorporated mosquito-behavioural analysis in more applied settings directed at interventions with mosquitoes as target. These studies focus on the approach of mosquitoes to host-cues in different settings. The obtained information is used to evaluate the effects of the intervention technique (Cooperband \& Cardé, 2006; Parker et al., 2015), or to provide ideas on how and where to implement interventions (Mnyone et al., 2012; Spitzen et al., 2016). The parameters selected to interpret the behaviours of interest show many similarities across studies. Spatial-temporal distributions reveal the relative attractiveness, and thus importance, of the (host-) cues studied. The change in velocity of the insect is closely related to the distance from- and intensity of- the stimulus and is a measure of orthokinesis. In addition, the velocity parameter is used as an indicator of the insect nearing mechanical barriers, e.g. when mosquitoes approach a house, bed net or host. Change of headings, the intensity of convoluted pathways, sometimes expressed as the number of turns, are closely related parameters and linked to whether mosquitoes are, or have been, tracking odour cues. At close range, locomotor activity or mobility thresholds can be used to determine whether a mosquito is still in flying modus or has landed and is sitting on or near the target. This parameter is also valuable in 2D tracking systems where accurate estimates of flight velocities are lacking. Setting the mobility threshold should be carefully evaluated, as automated tracking can also produce 'movements' while the insect is actually sitting still and the deviation is caused by slight shifts in illuminated pixels (Spitzen et al., 2014). The latter can cause interpretation errors, especially when filming under low resolution.

\section{Data evaluation and considerations before taking off}

The tracking data that are generated need careful evaluation before any meaningful interpretations of the observed behaviours can be made. Occasional tracking errors, for example caused by reflections of IR on interfering objects, should be filtered out to avoid inaccurate data on flight parameters. Such filters can be based on previously-reported maximum flight velocities. Missing data, for example caused by 
lack of resolution or lighting conditions, can be fixed using interpolation. This avoids unnecessary cuts in tracks that would otherwise be appointed to multiple individuals or flight events. Data interpolation seems especially acceptable for set-ups where more than one camera is used, when missing data is only replaced in cases where the other camera 'confirms' the actual continuation of the track (Cooperband \& Cardé, 2006; Spitzen et al., 2013).

Analysing the average value of measured flight parameters over an entire track may not explain the observed behaviour correctly. For example, the flight speed of hostseeking mosquitoes can drastically change when they lose track of an odour or when they come close to their target (Cooperband \& Cardé, 2006; Lacey \& Cardé, 2011; Spitzen et al., 2013). This becomes apparent when the data are divided in sections or bins with certain increments from the target stimuli. With conventional recording speeds ( $25 \mathrm{fps}$ ), however, the number of data points within these smaller sections is limited; this has consequences for the power of the statistical analysis. Recording with higher frame rates solves this problem. For multiple object tracking, high frame rates help to minimize the likelihood of incorrect assignments of individual mosquitoes, as the typical mosquito displacement between frames becomes smaller than the spacing between two individuals (Angarita-Jaimes et al., 2016). High-speed recording, however, comes with other limitations since it requires extra illumination, computer processing speed and data storage.

Filming with high-resolution cameras at high frame rates generates large data files. When recording gigabytes per minute, data storage becomes the limiting factor for the duration of the behavioural experiment. Real-time tracking would be the solution here, where 'only' the $x, y,(z)$ positions of the insect are stored for further analysis and not the video itself. However, the tracking code used should be verifiable using sample videos (Fry et al., 2000; Straw et al., 2011).

\section{Future perspectives}

Integration of spatial observations with fundamental studies on flight dynamics Recent studies on the flight kinematics and aerodynamics of mosquitoes, together with the spatial data reviewed above, throw new light on the way mosquitoes move through their environment (Bomphrey et al., 2017; Dickerson et al., 2012; Muijres et al., 2017). Compared to other similarly-sized insects, mosquitoes fly with exceptionally high wing beat frequencies and low amplitudes (Bomphrey et al., 2017), and after taking a blood meal they can escape from their host without being noticed (Muijres et al., 2017). Although studying the aerodynamics of mosquito flight often requires an experimental set-up that is highly confined in space, it reveals 


\section{Chapter 2}

the physical possibilities and limitations of mosquito flight. Integrating the technical expertise and obtained knowledge on biomechanics with the expanding information on flight behaviours observed in the (semi-) field can accelerate the innovation of vector control tools, for example by fine-tuning fan-powered traps or manipulation of mating behaviour.

\section{How to exploit mosquito behaviour for surveillance and intervention}

With the rapid development and increased availability of tracking hardware and (open-source) software, scientists should consider what system(s) are required to answer their research question(s). This seems obvious, but in the field of mosquito research we distinguish between a focus on fundamental questions on flight behaviour and a more applied approach, where flight data are used for innovation purposes, surveillance, or measuring the effectiveness of intervention strategies. For the applied questions, 2D data are often sufficient and this can drastically reduce the budget required, decrease the amount of incoming raw data and thereby minimize the time required for answering the initial question. For example studies analysing number of landings, or amount of time spent on (insecticide-) treated surfaces, or whether mosquitoes pass certain intervention barriers can benefit from 2D tracking solutions (Cooperband \& Allan, 2009; Sperling et al., 2017; Spitzen et al., 2014). However, when detailed data on the approach of mosquitoes to certain targets, or responses to particular cues is requested, 3D data are a prerequisite because of their typical convoluted flight patterns, both horizontally and vertically (McMeniman et al., 2014; Spitzen et al., 2013).

Our understanding of behavioural repertoires of mosquito vectors during their life cycle, whether infected or not, can be exploited for the development and effective implementation of novel monitoring- and control tools (e.g. Cator et al., 2012; Cook et al., 2007; Homan et al., 2016). Behavioural data can be added to data obtained from conventional monitoring tools such as traps, resting catches and human landing collections, in order to validate models on malaria transmission (Cummins et al., 2012; Killeen et al., 2016). Tracking techniques can be combined with trapping techniques or even replace traps as a surveillance tool to assess mosquito abundance or activity. For the monitoring of species abundance, or measuring the activity of mosquitoes around human dwellings, there is no need to analyse the full behavioural repertoire. The break-beam technology that exploits specific wing beat characteristics, can be advantageous here, especially when combined with a timer to register circadian rhythms (Caprio et al., 2001; Moore et al., 1986; Pruszynski, 2016; Rund et al., 2012). Whereas the use of microphones seems inapplicable for larger arenas because of background noise, the use of opto-acoustics to measure 
activity and classify mosquitoes that enter houses (e.g. via eaves or eave tubes) or traps may provide an easier-to-install and cheaper method than filming. Even if species recognition is imperfect, the technique can still provide data on flight activity on a temporal scale. Given their size, it is unlikely that (harmonic) radar systems will become available to follow long-range movements of mosquitoes (Chapman et al., 2011; Kim et al., 2016; R. Wang et al., 2017). Aerial sampling with balloons at 40$250 \mathrm{~m}$ above ground level revealed that a significant number of mosquitoes exploit wind streams to migrate at high altitudes (Tovi Lehmann, personal communication). Such studies can help to explain seasonal (re-) appearance of mosquito species and predict the need for continuous vector control measures. Mark-recapture studies form another alternative to measure (migration) movements, with the challenge being to capture the wild individual in the first place so that it can be marked (Verhulst et al., 2013). The latter can be solved by using the stable isotope method, with which larvae can be marked and re-captured as adults (Hamer et al., 2012). Markrecapture studies will not reveal details on movements between point $A$ and $B$, but can give valuable information on mosquito dispersal.

\section{Conclusion}

The implementation of vector intervention tools, as part of the Global Vector Control Response strategy launched by the WHO (World Health Organization, 2017b), comes along with questions on the effectiveness and possible behavioural adaptations of mosquitoes to such tools. For example, the shift towards outdoor transmission by behaviourally resistant mosquitoes requires adjustments in intervention techniques (Killeen et al., 2016). Recent developments in insect tracking technology are promising for further implementation in the field and are expected to provide the necessary feedback and estimations of mosquitoes that pass through holes, eaves, windows and doors (Spitzen et al., 2016; Spitzen et al., 2017; Sutcliffe \& Colborn, 2015; Sutcliffe et al., 2017). Interventions may have an effect on airflow coming from houses and quantifying this requires particle tracking of airflows, as reviewed by $\mathrm{Fu}$ et al. (2015). More directly, manipulating the airflow using fog dispensers has shown to affect the flight capabilities of mosquitoes and could function as an intervention tool on itself (Dickerson et al., 2015).

To date, automated tracking techniques have contributed to our understanding of how the multimodal integration of (host-) cues plays a role in source finding of mosquitoes (McMeniman et al., 2014; Spitzen et al., 2013). The tools have provided information on attraction or repellent modes by studying mosquito behaviour around treated surfaces such as bed nets (Lynd \& McCall, 2013; Parker et al., 2015; Parker et al., 2017; Spitzen et al., 2014) or around odour-baited traps (Cooperband \& 


\section{Chapter 2}

Cardé, 2006). Recently, the obtained knowledge led to promising implementations in the field where adding a heat source to an odour-baited trap resulted in a 6.5 fold increase of trap catches of An. gambiae compared to traps without heat (Hawkes et al., 2017).

\section{Acknowledgements}

We thank Florian Muijres, Martin Lankheet, Kees Spoor and colleagues from the Experimental Zoology Group, Wageningen University and Research for discussing and developing automated-tracking solutions. The valuable suggestions offered by Nakul Chitnis of the Swiss Tropical and Public Health Institute and by Tovi Lehmann of the NIAID, NIH, USA are much appreciated. We thank Marcel Dicke for his comments on previous versions of the manuscript. Two anonymous reviewers are acknowledged for their suggestions to improve the manuscript. 
Keeping track of mosquitoes 


$$
8
$$




\section{Chapter 3}

\section{Effect of human odours and positioning of $\mathrm{CO}_{2}$ release point on trap catches of the malaria mosquito Anopheles gambiae sensu stricto in an olfactometer}

Jeroen Spitzen,

Renate C. Smallegange,

Willem Takken 
The anthropophilic malaria mosquito Anopheles gambiae sensu stricto responds to $\mathrm{CO}_{2}$ and human skin emanations. How these odorants affect the behaviour of this mosquito species is studied in an olfactometer. Carbon dioxide is released either as an homogeneous plume or in a turbulent fashion at two different positions from the trap entrance. Anopheles gambiae is deterred from entering a trap with $\mathrm{CO}_{2}$ as the only kairomone, when presented as an homogeneous or turbulent plume. This effect is completely overcome by the addition of skin emanations to the $\mathrm{CO}_{2}$ plume, with a high proportion of mosquitoes found in the trap with skin emanations. Rearrangement of the position of the turbulent $\mathrm{CO}_{2}$ source so that it is $5 \mathrm{~cm}$ downwind of the trap entrance overcomes the deterrent effect of $\mathrm{CO}_{2}$. Carbon dioxide alone, however, does not elicit higher proportions caught compared with clean air. Further studies with the $\mathrm{CO}_{2}$ source positioned $5 \mathrm{~cm}$ downwind of the trap entrance show that skin emanations alone result in fewer mosquitoes entering the trap than $\mathrm{CO}_{2}+$ skin emanations. Skin emanations induce more mosquitoes to fly into a trap than a synthetic blend of $\mathrm{NH}_{3}+$ L-lactic acid when both are combined with $\mathrm{CO}_{2}$. It is concluded that $\mathrm{CO}_{2}$ is a poor kairomone when offered alone and that its presence in the plume at the trap entrance deters mosquitoes from entering. By contrast, when positioned just downwind of the trap entrance, $\mathrm{CO}_{2}$ appears to guide mosquitoes to the vicinity of the trap, where skin emanations then become the principle attractant, causing the mosquito trap entry response. The results of the study have implications for the design of odour-baited traps for this mosquito species. 


\section{Introduction}

Mosquitoes use olfactory cues produced by their hosts to orientate towards them. The essential chemicals that the insects respond to are only partially known and the development of synthetic odours to attract mosquitoes is in its infancy (Costantini et al., 1999; Smallegange et al., 2005; Takken, 1991; Takken \& Knols, 1999). Carbon dioxide appears to be important for all host-seeking mosquito species, but the degree of attractiveness to $\mathrm{CO}_{2}$ varies between species (Mboera \& Takken, 1997). The role of $\mathrm{CO}_{2}$ is assumed to increase with the degree of zoophily (Costantini et al., 1996; Dekker \& Takken, 1998; Dekker et al., 2001; Snow, 1970). For anthropophilic mosquitoes, such as Culex quinquefasciatus and Anopheles gambiae Giles sensu stricto, emanations other than $\mathrm{CO}_{2}$ must play an important role during host seeking to distinguish humans from other $\mathrm{CO}_{2}$ emitting vertebrates. Possibly, the importance of $\mathrm{CO}_{2}$ for anthropophilic mosquitoes increases at greater distances from the host (Sutcliffe, 1987); Takken, 1991). Although, in field studies, the presence of $\mathrm{CO}_{2}$ in a synthetic host-odour mixture increases trap catches of mosquitoes (Costantini et al., 1996; Mboera et al., 2000b; Qiu et al., 2007), windtunnel and bioassay experiments can provide contradicting results. Dekker et al. (2001) suggests that differences reported in the literature about the role of $\mathrm{CO}_{2}$ in host finding by mosquitoes mainly arise because of differences in plume structure and trapping methods. In laboratory studies, Dekker et al. (2001) show that An. gambiae s.s. females respond with an upwind flight to a source of homogeneous $\mathrm{CO}_{2}$, but do not enter the trap. However, when using a turbulent $\mathrm{CO}_{2}$ plume, mosquitoes do enter the trap but no more than in the control with clean air. These findings are further corroborated by work on Aedes aegypti, Cx. quinquefasciatus and Cx. tarsalis (Cooperband \& Cardé, 2006; Dekker et al., 2005).

In the present paper, the interaction of $\mathrm{CO}_{2}$ with human-specific odours is studied. For haematophagous arthropods $\mathrm{CO}_{2}$ is a common kairomone signalling the presence of a vertebrate. However, for an anthropophilic mosquito, other host-specific cues are expected to play a crucial role in host location. Consequently, it is anticipated that there is a change in response to $\mathrm{CO}_{2}$ during the host-location process. As a result of this shift, discrimination for human-specific odours is expected to take place. The effects of $\mathrm{CO}_{2}$ are studied when combined with natural human odours or synthetic odour blends on the behaviour of An. gambiae s.s. (hence termed An. gambiae) in a dual-choice olfactometer. Various methods of releasing $\mathrm{CO}_{2}$ are tested. In addition to the method used by Dekker et al. (2001), the $\mathrm{CO}_{2}$ source is not only situated in the trap, but also placed downwind of the trap entrance, because electric nets in front of the trap entrance demonstrate that $\mathrm{CO}_{2}$ guides An. gambiae towards the trap but this does not always result in higher trap entry (Dekker et al., 2001). Differences in 


\section{Chapter 3}

response as a result of homogeneous and turbulent odour plumes and the position of the $\mathrm{CO}_{2}$ source are discussed with relevance for host orientation and trap design.

\section{Materials and methods}

\section{Mosquitoes}

The An. gambiae colony at Wageningen University, The Netherlands, originated from Liberia in 1987 and has been reared on blood from a human arm from 1988 onward. It was maintained under an LD 12 : $12 \mathrm{~h}$ scoto-photophase at $27 \pm 1{ }^{\circ} \mathrm{C}$ and $80 \pm 5 \%$ $\mathrm{RH}$. Adults were kept in gauze cages $(30 \times 30 \times 30 \mathrm{~cm})$ and were provided with a $6 \%$ glucose solution ad libitum and offered a blood meal twice weekly for $10 \mathrm{~min}$. Eggs were laid on wet filter paper and transferred to water trays before hatching. Larvae were fed Tetramin (Tetrawerke, Germany) fish food daily. Pupae were removed from the trays daily and allowed to emerge inside the cage used for keeping adults.

Teneral female mosquitoes, 5-7 days-old, were transferred to release cages 14-16 $\mathrm{h}$ before testing. Each release cage contained 30 mosquitoes and was provided with a water-soaked cotton wick to prevent desiccation. For each bio-assay, new mosquitoes were used.

\section{Olfactometer experiments}

The response of An. gambiae females to different structures of odour plume was examined in a dual-choice olfactometer $(1.6 \times 0.6 \times 0.6 \mathrm{~m})$ modified after (Pates et al., 2001) (Figure 1). Three different $\mathrm{CO}_{2}$ plumes were used: homogeneous and turbulent $\mathrm{CO}_{2}$ plumes, created by releasing $\mathrm{CO}_{2}$ within the trap as in Dekker et al. (2001), and thirdly, a turbulent plume was created inside the flight chamber and placed $5 \mathrm{~cm}$ downwind from the trap entrance (Figure 1CIII). The concentration of $\mathrm{CO}_{2}$ inside the olfactometer was measured at three different points (Figure 1A,B) using a Xentra $4100 \mathrm{CO}_{2}$ analyser (Servomex, The Netherlands) at intervals of 3 min. From the second series of experiments onwards, two $\mathrm{CO}_{2}$ measuring points were placed in the middle of the tunnel. One point was in line with the right trap entrance and the other point in line with the left trap entrance. This rearrangement of $\mathrm{CO}_{2}$ measuring points was made because of the introduction of $\mathrm{CO}_{2}$ downwind from the trap entrance. The analyser was programmed to shift from one point to the other every min, with 60 successive readings per min. Carbon dioxide concentrations were measured over a range of $0-1030 \mathrm{ppm}$ with an accuracy of $0.1 \mathrm{ppm}$. The data were downloaded to a PC using Das Wizard 2.0 software (Measurement Computing Corporation, Norton, MA).

For indications of $\mathrm{CO}_{2}$ concentrations in the flight chamber above the range of the analyser (> $1030 \mathrm{ppm}$ ) calculations can be made to obtain an impression of the real 
concentration, taking the following factors into account. The $\mathrm{CO}_{2}$, at a concentration of $4.5 \%$, was released from a sample bag with a constant air flow of either $250 \mathrm{~mL}$ (turbulent plume) or $2 \mathrm{~L}$ (homogeneous plume) per min and mixed with clean air inside the trapping device. This air stream, with a regulated speed of $20 \mathrm{~cm} \mathrm{~s}$, left the trap via the trap entrance (diameter $5 \mathrm{~cm}$ ) towards the flight chamber with a cross-sectional surface area of $3600 \mathrm{~cm}^{2}$. Once in the chamber, the airflow drops rapidly towards the minimum measurable value of $1 \mathrm{~cm} \mathrm{~s}^{-1}$ (at $30 \mathrm{~cm}$ from the trap entrance). Two examples are given with the average concentration of $521 \mathrm{ppm} \mathrm{CO}_{2}$ found for clean air during the first series of experiments.

Example 1: $\mathrm{CO}_{2}$ concentration of a homogeneous plume at the trap entrance. Crosssectional surface area of cylinder (trap entrance): $\pi r^{2}=\pi 6.25=19.63 \mathrm{~cm}^{2}$. Velocity of air stream at the trap entrance is: $20 \mathrm{~cm} \mathrm{~s}^{-1}=1200 \mathrm{~cm} \mathrm{min.} .^{-1}$. This air stream is a combination of $2 \mathrm{~L} \mathrm{~min}^{-1}, 4.5 \% \mathrm{CO}_{2}=2000 \mathrm{~cm}^{3} / 19.63 \mathrm{~cm}^{2}=101.9 \mathrm{~cm} \mathrm{~min}^{-1}$ and $1200-101.9=1098.1 \mathrm{~cm} \mathrm{~min}^{-1}$ clean moist air with a $\mathrm{CO}_{2}$ concentration of $521 \mathrm{ppm}$.
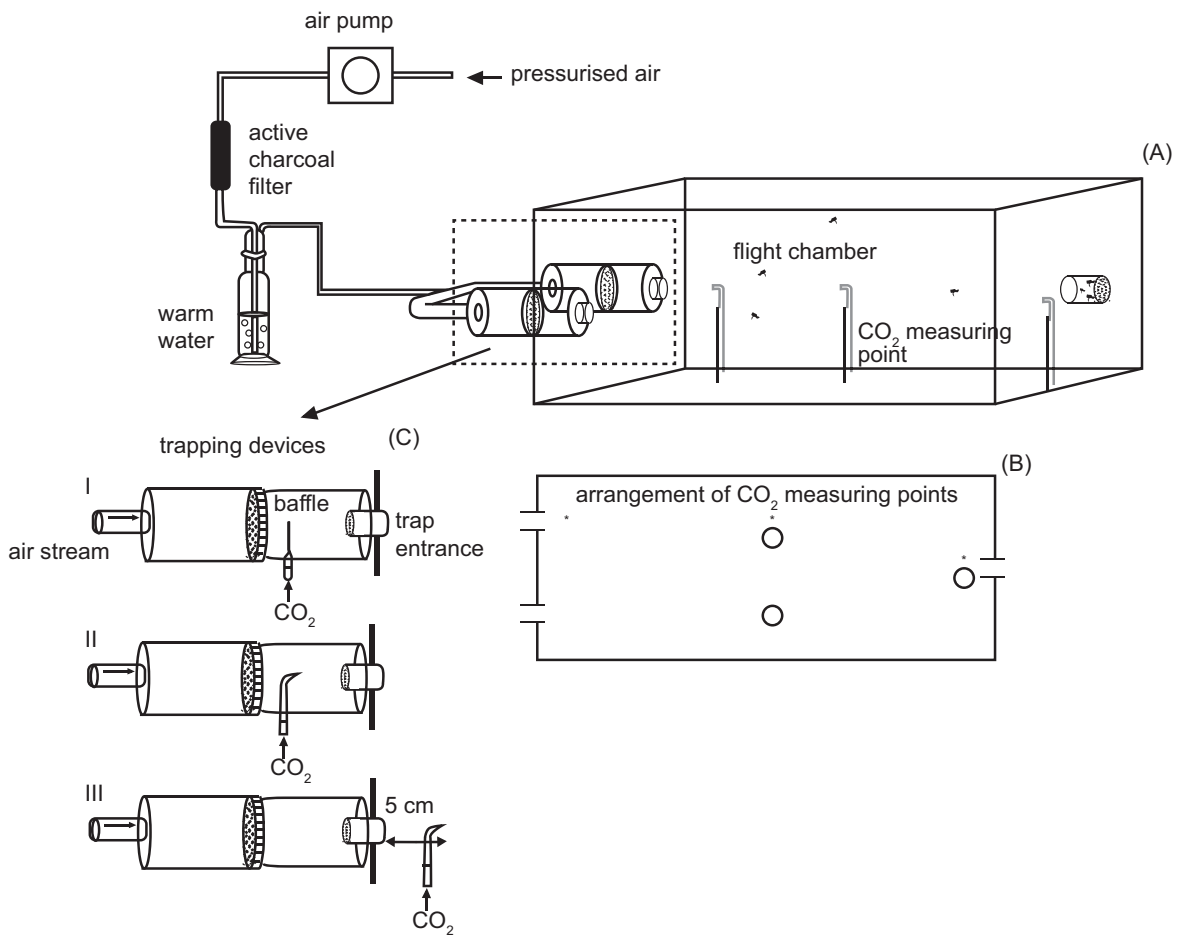

Figure 1. (A) Diagram of the dual-choice olfactometer (modified after Pates et al., 2001) and the three $\mathrm{CO}_{2}$ measuring points. (B) Shows a top view from the chamber and the rearrangement of the $\mathrm{CO}_{2}$ measuring points during the second and third series of experiments. * Position during the first series, $\circ$ Position during the second and third series. (C) Represents a trapping device and the three different tested release methods of $\mathrm{CO}_{2}$. I and II show the position of the pipettes used to create an homogeneous and a turbulent $\mathrm{CO}_{2}$ plume, respectively (after Dekker et al., 2001). III shows the position of the $\mathrm{CO}_{2}$ source that was placed downwind of the trap entrance. 


\section{Chapter 3}

For this example, the concentration of $\mathrm{CO}_{2}$ in the air stream at the trap entrance is estimated to be: $(101.9 \times 45000)+(1098.1 \times 521) / 1200=4297 \mathrm{ppm}$.

Example 2: $\mathrm{CO}_{2}$ concentration of a homogeneous plume in the middle of the flight chamber. Cross-sectional surface area of flight chamber: $\mathrm{h} \times \mathrm{w}=60 \mathrm{~cm} \times 60 \mathrm{~cm}=$ $3600 \mathrm{~cm}^{2}$. Velocity of air stream in the middle of the chamber: approximately $1 \mathrm{~cm}$ $\mathrm{s}^{-1}=60 \mathrm{~cm} \mathrm{~min}^{-1}$. This air stream is a combination of $2 \mathrm{~L} \mathrm{~min}^{-1}, 4.5 \% \mathrm{CO}_{2}=2000$ $\mathrm{cm}^{3} / 3600 \mathrm{~cm}^{2}=0.56 \mathrm{~cm} \mathrm{~min}^{-1}$ and $60-0.56=59.44 \mathrm{~cm} \mathrm{~min}^{-1}$ clean moist air with a $\mathrm{CO}_{2}$ concentration of $521 \mathrm{ppm}$. The concentration of $\mathrm{CO}_{2}$ in the middle of the chamber is estimated as: $(0.56 \times 45000)+(59.44 \times 521) / 60=932.8 \mathrm{ppm}$. The same calculations can be made for a turbulent plume keeping in mind that the flow of $\mathrm{CO}_{2}$ is eight-fold lower.

Thirty female An. gambiae were released $160 \mathrm{~cm}$ downwind from the trap openings $(5 \mathrm{~cm}$ in diameter, $30 \mathrm{~cm}$ apart) from which two air streams (mean $=21.3 \pm 1.5$ $\mathrm{cm} \mathrm{s}^{-1}$ ) flowed. After $15 \mathrm{~min}$, mosquitoes that were caught in the trapping devices were counted and the trapping devices replaced with clean ones. Uncaught mosquitoes were removed from the olfactometer before a new group was released. All experiments took place during the last $4 \mathrm{~h}$ of the scotophase, when An. gambiae females are known to be responsive to host odours (Haddow \& Ssenkubuge, 1973; Maxwell et al., 1998; Killeen et al., 2006).

The stimuli used were $4.5 \% \mathrm{CO}_{2}$ released from a $80 \mathrm{~L}$ Tedlar gas sample bag (SKC Inc., Eighty Four, PA) either as an homogeneous plume (2 $\left.\mathrm{L} \mathrm{min}^{-1}\right)$ inside the trap, a turbulent plume $\left(230 \mathrm{~mL} \mathrm{~min}^{-1}\right)$ inside the trap (Dekker et al., 2001) or a turbulent plume (230 mL min.-1) placed $5 \mathrm{~cm}$ downwind of the trap opening. The stimuli were tested against or in combination with: clean moist air (CMA), a nylon stocking worn for $24 \mathrm{~h}$ by J.S. (sock) or a blend of ammonia and lactic acid $\left(\mathrm{NH}_{3}+\right.$ L-lactic acid). This $\mathrm{NH}_{3}$-La mixture was obtained by combining air from a $2.5 \%$ ammonia sampling bag (136 ppm, 230mL min-1; Smallegange et al., 2005) with charcoal-filtered air (15 $\mathrm{mL} \mathrm{min}^{-1}$ ) that passed through a 250-mL Erlenmeyer flask (Fisher Scientific BV, The Netherlands) filled with a $90 \%$ aqueous L-lactic acid solution (analytical grade, Purac Bioquimica, Spain).

Three series of experiments were conducted. The tested treatments were CMA, homogeneous $\mathrm{CO}_{2}(\mathrm{hC})$, turbulent $\mathrm{CO}_{2}(\mathrm{tC})$, turbulent $\mathrm{CO}_{2}$ downwind ( $\mathrm{tCdw}$ ), worn nylon sock ( $\mathrm{Sk}$ ), ammonia $\left(\mathrm{NH}_{3}\right)$ and lactic acid (La). Each treatment was replicated five times and the sequence of treatments was randomized within each test day and between days. Test stimuli were alternated between the right and left ports in different 
replicates to rule out positional effects. Clean moist air from both ports was tested on each test day to verify symmetry of the olfactometer. During these symmetry tests, $\mathrm{CO}_{2}$ background levels at different points within the flight chamber were measured.

\section{Statistical analysis}

The ratio of the number of mosquitoes that were caught in both traps over the number that had flown out of the release cage during the experimental time (15 $\mathrm{min}$ ) was considered as the trap entry response. Potential differences in response for all experimental series were estimated using a Generalized Linear Model (Genstat, release 8.11; VSN International, UK). Differences between treatment and control were tested by comparing the total number of mosquitoes caught in both traps using a chi-square test.

\section{Results}

Trap catches

A total of 2478 mosquitoes was tested, of which 1532 (61.8\%) flew into one of the upwind traps. The experiments with clean air in both ports demonstrated that the olfactometer was symmetrical $(P=0.486)$. The first series of experiments showed that both homogeneous and turbulent $\mathrm{CO}_{2}$ plumes caused fewer mosquitoes to enter the trap than clean moist air when tested against each other $(P<0.001$ and $P=0.011$, respectively; Figure $2 \mathrm{~A}$ ). When a worn sock was placed in both traps, the effect of $\mathrm{CO}_{2}$ was negligible and the trap entry response was significantly higher compared with the experiment in which homogeneous $\mathrm{CO}_{2}$ was the only kairomone $(P=0.008)$. Although trap catches of skin emanations $+\mathrm{CO}_{2}$ (homogeneous or turbulent) were also greater than those with turbulent $\mathrm{CO}_{2}$ alone, the difference in response was not significant $(P=0.170$ and $P=0.131$, respectively).

When homogeneous and turbulent $\mathrm{CO}_{2}$ in combination with skin emanations were tested against each other, the difference in trap entries was not different $(P=0.100)$, and the trap entry response was high (Figure $2 \mathrm{~B}$ ). When the $\mathrm{CO}_{2}$ source was placed just downwind from the trap entrance, trap catches were not reduced $(P=0.339)$ in contrast to $\mathrm{CO}_{2}$ released from the trap entrance, and the trap entry response was similar $(P>0.05)$ to that in the presence of skin emanations (Figure 2B). Homogeneous $\mathrm{CO}_{2}+$ skin emanations, released from the trap entrance caused the same trap entry response as turbulent $\mathrm{CO}_{2}$ placed downwind from the trap entrance and provided with skin odour $(P=1.000)$. However, when the 'downwind' position of the turbulent $\mathrm{CO}_{2}$ plume was tested against the 'inside trap' position and a sock was placed in both traps, more mosquitoes were caught with the 'downwind' $\mathrm{CO}_{2}$ release method $(P=0.004)$. The combination of turbulent $\mathrm{CO}_{2}$ placed 'downwind' + 


\section{Chapter 3}

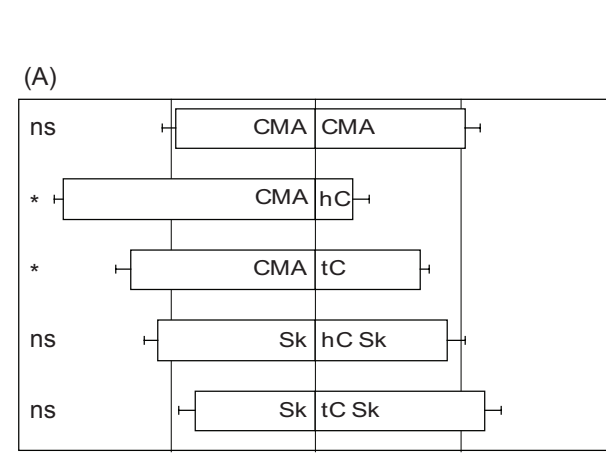

Total

response (\%)

24.9 a

$35.8 \mathrm{ab}$

$60.4 \mathrm{ad}$

$83.8 \mathrm{~cd}$

$81.8 \mathrm{~cd}$
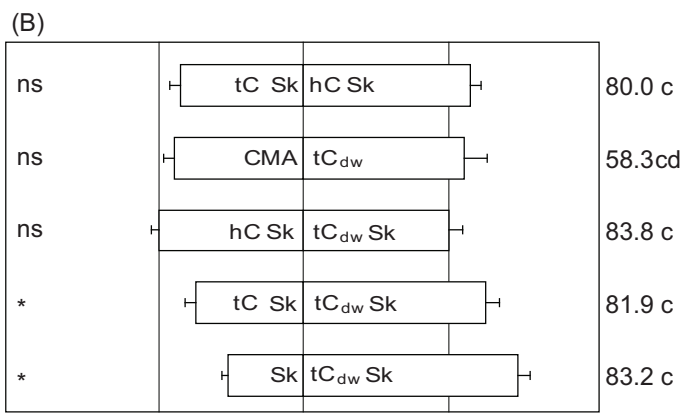

(C)

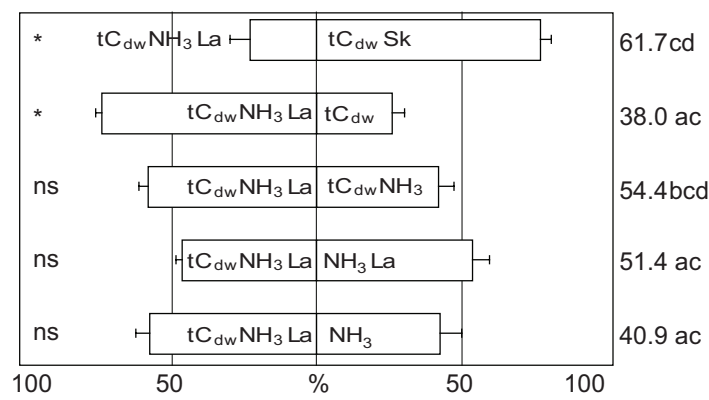

Figure 2. Mean responses of Anopheles gambiae to homogeneous $\mathrm{CO}_{2}$ plumes (hC), turbulent $\mathrm{CO}_{2}$ plumes ( $\mathrm{tC}$ ) or turbulent $\mathrm{CO}_{2}$ plumes placed downwind of the trap entrance $\left(\mathrm{tC}_{\mathrm{dw}}\right)$ tested in combination with or against clean moist air (CMA), worn sock (Sk), ammonia $\left(\mathrm{NH}_{3}\right)$ or lactic acid (La). Chi-square test: ${ }^{*} P<0.001$, ns, not significant. The total percentages of mosquitoes flying into either trap are shown at the right: two-choice tests marked with different letters resulted in significantly different trap entry responses (Generalized Linear Model).

skin odour caught significantly $(P<0.001)$ more mosquitoes than skin odour alone. In all cases of the second series of experiments (Figure 2B), trap entry responses were not different from each other but significantly higher $(P<0.05)$ than the CMAonly treatment. 
Mosquitoes chose significantly more often the combination of turbulent $\mathrm{CO}_{2 \mathrm{dw}}+$ skin emanations than turbulent $\mathrm{CO}_{2 \mathrm{dw}}+\mathrm{NH}_{3}+\mathrm{L}$-lactic acid $(P<0.001$, Figure $2 \mathrm{C})$. However, the synthetic mixture + turbulent $\mathrm{CO}_{2 \mathrm{dw}}$ was more attractive than turbulent $\mathrm{CO}_{2 \mathrm{dw}}(P<0.001)$ but not different from the synthetic mixture alone $(P=0.491)$. Catches with $\mathrm{CO}_{2 \mathrm{dw}}+\mathrm{NH}_{3}$ were not different from that of $\mathrm{CO}_{2 \mathrm{dw}}+\mathrm{NH}_{3}+\mathrm{L}$-lactic acid $(P=0.148) . \mathrm{NH}_{3}+\mathrm{L}$-lactic acid and $\mathrm{NH}_{3}$ alone were as attractive to the mosquitoes as $\mathrm{CO}_{2 \mathrm{dw}}+\mathrm{NH}_{3}+\mathrm{L}$-lactic acid $(P=0.491$ and $P=0.249$, respectively, Figure $2 \mathrm{C})$. Trap entry responses with $\mathrm{NH}_{3}+\mathrm{L}$-lactic acid $+\mathrm{CO}_{2}$ (Figure $2 \mathrm{C}$ ) were not significantly different from that of skin emanations $+\mathrm{CO}_{2}$ (Figure 2A, B) $(P>0.05)$.

\section{Carbon dioxide concentrations}

To ensure symmetry, background $\mathrm{CO}_{2}$ levels were calculated from all $\mathrm{CO}_{2}$ readings measured on each day during the tests. The mean $\pm \mathrm{SD} \mathrm{CO}_{2}$ level in the flight chamber during these tests was $514 \pm 65 \mathrm{ppm}$. The $\mathrm{CO}_{2}$ concentration measured in front of a trap with clean moist air was $521 \pm 87 \mathrm{ppm}$.

When turbulent or homogeneous plumes were measured in front of the trap, $\mathrm{CO}_{2}$ levels exceeded the maximum settings of the analyser with a level of $1030 \mathrm{ppm}$ (see Materials and Methods). As soon as an homogeneous $\mathrm{CO}_{2}$ plume was released from one of the traps, the entire olfactometer was filled with $\mathrm{CO}_{2}$ levels above 1030 ppm after approximately $6 \mathrm{~min}$ (Figure 3A). This was not the case when $\mathrm{CO}_{2}$ was released as a turbulent plume: the levels of $\mathrm{CO}_{2}$ measured at median and downwind sections of the flight chamber were then in the range 550-650 ppm (Figure 3B).

In the second and third series of experiments, the $\mathrm{CO}_{2}$ concentrations were only measured at mid flight chamber and in front of the release cage of the mosquitoes. When releasing a turbulent $\mathrm{CO}_{2}$ plume $5 \mathrm{~cm}$ downwind of one of the trap entrances, $\mathrm{CO}_{2}$ concentrations in the mid section were approximately $50 \mathrm{ppm}$ higher on this side of the tunnel than on the other side, from which no $\mathrm{CO}_{2}$ was released (Figure $3 \mathrm{C}$ ). As soon as an homogeneous plume was released from one of the traps, the olfactometer was filled with $\mathrm{CO}_{2}$ levels above $1030 \mathrm{ppm}$, as during the first series of experiments (Figure 3A).

\section{Discussion}

The findings show that $\mathrm{CO}_{2}$ and human skin emanations affect the behaviour of $A n$. gambiae females in various ways: skin emanations are attractive, but in combination with $\mathrm{CO}_{2}$, the attraction is significantly enhanced. Carbon dioxide however, is a kairomone on its own, but acts as a deterrent at close range, as shown by the reduced trap catch. These findings correspond with the results of previous studies by Dekker et 


\section{Chapter 3}

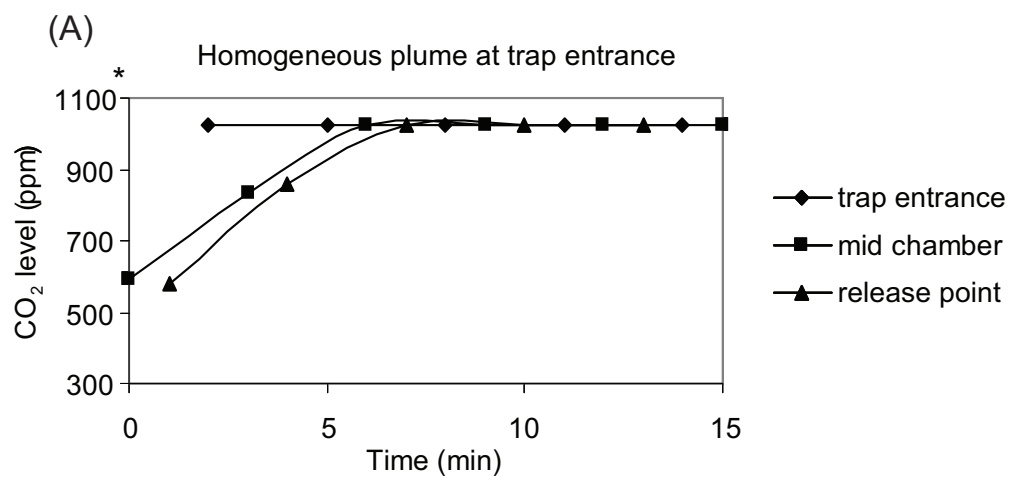

(B)

Turbulent plume at trap entrance

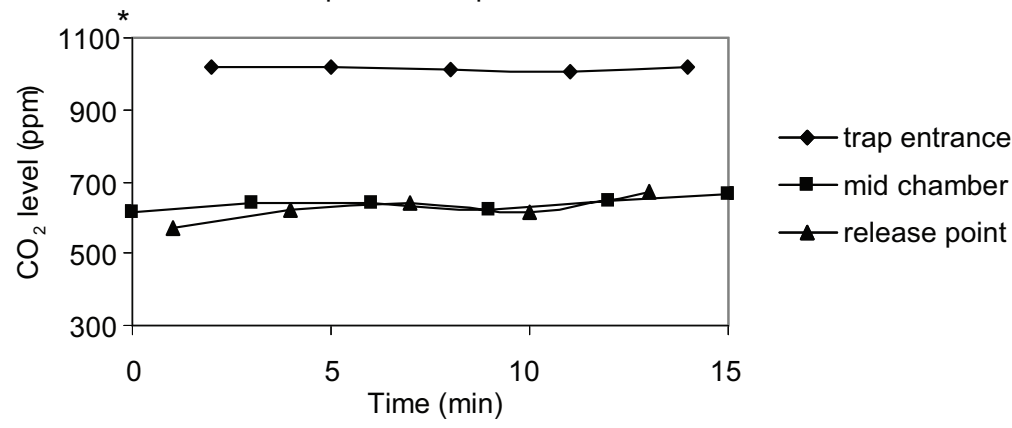

(C)

Turbulent plume position $5 \mathrm{~cm}$ downwind

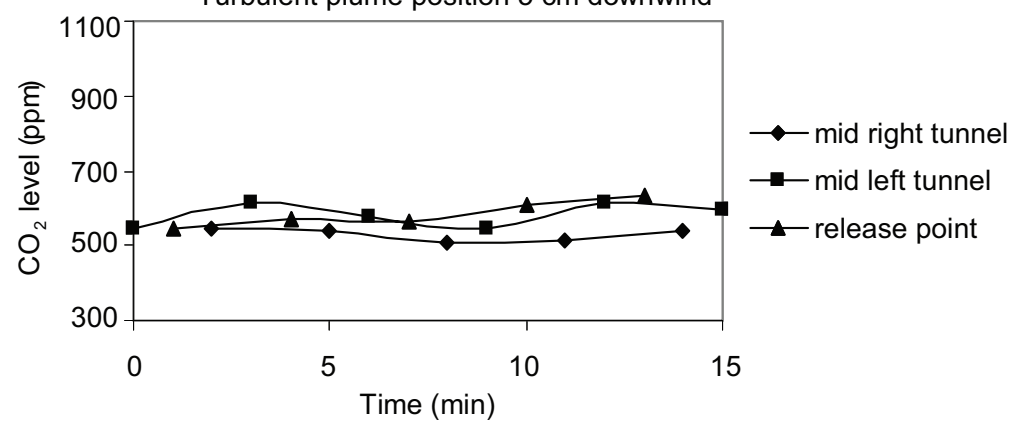

Figure 3. (A) Carbon dioxide levels (ppm) measured at three different points in the flight chamber when releasing an homogeneous $\mathrm{CO}_{2}$ plume from the trap entrance. ${ }^{*} \mathrm{CO}_{2}$ analyser could not measure $\mathrm{CO}_{2}$ concentrations above 1030 ppm (see calculations in Materials and Methods). (B) Carbon dioxide levels (ppm) measured at three different points in the flight chamber when releasing a turbulent $\mathrm{CO}_{2}$ plume from the trap entrance. ${ }^{*} \mathrm{CO}_{2}$ analyser could not measure $\mathrm{CO}_{2}$ concentrations above $1030 \mathrm{ppm}$ (see calculations in Materials and Methods). (C) Carbon dioxide levels (ppm) measured at three different points in the flight chamber when releasing a turbulent $\mathrm{CO}_{2}$ plume downwind from the trap entrance. The $\mathrm{CO}_{2}$ measuring point 'mid left tunnel' is in line with the $\mathrm{CO}_{2}$ source, which was placed downwind of the left trap entrance.

al. (2001), using the same olfactometer. Releasing $\mathrm{CO}_{2}$ from within the trap causes an inhibition of the mosquito catch with homogeneous and turbulent $\mathrm{CO}_{2}$ plumes. Addition of human skin odour to the $\mathrm{CO}_{2}$ plume overrules the inhibitive effect of $\mathrm{CO}_{2}$ irrespective 
of the physical composition of the $\mathrm{CO}_{2}$ plume. This appears to contradict the results of Dekker et al. (2001), but can be explained by their findings of a dose-dependent inhibition effect for increased concentrations of $\mathrm{CO}_{2}$. The deterrent effects are only apparent in terms of attraction, considering the number of mosquitoes that are found in the trap, but not in terms of activation and orientation. This becomes clear from the present results during the second series of experiments, when a $\mathrm{CO}_{2}$ plume is created downwind of the trap entrance. In this case, the deterrent effect of $\mathrm{CO}_{2}$, expressed as number of mosquitoes found in a trap, is no longer present, and in the presence of human skin odour, more mosquitoes are caught in the trap upwind of the $\mathrm{CO}_{2 \mathrm{dw}}$ source than in the trap with skin odours only. This result appears to be caused by mosquitoes flying upwind in the turbulent $\mathrm{CO}_{2}$ plume and near the $\mathrm{CO}_{2}$ source, being guided by the skin odours passing over the source. Dekker et al. (2001) demonstrated this by placing an electrocuting grid just downwind from the traps. The role of $\mathrm{CO}_{2}$ and the difference between attraction and activation is discussed by Gillies (1980) and Takken (1991). Both authors define $\mathrm{CO}_{2}$ as an activating compound, but also suggest kairomone properties. During the activation process, mosquitoes fly upwind without the ability to orientate to the source (Takken et al., 1997). Hence, in the present study, $\mathrm{CO}_{2}$ acts to bring mosquitoes to the vicinity of the trap as demonstrated by the hypothetical flight paths shown in Figure 4. In Figure 4(A), a mosquito is exposed to an homogeneous plume of $\mathrm{CO}_{2}$, which prevents it from entering the trap whereas another mosquito flying outside the $\mathrm{CO}_{2}$ plume readily enters the trap without $\mathrm{CO}_{2}$; in Figure 4(B), a mosquito is exposed to turbulent $\mathrm{CO}_{2}$, which acts as a stimulus to lead it to the vicinity of the odour source, but agitated flight behaviour caused by $\mathrm{CO}_{2}$ prevents trap entry. The mosquito, however, may enter the other trap; in Figure 4(C), a source of turbulent $\mathrm{CO}_{2}$ placed $5 \mathrm{~cm}$ in front of the trap entrance acts to bring the mosquito to the vicinity of the trap entrance. Trap entry may follow as in the other trap without $\mathrm{CO}_{2}$. Dekker et al. (2001) suggest that, under $\mathrm{CO}_{2}$ stimulation, the trap entrance is too narrow for the mosquitoes to continue upwind flight. Skin odours, however, overrule this effect and allow the mosquitoes to orientate to the human odours and guide them to the source. The current data show that mosquitoes enter traps releasing human odours and that the human odour suppresses the activating effect caused by $\mathrm{CO}_{2}$, allowing the mosquitoes to navigate the relatively narrow trap entrance. The release of $\mathrm{CO}_{2}$ immediately downwind from the ports results in a significantly greater catch with $\mathrm{CO}_{2}$ + skin odours compared with skin odours alone. This suggests that mosquitoes are activated by the $\mathrm{CO}_{2}$ and then, at close range, followed the plume of skin emanations leading them into the trap. These findings are in line with Takkens (1991) suggestion that $\mathrm{CO}_{2}$ acts from a distance whereas skin emanations may be considered shortrange attractants. The exact location of $\mathrm{CO}_{2}$ release is important for the development of efficient mosquito traps. The approach towards traps differs between trap type and 


\section{Chapter 3}
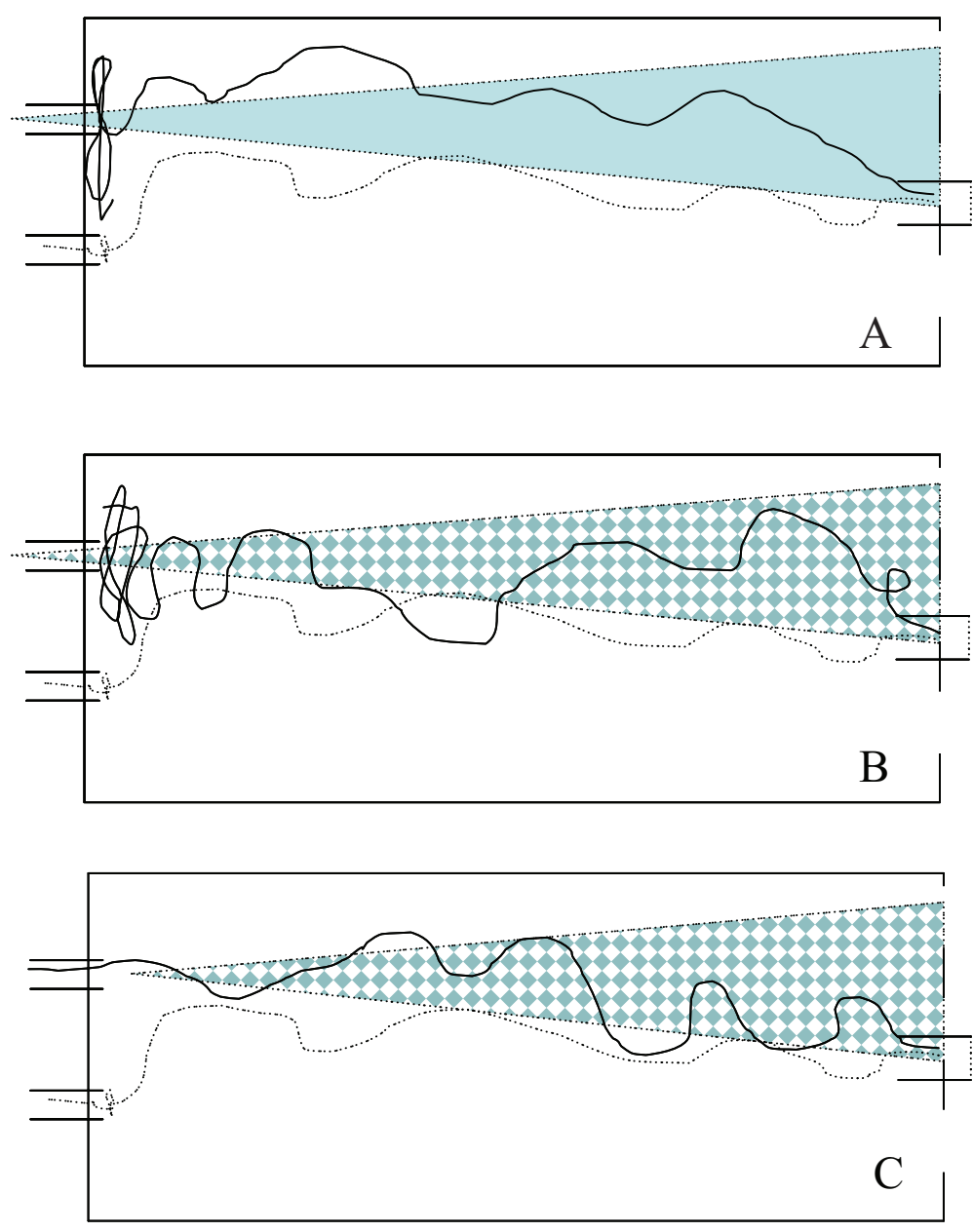

Figure 4. The three $\mathrm{CO}_{2}$ plumes in the olfactometer and hypothetical upwind flight paths. (A) homogeneous $\mathrm{CO}_{2}$ released from trap entrance; (B) turbulent $\mathrm{CO}_{2}$ released from trap entrance; (C) turbulent $\mathrm{CO}_{2}$ released $5 \mathrm{~cm}$ downwind of the trap entrance. Mosquitoes are avoiding the traps if $\mathrm{CO}_{2}$ is released from within the traps. For test conditions, see Figure 2: (A) clean moist air (CMA) versus homogeneous $\mathrm{CO}_{2}$ plumes; (B) CMA versus turbulent $\mathrm{CO}_{2}$ plumes; (C) $\mathrm{CMA}$ versus turbulent $\mathrm{CO}_{2}$ plumes placed downwind of the trap entrance.

mosquito species as demonstrated during a semi-field experiment with $C x$. tarsalis and Cx. quinquefasciatus on trap efficiency (Cooperband \& Cardé, 2006).

Previously, the attractive effect of $\mathrm{NH}_{3}+$ L-lactic acid for An. gambiae has been reported (Smallegange et al., 2005). In the present study, it is demonstrated that the blend of $\mathrm{NH}_{3}$ + lactic acid alone is less attractive compared with natural skin odours, but, when combined with turbulent $\mathrm{CO}_{2}$ released downwind from the trap entrance, more mosquitoes are caught with this blend compared with $\mathrm{CO}_{2}$ alone. These results also show that essential kairomones are lacking in the synthetic mixture because 
natural skin odours $+\mathrm{CO}_{2}$ are found to be significantly more attractive.

Although $\mathrm{CO}_{2}$ is a strong kairomone for many mosquito species (Mboera \& Takken, $1997)$, its role in An. gambiae is less clear. Differences in $\mathrm{CO}_{2}$ levels between background (514 ppm) and odour plume (550-1030 ppm) are sufficiently high to activate $\mathrm{CO}_{2}$ receptors (Grant et al., 1995). As long as small differences in $\mathrm{CO}_{2}$ concentrations in filaments and background are present, the insects receive sufficient stimulation for continued upwind flight (Dekker et al., 2005). The background concentration of $\mathrm{CO}_{2}$ (approximately $500 \mathrm{ppm}$ ) appears to be high compared with the concentration in the atmosphere (approximately 370 ppm; Zhou, et al., (2006) possibly because of working indoors. Stange (1997) shows that an increased background concentration reduces host-signal detection ability within (herbivorous) moths. However, Grant et al. (1995) report, from electrophysiological experiments, that $\mathrm{Ae}$. aegypti is little influenced by the background concentration of $\mathrm{CO}_{2}$ and that the mosquito is able to respond to step increases of $50 \mathrm{ppm}$. Furthermore, $A e$. aegypti neurones have a sufficient response range to cover the whole span of $\mathrm{CO}_{2}$ concentrations likely to be encountered during host seeking (0-4000 ppm). Similar sensitivity is seen for Anopheles stephensi and also for An. gambiae (A. Grant, personal communication cited in Takken \& Knols, 1999; Qiu et al., 2007).

The highest trap catches are obtained when releasing human skin odours, corresponding with previous work where An. gambiae responds strongly to human skin odours (Haddow, 1942; de Jong \& Knols, 1995; Qiu et al., 2006). Carbon dioxide contributes up to $50 \%$ of all An. gambiae collected in traps when human odour $+\mathrm{CO}_{2}$ is released in field studies in Burkina Faso (Costantini et al., 1996). In other studies, the role of $\mathrm{CO}_{2}$ in the overall attraction of $A n$. gambiae is negligible (Mboera \& Takken, 1997). In a recent study in the Gambia, $\mathrm{CO}_{2}$ is found to be an essential kairomone for the collection of An. gambiae (Qiu et al., 2007). These studies demonstrate the variable role of $\mathrm{CO}_{2}$ for this anthropophilic mosquito. This present study demonstrates the role of $\mathrm{CO}_{2}$ in guiding mosquitoes to the entrance of a trap, and shows that skin odours assist the mosquitoes to navigate the relatively narrow trap entrance. At close range to the trap, $\mathrm{CO}_{2}$ no longer affects the insects, as both turbulent and homogeneous $\mathrm{CO}_{2}$ plumes do not affect the trap catch when skin odour is present.

\section{Acknowledgements}

The authors thank Frans van Aggelen, André Gidding and Leo Koopman for having reared the numerous mosquitoes used in this study. Hans de Boer is acknowledged for computing a macro function to analyze carbon dioxide readings. 
然 


\section{Chapter 4}

\section{A 3D analysis of flight behaviour of Anopheles gambiae sensu stricto malaria mosquitoes in response to human odour and heat}

Jeroen Spitzen,

Cornelis W. Spoor,

Fabrizio Grieco,

Cajo ter Braak, Jacob Beeuwkes, Sjaak P. van Brugge, Sander Kranenbarg, Lucas P. J. J. Noldus, Johan L. van Leeuwen, Willem Takken 
Female mosquitoes use odour and heat as cues to navigate to a suitable landing site on their blood host. The way these cues affect flight behaviour and modulate anemotactic responses, however, is poorly understood. We studied in-flight behavioural responses of females of the nocturnal malaria mosquito Anopheles gambiae sensu stricto to human odour and heat. Flight-path characteristics in a wind tunnel (flow $20 \mathrm{~cm} / \mathrm{s}$ ) were quantified in three dimensions. With wind as the only stimulus (control), short and close to straight upwind flights were recorded. With heat alone, flights were similarly short and direct. The presence of human odour, in contrast, caused prolonged and highly convoluted flight patterns. The combination of odour + heat resulted in longer flights with more landings on the source than to either cue alone. Flight speed was greatest (mean groundspeed $27.2 \mathrm{~cm} / \mathrm{s}$ ) for odour + heat. Odour alone resulted in decreased flight speed when mosquitoes arrived within $30 \mathrm{~cm}$ of the source whereas mosquitoes exposed to odour + heat maintained a high flight speed while flying in the odour plume, until they arrived within $15 \mathrm{~cm}$ of the source. Human odour evoked an increase in crosswind flights with an additive effect of heat at close range $(<15 \mathrm{~cm})$ to the source. This was found for both horizontal and vertical flight components. However, mosquitoes nevertheless made upwind progress when flying in the odour + heat generated plume, suggesting that mosquitoes scan their environment intensively while they progress upwind towards their host. These observations may help to improve the efficacy of trapping systems for malaria mosquitoes by (1) optimizing the site of odour release relative to trap entry and (2) adding a heat source which enhances a landing response. 


\section{A 3D analysis of mosquito flight}

\section{Introduction}

Insect flight is mediated by a wide range of sensory cues (Cardé \& Bell, 1995; Cardé \& Willis, 2008). During upwind flight, these may, e.g., induce a visually-guided, odourmodulated anemotaxis, which aids the insect in reaching the source of a stimulus. For the nocturnal African malaria mosquito, Anopheles gambiae Giles sensu stricto (hereafter referred to as An. gambiae), chemical cues are thought to be most important, directing the insect upwind from a distance to a blood host (Gillies, 1980; Takken, 1991; Takken \& Knols, 1999). Chemical stimuli from the host may inform the mosquito not only about the location of the host, but also of its quality (Verhulst et al., 2011). Mosquitoes can be attracted by (host-) odours alone (Lacey \& Cardé, 2011; Qiu et al., 2011; Takken \& Knols, 1999), but surrounding visual and mechanical cues are important for determining flight direction in insects, even for nocturnal species under low light conditions (Gibson, 1995).

For many insects, flight is affected by pheromones, released from an approximate point source (David \& Birch, 1989; Mafra-Neto \& Cardé, 1994). The insect reaches the source by navigating upwind while making reiterative contact with packets of odour in a relatively narrow odour plume (Mafra-Neto \& Cardé, 1995a). The physical properties of the plume depend of course strongly on the wind conditions. Insects may also respond to blends of kairomones (which originate from one or more other species than that of the receiver). Examples are hematophagous mosquitoes, sandflies, biting midges and tsetse flies (Bhasin et al., 2001; Faiman et al., 2011; Gibson et al., 1991; Gibson \& Torr, 1999; Krcmar et al., 2005; Takken, 1991), and herbivorous insects such as common fruit flies, aphids and Colorado potato beetles, which navigate in a broad plume of odour-laden air, facilitating upwind orientation (Budick \& Dickinson, 2006; Visser \& Avé, 1978; Webster, 2012). Kairomones often originate from a wider source than pheromones, resulting in a much broader plume even close to the source. These differences may also affect fine-scale characteristics of the odorants in the plume, and affect the insect behavioural response.

Odour composition influences the efficacy of host-seeking behaviour during upwind odour-modulated flight in the mosquitoes Culex quinquefasciatus Say, Cx. tarsalis Coquillet and Aedes aegypti L. (Braks et al., 2005; Dekker et al., 2005). For example, odour composition affects the frequency and extent of crosswind flight behaviours in these species at certain distances from the source. For An. gambiae, however, it is not clear how olfactory cues affect flight characteristics and therefore hostfinding efficacy (Cardé \& Gibson, 2010; Cardé \& Willis, 2008). In the absence of olfactory cues, it was found that two Anopheles species tended to fly upwind in an approximately straight path (Takken et al., 1997). 


\section{Chapter 4}

The sensory cue heat elicits probing behaviour in Aedes mosquitoes, but its precise role in host-seeking remains unclear (Howlett, 1910). Heat also affects the mosquito at close proximity to the host (Laarman, 1958), but whether the addition of heat enhances host finding remains unresolved because its effect on mosquito behaviour has often been studied in combination with moisture and odour (Olanga et al., 2010). For example $C x$. quinquefasciatus flew less directly upwind when exposed to human odour plus heat compared to its flight pattern in clean air (Lacey \& Cardé, 2011). Radiated heat from a source can only be detected at close range (Healy et al., 2002; Peterson \& Brown, 1951), because the effect of the radiated heat on air temperature declines rapidly with distance. In contrast, odours can be detected from a much greater distance from the host (Cardé \& Gibson, 2010; Gillies \& Wilkes, 1970; Healy \& Copland, 2000). The combined effect of odour and heat on mosquito behaviour has rarely been studied. Details of in-flight characteristics when the insects are exposed to both stimuli simultaneously may elucidate behaviours that explain how both stimuli interact in guiding mosquitoes to their blood source.

Advances in automated tracking tools have made it possible to investigate the behaviour of nocturnal insects such as An. gambiae in great detail (Spitzen et al., 2008a). The aim of the current study was to elucidate characteristics of the hostseeking process of the malaria mosquito An. gambiae that can be exploited to develop species-specific trapping methods (Mukabana et al., 2012). Flight characteristics of mosquitoes exposed to host stimuli were studied by 3D tracking individual insects while they navigated through a plume of host-emitted cues in a wind tunnel under nocturnal conditions. We present a quantitative analysis of the flight response of $A n$. gambiae in the presence of a source of either human odour or heat, or a combination of the two stimuli. We expected that flight speed and angles of approach would vary with the distances from the source (either odour, heat or a combination) and the location of the insect with respect to the (approximate) extent of the odour plume. The detailed flight analysis provided insight into key characteristics that shape the behavioural response of a nocturnal mosquito to host-specific cues.

\section{Material and Methods}

Mosquitoes

The An. gambiae colony at Wageningen University, the Netherlands, originated from Suakoko, Liberia in 1987 and has been reared on human blood since 1988. Ethical approval for blood feeding was not requested as the authors did not consider this to be subject to the Dutch Act of Medical Research involving Human Subjects (WMO). Blood feeding was considered to cause a medium risk of allergic reaction and provision was in place that individuals were excluded if they reacted strongly 
to bites. In our anopheline mosquito cultures, no experimental infections took place and mosquitoes were free of any parasite. The colony was maintained at $27 \pm 1^{\circ} \mathrm{C}$, $70 \pm 5 \%$ R.H., and a light cycle of LD12:12h. Adults were fed with a $6 \%$ glucose solution ad libitum and offered a blood meal twice a week for 10 min. Females laid eggs on wet filter paper and these were transferred to water trays before hatching. Larvae were fed Tetramin $®$ (Tetrawerke, Melle, Germany) fish food daily.

Wind tunnel and air treatment system

All experiments were conducted in a wind tunnel $(160 \mathrm{~cm}$ long and $60 \times 60 \mathrm{~cm}$ across), supplied with air by a computer-controlled air treatment system (Figure 1 and Figure 2) at $27 \pm 1{ }^{\circ} \mathrm{C}, 70 \pm 3 \%$ relative humidity and a wind speed of $20.0 \pm$ $1.0 \mathrm{~cm} / \mathrm{s}$. A black epoxy metal mesh screen covered both ends of the wind tunnel. A glass funnel (diameter of straight tube, $4.3 \mathrm{~cm}$; diameter at open top, $10.5 \mathrm{~cm}$ ) was used to create an odour plume that was positioned in the centre of the screen at the upwind end of the tunnel. A cylindrical brass heating element was placed horizontally inside the funnel. Mosquitoes were released individually from a device placed in the centre of the downwind screen. For details see supporting information Text S1.

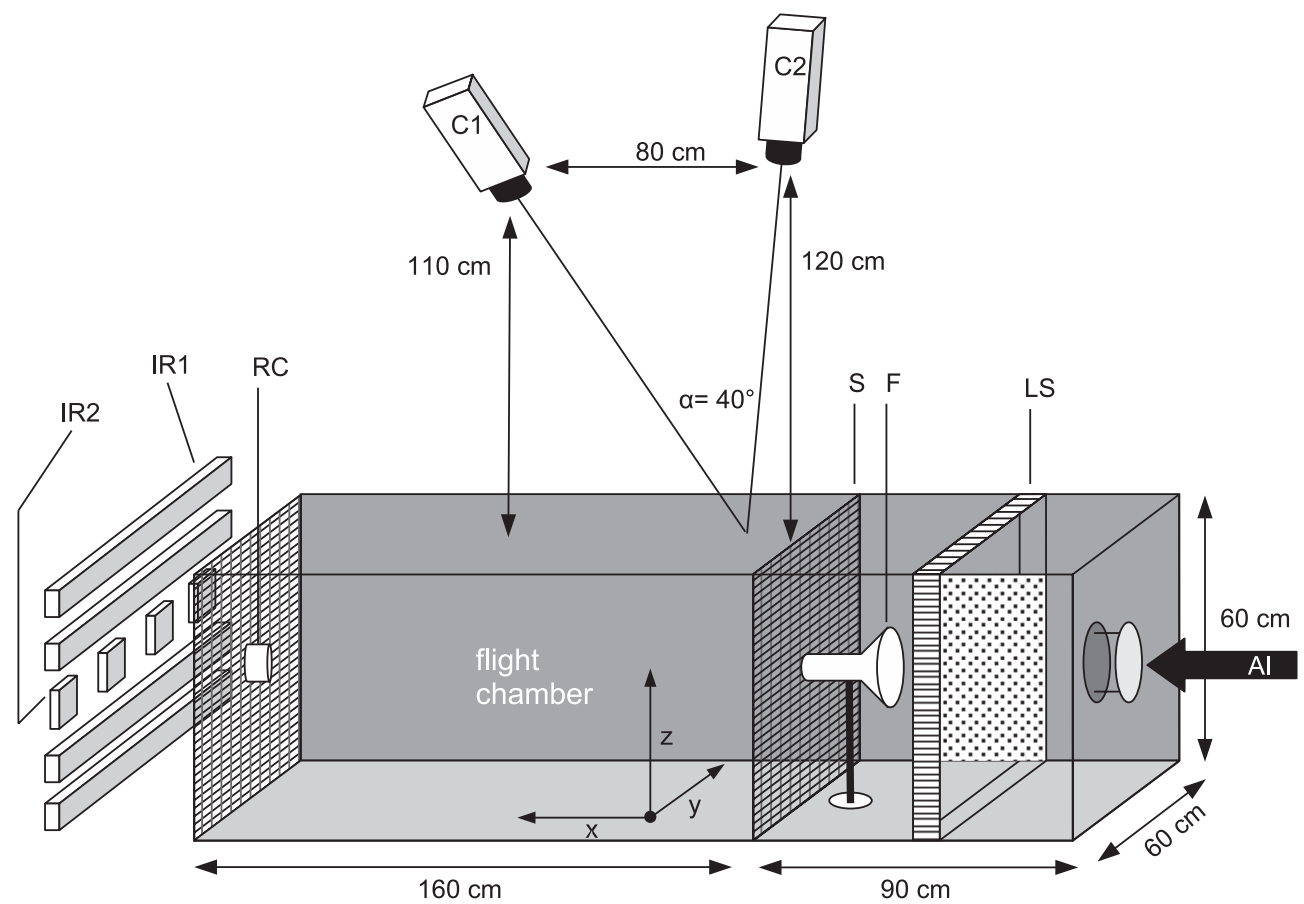

Figure 1. Schematic diagram of wind tunnel. Air inlet (AI), lamination screen (LS), glass funnel containing heat element $(F)$, mesh screen $(S)$, release cup $(R C)$, cameras $(C 1,2)$, IR lights type 1 (IR1), IR lights type 2 (IR2). The IR2 lights were operated by setting the accompanying adaptors at 9 Volts. 


\section{Chapter 4}

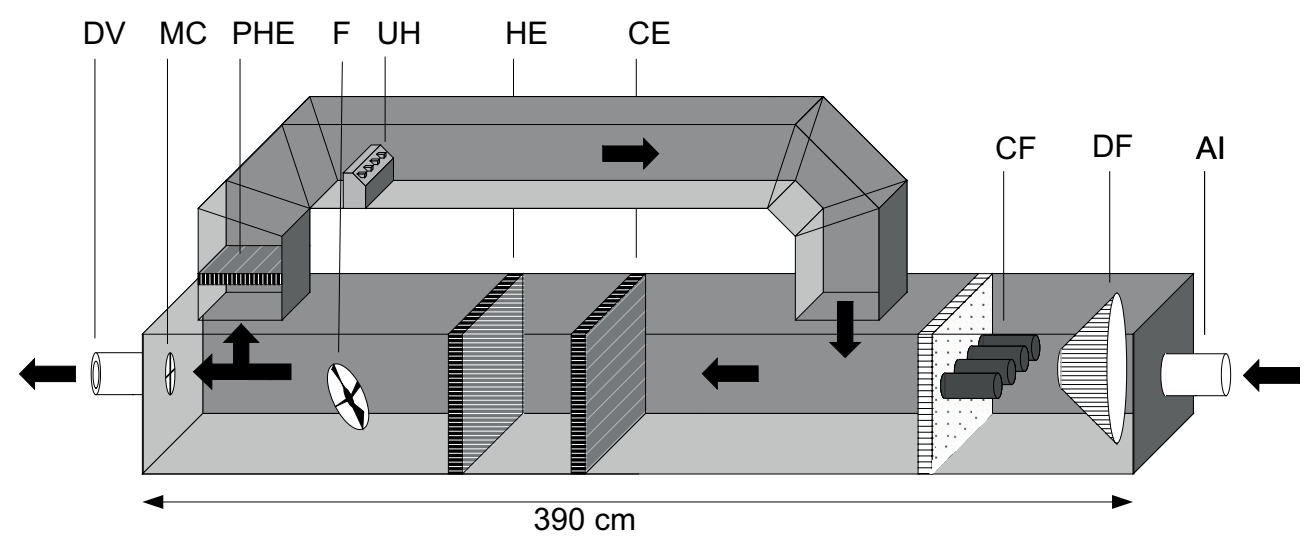

Figure 2. Schematic diagram of air treatment system. Air inlet (AI), fine dust filter (DF), charcoal filter cartridges (CF), cooling element (CE), heating element $(\mathrm{HE})$, ultrasonic humidifier $(\mathrm{UH})$, fan $(F)$, preheating element (PHE), measuring cross (MC), diaphragm valve (DV).

\section{D Flight recording}

Infrared (IR) light units were placed at the downwind end of the wind tunnel, facing upwind. The reflection of IR light from the mosquitoes' wings was filmed with two Cohu 4722-2000/0000 monochrome CCD video cameras (Cohu, San Diego, CA, USA) with Fuji non-tv f1.4 9 mm lenses, synchronized within $0.01 \mathrm{~s}$. MPEG-4 videos (PAL video: $704 \times 576$ pixels interlaced at 25 frames/s) were obtained for offline tracking. A software package, "Track3D" (Noldus Information Technology, Wageningen, The Netherlands) was developed as an add-on tool to EthoVision 3.1 to produce 3D track data for the position of a mosquito every $0.04 \mathrm{~s}$ from the $2 \mathrm{D}$ coordinates obtained from the two cameras. The air velocity in the wind tunnel was set at $20.0 \mathrm{~cm} / \mathrm{s}$ in the positive $x$ direction. The flight velocity was calculated by time differentiation of a spline function through a sequence of target positions (block), see supporting information Table S1. A flight angle of 0 degrees is defined as exact upwind flight. Speed is defined as the magnitude of the velocity vector in the $3 \mathrm{D}$ space and presented in a coordinate system that is fixed to the boundaries of the flight arena. Angular change is the change in the angle in space formed by successive data points. The mean angular velocity over the sample time is the angular change of the velocity vector times the sample frequency. We identified sequences of flight tracks that occurred a) within the defined space of the odour plume, 2) within a transition zone of $2.5 \mathrm{~cm}$ directly outside the plume, or 3 ) outside the transition zone (i.e., in notionally 'clean' air). The odour plume was approximated as a cone in space. Its dimensions were estimated by releasing smoke produced by a Safex® fog generator, F2010 plus (SafexChemie Gmbh, Schenefeld, Germany) using perfume-free fog fluid. The estimated 


\section{A 3D analysis of mosquito flight}

extent of the odour plume was defined by the apex, a point on the axis, and the cone angle of the funnel described above. Plume dimensions were estimated for treatments both with and without a heat source, while correcting for the effects of convection currents indicated by visualization experiments with smoke. The cone description is an approximation, as its boundaries are in fact variable. Details of computational methods used to produce 3D track data are provided in the supporting information, Text S2.

\section{Experimental procedure}

Individual 5-7 d-old female mosquitoes, which had not received a blood meal, were transferred to release containers $14-16 \mathrm{~h}$ before testing. A water-soaked cotton wick was placed in the container to prevent desiccation of the mosquito. Experiments were conducted during the last 4 hours of the scotophase. Mosquitoes that were not within the field of view of both cameras within 3 min after release were recorded as 'no response'. Recordings were stopped $10 \mathrm{~min}$. after the first entry of the mosquito within camera view or earlier if the mosquito landed on a wall or the upwind screen for $\geq 3$ s. Mosquito response was categorized as either 'no response', 'landing on upwind screen', landing at 'the source' or landing 'elsewhere' in the arena. A new mosquito was used for each bioassay. Surgical gloves were worn to avoid contamination of the experimental equipment.

\section{Stimuli}

Mosquito flights were recorded in the presence of combinations of four stimuli; 'No odour, no heat' was tested as the control, with a clean nylon sock placed in the glass funnel and the heat element switched off. The same set-up was used for the treatment 'heat', but with the heat element set at $34^{\circ} \mathrm{C}$ to mimic human skin temperature. Thirdly, 'odour' was tested by using a nylon sock (worn for $24 \mathrm{~h}$ by JS), containing foot odours (Njiru et al., 2006). The combination of odour and heat was designated 'odour + heat'. Female An. gambiae are attracted to human volatiles present on a nylon sock (Njiru et al., 2006; Smallegange et al., 2010a). To minimize variation in odour over days, the sock was re-used and stored in a freezer between testing days. Each day, treatments were changed after testing five mosquitoes. The testing order was randomized between and within days to rule out effects of testing sequence.

\section{Data analysis}

An area of $60 \times 60 \times 60 \mathrm{~cm}$ at the upwind end of the wind tunnel was in view of both cameras. Data points acquired $\leq 2 \mathrm{~cm}$ from the walls, including the upwind screen, were discarded for analysis because these boundaries may have affected 


\section{Chapter 4}

flight parameters. Track duration was defined as the total time a mosquito was within the field of view of both cameras and within the area of interest, thus excluding flight within $2 \mathrm{~cm}$ of boundaries. A Chi-square test was used to examine differences in response between treatments. A two-tailed t-test with unequal variance served to compare the proportion of time spent in different 'zones' of the flight arena: inside the plume, the transition zone or outside the plume for mosquitoes that landed in the proximity of the odour + heat source on the upwind screen $(5.0 \mathrm{~cm}$ diameter) with those landing elsewhere on the upwind screen.

Differences in flight parameters between treatments or between different 'sections' of $15 \mathrm{~cm}$ increments along the $x$ axis from the upwind screen within a treatment were tested with a GLM, Tukey $(\mathrm{T})$ or Games Howell test depending on the equality of variances (SPSS 19.0.1 for Windows). Non-normal data such as the proportion of time in a certain zone or angular velocity magnitude were tested with a Mann-Whitney $\mathrm{U}$ (two groups) and Kruskal-Wallis test (four groups). Mosquitoes that did not land on the upwind screen were excluded from the analysis that tested differences between the sections. Differences were analysed only for flights containing a minimum of 20 recorded positions of the mosquito in the two image planes. This was reduced to six positions for the analysis between the $15 \mathrm{~cm}$ sections, where fast (up to $75 \mathrm{~cm} / \mathrm{s}$ straight upwind) flying mosquitoes would otherwise be excluded from the results. An independent sample t-test was used to test for differences between flight speeds in- and outside the plume. The square root of this variable was taken to normalize the data.

Comparisons between flying in- and outside the plume (with exclusion of the transition zone) were only done for the treatment odour + heat, as for the other treatments there were insufficient data for within-plume flights. The change in flight speed was analysed in more detail with a paired sample t-test by comparing the average flight speed of 10 frames before entering the transition zone around the plume with the following 10 frames after the transition zone. The distance to the upwind screen when entering a plume was compared with the distance at exiting (and vice versa) and tested for significant differences using a Wilcoxon test.

Distribution of flight directions was presented in rose diagrams and analysed for the $x y$ and $x z$ plane over $3^{\circ}$ bins and tested for significant differences between treatments using GLMs. Rose diagrams were computed by using the weighted mean absolute cosine and absolute sine across bins, with the sum of velocities of each mosquito in each bin as weights. Parallel to the $x y$ plane this absolute sine measures the frequency of crosswind flight and the absolute cosine the frequency 


\section{A 3D analysis of mosquito flight}

of up/down wind flight. The ratio of absolute sine and cosine (the absolute tangent) expresses the degree of crosswind manoeuvers. A high absolute tangent indicates strong crosswind movement and a low one dominant up/down- wind fly movement. The tangent was analysed in three ways. As the experiment had a $2 \times 2$ factorial design of factor "odour" and factor "heat", both with two levels (no, yes), the first analysis was a GLM of an ANOVA type and examined the main effect of odour (averaging over heat levels) and of heat (averaging over odour levels) and the interaction between odour and heat which measures whether the odour effect depends on the heat level. As the interaction was non-significant, the combined effect of heat and odour was the sum of the main effect of odour and that of heat. Secondly, paired t-tests were performed to compare the absolute tangent of flight paths in plume vs. outside plume for A) all mosquitoes exposed to odour + heat and B) the mosquitoes that landed on the odour + heat source. Third, a linear mixed model analysis was performed to study whether the mosquitoes that landed in the proximity of the source showed a different flight path than mosquitoes that did not. This was done irrespective of the mosquito being in or outside the plume, while correcting for a presumed effect of being in or outside plume.

\section{Results}

Flight tracks were reconstructed into $3 \mathrm{D}$ images as shown in Figure 3. As a considerable proportion of mosquitoes exhibited displacements both in $x y$ and $x z$ planes, a 3D analysis was required to estimate behavioural parameters with sufficient accuracy. The trajectories showed that without a host cue, many mosquitoes flew upwind, along the $x$ axis, with relatively little deviation in the $y$ - and $z$ axis, followed by landing on the upwind screen (Figure $3 A-C$ ). With heat alone, flights were similarly short and direct (Figure 3D-F). The presence of human odour, by contrast, caused longer and highly convoluted flight patterns (Figure 3G-L).

The reconstructed plume was cone shaped with an estimated cone angle of $20^{\circ}$. With heat $\left(34^{\circ} \mathrm{C}\right)$, the angle of the plume relative to the floor was estimated to be $13^{\circ}$. To account for variations in plume eddies, a transition zone of $2.5 \mathrm{~cm}$ from plume to outside plume was defined and data points of mosquitoes flying in this zone were excluded from the analysis concerning 'in plume' or 'outside plume' behaviour. Temperature readings showed that the heat element heat affected air temperature up to $20 \mathrm{~cm}$ downwind from the source (Figure S1).

Of the 201 mosquitoes tested, 156 (78\%) responded by flying upwind and 145 of the tracks were recorded and used for analyses. An upwind flight response varied from $65 \%$ to $85 \%$ (Figure 4 ) and was not significantly different between the treatments 


\section{Chapter 4}
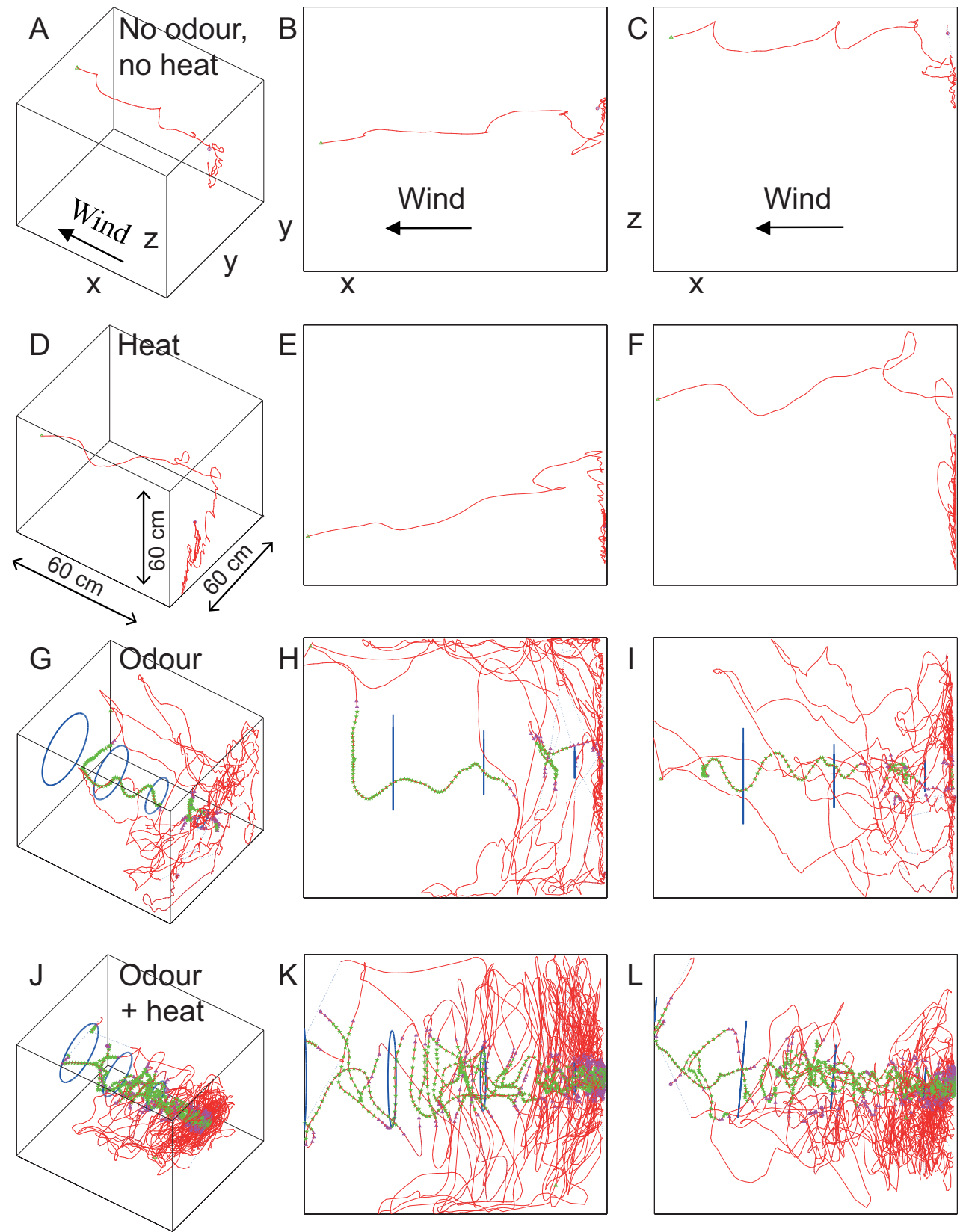

Figure 3. Examples of flight tracks of Anopheles gambiae s.s. for each treatment viewed from different angles. Each treatment represents a single female. A - C clean acclimatized air only (control, $9 \mathrm{~s}), \mathrm{D}-\mathrm{F}$ heat $(21 \mathrm{~s}), \mathrm{G}-\mathrm{I}$ human odour (112 s) and J - L human odour + heat (231 s). Red dots indicate samples outside the cone and transition zone, magenta triangles are used for samples within the transition zone and green stars indicate that the insect is tracked within the defined odour plume. Mosquitoes that landed near the centre of the upwind screen, within a circle with a diameter of $5 \mathrm{~cm}$, were recorded as landing on the source. 


\section{A 3D analysis of mosquito flight}

$\left(X^{2}, P=0.076\right)$. Human odour alone caused the lowest flight response (65\%) within 3 min., and up to $67 \%$ of all responding mosquitoes landed on the upwind screen. Odour + heat elicited landing within $5 \mathrm{~cm}$ of the centre of the source for $46.5 \%$ of the responding mosquitoes, which is more than the other treatments, in which $0-7 \%$ of the mosquitoes landed at the source $\left(X^{2}, P<0.05\right.$, Figure 4). The percentage of landings on the odour + heat source was similar during the first five (41\%) and the last five testing days (48\%), indicating that the storage and re-use of the worn sock did not affect responses over time $\left(X^{2}, P=0.458\right)$. Mosquitoes exposed to odour + heat that landed on the source $(n=20)$ spent significantly more time flying than mosquitoes that landed elsewhere on the upwind screen $(n=99 ; 99 \pm 23 \mathrm{~s}$ vs. $17 \pm$ $3.2 \mathrm{~s}$, Mann Whitney $U$ test, $P<0.001$ ).

Flight tracks were longer if mosquitoes were exposed to human odour + heat compared to heat alone or the control (Table 1, GLM, $P<0.05$ ). The percentage of time spent inside the plume was greater for mosquitoes that landed on the odour + heat source than those exposed to the same stimuli, but landing elsewhere on the upwind screen (Table 2, two-tailed $t$-test, $P<0.01$ ).

\section{Flight direction}

Vertical and horizontal crosswind flights were common for all treatments when mosquitoes flew close to the upwind screen. At distances $>15 \mathrm{~cm}$ from the upwind screen, linear up- and downwind movements dominated for all treatments other than treatment odour + heat (Figure S2 and Figure S3). For the latter, crosswind flights

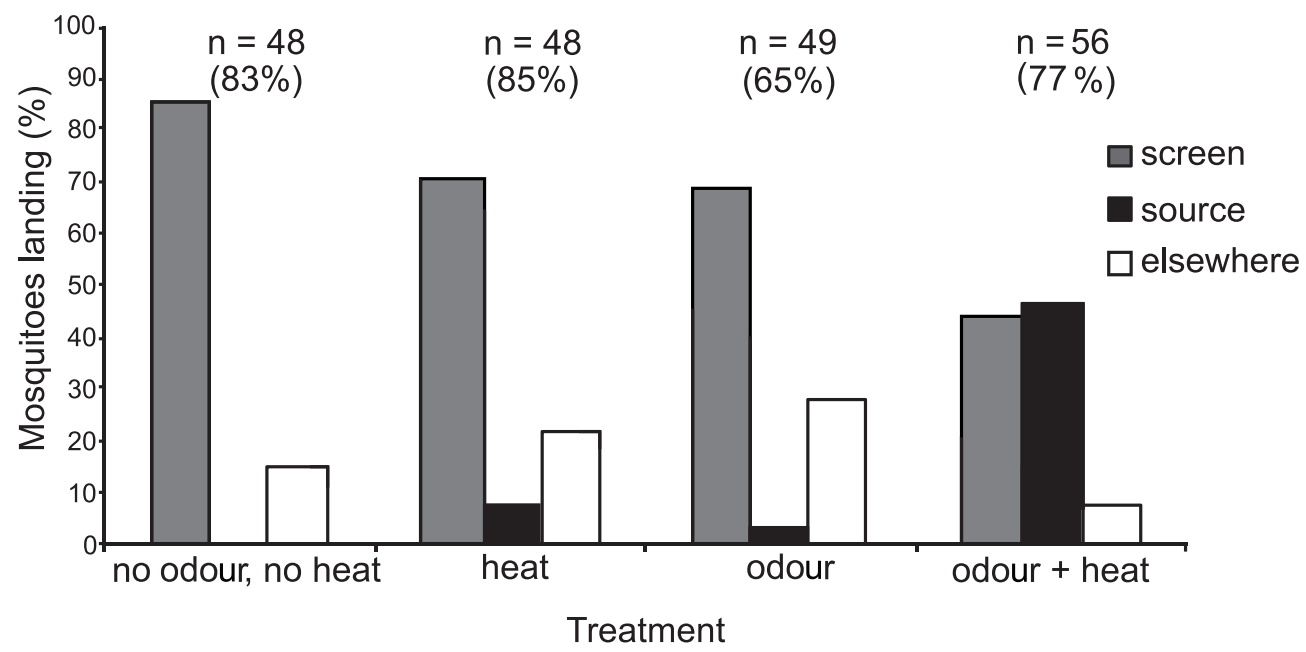

Figure 4. Mosquito responses with four different treatments. The percentage of responding mosquitoes landing on the upwind screen, source, or elsewhere in the arena per treatment. $n=$ Number of mosquitoes tested. Percentage $(\%)=$ percentage of mosquitoes leaving the release site within 3 min. 


\section{Chapter 4}

dominated, especially $15-30 \mathrm{~cm}$ from the upwind screen, and further away from the source, these mosquitoes more often expressed downwind-directed flights than those exposed to one of the other three treatments. Human odour contributed most to the observed crosswind movements (Table S2) and this was especially apparent for horizontal $(x y)$ movements and significant $(P<0.001)$ for all sections. Close to the source $(<15 \mathrm{~cm})$, the change in crosswind behaviour was also influenced by heat. For vertical $(x z)$ flights, this change was, within each '15 cm' section, significantly affected by odour but not by heat. There was no interaction between the treatments odour and heat $(P>0.05)$. Because both cues caused a positive effect on the absolute tangent, odour + heat showed, of all treatments, the largest increase in crosswind manoeuvers compared to the control. This is summarized in Figure 5 where the mean ( \pm s.e.m.) tangent is presented per section for the horizontal and vertical plane.

The mean magnitude of angular velocity for upwind flight was not different between any of the treatments for upwind flight (GLM, $P=0.057$, Table 1) and for downwind flight (GLM, $P=0.480)$. When all treatments are pooled, mosquitoes flew downwind with a greater mean magnitude of angular velocity compared to upwind flights (653.4 $\pm 19.7(n=94)$ vs. $524.8 \pm 14.9(n=135)$ deg/s, respectively; Mann-Whitney U test, $P<0.001)$. Mosquitoes had the highest mean angular velocity magnitude when at < $15 \mathrm{~cm}$ from the upwind screen. (Kruskal-Wallis, $P<0.001$ ).

During upwind flight, the exposure to odour + heat led to more crosswind movements outside than inside the plume (Table $3 \mathrm{~A}$ ). This was most apparent at $<30 \mathrm{~cm}$ from the screen but not significantly different between the $15 \mathrm{~cm}$ sections. The same trend was visible for mosquitoes that landed on the source (Table 3B). Mosquitoes landing on the odour + heat source expressed overall more crosswind behaviour than those landing elsewhere on the screen irrespective of flying in or out the plume (Table $3 \mathrm{C}$ ).

Table 1. Mean ( \pm s.e.m.) track duration, mean up- and downwind flight velocity components and magnitude of angular velocity of mosquitoes that flew upwind in the defined arena exposed to four different treatments.

\begin{tabular}{lllllllll}
\hline \multirow{2}{*}{ Treatment } & \multicolumn{2}{l}{ track duration } & \multicolumn{2}{l}{ speed upwind } & \multicolumn{2}{l}{ speed downwind } & \multicolumn{2}{l}{ ang. velocity for upw. flight } \\
\cline { 2 - 10 } & $\mathrm{n}$ & $(\mathrm{s})$ & $\mathrm{n}$ & $(\mathrm{cm} / \mathrm{s})$ & $\mathrm{n}$ & $(\mathrm{cm} / \mathrm{s})$ & $\mathrm{n}$ & $(\mathrm{deg} r e e s / \mathrm{s})$ \\
\hline no odour, no heat & 33 & $15.2 \pm 9.8 \mathrm{a}^{2}$ & 30 & $22.7 \pm 1.2 \mathrm{a}$ & 18 & $20.4 \pm 1.7 \mathrm{a}^{1}$ & 30 & $465.9 \pm 31.3 \mathrm{a}^{2,3}$ \\
heat & 41 & $18.8 \pm 8.8 \mathrm{a}^{2}$ & 39 & $24.7 \pm 1.0 \mathrm{a}$ & 25 & $25.4 \pm 1.5 \mathrm{ab}^{1}$ & 39 & $556.9 \pm 27.4 \mathrm{a}$ \\
odour & 32 & $32.7 \pm 9.9 \mathrm{ab}^{2}$ & 30 & $24.3 \pm 1.2 \mathrm{a}$ & 24 & $25.9 \pm 1.5 \mathrm{~b}^{1}$ & 30 & $508.1 \pm 18.5 \mathrm{a}$ \\
odour + heat & 39 & $67.3 \pm 8.9 \mathrm{~b}^{2}$ & 36 & $25.9 \pm 1.1 \mathrm{a}$ & 27 & $27.2 \pm 1.4 \mathrm{~b}^{1}$ & 36 & $553.0 \pm 27.8 \mathrm{a}$
\end{tabular}

Flight speed is defined as the mean speed relative to the boundaries of the wind tunnel overall time intervals with an upwind and downwind velocity component. Wind speed was $20 \mathrm{~cm} / \mathrm{s}$. Different letters within a column indicate significant differences between treatments $(\mathrm{GLM}, \mathrm{P}<0.05) . \mathrm{n}=$ Number of included flights.

${ }^{1}$ Equal variances Tukey $(\mathrm{T})$

'Unequal variances Games Howell

${ }^{3} \mathrm{P}=0.057$ 


\section{A 3D analysis of mosquito flight}

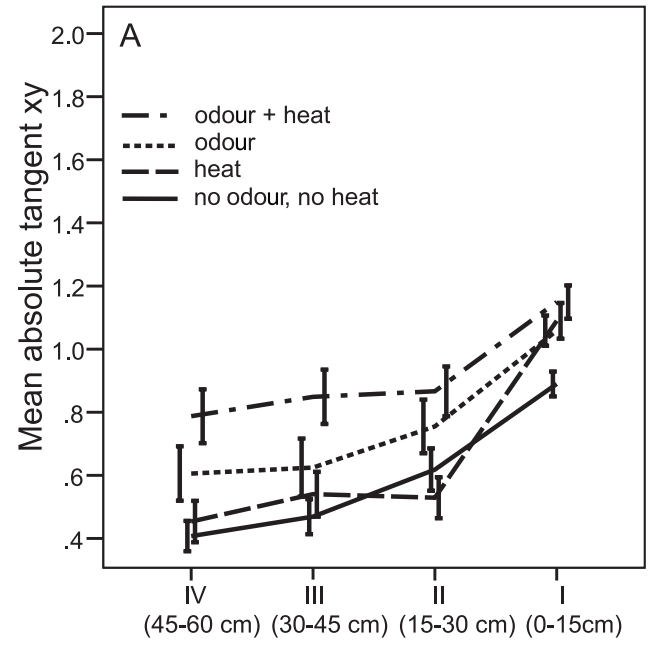

Section

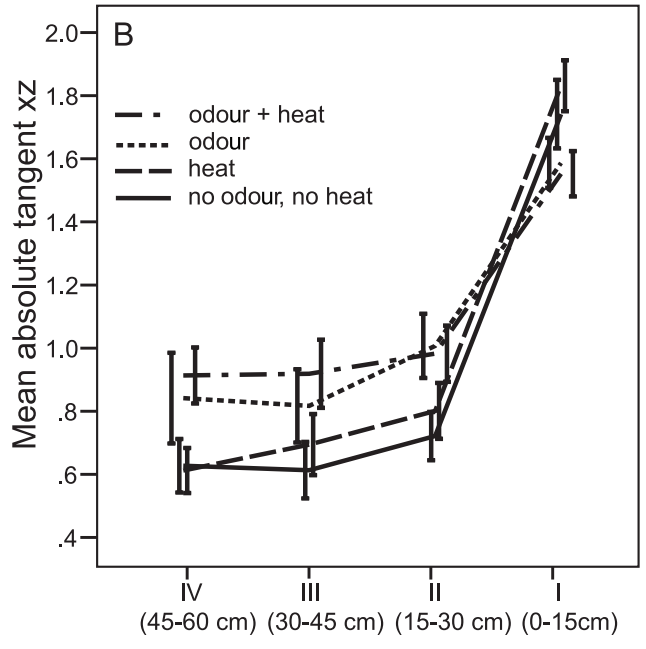

Section

Figure 5. The degree of crosswind flight plotted for the horizontal (xy) and the vertical (xz) plane. Mean ( \pm s.e.m.) absolute tangent of mosquito flight paths at four different sections from the upwind screen for each treatment including the control (no odour, no heat).

This was observed for all spatial sections and for both horizontal and vertical flight components except for the $x z$ plane in section I $(<15 \mathrm{~cm})$.

By comparing their $x$-positions from the moment mosquitoes entered until they exited the plume it was found that the insects made upwind progress when flying in the plume (Figure $6 \mathrm{~A}$, Wilcoxon, $\mathrm{Z}_{-6.921}, \mathrm{P}<0.001$ ). This was observed for mosquitoes that reached the source and also for those that landed elsewhere on the upwind screen (data not shown). No directional bias was observed after comparing the $x$-position when outside the plume until (re-)contacting the plume (Figure 6B, Wilcoxon, $\mathrm{Z}_{-0.669}$, $P=0.504$ ), which confirms our data of increased crosswind behaviour for flights outside the plume (e.g. Table 3). The mosquitoes still flew forward with respect to the air when outside the plume because we have considered ground speeds. We refer to the supporting information, Table S3, for the mean upwind progress per mosquito.

Table 2. Mean ( \pm s.e.m.) percentage of time mosquitoes spent inside the plume, in the transition zone or outside the plume boundaries as defined for the treatment odour + heat.

\begin{tabular}{|c|c|c|c|c|}
\hline \multirow[t]{2}{*}{ mosquitoes landing: } & \multirow[t]{2}{*}{$\mathrm{n}$} & \multicolumn{3}{|c|}{ mean $(\%)$ of time spent flying in a zone of the odour plume } \\
\hline & & inside plume & transition zone & outside plume \\
\hline on the source & 16 & $30.5( \pm 3.5)$ a & $8.1( \pm 0.7) \mathrm{a}$ & $61.4( \pm 3.9)$ a \\
\hline on upwind screen, away from the source & 19 & $7.3( \pm 2.9) \quad b$ & $3.8( \pm 1.0) b$ & $88.9( \pm 3.8) b$ \\
\hline
\end{tabular}

$\mathrm{n}=$ Number of included flights. Different letters in the same column express significant differences in time spent in the corresponding section for mosquitoes that landed eventually on the source compared to mosquitoes that landed elsewhere on the upwind screen (two tailed t-test with unequal variance $(P<0.01)$ ). 


\section{Chapter 4}

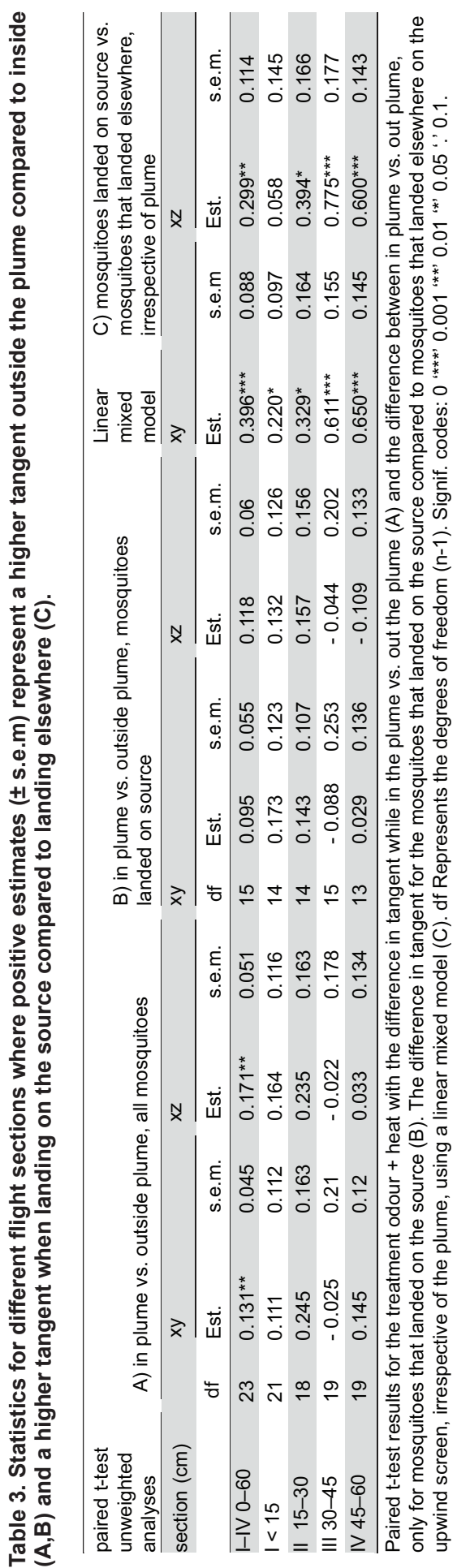




\section{A 3D analysis of mosquito flight}
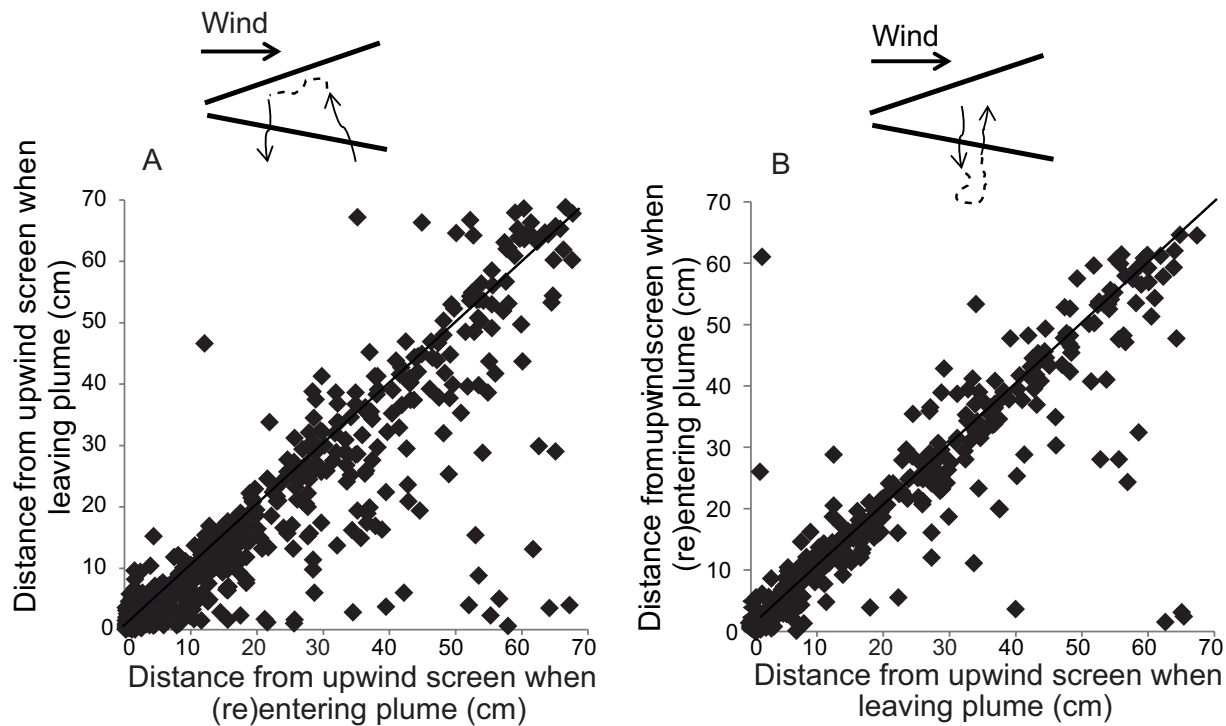

Figure 6. Displacement of 'odour + heat' treatment mosquitoes along the $x$ axis of the wind tunnel in relation to entering and leaving the plume. A - Distance from the upwind screen at the moment of entering the plume ( $x$ axis) and the moment of leaving ( $y$ axis). B - Distance from the upwind screen at the moment of flying outside the plume and (re-) contacting the plume. The figure represents all individuals $(n=16)$ that landed near the odour + heat source. The absolute number of occurrences per mosquito is given in table S3. The solid line represents a theoretical situation where net up- or downwind displacement between the moments of entering and leaving, or vice versa is equal.

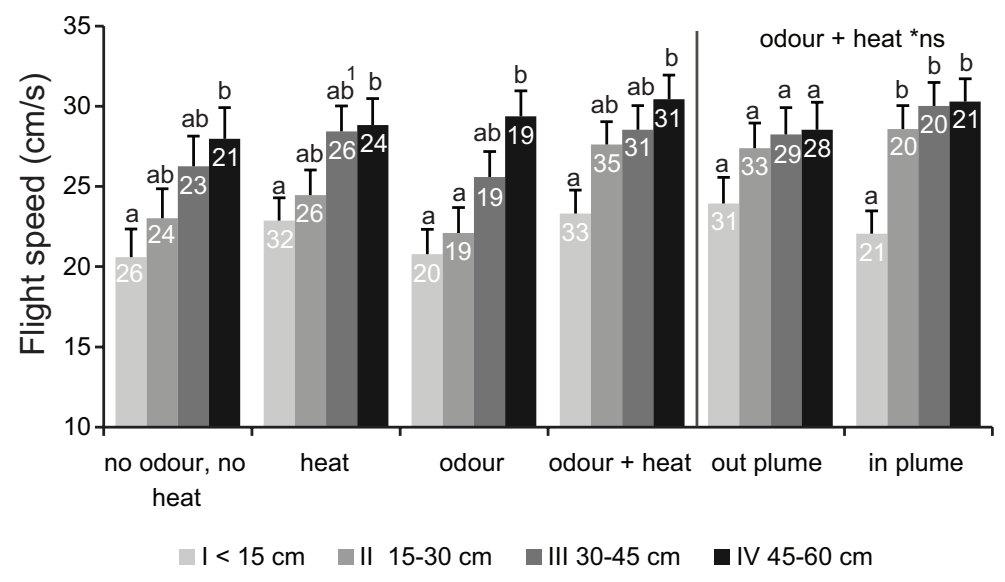

Figure 7. Mean ( \pm s.e.m.) flight speed $(\mathrm{cm} / \mathrm{s})$ over all time intervals with an upwind velocity component in different sections from the upwind screen.

Flight speed is relative to the boundaries of the wind tunnel. Wind speed was $20 \mathrm{~cm} / \mathrm{s}$. Different letters indicate significant differences between sections (GLM, Equal variances $\rightarrow$ Tukey (T), $P<0.05,{ }^{1} P=$ $0.051)$. Numbers inside bars represent the number of included flights. The flight speed for the treatment odour + heat is also presented while flying outside the plume and inside the plume, respectively. * Differences between out plume and in plume speed, within a distance section, were not significant (ns, independent sample t-tests, $P>0.05$ ). 


\section{Chapter 4}

\section{Flight speed}

Mosquitoes, pooled over all treatments, flew upwind with a mean speed relative to the ground of $24.5 \pm 0.6 \mathrm{~cm} / \mathrm{s}(\mathrm{n}=135)$, and downwind at $25.1 \pm 0.8 \mathrm{~cm} / \mathrm{s}(\mathrm{n}=$ 94). Flights of mosquitoes exposed to odour + heat $(27.2 \pm 1.2 \mathrm{~cm} / \mathrm{s}, \mathrm{n}=38)$ were significantly faster than those of control mosquitoes $(22.5 \pm 1.29 \mathrm{~cm} / \mathrm{s}, \mathrm{n}=30$, GLM, $P<0.05)$. The difference in flight speed between the treatments is mainly caused by downwind flights, with a lower flight speed of control mosquitoes than those exposed to odour + heat (Table 1, $P<0.05$ ). Within the different treatments, upwind flight speed was reduced when reaching the upwind screen (Figure 7). This was significant between the sections $0-15 \mathrm{~cm}$ and $45-60 \mathrm{~cm}$. Odour alone, however, also caused reduced flight speeds between the section $15-30 \mathrm{~cm}$ and $45-60 \mathrm{~cm}$. At $15-30 \mathrm{~cm}$, flight speed was significantly higher for odour + heat $(27.6 \pm 14 \mathrm{~cm} / \mathrm{s})$ than for the odour treatment $(22.1 \pm 1.6 \mathrm{~cm} / \mathrm{s}$, GLM, $P<0.05)$. For odour + heat, which elicited $46.5 \%$ of the mosquitoes to land near the source, mosquitoes maintained their relatively high flight speeds up to $15 \mathrm{~cm}$ from the upwind screen. A strong decrease in flight speed at $<15 \mathrm{~cm}$ was observed only for in-plume mosquitoes $(P<0.01$, Figure 7 ). At this distance the mean upwind flight speed was $23 \%$ lower than at 15 $30 \mathrm{~cm}$ from the screen and lower in the plume than outside it. For the three sections further downwind, the differences in flight speed were not significant (independent sample t-tests, $P>0.05)$. Insufficient replicates were available to examine flight speed per section within the plume for the other treatments. A considerable change in flight speed (either negative or positive) occurred upon entering or leaving the plume (paired sample t-test, $\mathrm{t}(141)=0.41, P=0.67$; $\mathrm{t}(169)=-0.53, P=0.60$, respectively). Mean flight speeds upon entering were overall $4.5 \mathrm{~cm} / \mathrm{s}$ higher than those at the moment of leaving the plume (GLM, $P<0.001$, visualized in Figure S4).

\section{Discussion}

The analysis of upwind odour-modulated anemotaxis of the nocturnal mosquito An. gambiae revealed significant differences in flight behaviour between the treatments odour, heat and odour + heat, especially at $<30 \mathrm{~cm}$ from the source. Without additional cues, mosquitoes took off for upwind flight as described by Cardé and Gibson and references therein (Cardé \& Gibson, 2010). Odour + heat caused a high landing response on the source. This was preceded by a longer, faster and more tortuous flight than with odour or heat treatments as single cues, suggesting that with the combination a more effective behaviour is induced, enabling the insect to locate the host more precisely. Human odour contributed most to the observed crosswind behaviour. Close to the source, heat also induced an increase in crosswind movements and flight speed was reduced. 


\section{A 3D analysis of mosquito flight}

\section{Flight direction}

Without odour, many insects fly crosswind or downwind (David, 1986). When odour is lost during upwind flight, insects generally exhibit casting behaviour, which is described as back and forth movements across the wind without making much progress upwind (Cardé \& Gibson, 2010; Cardé \& Willis, 2008). Upon restoration of contact with odour, they continue flying upwind (Mafra-Neto \& Cardé, 1995b). Tsetse flies, however, respond to odour plumes by turning sharply upwind when flying into a plume (Faiman et al., 2011; Gibson \& Brady, 1988). The mosquitoes Cx. tarsalis and $C x$. quinquefasciatus, when approaching an odour-baited trap, flew straight upwind until they overshot the odour source, at which point they engaged in more tortuous flight (Cooperband \& Cardé, 2006). When these mosquitoes approached a source of $\mathrm{CO}_{2}$, heat and moisture, the tortuosity of the flight path increased and speed reduced within $30 \mathrm{~cm}$ of the source. Increased tortuous flights in response to host stimuli are also described for Ae. aegypti (Dekker \& Carde, 2011) and Cx. quinquefasciatus (Lacey \& Cardé, 2011). This is similar to our observations with An. gambiae approaching odour + heat and may be a general behavioural pattern when mosquitoes arrive in proximity of a host. The increased crosswind behaviour suggests that upwind navigating host-seeking mosquitoes scan the arena before landing on the host. Mosquitoes that did not land on the source spent more time outside the plume (Table 2). Some of them spent little to no time in the plume and continued flying upwind, which may explain the results shown in Table 3C. This also suggests that the observed increase in crosswind manoeuvers while outside the plume (Table $3 A B$ ) was triggered by initial encounters with the plume. These findings validate a recent simulation study of mosquito flight behaviour in response to odour cues (Cummins et al., 2012). The authors suggest that the crosswind strategy led to the largest percentage of host finding, but that such flights took substantially longer. Our results are in agreement with these conclusions, as crosswind flights increased significantly in odour and led to longer flight trajectories.

\section{Flight speed}

Dekker et al. (2005) reported higher velocities for Ae. aegypti when exposed to skin odour or a human hand than to clean air. They mention that flight speed remained close to constant, irrespective of flying in or outside the plume. We observed a higher mean flight speed for the odour + heat treatment than for clean air. Flight speed decreased significantly in the vicinity of the odour + heat source (Figure 7). Lacey and Cardé (2011) found that flight speed of $C x$. quinquefasciatus was lower when exposed to heated foot odour compared to heat alone, but here flight speed varied within all treatments at different distances from the source. Our observed difference in average flight speed just after entering a plume compared to after leaving it suggests 


\section{Chapter 4}

that $A n$. gambiae mosquitoes reduce ground speed after entering the odour plume and fly more upwind. Flying more upwind reduces ground speed even if the mosquito maintains the same flight speed with respect to the air. With the current approach it takes more than 0.9 seconds before we can measure this change in flight speed (20 frames $=0.8 \mathrm{~s}+$ ca. $0.1 \mathrm{~s}$ in the transition zone). The proportion of time spent in the plume was significantly higher for mosquitoes that landed on the odour + heat source than for those that landed elsewhere on the upwind screen. Moreover, when the odour + heat source was approached, flight speed decreased and the angular velocity increased, suggesting that the insect made many turns per second to assess the exact location or quality of the odour plume, which is considerably narrower at this point than further downwind (Figure 3). Both behavioural changes are most likely an adaptation to prepare for a landing on or near the host (Healy et al., 2002). These results demonstrate that contact with odour causes a significant shift in behaviour, allowing the insect to more accurately assess the source of the host stimuli.

The behaviour of mosquitoes close to the upwind screen suggests that the screen acts as a physical barrier, which is sensed from a (short) distance without the need for making contact with it. We assume that this may be caused by a change in wind structure due to increased air turbulence around the mesh screen or visual cues from this screen.

The response to heat, either alone or combined with odours, has previously been studied with Aedes mosquitoes (Peterson \& Brown, 1951) and with An. gambiae (Healy et al., 2002; Olanga et al., 2010). In most studies, enhanced attractiveness was observed. However, the observations did not reveal whether heat activates odour components and therefore indirectly attracts more mosquitoes or that heat itself elicits a landing or trap entry response at close range. This last hypothesis is supported by Healy and Copland (Healy \& Copland, 2000), who reported increased landing responses when combining heat + volatizing acids such as 2-oxopentanoic acid. Heat is likely to affect the ratio in which odorants are released from the substrate, and hence their concentration in a plume depending on molecular characteristics and this may (partly) explain our observed differences between treatments odour and odour + heat.

In An. gambiae, odour alone caused longer flights and increased crosswind movements compared to the control, suggesting host-seeking behaviour. This effect was not observed for heat alone. This raises the question whether mosquitoes need to be in contact with host odour in order to respond to heat. An. gambiae have 


\section{A 3D analysis of mosquito flight}

heat-sensitive molecular receptors on the antennae (Wang et al., 2009), suggesting that mosquitoes use heat sensing for host-seeking and/or recognition in addition to odour and vision. With a wind speed of $20 \mathrm{~cm} / \mathrm{s}$, the effect of body heat faded to the background over a distance of $20 \mathrm{~cm}$, indicating that at greater distance from the host the principal cues for host orientation are odour and wind direction. This short range was also the distance over which Ae. aegypti was attracted to heat and moisture (Kellogg \& Wright, 1962). The higher flight speed observed for the treatment odour + heat at $15-30 \mathrm{~cm}$, compared to mosquitoes exposed to odour alone, may be explained by an increase of odour contact due to the effects of heat on breaking up the odour filaments.

The host-seeking strategy of An. gambiae may be explained as consisting of highly tortuous flights, initially evoked by odour, enhancing the probability of reaching the source further upwind, whereas heat causes a significant reduction in flight speed, allowing the insect to accurately locate the source of the stimuli by a series of convoluted flights, before landing. Similar to moths, which exhibit casting behaviour when losing an odour plume (Mafra-Neto \& Cardé, 1994; Vickers \& Baker, 1994), An. gambiae also expresses such behaviour when losing odour. Casting behaviour by mosquitoes, as demonstrated to occur in both $x y$ and $x z$ planes, is directed to lead the insects back into the plume. The dominant flight direction of mosquitoes upon plume entry is upwind (Figure 6A).

Anopheles gambiae females naturally orient themselves under nocturnal conditions while seeking for hosts indoors. The crosswind flights $15-30 \mathrm{~cm}$ from the source, by mosquitoes exposed to odour + heat (Figure S2 and Figure S3), suggest that the insects scan their environment intensively before they proceed with a landing response. This is a clear difference with diurnal mosquitoes, such as Ae. aegypti, which fly with less convoluted paths towards the host (Dekker \& Cardé, 2011). For other insects such as Drosophila melanogaster Meigen and Manduca sexta (Linnaeus) olfactory and visual responses are used interactively just before landing (Balkenius \& Dacke, 2010; Stewart et al., 2010; Vickers \& Baker, 1994). The lack of sufficient visual feedback in nocturnal endophilic insects such as An. gambiae may be responsible for a shift in cues which, at close range, guide them to their hosts. Lacey and Carde (2012) mention that prominent optomotor cues may indeed not affect flight orientation of the crepuscular mosquito $C x$. quinquefasciatus while moving upwind. It remains ambiguous whether it is ortho-kinesis to heat or a different set of semiochemicals that triggers An. gambiae to evoke a landing response. Most likely, it is a combination of the two factors (Cardé \& Gibson, 2010; Healy et al., 2002). 


\section{Chapter 4}

The development of synthetic odour blends for mosquitoes (Mukabana et al., 2012; Njiru et al., 2006; Smallegange et al., 2010a; Williams et al., 2006) in combination with 3D analysis of flight allows for detailed examination of the effect of individual olfactory components on behaviour and assessment of the contribution of each component at various distances from the source. Heat strongly interacts with olfactory cues, and therefore should be considered as a potential stimulating cue when developing mosquito trapping devices. The position of the odour release point relative to the suction fan should be investigated, in order to allow for optimal dispersal of the odorants. The addition of a heat source to an odour-baited trap is likely to enhance the landing and/or trap-entry response, rendering such traps more effective. We conclude that odour and heat both affect the upwind anemotaxis of host-seeking mosquitoes. Furthermore, the adaptive flight strategy, expressed by highly convoluted flight in combination with strongly reduced flight speed in the vicinity of the source, contributes to a successful completion of this foraging process.

\section{Acknowledgements}

We thank the following people for their contributions. Frans van Aggelen, André Gidding and Leo Koopman supplied the mosquitoes. The wind tunnel was constructed by Gradus Leenders, Evert Janssen, Eltje Groendijk and Ton van der Zalm. Andries Hofman and Valentina lo Verde created a macro function to analyse large data files. Jeremy Harbinson provided the thermocouple set-up. Piet Huisman laid the basis for the 3D tracking system. Ring Cardé contributed to valuable discussions. 


\section{A 3D analysis of mosquito flight}

\section{Supporting Information}

\section{S1: Material and Methods}

Air treatment system

An air treatment system and wind tunnel was developed and installed by Facility Services Tupola, Plant Sciences Group, Wageningen University and Research Centre (WUR), The Netherlands. The system ensured a constant flow of purified warm and humid air into the wind tunnel (Figure 1, main document). Air collected outdoors was led through a series of filters driven by a fan based on ECM technology (P. Lemmens Company N.V., Gembloux, Belgium). After a primary dust filter, the air passed an Opakfil Green® fine dust filter (Camfil Farr, La Garenne-Colombes, France). Four activated charcoal filter cartridges were used for further purification of the air. Pressure differences over filters were measured to monitor the filter conditions. Purified air was pre-heated by a heating element (Bo-air, Drunen, The Netherlands) and led into a closed circuit existing of an $8 \mathrm{~m}$ long tube, $60 \times 60 \mathrm{~cm}$ wide. Inside the closed circuit (the shunt), preheated air passed over an ultrasonic humidifier (Stulz Gmbh, Hamburg, Germany). As a result of adiabatic cooling, the desired temperature was reached and relative humidity increased towards saturation. At the end of the shunt the saturated air was mixed with the filtered outdoor air. Within the cooling or heating section (Bo-air, Drunen, The Netherlands) the air was tuned to achieve the desired conditions. The air output section consisted of a diaphragm valve to ensure a constant flow towards the wind tunnel and a manual air valve for air inlet to the duct with the pre-heating section. The air treatment system was computer-controlled using EXOcompact technology (RT2000 B.V., Waardenburg, The Netherlands. All experiments were conducted with air of $27 \pm 1{ }^{\circ} \mathrm{C}$ and a relative humidity of $70 \pm 3 \%$. The wind speed in the arena was $200 \pm 10 \mathrm{~mm} / \mathrm{s}$.

\section{Wind tunnel}

The side walls and floor of the arena were constructed of black recycled polycarbonate and the ceiling was made of transparent Lexan polycarbonate (WSV Kunststoffen, Utrecht, The Netherlands) (Figure 2, main document). A plastic container (diameter $5 \mathrm{~cm}$, height $3 \mathrm{~cm}$ ) holding a single female mosquito was placed at the downwind end of the flight arena, exposing the mosquito to the airstream when the lid was removed. The bottom of the container was made of meshed metal gauze to allow air to pass through. Flight images were recorded using the Noldus MPEG Recorder 1.0 software (Noldus Information Technology, Wageningen, The Netherlands) that digitized the images from the cameras using an encoder board installed on the PC. Four Tracksys (Nottingham, UK) infrared light units were placed at the front of the wind tunnel, facing the air flow. Each unit contained an array of 90 infrared LEDs 


\section{Chapter 4}

emitting light with peak output at a wavelength $\lambda$ of $880 \mathrm{~nm}$. To optimize lighting conditions, four IR lights containing 168 LEDs $(\lambda>920 \mathrm{~nm}$ ) each (Reinaert Electronics, Amsterdam, The Netherlands) were added in the same line as the Tracksys IR lights. The reflection of IR light from the mosquitoes' wings was filmed with two Cohu 47222000/0000 monochrome CCD video cameras (Cohu, San Diego, CA,USA) with Fuji non-tv f1.4 $9 \mathrm{~mm}$ lenses, synchronized within $0.01 \mathrm{~s}$. The cameras were mounted at 120 and $110 \mathrm{~cm}$ from the top of the wind tunnel, respectively, with their optical axes at an angle of 40 degrees. This solution minimized the reflections on the transparent top wall of the wind tunnel. To enable spatial orientation of the mosquito, low light conditions were provided by two incandescent lights ( $7 \& 15$ Watt) facing away from the arena. Light level in the human visual spectrum was less than 0.74 Lux measured at the center top of the arena (Apogee instruments Inc., Logan, UT, U.S.A.).

\section{Temperature measurements}

At completion of the experiments, the effect of the heat element on the air temperature on the upwind side of the screen was measured using a TC-08 thermocouple data logger (Pico Technology Ltd, St Neots, U.K.) connected to five type $\mathrm{K}$ thermocouples. During measurement series 1 and 2 , the thermocouples were placed in a horizontal line with the heat source, starting at the upwind screen of the flight arena. To demonstrate the effect of convection, one series of readings was conducted in the positive $z$ direction under an angle of 13 degrees, with intervals of 5 $\mathrm{cm}$, measured from the top of the heat source. The angle of 13 degrees was chosen as a result of plume simulations using the Safex® fog generator. After calibration of the thermocouples, the mean of 60 readings $(1 \mathrm{~Hz})$ was calculated for each thermocouple (Figure S1). 


\section{A 3D analysis of mosquito flight}

\section{S2: Computational methods to produce 3-D track data}

For a precise 3-D reconstruction of a mosquito flight, Track3D required input for lens correction, calibration, filtering and interpolation. Track3D accommodated the air velocity and odour plume as created for the experiments; it produced 3-D target coordinates, accuracy checks for calibration and a 3-D reconstruction. Flight parameters (definitions in Table S1) could be calculated and presented per mosquito track in Microsoft Excel (Microsoft Office Professional edition, 2003) output files. The target path was reconstructed in a 3-D graph using different markers for positions inside or outside the plume (Figure 3, main document).

To reduce noise, the 2-D coordinates of tracked mosquitoes were smoothed by a Butterworth filter in combination with the zero phase shift routine filtfilt from Matlab 7.0 (Mathworks) with settings 'filter order 2' and cut-off frequency $8 \mathrm{~Hz}$. Missing values, if not more than four in a row, were filled in by interpolated values. To this end, third order spline functions were used for all coordinates in a coherent block of data. Ideally, rays from camera centres to target projections on images should intersect at the 3-D target position. In reality there will be a distance between the rays, called the intersection error. The error was expressed in pixels, and only the smaller of the two values (one value for each camera, because of different scales) was used. 2-D coordinates which deviated more than 67 pixels from neighbouring coordinates were deleted. This removes most artefacts such as reflections that were taken as target. The threshold of 67 pixels would theoretically result in a flight speed of $>200 \mathrm{~cm} / \mathrm{s}$ relative to the walls with the assumption that there is no change in $z$ direction.

Lens correction was applied by taking single pictures of a checkerboard in 20 different positions and using Matlab software from http://www.vision.caltech.edu/bouguetj/ calib_doc/index.html to find for each camera the parameters PP (principal point), FL (focal length), and $k_{c}$ (coefficients of $r^{2}$ and $r^{4}$ for radial distortion where $r$ is the distance from the centre of the image). The relationship between normalized distorted coordinates $x_{d}$ and normalized corrected coordinates $x_{n}$ is $x_{d}=x_{n}\left(1+k_{c 1} \cdot r^{2}+k_{c 2} \cdot r^{4}\right)$, with $r^{2}=x_{n}^{2}\left(x_{d}, x_{n}\right.$ and $x_{p}$ are 2D vectors). Where the reverse relationship was needed, the corresponding parameters $k_{c i l}$ and $k_{c i 2}$ were numerically approximated. $x_{n}$ follows from the pixel coordinates $x_{p}$ as:

$\boldsymbol{x}_{d}=\left(x_{p}-P P\right) / F L$

$r^{2}=\boldsymbol{x}_{d}^{2}$

$\boldsymbol{x}_{n}=\boldsymbol{x}_{d}\left(1+k_{c i 1} \cdot r^{2}+k_{c i 2} \cdot r^{4}\right)$. 


\section{Chapter 4}

After positioning of the cameras above the wind tunnel a calibration object of $60 \mathrm{x}$ $58.5 \times 57.5 \mathrm{~cm}$ was placed inside the flight arena. The object, made of black epoxy aluminium, had 28 white markers of circular shape $(\varnothing=15 \mathrm{~mm})$ distributed in two levels and with known 3-D coordinates. Markers on each calibration image (for each camera view) were indicated in a fixed order, after which Track3D was used to determine the marker centroids. From the sets of 2-D and known 3-D coordinates, DLT parameters were calculated as defined by

$$
\begin{aligned}
& u=\left(L_{T} x+L_{2} y+L_{3} z+L_{4}\right) /\left(L_{9} x+L_{l y} y+L_{I I} z+1\right) \\
& v=\left(L_{s} x+L_{0} y+L_{z} z+L_{d}\right) /\left(L_{9} x+L_{10} y+L_{11} z+1\right) \text {, }
\end{aligned}
$$

where $u$ and $v$ are the 2-D, and $x, y, z$ are the 3-D coordinates. The 11 DLT parameters follow by application of standard linear algebra (de Groot \& van Leeuwen, 2002, and references therein). Each camera had its own set of parameters.

The calibration results were checked in two ways. First, the known 3-D coordinates were combined with the DLT parameters to calculate the expected 2-D marker coordinates. These were compared with the measured camera coordinates. Second, the 3-D coordinates of the calibration markers were calculated from the measured 2-D marker coordinates and the DLT parameters. They were compared with the known 3-D coordinates. The calibration accuracy of the set-up was $0.5 \%$ of the dimensions of the tracking arena. 


\section{A 3D analysis of mosquito flight}

Table S1. Movement parameters calculated by Track3D. For the complete list with information about the latest version of Track3D and associated video recording and tracking software, see www.noldus.com/track3d.

\begin{tabular}{|c|c|c|}
\hline Name & Description/Formula & Example \\
\hline$x$ & $\begin{array}{l}\text { The } X \text { coordinates in } \mathrm{mm} \text { of the supposed centre of the } \\
\text { tracked animal. The axis origin }(0,0,0) \text { is the point chosen } \\
\text { as origin during calibration }\end{array}$ & \\
\hline $\mathrm{Y}$ & $\begin{array}{l}\text { The Y coordinates in mm of the supposed centre of the } \\
\text { tracked animal. The axis origin }(0,0,0) \text { is the point chosen } \\
\text { as origin during calibration }\end{array}$ & \\
\hline Z & $\begin{array}{l}\text { The } Z \text { coordinates in mm of the supposed centre of the } \\
\text { tracked animal. The axis origin }(0,0,0) \text { is the point chosen } \\
\text { as origin during calibration }\end{array}$ & \\
\hline Plume code & $\begin{array}{l}\text { Indicates where the animal's centre is located relative to } \\
\text { the supposed plume cone: } \\
1 \text { - Within the cone } \\
2-\text { Within the buffer zone } \\
3 \text { - Outside the cone and buffer zone }\end{array}$ & \\
\hline Path 3d & $\begin{array}{l}\text { The distance moved from the starting point of the present } \\
\text { block } 1 \text { up to the current sample } 2 \text {. It is measured as the } \\
\text { sum of the segments joining adjacent samples and is } \\
\text { expressed in mm. }\end{array}$ & $\begin{array}{l}\text { Example of the path 3d parameter. Dots are } \\
\text { sample points in the 3D space. The sample } s_{0} \text { is } \\
\text { the start of the block. Path } 3 d_{\text {equals }} d_{1} \text { for } \\
\text { sample } s_{\gamma^{\prime}} d_{1}+d_{2} \text { for sample } s_{z^{\prime}} d_{1}+d_{2}+d_{3} \text { for } \\
\text { sample } s_{3^{\prime}} \text { etc. }\end{array}$ \\
\hline Speed & $\begin{array}{l}\text { The scalar, absolute value of the velocity vector in the 3D } \\
\text { space. The velocity vector is calculated for each sample } \\
\text { sk with one of the three formulas, according to how many } \\
\text { samples are present around it. } \\
\text { - When only one sample sk-1 occurs before sk: Velocity } v_{k} \\
=\left(p_{k}-p_{k-1}\right) /\left(t_{k}-t_{k-1}\right) \\
\text { - When one sample sk-1 occurs before and another } s_{k+1} \\
\text { occurs after } s_{k} \text { : Velocity vk }=\left(p_{k+1}-p_{k-1}\right) /\left(t_{k+1}-t_{k-1}\right) \\
\text { - When more samples are available around sk (as in most } \\
\text { cases): Velocity is the value of the derivative of a cubic } \\
\text { spline function through all samples of that block, at } \\
\text { sample } s_{k} \text {. } \\
\text { The position vector p contains the } X, Y, Z \text { coordinates and } t \\
\text { is the time of samples. Speed is expressed in } \mathrm{mm} / \mathrm{s} \text {, and } \\
\text { can only have positive values. }\end{array}$ & $\begin{array}{l}\text { Example of the speed parameter. Speed is the } \\
\text { absolute value of the velocity vector } v_{k} \\
\text { calculated for each sample } s_{k} \text {. }\end{array}$ \\
\hline
\end{tabular}




\section{Chapter 4}

\begin{tabular}{|c|c|c|}
\hline Name & Description/Formula & Example \\
\hline Ang. ch 3d & $\begin{array}{l}\text { Stands for Angular change } 3 \mathrm{~d} \text {. It is calculated as the } \\
\text { angle in the } 3 \mathrm{D} \text { space formed by the segment joining } \\
\mathrm{sk} \text { and } \mathrm{s}_{\mathrm{k+1}} \text { and the prolongation of the segment joining } \\
\mathrm{sk} \text { and } \mathrm{s}_{\mathrm{k-1}-1} \text {. Angular change } 3 d \text { is expressed in degrees } \\
\text { and can only be positive. }\end{array}$ & $\begin{array}{l}\text { Example of the Angular change 3d parameter. } \\
\text { Angular change 3d is the angle in the 3D space } \\
\text { formed by the velocity vector and the } \\
\text { prolongation of the previous velocity vector. }\end{array}$ \\
\hline Ang. vel. 3d & $\begin{array}{l}\text { Stands for Angular velocity } 3 d \text {. It is the angular change } \\
3 d \text { per time unit. It is expressed in degrees } / \mathrm{s} \text {. }\end{array}$ & \\
\hline
\end{tabular}

1 Uninterrupted series of valid $X, Y, Z$ data points

2 Set of $X, Y, Z$ coordinates associated with a particular time value. Corresponds to a pair of $X, Y$ coordinates in 2- $D$ video tracking performed by EthoVision.

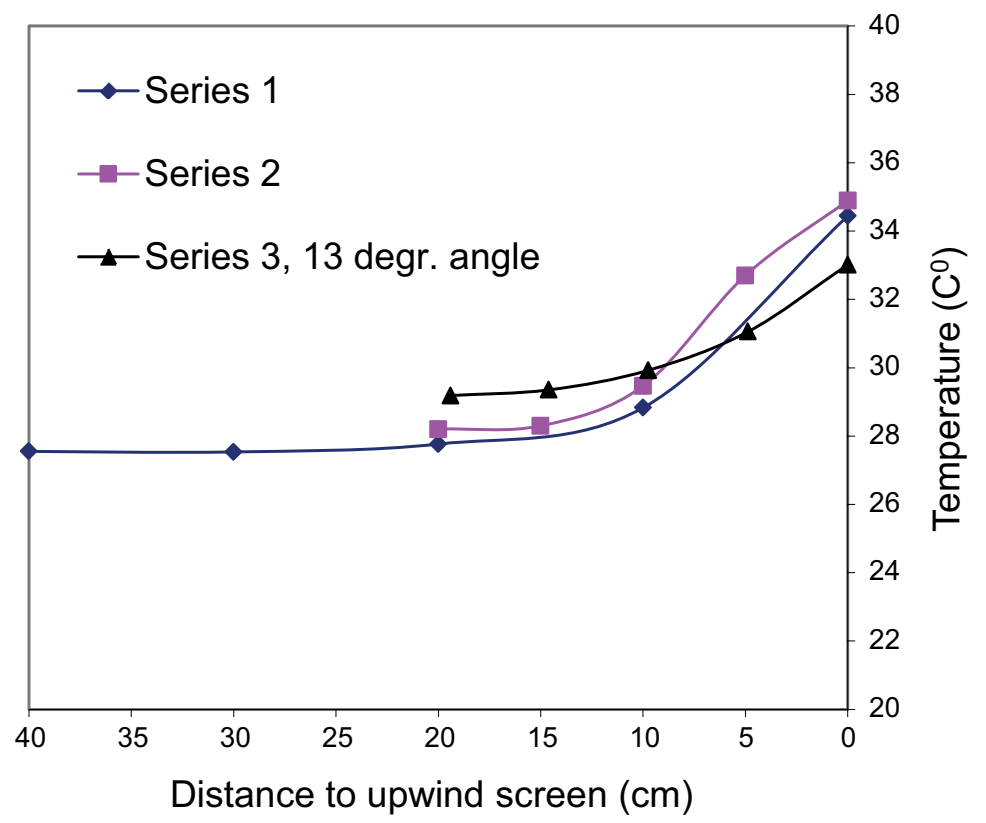

Figure S1. Three series of temperature readings measured inside the flight arena. During series 1 and 2, the thermocouples were placed in a horizontal line with the heat source, starting at the upwind screen. Series 3 was measured in positive $z$ direction under an angle of 13 degrees. 


\section{A 3D analysis of mosquito flight}

Table S2. ANOVA for main effects and interaction of treatments on the tangent (crosswindbehaviour) for different distances to the upwind screen. The mean ( \pm s.e.m) tangent is given per section followed by main effect and interaction estimates for each treatment. Results are given for the $x y$ - and $x z$ plane respectively and indicated with * for different levels of significance. Signif. codes: <

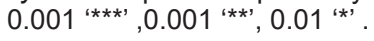

\begin{tabular}{|l|c|c|c|c|c|}
\hline$x y$ & Section I IV & Section I & Section II & Section III & Section IV \\
& $0-60 \mathrm{~cm}$ & $0-15 \mathrm{~cm}$ & $15-30 \mathrm{~cm}$ & $30-45 \mathrm{~cm}$ & $45-60 \mathrm{~cm}$ \\
\hline Mean (s.e.m.) tangent & 0.862 & 1.051 & 0.696 & 0.625 & 0.567 \\
& $(0.024)$ & $(0.026)$ & $(0.037)$ & $(0.039)$ & $(0.037)$ \\
\hline Odour main effect & $0.088^{* * *}$ & $0.057^{*}$ & $0.118^{* *}$ & $0.116^{* *}$ & $0.133^{* * *}$ \\
\hline Heat main effect & $0.061^{*}$ & $0.073^{* *}$ & 0.006 & 0.074 & 0.057 \\
\hline Odour.heat interaction & -0.003 & -0.027 & 0.050 & 0.038 & 0.034 \\
\hline$x z$ & Section I - IV & Section I & Section II & Section III & Section IV \\
\hline Mean (s.e.m.) tangent & 1.311 & 1.677 & 0.903 & 0.789 & 0.777 \\
\hline Odour.heat interaction & -0.040 & -0.030 & -0.025 & 0.005 & 0.021 \\
\hline Heat main effect & $(0.037)$ & $(0.041)$ & $(0.044)$ & $(0.050)$ & $(0.048)$ \\
\hline
\end{tabular}




\section{Chapter 4}
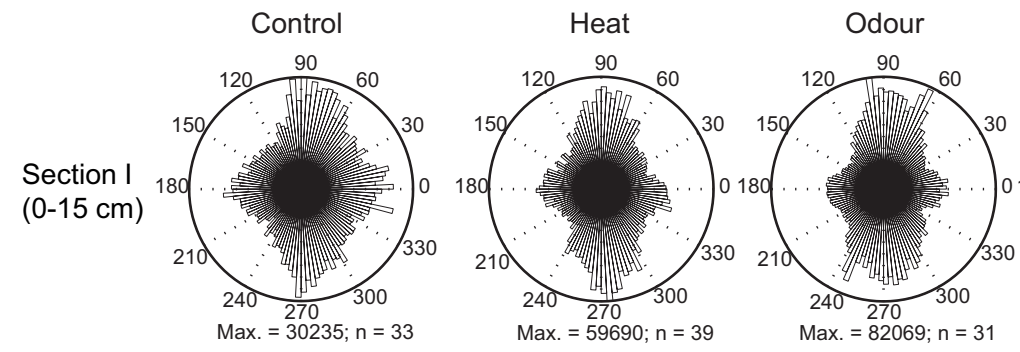

\section{Odour and heat}
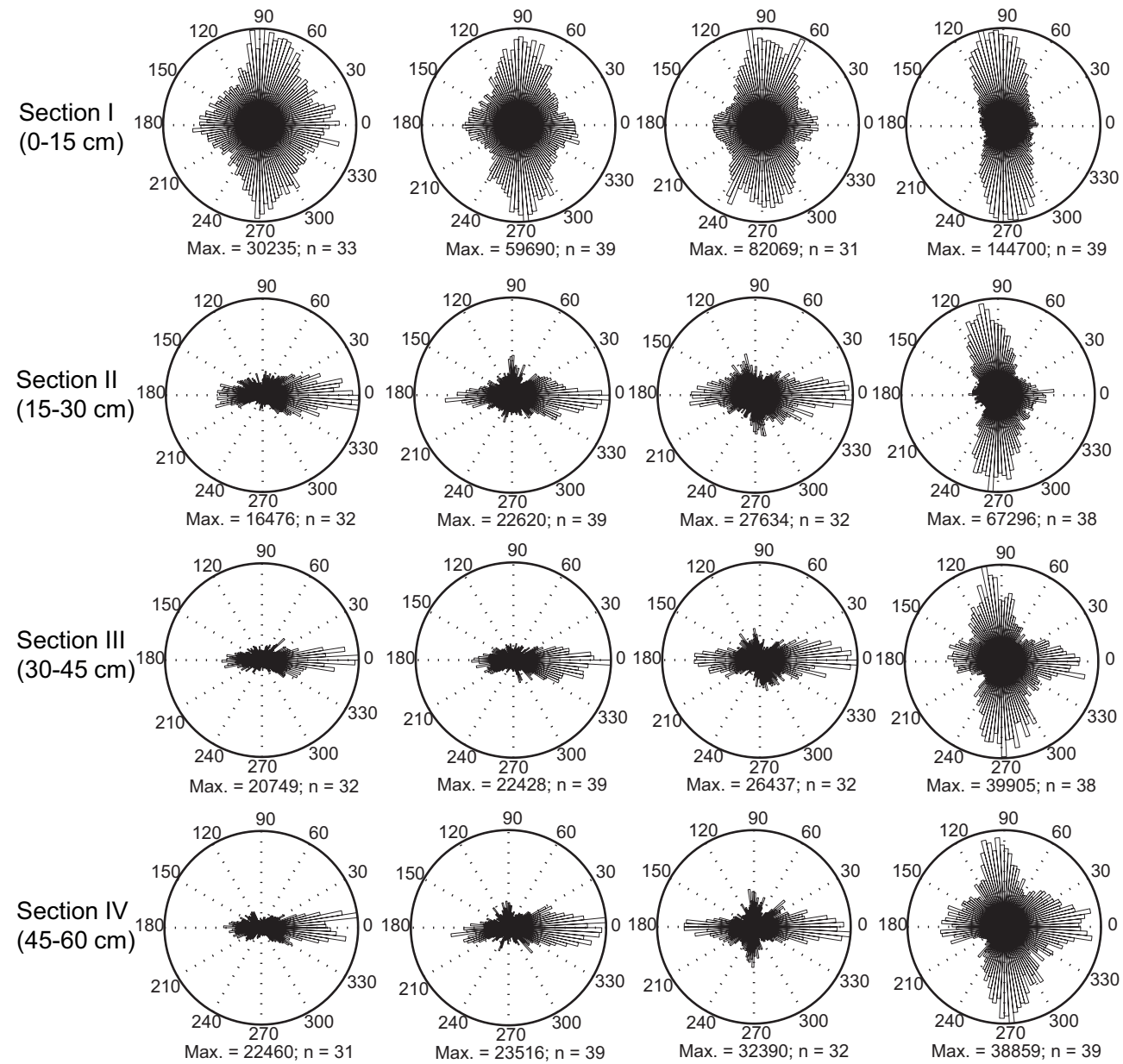

Figure S2. Rose-diagrams with distributions of the sum of velocities per $3^{\circ}$ bins in the horizontal (xy) plane. The sum of velocities is a measure of the total distance moved within the defined direction. The max. value is a measure of scale and represents the maximum sum of velocities plotted within the diagram. For each treatment distributions are plotted for four different sections from the upwind screen where ' 0 ' represents upwind flight. 


\section{A 3D analysis of mosquito flight}

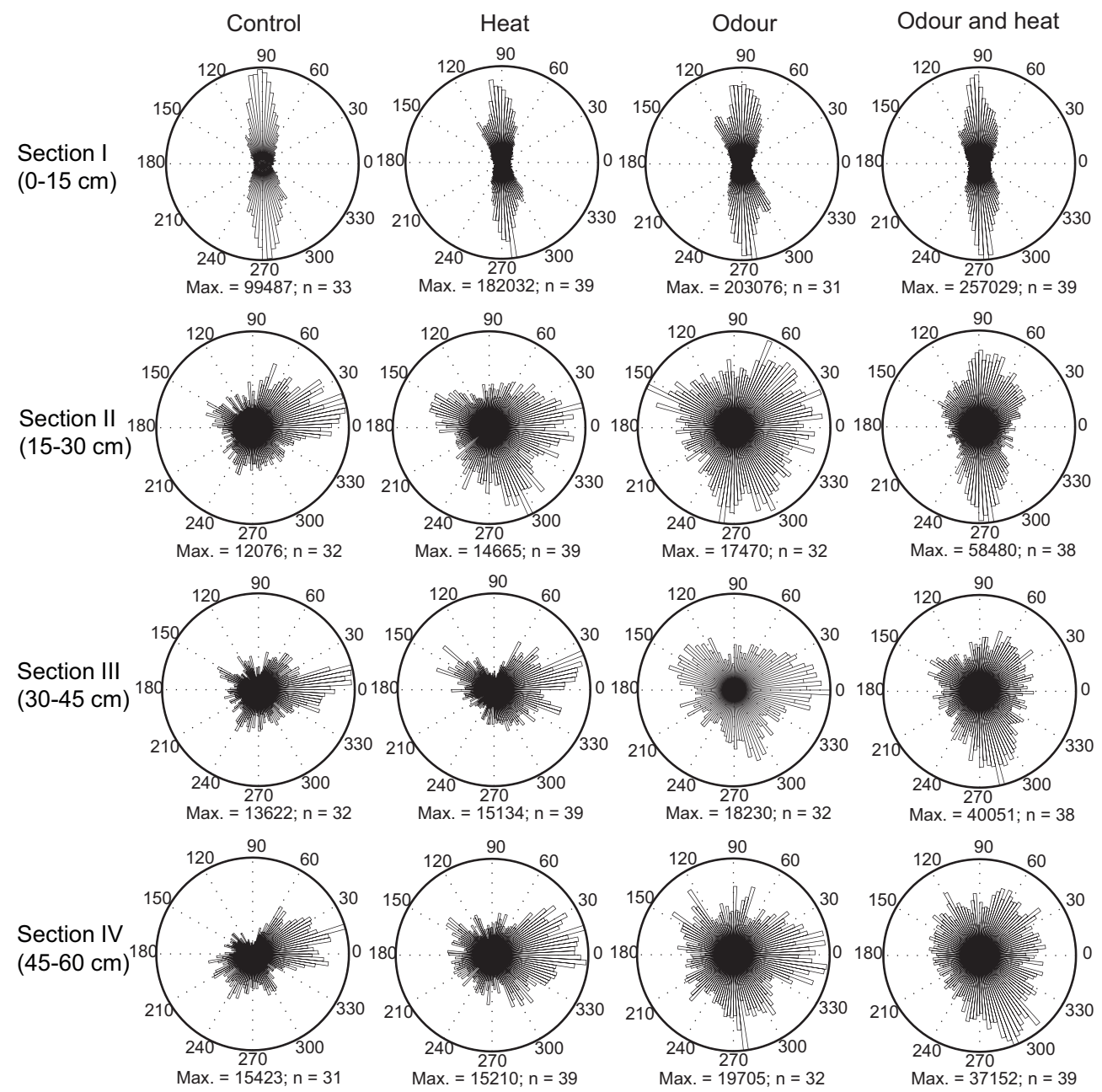

Figure S3. Rose-diagrams with distributions of the sum of velocities per $3^{\circ}$ bins in the vertical $(x z)$ plane. The sum of velocities is a measure of the total distance moved within the defined direction. The max. value is a measure of scale and represents the maximum sum of velocities plotted within the diagram. For each treatment distributions are plotted for four different sections from the upwind screen where ' 0 ' represents upwind flight. 


\section{Chapter 4}

Table S3. The mean difference of ' $x$ in' - ' $x$ out' presented for each mosquito while entering the plume and the mean difference of ' $x$ out' $-x$ in' upon exiting. A positive mean represents upwind progress. $n$ Represents the number of occurrences within the recorded track.

\begin{tabular}{|l|l|l|l|l|l|l|}
\hline & \multicolumn{3}{|c|}{$\begin{array}{c}\text { Entering } \\
\text { Difference 'x in'- 'x out' }\end{array}$} & \multicolumn{3}{c|}{$\begin{array}{c}\text { Exiting } \\
\text { Difference 'x out'- 'x in' }\end{array}$} \\
\hline Mosquito & \multicolumn{2}{|c|}{ Mean } & & & Mean & \\
ID & $\mathrm{n}$ & $(\mathrm{mm})$ & s.e.m. & $\mathrm{n}$ & $(\mathrm{mm})$ & s.e.m. \\
\hline 42 & 131 & 27.08 & 6.07 & 112 & -6.49 & 7.98 \\
\hline 43 & 19 & 29.53 & 24.71 & 14 & 2.57 & 5.52 \\
\hline 44 & 71 & 21.76 & 9.99 & 70 & -21.79 & 10.92 \\
\hline 52 & 4 & 8.50 & 8.39 & 3 & 13.00 & 5.29 \\
\hline 57 & 46 & 31.87 & 13.86 & 36 & -16.08 & 8.20 \\
\hline 60 & 6 & 92.33 & 93.96 & 6 & -14.00 & 22.00 \\
\hline 62 & 4 & 123.50 & 171.25 & 2 & 21.00 & 26.00 \\
\hline 64 & 16 & 35.56 & 20.81 & 14 & -8.79 & 3.52 \\
\hline 65 & 33 & 31.15 & 18.38 & 29 & -2.31 & 6.78 \\
\hline 66 & 11 & 81.09 & 62.87 & 11 & -38.64 & 25.69 \\
\hline 67 & 9 & 25.67 & 19.44 & 8 & 60.38 & 28.85 \\
\hline 69 & 114 & 37.17 & 7.40 & 83 & 0.94 & 5.31 \\
\hline 70 & 17 & 5.71 & 7.76 & 17 & 4.71 & 3.27 \\
\hline 78 & 48 & 9.44 & 16.04 & 31 & -6.90 & 4.85 \\
\hline 79 & 35 & 19.69 & 7.74 & 22 & 11.36 & 4.42 \\
\hline 81 & 26 & -9.77 & 10.06 & 17 & -65.53 & 51.92 \\
\hline Total & 590 & 27.36 & 3.75 & 475 & -8.11 & 3.49 \\
\hline
\end{tabular}
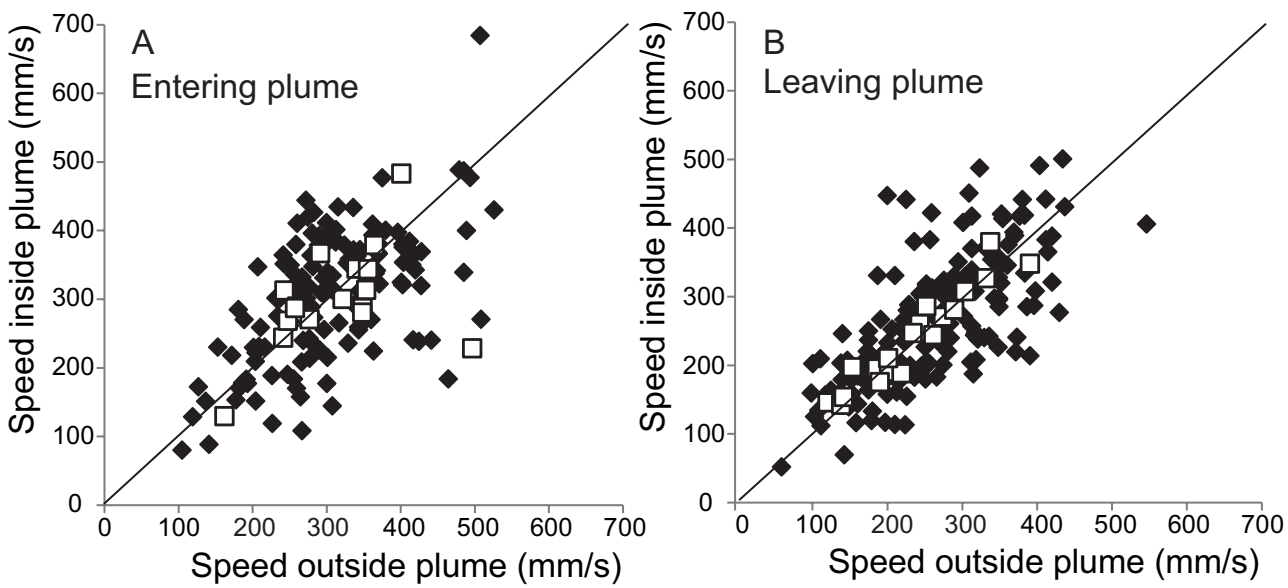

Figure S4. Change in flight speed upon entering or leaving the plume. A - The mean speed of mosquitoes exposed to odour + heat of 10 frames before entering the plume and the subsequent first 10 frames while in the plume after crossing the buffer zone of $25 \mathrm{~mm}(n=17)$. B - Represents the mean speed of 10 frames before exiting the plume and the first 10 frames after leaving the plume $(n=19)$. The solid line represents a theoretical situation where mean speeds of entering/leaving are equal. The open squares show the average value of all entering/leaving occurrences per individual. 



$$
\text { Nî }
$$




\section{Chapter 5}

\section{Visualization of house-entry behaviour of malaria mosquitoes}

Jeroen Spitzen,

Teun Koelewijn,

W. Richard Mukabana,

Willem Takken

Published in Malaria Journal 15:233 (2016). 
Malaria mosquitoes often blood feed indoors on human hosts. The mosquitoes predominantly enter houses via open eaves. Host-seeking is odour-driven, and finding a host depends on the quality of the odour plume and whether the route towards the host is free of obstructions. Little is known about in-flight behaviour of mosquitoes during house entry. This semi-field study visualizes mosquito house entry in three dimensions (3D) and offers new insights for optimizing vector control interventions. The approach and house entry of Anopheles gambiae sensu stricto was studied in a semi-field set-up using video-recorded flight tracks and 3D analysis. Behavioural parameters of host-seeking female mosquitoes were visualized with respect to their position relative to the eave as well as whether a mosquito would enter or not. Host odour was standardized using an attractive synthetic blend in addition to $\mathrm{CO}_{2}$. The study was conducted in western Kenya at the Thomas Odhiambo Campus of the International Centre of Insect Physiology and Ecology, Mbita. The majority of host-seeking An. gambiae approached a house with a flight altitude at eave level, arriving within a horizontal arc of $180^{\circ}$. Fifty-five per cent of mosquitoes approaching a house did not enter or made multiple attempts before passing through the eave. During approach, mosquitoes greatly reduced their speed and the flight paths became more convoluted. As a result, mosquitoes that passed through the eave spent more than $80 \%$ of the observed time within 30 $\mathrm{cm}$ of the eave. Mosquitoes that exited the eave departed at eave level and followed the edge of the roof $(12.5 \%)$ or quickly re-entered after exiting (9.6\%). The study shows that host-seeking mosquitoes, when entering a house, approach the eave in a wide angle to the house at eave level. Less than $25 \%$ of approaching mosquitoes entered the house without interruption, whereas $12.5 \%$ of mosquitoes that had entered left the house again within the time of observation. Advances in tracking techniques open a new array of questions that can now be answered to improve household interventions that combat malaria transmission. 


\section{Visualization of house-entry behaviour}

\section{Introduction}

The anthropophilic and historically endophilic malaria vector Anopheles gambiae s.s. is known to exploit host odours to find a suitable blood meal for egg production (Cardé \& Gibson, 2010; Costantini et al., 1999; Takken \& Knols, 1999). In a field setting, this would mean that after emergence as adults, initial nectar feeding and joining mating swarms, the mosquitoes enter into a host-seeking state which will commonly lead them to the vicinity of or into human dwellings (Gillies, 1955; Haddow, 1942).

Navigation of the mosquitoes towards inhabited houses is initiated by increases in $\mathrm{CO}_{2}$ levels in combination with a number of host odours (Cardé \& Willis, 2008; Loon et al., 2015; Mukabana et al., 2010). Odour plumes become wider and more diluted at greater distance from the source (Koehl, 2006). The turbulent forces of diffusion in wind stretches and stirs odour filaments, creating gaps of odour-free air in a plume while it expands and is transported with the wind (Murlis et al., 1992). Filaments are stirred around in eddies (ranging from millimetres to hundreds of metres) and intermingled with the surrounding odour-free air (Koehl, 2006). These turbulent forces in air cause a spatially complex and temporally changing three-dimensional distribution of odour concentrations in natural habitats (Koehl, 2006). Many insects exhibit behaviour that enables them to follow odour plumes to the source (Murlis et al., 1992), however, there is much diversity in how insects navigate along such plumes to reach their target. For mosquitoes, one can expect differences between diurnal and nocturnal species and the multimodal use of visual cues or the relative importance of a general host cue such as $\mathrm{CO}_{2}$ (Cardé \& Gibson, 2010; McMeniman et al., 2014).

\section{House entry}

Studies on mosquito house entry often describe the approach and entry of indoorbiting mosquitoes based on catches with traps that can intercept house entry or exit behaviours (Gillies, 1955; Grieco et al., 2000; Ogoma et al., 2010). These studies are based on the knowledge that anopheline mosquitoes predominantly enter and exit via eaves (Oxborough et al., 2015; Snow, 1987). An extensive field study on An. gambiae on house entry is well described by Snow (1987), who elaborates on earlier work in The Gambia reviewed by Gillies (1980), in which the long-range orientation to human dwellings is attributed to the dispersion of increased $\mathrm{CO}_{2}$ levels. More recent work has looked at this topic from the point of house entry and has begun to unravel the role of specific host cues once mosquitoes are inside a house (Webster et al., 2015). 


\section{Chapter 5}

Grieco et al. (2000) observed that doors, windows and eaves are large portals of house entry for An. vestitipennis. Host-seeking mosquitoes find little or no obstacle to entering a house when one of these openings is available (Lindsay \& Snow, 1988). Walls form obstacles, but some mosquitoes will still be able to enter a house if there are gaps in the wall ranging from 1-2 cm wide (Grieco et al., 2000; Lindsay \& Snow, 1988). Reviewing mosquito house entry, Lindsay et al. (2002) concluded that houses with open eaves or houses that lack ceilings had higher numbers of mosquitoes and higher levels of malaria compared to neighbouring houses with closed eaves or ceilings. Snow suggests that the majority of mosquitoes fly below a height of $1 \mathrm{~m}$ towards a house and when they reach the house they will at some point fly upwards to eave level and then move indoors.

\section{Vector control interventions}

Innovative control strategies are needed to reduce vector-host interactions for the control of malaria (WHO-RBM, 2015). The dispersion of host cues is related to house design and this has, together with physical barriers, implications for the level of indoor biting (Kirby, 2013; Tusting et al., 2015; Wanzirah et al., 2015). Indirectly, the level of house entry also has an impact on the proportion of mosquitoes that would eventually try to feed outdoors, and may be responsible for outdoor malaria transmission (Durnez et al., 2013; Killeen, 2014). Along with the development of new tools, proper implementation is highly relevant: Jawara et al. (2009) showed that odour-baited MM-X traps set $15 \mathrm{~cm}$ from the ground at 2 and $10 \mathrm{~m}$ from a house caught more mosquitoes than traps set at $150 \mathrm{~cm}$ from the ground, but next to the house comparable numbers of mosquitoes were caught at 15 and at 150 $\mathrm{cm}$ in height. The efficiency of push-pull strategies and other large-scale efforts to reduce parasite transmission can benefit from detailed behavioural descriptions on how repellents and attractants function spatially (Hiscox et al., 2014; Menger et al., 2015).

Advances in tracking techniques can provide more detail on flight behaviours without artificial obstacles, such as interception traps, which are likely to affect initial flight paths (Butail et al., 2012; McMeniman et al., 2014; Spitzen et al., 2013). The data provide new insights on how to disrupt mosquitoes' natural behaviour or cause interference along their route (Menger et al., 2015; Mnyone et al., 2012; Parker et al., 2015; Sutcliffe \& Colborn, 2015; Tusting et al., 2015; Wanzirah et al., 2015). The present study describes the house entry and exit behaviour of mosquitoes that were lured towards a house by standardized human host cues in a semi-field setting. Three-dimensional (3D) reconstructions of recorded flight tracks were made, in which mosquitoes were observed to enter, exit or move away from the eave of an 


\section{Visualization of house-entry behaviour}

experimental house. By providing knowledge on flight behaviour of mosquitoes insitu, improvements of trapping systems, push-pull systems and netting/screening designs can be made.

\section{Methods}

Mosquitoes

Anopheles gambiae sensu stricto (Mbita strain) mosquitoes were obtained from the insectaries located at the Thomas Odhiambo Campus of the International Centre of Insect Physiology and Ecology (icipe-TOC) in Mbita Point township, western Kenya $\left(00^{\circ} 25^{\prime} S, 34^{\circ} 13^{\prime} \mathrm{E}\right)$. The Mbita strain has been reared since 2001 under ambient climatic conditions. Eggs were laid on wet filter paper and placed in filtered water from Lake Victoria, in plastic trays. Aquatic stages of the mosquitoes were reared in a screen house and larvae were fed daily on cat food (Go-Cat complete Purina, Croydon, England). Pupae were collected daily and transferred to mesh-covered $(30 \times 30 \times 30 \mathrm{~cm})$ cages. Mosquitoes used for the experiment did not have access to a blood meal. Mosquitoes were fed on a 6\% glucose (Excel Chemicals Ltd., Nairobi, Kenya) solution.

\section{Experimental set-up}

Experiments were done inside a screen house, the semi-field set-up, located at icipeTOC. The screen house (flight arena) was made of wooden poles and beams and a roof of thatch covered with corrugated iron. The flight arena of the mosquitoes was $12.8 \times 4.75 \times 2.25 \mathrm{~m}$. The screen house was covered with dark-green shade netting permitting airflow (wind) to enter the system. Inside the house there was another netting to prevent the escape of any released mosquitoes. The base of the screen house consisted of a top layer of sand and pebble-sand and an under layer of rocks and compact sand. An experimental house, constructed of wood and a corrugated iron roof, was built inside the screen house. The house had two windows and a door. In many African houses there is a gap between the top of the wall and the roof, an open eave, to provide ventilation. The experimental house had a $15-\mathrm{cm}$ wide eave on two sides of the house. Instead of filming the full distance across one eave, the camera view covered the central area in front of the house and below the eave to obtain an as large as possible tracking arena with 3D coverage (Figure 1). Parts of the wall and roof were covered with black recycled polycarbonate (WSV Kunststoffen, Utrecht, The Netherlands) to optimize the contrast during filming. Inside the experimental house, there was a hook for suspending a Mosquito Magnet $\mathbb{X}$ (MM-X) odour-baited trap and for a bed net. The release point of the mosquitoes was at $4.25 \mathrm{~m}$ in front of the experimental house. Attached to the screen house, a control room of $5.40 \times 1.80 \mathrm{~m}$ was installed where a computer and all other operating 


\section{Chapter 5}

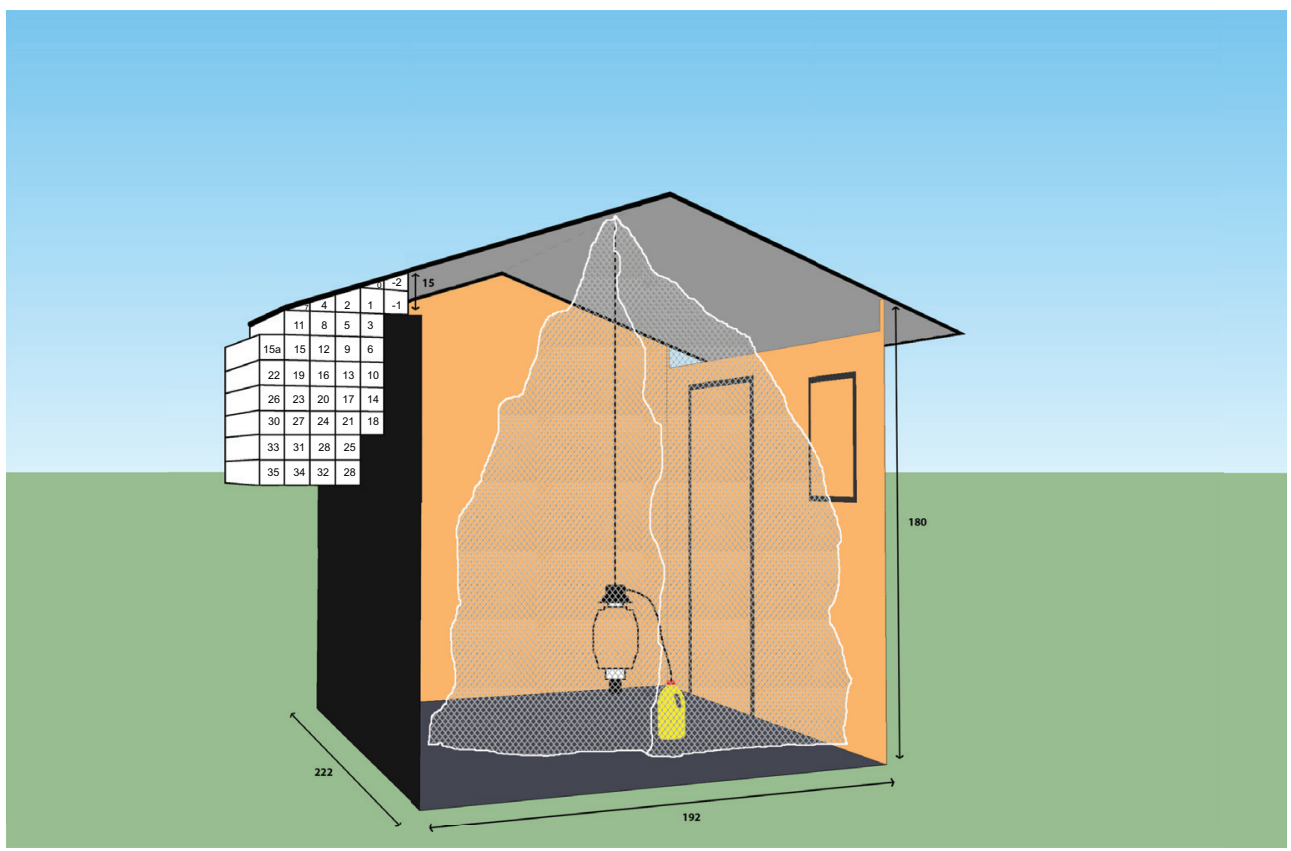

Figure 1: Experimental house with tracked area superimposed. Measurements of the house and eave in $\mathrm{cm}$ with block-shaped zones of $70 \times 10 \times 10 \mathrm{~cm}$ for which 3D-analysis of flight parameters were performed. An untreated net was hung over an odour-baited MM-X trap during the course of filming.

systems were placed. There were no persons around in the screen house or the control room during the recording phase.

\section{Recording equipment}

Two Cohu 4722-2000/0000 monochrome CCD camera (Cohu, San Diego, CA, USA) equipped with Fuji non-tv $9 \mathrm{~mm} / \mathrm{f} 1.4$ lenses were installed on a solid frame dug into the sand at $35 \mathrm{~cm}$ from the house facing the eave. Four Tracksys (Nottingham, UK) infrared light units were placed just behind the cameras, each unit contained an array of 90 infrared LEDs emitting light with peak output at $880 \mathrm{~nm}$. The equipment was powered via an uninterruptible power supply (UPS) and computer controlled in the control room. Details on the automated tracking system and $3 \mathrm{D}$ reconstruction are based on the description given by Spitzen et al., (2013). A lightweight calibration frame was constructed of pieces of Cochranes Orbit molecular building system (Cochranes of Oxford Ltd, UK) containing 71 markers. The calibration accuracy of the set-up was $0.23 \%$ of the dimensions of the tracking arena.

\section{Experimental procedures}

The MM-X trap was used to dispense synthetic host odours that lured mosquitoes into the experimental house and to remove experimental mosquitoes after the 


\section{Visualization of house-entry behaviour}

behavioural recordings had taken place. An untreated bed net (100\% polyester, Top 2 , Kenya) was hung over the trap during the flight experiment. The composition, production and dispersion of the five-component blend and $\mathrm{CO}_{2}$ is described in detail by (Menger et al., 2014b) and (Smallegange et al., 2010b) with the exception of tetradecanoic acid (C14) that was used in a $0.0025 \mathrm{~g} / \mathrm{L}$ concentration. Female mosquitoes were three to eight days old when tested and were aspirated from the adult cages eight to ten $\mathrm{h}$ before the start of the experiment. They were placed in plastic cups of $1 \mathrm{~L}$ (PCL Mombasa, Kenya) in groups of 200 covered with mosquito gauze and provided with a paper towel soaked in water.

The trap started running at $20.00 \mathrm{~h}$, from the moment mosquitoes were released. Mosquitoes were released remotely, by lifting the lid of the mosquito container using a fishing line. At $00.00 \mathrm{~h}$, the cameras were stopped and the trap was uncovered from the bed net allowing trap entry and mosquito removal from the experimental cage. At 10.30, the MMX-trap was stopped and placed in a freezer. After 15 min the blend was removed from the trap and stored in a refrigerator at $4^{\circ} \mathrm{C}$. Caught mosquitoes were counted.

Data processing and analyses

$3 \mathrm{D}$ output data were filtered for mosquitoes with a minimum of six recorded frames $(0.24 \mathrm{~s})$ while flying. A filter for flying was set at $>27 \mathrm{~mm} / \mathrm{sec}$, being the speed of the fastest walking mosquito observed, and speeds above $2000 \mathrm{~mm} / \mathrm{sec}$ were left out as this removes most artefacts that were taken as target. In the rare event where >one mosquito came in view at the same time, only the mosquito that was tracked first was used for the analysis and the other(s) ignored.

Data were sub-divided into four groups depending on where a mosquito came into view of both cameras and was last seen in the tracking arena. These groups were referred to as: 1: in view, not entering eave; 2 : in view, entering eave; 3: exiting and re-entering eave; 4: exiting eave and departing. As individual mosquitoes could get out of view, it is possible that they were observed more than once in the same or different groups within the four $h$ of recording.

To analyse the spatial pattern of flight behaviours, the 3D space in front of the eave was divided in block-shaped zones of $70 \times 10 \times 10 \mathrm{~cm}$ (see Figure 1). The percentage of time flying per zone was first calculated for each mosquito and averaged over all mosquitoes per group and plotted in heat maps. Flight speed was defined as the magnitude of the velocity vector in space and expressed in $\mathrm{mm} / \mathrm{sec}$. The angular change of heading was defined as the change of track angle for one time step, 


\section{Chapter 5}

expressed in degrees. These two parameters were also averaged per zone block, with a minimum of six different contributing tracks per zone.

The data were not normally distributed. Differences between groups were tested for their significance using Kruskal Wallis tests via SPSS statistics version 22 (IBM Corp, USA) followed by pair-wise multiple comparisons. Adjusted p-values were calculated and considered significant at a threshold of $p<0.05$.

\section{Results}

Flight behaviour around the eave

During five experimental nights, a total of 1000 mosquitoes were released, and 473 were caught in the MM-X trap, which they could enter after the bed net was folded (from $00.00 \mathrm{~h}$ onwards). Mosquitoes could get in view of the cameras multiple times, resulting in a total of 1100 tracks that were analysed with a minimum of six frames $(0.24 \mathrm{~s})$. Of the mosquitoes that were not observed to enter via the eave $(n=604), 67.5 \%$ came first into view via zones categorized as 'sides', $19.9 \%$ from the 'front', $11.9 \%$ from 'below', and $0.7 \%$ from below/front of the house. A selection of representative video images and track reconstruction is available via Additional file 1. A heat map representing the frequency distribution of first entries per zone is presented in Figure 2.Aa. This group of tracked mosquitoes left the filmed area without entering the house in similar proportions to how they entered the arena: $72.8 \%$ from the sides, $18.2 \%$ from the front, $7.9 \%$ from below, and $1 \%$ left from below/front (Figure 2.Ab). Of the 253 mosquitoes that were observed to enter the eave (excluding individuals that exited the eave and directly re-entered), $74.7 \%$ came into view via the sides, $20.9 \%$ from the front and $4.4 \%$ were first tracked in the lowest zones (Figure 2.Ba). A large proportion (48.9\%) of the mosquitoes that exited the eave $(n=137)$ departed via the two zones just near the roof edge (Figure 2.Bb). When using the same categories as before, exiting mosquitoes were observed to depart for $68.6 \%$ via the sides, $29.2 \%$ from the front and $1.5 \%$ from below (see top view panel Figure 2.Bb).

The maximum track duration ranged from $12 \mathrm{~s}$ for a mosquito that came into view and entered via the eave to $6.7 \mathrm{~s}$ for a mosquito that exited the house and departed. Most flight tracks were much shorter and largely dependent on where a mosquito would come from or where it was flying towards. The mean track duration of mosquitoes that came into view and entered via the eave was significantly longer than that of the group that came into view but did not enter (2.23 vs $1.51 \mathrm{~s}$, Kruskal Wallis, pairwise comparison, $p<0.05$, see Table 1). Mosquitoes that exited and re-entered the house remained in close proximity to the eave and had on average a significantly 


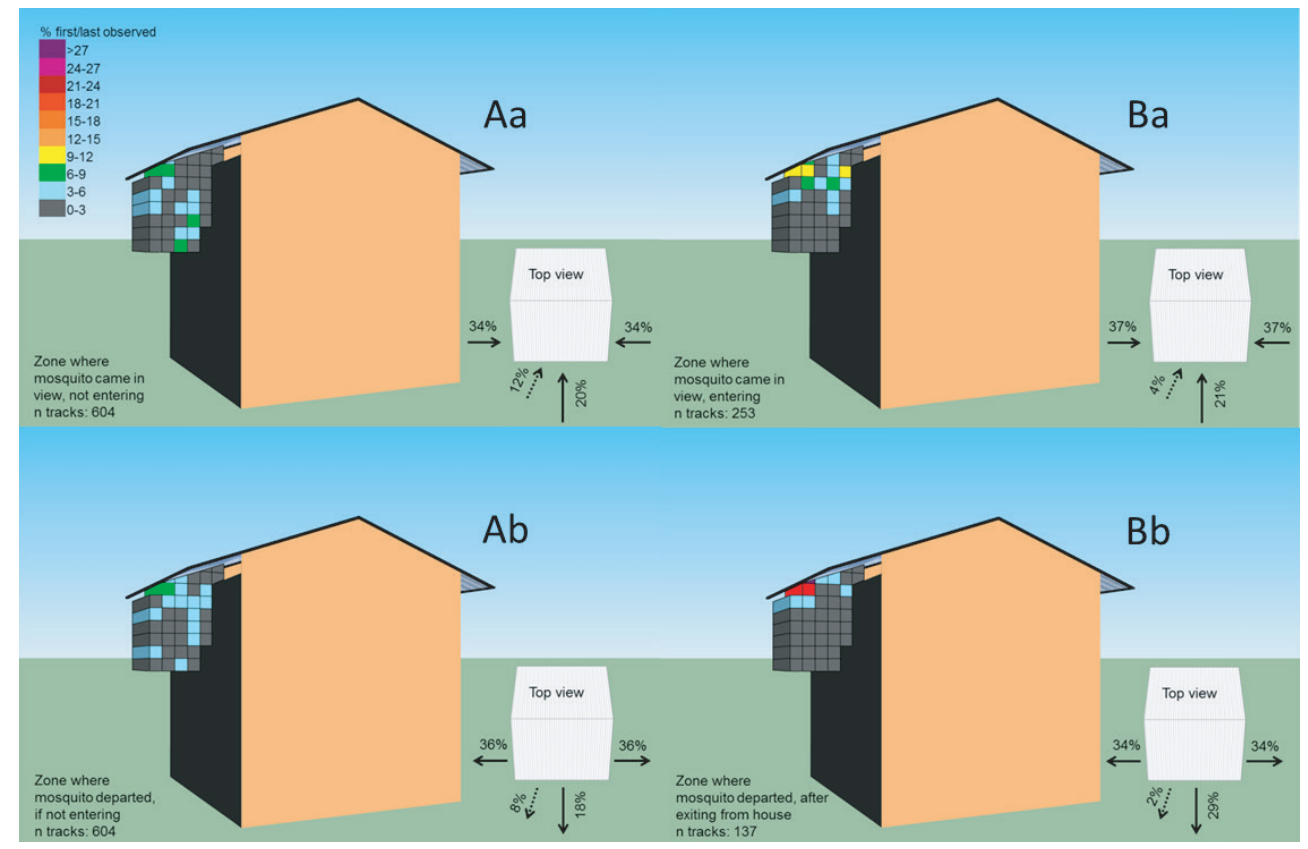

Figure 2.AB Observation arena where mosquitoes entered and left. The heat maps represent the frequency distribution in which zones mosquitoes were first or last observed within a reconstructed track. $\mathrm{Aa}$ and $\mathrm{Ab}$ represent mosquitoes that did not pass through the eave. $\mathrm{Ba}$ and $\mathrm{Bb}$ represent mosquitoes that passed through the eave. Top view images summarize the direction in which mosquitoes entered or left the area around the house.

Table 1. Mean ( \pm s.e.m.) track duration, speed and angular change of heading based on individuals with a minimum of six recorded frames $(\mathbf{0 . 2 4} \mathbf{s})$. Different letters within each column indicate significant differences in parameters depending on where mosquitoes entered/exited the filmed area (Kruskal Wallis, pairwise comparisons, adj. significance; $p<0.05)$.

\begin{tabular}{llrlll}
\hline Destination when in view & N (total 1100) & $\%$ & $\begin{array}{l}\text { Mean track } \\
\text { duration }(\mathrm{s})\end{array}$ & $\begin{array}{l}\text { Mean flight } \\
\text { speed }(\mathrm{mm} / \mathrm{sec})\end{array}$ & $\begin{array}{l}\text { Mean angle change } \\
\text { 3D (degrees) }\end{array}$ \\
\hline Did not enter house & 604 & 54.9 & $1.51 \pm 0.05 \mathrm{a}$ & $443.5 \pm 5.4 \mathrm{a}$ & $12.3 \pm 0.3 \mathrm{a}$ \\
Entered house via eave & 253 & 23.0 & $2.23 \pm 0.09 \mathrm{~b}$ & $378.8 \pm 5.6 \mathrm{~b}$ & $14.6 \pm 0.5 \mathrm{~b}$ \\
Exited house and re-entered & 106 & 9.6 & $0.89 \pm 0.10 \mathrm{c}$ & $291.2 \pm 12.0 \mathrm{c}$ & $25.5 \pm 1.6 \mathrm{c}$ \\
Exited house and departed & 137 & 12.5 & $1.75 \pm 0.09 \mathrm{~b}$ & $412.1 \pm 9.9 \mathrm{a}$ & $15.5 \pm 0.8 \mathrm{~b}$ \\
\hline
\end{tabular}

shorter track duration compared to all other groups $(0.89 \mathrm{~s}$, Kruskal Wallis, pair-wise comparison, $p<0.05$, see Table 1). Detailed information on the mean time spent in each zone at increasing distance from the eave is given in Additional file 2.

The spatial-temporal distribution of each individual flight path was calculated. The mean time spent per zone is plotted in heat maps and presented separately based 


\section{Chapter 5}

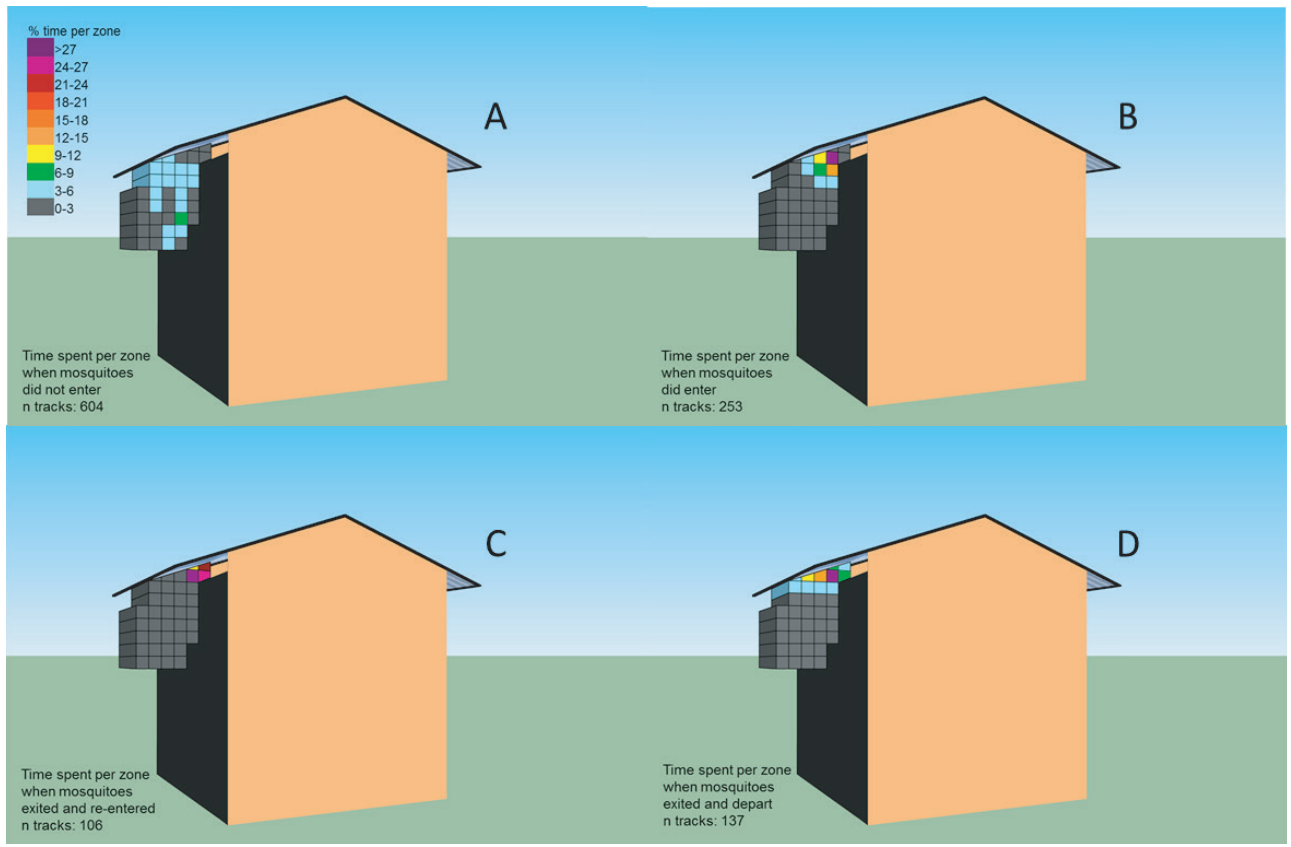

Figure 3.AD The spatial-temporal distribution per mosquito. Colours represent the percentage of time per zone for tracks in which mosquitoes were observed to A: not pass the eave, B: pass the eave, C: re-enter the eave after exiting, D: exit and leaving the filmed area.

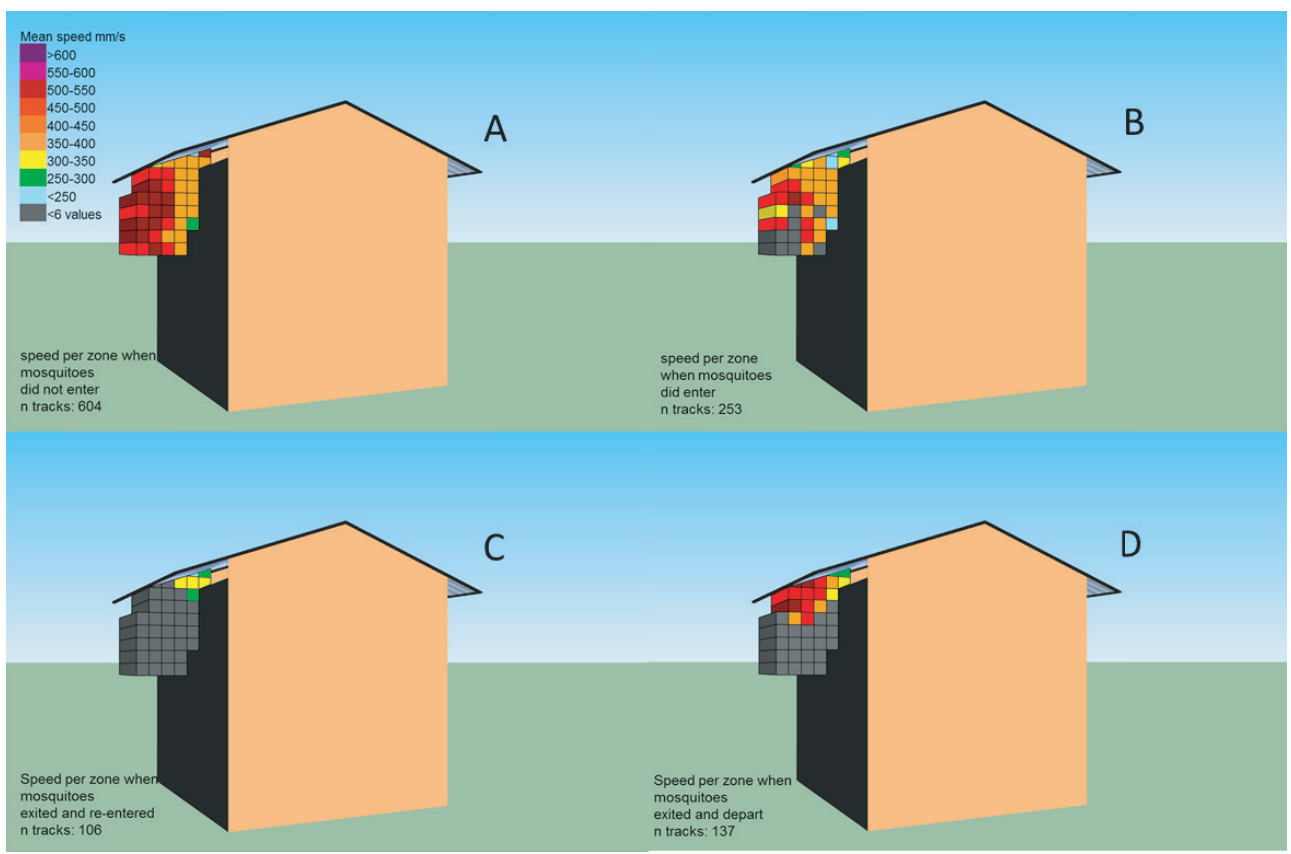

Figure 4.AD Mean flight speeds per zone. The mean flight speed per zone was analysed based on where mosquitoes entered or left the arena with a minimum of six different tracks per zone. 
on where mosquitoes where observed to leave the filmed area (Figure 3.AD). With a random distribution, one may expect that mosquitoes spent $2.6 \%$ in each zone (100/38). Mosquitoes that did not enter the eave showed little variation in time spent between the zones, with a maximum of $6.9 \%$ spent in one of the lower zones close to the wall ( $n$ tracks 604, Figure 3.A). This is in contrast to mosquitoes that were observed to enter the arena: $49.6 \%$ of the time was spent in three zones close to the eave and $82.8 \%$ within the 11 zones closest to the eave ( $n$ tracks 253, Figure 3.B). Mosquitoes that were observed to exit and re-enter the eave did not move far away and spent $95.0 \%$ of the time within four zones close to the eave ( $n$ tracks 106, Figure 3.C). Departing mosquitoes show a gradient in time spent close to the eave, moving away from the eave and following the roof. In total $92.1 \%$ depart and disappear from view via these upper zones ( $n$ tracks 137, Figure 3.D).

Mosquito flight speed and the angular change of heading were analysed separately, depending on where a flying mosquito would leave the filmed area around the house. The mean flight speed over an entire track was lower for mosquitoes that came into view and entered the eave compared to mosquitoes that did not enter, or the ones that exited and departed (379 vs 444 and $412 \mathrm{~mm} / \mathrm{sec}$, respectively; Kruskal Wallis, pair-wise comparisons, $p<0.05$, see Table 1). Lowest flight speeds were

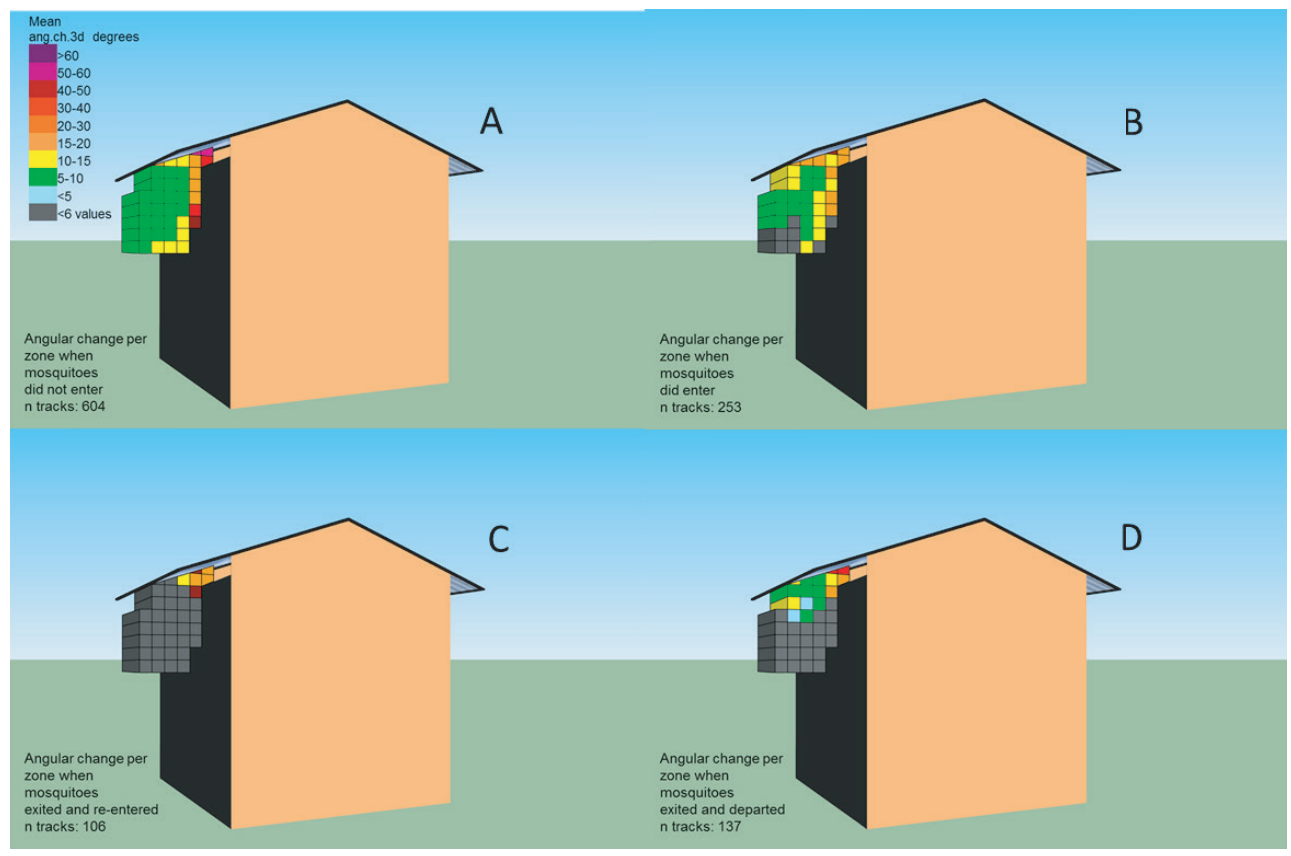

Figure 5.AD Mean angular change in 3D per zone measured in degrees

Higher values indicate a more convoluted flight path. The means per zone were analysed based on where mosquitoes entered or left the arena with a minimum of six different tracks per zone. 


\section{Chapter 5}

recorded for mosquitoes that exited and re-entered the house with on average 291 $\mathrm{mm} / \mathrm{sec}$, (Kruskal Wallis, pair-wise comparisons, $p<0.05$, see Table 1). Figure 4A-D visualizes the mean speeds per zone at different distances from the eave, averaged per mosquito with a minimum of six data points (= the mean is calculated over a minimum of six mosquitoes) per zone and shows a general pattern in which speeds diminished by nearly $50 \%$ when flying closer to the eave. This pattern is visible for both entering and exiting mosquitoes (Figure 4.B and D).

The directness of flight is presented in Figure 5.A-D and shows higher convoluted tracks in zones close to the eave and near the wall or roof of the house. As a result, the mean angular change in 3D over the entire track was highest for mosquitoes that exited and re-entered the house. The mean values did not differ between mosquitoes that came into view and entered compared to mosquitoes that exited and departed (Kruskal Wallis, pair-wise comparisons, $p>0.05$, see Table 1).

\section{Discussion}

This study describes the house entry and exiting behaviour of mosquitoes that were not intercepted by entry/exit traps and not impeded by physical obstacles when passing through the eave. Flight trajectories and their related parameters were different depending on whether mosquitoes were entering, exiting or moving away from a house. However, for all distinct groups, the majority of the mosquitoes that entered or exited the trackable area did this at eave level and spent just a few seconds there. There were no clear indications that mosquitoes arrived at low altitude and moved upwards along the wall towards the eave. This occurred most likely outside the range of the cameras, a few metres away from the house as suggested by Snow (1987). More information was gathered than in studies with interception traps, as with the filming techniques used in this study it was possible to distinguish the flight paths of mosquitoes that entered the house and of those that did not enter and moved away. One can only speculate on the successive behaviour of mosquitoes that did not enter. They could either return into view and eventually enter or move towards other openings that were not filmed and a proportion would not have been in a physiological state of host seeking (Bodin et al., 2009; Foster \& Takken, 2004). For those not host seeking, the endophilic nature of this malaria vector may still have caused movements towards the house and it would require individual tracking of mosquitoes in order to study whether this group would use different cues and/or routes to locate and enter a house (Snow, 1987; Webster et al., 2015).

The standardized synthetic lure used did not include a heat source, however it proved to be able to lure mosquitoes into houses as if they were occupied by human 


\section{Visualization of house-entry behaviour}

beings, as shown in previous studies (Mukabana et al., 2012; Okumu et al., 2010). Heat was considered as a relevant host cue with respect to mosquito landing and of lesser value for house entry, as it has been shown that at a distance $>15 \mathrm{~cm}$ the effect of heat is no longer evident (Spitzen et al., 2013; van Breugel et al., 2015). Although there were no plants growing inside the screen house, the mosquitoes flew in an environment surrounded by plants and occupied buildings were present within $100 \mathrm{~m}$. In other words, there were many competing olfactory cues that had the potential to interfere with a mosquito flight path towards the experimental house.

Mosquitoes that were associated with eave entry spent most of their time within proximity of the eave. Although there was a free passageway to fly through the eave, flight speed decreased nevertheless. This can be explained by short collisions with the walls or ceiling and/or part of the exploration strategy as earlier observed for An. gambiae during their response to host cues (Spitzen et al., 2013) and recently supported in a study on flight behaviour near bed nets (Parker et al., 2015). The same arguments seem valid for the increase in angular change while getting close to the eave. These observations may not seem surprising in themselves, but they raise questions on how this behaviour would change in the presence of disrupting odours (Menger et al., 2015) or if eaves are (partly) blocked (Kirby, 2013; Tusting et al., 2015). Therefore, in field situations, what would be the giving-up time of hostseeking mosquitoes and how long would a mosquito be in contact with treated (netting) material impregnated with fungicides or insecticides (Gatton et al., 2013; Mnyone et al., 2010; Parker et al., 2015; Spitzen et al., 2014)?

Previous studies showed that the presence of (competing) human volunteers inside an experimental house did not affect the results of odour-baited outdoor traps catches (Jawara et al., 2009). During the present study, odours were released inside the house, similar to those emanating from a human, and it would be interesting to find out, using advanced tracking techniques, at what altitude mosquitoes fly further away from the house as a detailed follow-up study by Gillies and Wilkes (1974a) and Snow (1987). As mentioned by several authors, optimal trapping height and thereby other interference tools too, vary between mosquito species and trap design (Gillies \& Wilkes, 1974b; Hiscox et al., 2014; Jawara et al., 2009).

House-exiting mosquitoes seemed to follow the roof edge with increasing speed and a more direct flight while moving away from the house. The authors are not aware of other data on free-flying mosquitoes that have described such behaviour. Mosquitoes that left the house had not obtained a blood meal and were likely to move on in search of other host cues. 


\section{Chapter 5}

House design and its geographical positioning influences air currents and odour plume structures (Cardé \& Willis, 2008; Kirby, 2013). This study presents the results of a house in a semi-field setting, however, recent developments in filming techniques will make it possible to upscale the sample size and to conduct tests in more rural settings. High-speed cameras with increased resolution will allow filming of a larger sample area where fast-moving multiple insects can be tracked (Parker et al., 2015; M Lankheet and JS unpublished data). Ideally, next-generation tracking systems are interlinked with ambient weather data in order to correct for prevailing airflows on the actual airspeed and flight direction of the mosquito. Further developments in tracking techniques could reduce the need for specially constructed experimental houses and enhance knowledge of free-flying mosquitoes (Andriessen et al., 2015; Oxborough et al., 2015) to improve vector intervention tools.

\section{Conclusion}

The malaria mosquito An. gambiae s.s. approaches houses at eave level in a wide, near-horizontal arc. More than half of the examined mosquitoes that approached the house did not proceed to enter the eave. The obtained knowledge on house entry behaviour can be exploited for the development of intervention tools that focus on the disruption of vectors that enter human-occupied buildings. Such interventions should focus on interferences at eave level, with the knowledge that An. gambiae s.s. often passes this route in only a few seconds.

\section{Acknowledgements}

All staff members at the Thomas Odhiambo Campus of the International Centre of Insect Physiology and Ecology in Mbita are gratefully acknowledged for their assistance, including the provision of mosquitoes. We thank Kees Spoor for his support in adjusting the Matlab code to perform batch analysis. Alex Hiscox and David Menger assisted during the experimental phase and are thanked for valuable discussions. This study was supported by the COmON Foundation, The Netherlands. 


\section{Visualization of house-entry behaviour}

Additional file 1. Animation of the experimental set-up including a selection of representative video images and tracking results.

Available via https://malariajournal.biomedcentral.com/articles/10.1186/s12936-016-1293-7.

Additional file 2 Mean time spent per zone

Means calculated over the total time per zone of each individual with a total track length of six frames (0.24 s). $n$ tracks represent the number of times a zone was visited by different mosquitoes. The solid fills highlight the value in the cell. A longer bar represent a higher value.

\begin{tabular}{|c|c|c|}
\hline \multicolumn{3}{|c|}{ Arrive Not Enter } \\
\hline & Mean (s) & n tracks \\
\hline & 0.10 & 6 \\
\hline & 0.17 & 7 \\
\hline & 0.19 & 9 \\
\hline & 0.58 & 34 \\
\hline & 0.57 & 70 \\
\hline & 0.58 & 106 \\
\hline & 0.51 & 91 \\
\hline & 0.32 & 107 \\
\hline & 0.51 & 115 \\
\hline & 0.38 & 66 \\
\hline & 0.28 & 107 \\
\hline & 0.32 & 127 \\
\hline & 0.39 & 94 \\
\hline & 0.29 & 111 \\
\hline & 0.23 & 120 \\
\hline & 0.30 & 154 \\
\hline & 0.27 & 76 \\
\hline & 0.26 & 117 \\
\hline & 0.25 & 111 \\
\hline & 0.34 & 145 \\
\hline & 0.13 & 32 \\
\hline & 0.25 & 99 \\
\hline & 0.22 & 100 \\
\hline & 0.42 & 136 \\
\hline & 0.11 & 46 \\
\hline & 0.23 & 96 \\
\hline & 0.21 & 93 \\
\hline & 0.28 & 94 \\
\hline & 0.15 & 49 \\
\hline & 0.22 & 57 \\
\hline & 0.21 & 104 \\
\hline & 0.13 & 36 \\
\hline & 0.17 & 45 \\
\hline & 0.19 & 46 \\
\hline & 0.28 & 75 \\
\hline & 0.19 & 40 \\
\hline & 0.15 & 30 \\
\hline & 0.13 & 10 \\
\hline
\end{tabular}

\begin{tabular}{|c|c|c|}
\multicolumn{3}{|c|}{ Arrive and Enter } \\
\hline Zone & Mean (s) & n tracks \\
\hline-2 & 0.18 & 58 \\
-1 & 0.12 & 107 \\
0 & 0.21 & 78 \\
1 & 0.49 & 248 \\
2 & 0.48 & 130 \\
3 & 0.44 & 162 \\
4 & 0.58 & 59 \\
5 & 0.28 & 126 \\
6 & 0.37 & 75 \\
7 & 0.52 & 32 \\
8 & 0.31 & 72 \\
9 & 0.23 & 97 \\
10 & 0.31 & 36 \\
11 & 0.25 & 48 \\
12 & 0.21 & 58 \\
13 & 0.23 & 53 \\
14 & 0.17 & 18 \\
15 & 0.22 & 40 \\
16 & 0.20 & 40 \\
17 & 0.22 & 32 \\
18 & 0.09 & 6 \\
19 & 0.13 & 28 \\
20 & 0.20 & 17 \\
21 & 0.39 & 22 \\
22 & 0.06 & 13 \\
23 & 0.23 & 12 \\
24 & 0.14 & 11 \\
25 & 0.45 & 11 \\
26 & 0.11 & 6 \\
27 & 0.22 & 5 \\
28 & 0.15 & 13 \\
29 & 0.09 & 3 \\
30 & 0.12 & 8 \\
\hline & 0.16 & 2 \\
33 & 0.44 & 8 \\
\hline
\end{tabular}

Exit and Re-enter
\begin{tabular}{|c|c|c|}
\hline Zone & Mean (s) & $\mathrm{n}$ tracks \\
\hline-2 & 0.21 & 50 \\
-1 & 0.18 & 84 \\
0 & 0.19 & 49 \\
1 & 0.58 & 71 \\
2 & 0.61 & 8 \\
3 & 0.61 & 11 \\
4 & 1.08 & 3 \\
5 & 0.16 & 2 \\
6 & 0.64 & 2 \\
7 & 0.40 & 3 \\
8 & 0.30 & 2 \\
10 & 0.04 & 1 \\
11 & 0.12 & 2 \\
\hline
\end{tabular}

Exit and Depart

\begin{tabular}{|c|c|c|}
\hline Zone & Mean $(\mathrm{s})$ & $\mathrm{n}$ tracks \\
\hline-2 & 0.17 & 51 \\
-1 & 0.17 & 83 \\
0 & 0.18 & 76 \\
1 & 0.46 & 136 \\
2 & 0.31 & 113 \\
3 & 0.51 & 25 \\
4 & 0.25 & 84 \\
5 & 0.26 & 34 \\
6 & 0.33 & 5 \\
7 & 0.26 & 55 \\
8 & 0.27 & 47 \\
9 & 0.30 & 11 \\
10 & 0.18 & 2 \\
11 & 0.19 & 36 \\
12 & 0.24 & 22 \\
13 & 0.32 & 4 \\
14 & 0.16 & 2 \\
15 & 0.21 & 24 \\
16 & 0.27 & 8 \\
17 & 0.66 & 2 \\
18 & 0.08 & 1 \\
19 & 0.19 & 9 \\
20 & 0.22 & 5 \\
21 & 0.88 & 2 \\
22 & 0.31 & 4 \\
23 & 0.14 & 2 \\
24 & 0.24 & 4 \\
25 & 0.28 & 2 \\
26 & 0.13 & 3 \\
27 & 0.04 & 1 \\
28 & 0.21 & 3 \\
30 & 0.20 & 1 \\
32 & 0.36 & 2 \\
\hline
\end{tabular}




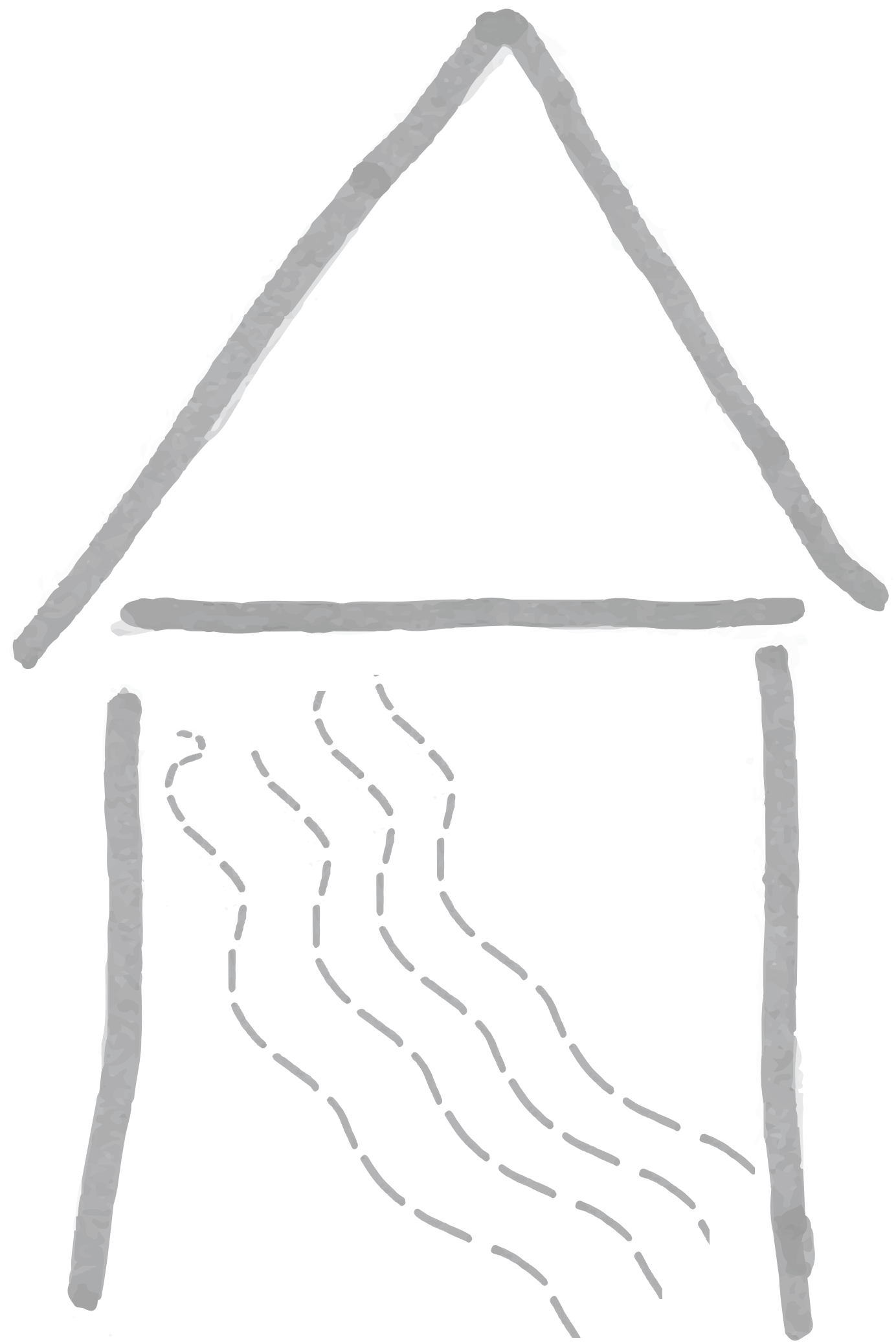




\section{Chapter 6}

\section{Effect of insecticide-treated bed nets on house-entry by malaria mosquitoes: The flight response recorded in a semi-field study in Kenya}

Jeroen Spitzen, Teun Koelewijn,

W. Richard Mukabana, Willem Takken 
Insecticide-treated nets are currently a major tool to reduce malaria transmission. Their level of repellency affects contact of the mosquito with the net, but may also influence the mosquito's entry into the house. The response of host-seeking malaria mosquitoes approaching the eave of an experimental house was recorded within a large screen house. We compared entry- and exit rates in relation to the presence in the house of different insecticide-treated bed nets (ITNs) with an untreated net. Mosquitoes were lured towards the house by dispensing a synthetic host-odour blend from within the net in the house. Complementary WHO bioassays revealed that the treated nets caused high knock-down- and mortality responses to the Anopheles gambiae sensu stricto strain tested. The proportion of mosquitoes that came into view of the cameras and subsequently entered the house did not differ between treated nets and the untreated net. Treated nets did not affect proportions of mosquitoes that exited the house and departed from view around the eave. However, the percentage of house-leaving and re-entering mosquitoes when an insecticide- treated net was present, was lower than in the presence of an untreated net. Our results indicated that there was no spatial repellent effect from pyrethroid-treated nets that influences house-entry at eave level. It is argued that the toxic effect of treated bed nets resulted in a reduced number of mosquitoes re-entering the house, which could thereby affect malaria transmission in neighbouring, unprotected houses. 


\section{Effect of insecticide-treated bed nets on house-entry}

\section{Introduction}

Declines in malaria cases over the last decade are mainly attributed to the use of insecticide- treated nets (ITNs), indoor residual spraying (IRS) and better case management (Bhatt et al., 2015). Increased resistance against insecticides and drugs is considered a major threat to the ability to sustain or further decrease malaria and therefore the continuous assessment of the efficacy of existing tools and the development of new products have been put high on the agenda (World Health Organization, 2016a). For example, lure-and kill technologies, repellents and genetic tools promise to provide tools complementary to the existing insecticidebased strategies (Benelli \& Mehlhorn, 2016; Hammond et al., 2016; Homan et al., 2016; von Seidlein et al., 2017).

ITNs have been proven effective by reducing contact between humans and mosquitoes infected with the Plasmodium parasite (Lengeler, 2004a; Lindsay et al., 1989). The effectiveness of ITNs is dependent on the susceptibility of the local mosquito population to the chemicals used (e.g. Malima et al. (2009)). In addition, house design and the quality of housing are critical (Tusting et al., 2015). The design of houses together with the use of IRS and the presence of ITNs inside houses are other important factors influencing the vector capacity of mosquitoes (Grieco et al., 2000; Koffi et al., 2015; Massue et al., 2016). Continuous monitoring of intervention tools is necessary to measure whether the methods used are still effective or can be optimized. Behavioural adaptions of malaria vectors to control measures have been observed, but there are few studies done that provide supporting data (Gatton et al., 2013; Mathenge et al., 2001; Takken, 2002). Such adaptations could reinforce residual malaria transmission through mosquitoes biting outdoors. This is of concern for further improving malaria control or eradication (Bradley et al., 2016; Killeen, 2014; Killeen et al., 2016; Russell et al., 2013).

Early studies on the effectiveness of ITNs reported the repellent and killing effect of pyrethroids on these nets (Lengeler, 2004a; Miller et al., 1991). Malima et al. (2009) reported that carbosulfan, a carbamate, prevents mosquitoes from entering a house. Tungu et al. (2010) found a deterrent effect of unwashed PermaNet 3.0, but not for PermaNet 2.0 or washed nets. Behavioural data supporting these effects are scarce (Miller \& Gibson, 1994), and conclusions on repellent effects sometimes differ from studies based on indirect behavioural observations that rely on collections from experimental huts (Siegert et al., 2009). More recent studies implemented behavioural recording techniques, and in these studies the repellent effect of pyrethroids is less evident (Cooperband \& Allan, 2009; Parker et al., 2015; Spitzen et al., 2014; Sutcliffe \& Colborn, 2015; Sutcliffe \& Yin, 2014). The change 


\section{Chapter 6}

in interpretations of the repellency of nets may not only be due to more advanced recording techniques. The production process of bed nets has also evolved and repellent effects described earlier could possibly be explained by the emulsifiers used to impregnate the nets (Lindsay et al., 1991). Current nets often lack these components and the active ingredients are incorporated into the net (Spitzen et al., 2014). There is an urgent need for evidence-based studies on the repellent effect of pyrethroids on bed nets, given the rapid development of insecticide resistance in a large number of malaria vectors (Hemingway et al., 2016; Ranson \& Lissenden, 2016; World Health Organization, 2016a).

Recent advances in tracking techniques make it possible to provide detailed information on flight behaviour around hosts, bed nets and human dwellings without the need for interception traps (Angarita-Jaimes et al., 2016; Parker et al., 2015; Spitzen et al., 2016; Spitzen et al., 2014; Sutcliffe \& Colborn, 2015). We investigated if the presence of three different types of pyrethroid-treated nets affected houseentry behaviour of mosquitoes by recording their flight paths near the eave of an experimental house. We hypothesize that, based on short range studies as mentioned above, mosquitoes approaching the eave do not distinguish between the presence inside an experimental house, of an untreated net or a net with insecticides incorporated in the net fibre. The technique used provides information on mosquito behaviour prior to house entry, without the barrier of an interception trap, and can be further developed to support testing and evaluating new generation ITNs, push-pull systems (Menger et al., 2015) and house designs (Tusting et al., 2016).

\section{Materials and Methods}

Experimental set-up

The study was carried out in West Kenya, Mbita Point township, at the Thomas Odhiambo Campus of the International Centre of Insect Physiology and Ecology (icipe-TOC). We video-recorded the approach and house-entry of female mosquitoes on one side of an experimental house that was constructed inside a large screen house. The experimental house had, like many African houses, a wide gap between the top of the wall and the roof. These so-called 'eaves' measured approximately $15 \mathrm{~cm}$ in height and were found at both sides of the house. Two windows and a door remained closed during the experiment. For technical details about the set-up we refer to Spitzen et al., 2016 and Supplementary material, Figure S1). Exit and entry rates were compared in relation to the presence of different bed nets inside the house: 1) an untreated net, 2) PermaNet 2.0, a long lasting insecticidal net (LLIN) with deltamethrin (dose of $1.4-1.8 \mathrm{~g} / \mathrm{kg} \pm 25 \%$ ) incorporated into a coating on the filaments , 3) PermaNet 3.0, a LLIN with deltamethrin in the side panels $(2.1-2.8 \mathrm{~g} /$ 


\section{Effect of insecticide-treated bed nets on house-entry}

$\mathrm{kg} \pm 25 \%)$ and deltamethrin $(4.0 \mathrm{~g} / \mathrm{kg} \pm 25 \%)$ and piperonyl-butoxide (PBO) (25 g/ $\mathrm{kg} \pm 25 \%)$ in the top panel and 4) Olyset, a LLIN with permethrin (20 g/kg $\pm 15 \%)$ incorporated into the filament material. Bed nets were kindly provided by Vestergaard Frandsen SA (Lausanne, Switzerland) or obtained from the local market in Kenya. The samples were tested in random order and kept strictly separate in aluminium bags (Lifesystems $®$, United Kingdom) between experiments. Host odour was standardized using an attractive synthetic blend based on procedures described by Menger et al., (2014b) and Smallegange et al., (2010b) and consisted; Ammonia 2.5\% (v/v), L-(+)-lactic acid 85\% (w/w), Tetradecanoic acid $0.0025 \mathrm{~g} / \mathrm{ll}$, 3-methyl-1butanol $0.000001 \%(\mathrm{v} / \mathrm{v})$, Butan-1-amine $0.001 \%(\mathrm{v} / \mathrm{v})$ including $\mathrm{CO}_{2}$ produced via sugar fermenting yeast which was dispensed from inside the bed net using an MM-X trap (Supplementary Figure S1).

\section{Experimental procedures}

Mosquitoes

Mosquitoes were obtained from the Anopheles gambiae Giles sensu stricto (Mbita strain) insectaries. Three times a week, the colony was blood fed on a human arm for ten minutes. Rearing procedures are described in Spitzen et al., 2016.

\section{WHO bio-efficacy tests}

The bio-efficacy of the tested nets (top panels) was examined using 3 min WHO tube assays (WHO, 2006) and these were done with non-blood-fed, 5-7 day old An. gambiae s.s.. Ten replicates per treatment were done with an average group size of five mosquitoes per tube. After exposure, the groups of mosquitoes were placed in a single $1 \mathrm{~L}$ cup and provided with $6 \%$ glucose solution ad libitum. Their knock-down status was measured 60 min post exposure and mortality was recorded after $24 \mathrm{~h}$.

\section{Behavioural assays}

Five experimental nights per treatment were scheduled. For the analysis, we included experimental nights that were video-recorded for four hours only. Twohundred non-blood-fed mosquitoes, 3-8 days old, were selected 8-10 h before release in the screen house and placed in $1 \mathrm{~L}$ plastic cups, provided with a paper towel soaked in water. The MM-X trap dispensed odour from 20:00 h onwards, just before the moment mosquitoes were released and video recorded. At 24:00 $\mathrm{h}$ the cameras were stopped and the bed net was removed from over the trap, allowing the mosquitoes to enter the trap. Mosquitoes caught in the trap were collected and counted the next morning (Spitzen et al., 2016). During control experiments, there was no bed net present and the trap started running at 20:00 $\mathrm{h}$ so that mosquitoes could be trapped from the moment of mosquito release. This treatment was added 


\section{Chapter 6}

to provide basic information on the number of mosquitoes that enter the house in the absence of a bed net. The odour-baited trap thus served as a proxy for a human being asleep in the house. Due to differences in trapping hours, we did not use data from the house without a bed net to compare the entry/exit rates with the other four treatments.

\section{Data analyses}

For mosquitoes that came into view of the cameras, observations of (re-)entry and exit behaviour were compared between treatments using a Generalized Linear Model (GLM) with binomial distribution and logit link function. The effects of sampling day, mean temperature and humidity over $4 \mathrm{~h}$ of filming were estimated and fitted as parameters when significant $(P<0.05)$. The fitted model per response category is presented in Supplementary material, Table S1. A pairwise comparison test of least significant difference (LSD) was used in case a significant effect of net type on entry and exit behaviour was observed. SPSS Statistics version 22 (IBM corp., USA) was used for the statistical analyses.

\section{Results}

WHO bio-efficacy tests

The effect of the various bed nets on the knock-down and mortality of Anopheles gambiae was recorded after exposure to netting in a WHO tube test. Mosquitoes that were exposed to an untreated bed net were not knocked-down (Figure 1). PermaNet 2.0 and PermaNet 3.0 caused a $98.33 \%$ (SE=1.67\%) and 100\% knockdown response, respectively. Knock-down by Olyset was $94.33 \%$ (SE=4.16\%). The number of mosquitoes that were knocked down after exposure to PermaNet 2.0, PermaNet 3.0 and Olyset was significantly higher than with the untreated net (Fisher exact test, $P<0.001, n=105, n=91, n=101$, respectively). There was a significant difference in mortality between the treated nets (Fisher exact test, $P<0.01$ ). An average of $98 \%(\mathrm{SE}=2 \%$ ) died within $24 \mathrm{~h}$ after exposure to either PermaNet 2.0 or PermaNet 3.0. For Olyset, the average mortality after $24 \mathrm{~h}$ was $81.67 \%$ (SE= $7.78 \%)$. Mortality in the group of mosquitoes that was exposed to the untreated net was $14.5 \%$ ( $\mathrm{SE}=5.29 \%)$. The number of mosquitoes that died following exposure to PermaNet 2.0, PermaNet 3.0 and Olyset was significantly different from the untreated net (Fisher exact test, $P<0.001, n=105, n=91, n=101$, respectively).

The effect of net type on house entry

Mosquitoes were successfully recorded for 19 nights, resulting in 5207 tracks of mosquitoes that came into view of the eave area. As 200 mosquitoes were released per night, it is evident that individual mosquitoes came in view multiple times $\left(19^{\star} 200\right.$ 

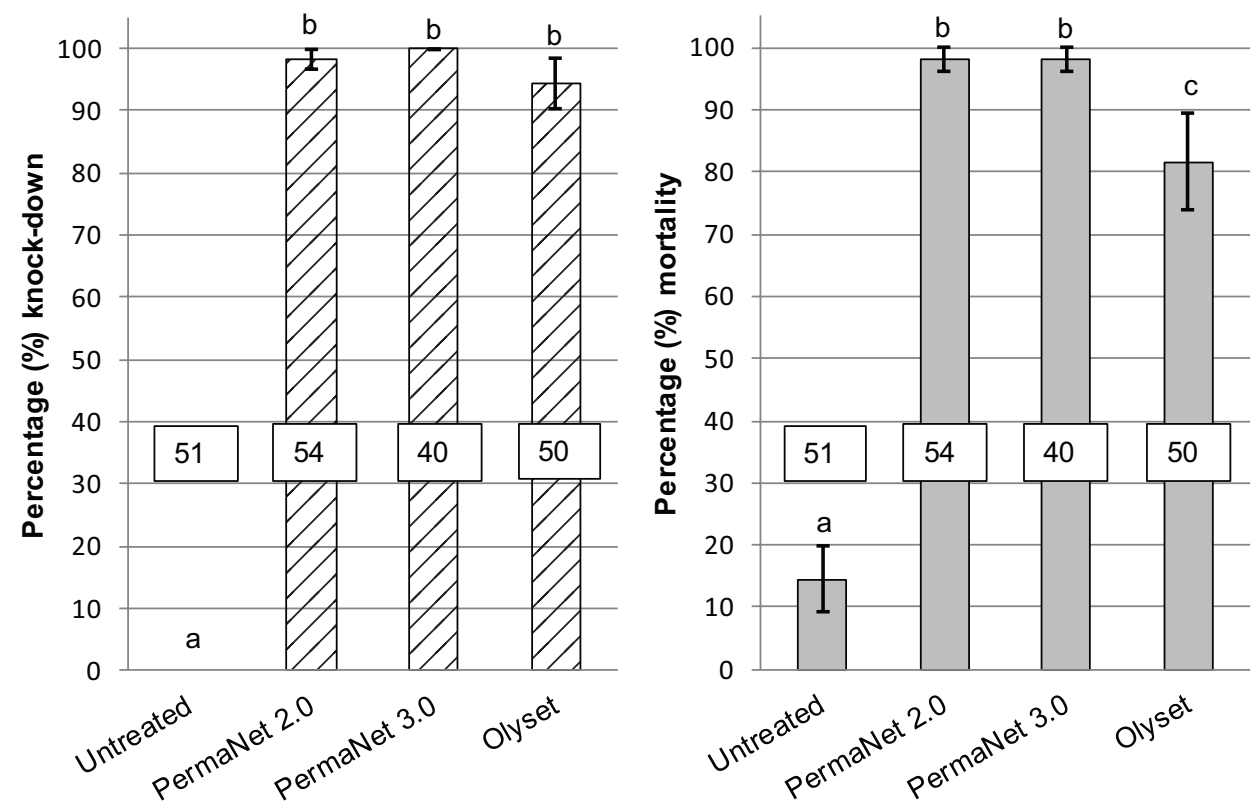

Figure 1. Mean ( \pm SEM) percentage of knock-down (left panel) and mortality rates (right panel) of Anopheles gambiae exposed to an untreated bed net and insecticide-treated bed nets in WHO tube tests for 3 min. Knock-down was measured after one hour and mortality after $24 \mathrm{~h}$. Numbers inside bars represent total number of mosquitoes tested in 10 replicates. Different letters above bars represent significant differences between treatments (Fisher exact test, $\mathrm{P}<0.01$ )

$=3800)$. Not all scheduled replicates could be completed due to irregularities in power supply. Mosquito activity varied between nights ranging from 68 to 635 tracks per night; however the ratio between the four response categories around the eave showed little variation between nights and treatments (see Table 1). The control, with no net, was not included in the GLM as mosquitoes could be removed from the set-up by entering the trap with odour dispenser. We tested if mosquitoes showed different responses to treatments over a six-week time period by comparing responses within the first, second or third block of two weeks. Testing period had no effect and was left out of the model apart from the response category where mosquitoes came in view and entered (GLM, P > 0.05). The mean temperature and humidity over $4 \mathrm{~h}$ per testing day was included in the model where an effect was shown (GLM, P < 0.05). The fitted model for each response category is given in Supplementary Table S1. The number of mosquitoes that flew towards the house and entered, or exited the house and departed from view did not differ between the treatments, including the untreated net (GLM, d.f. $=3$, Wald $X^{2}=4.97 P=0.174$ and Wald $X^{2}=2.14, P=0.544$ respectively). There was a significant effect of net type for mosquitoes that were observed to exit and immediately re-enter the house. More mosquitoes re-entered when an untreated net was present than when there was an insecticide-treated net 


\section{Chapter 6}

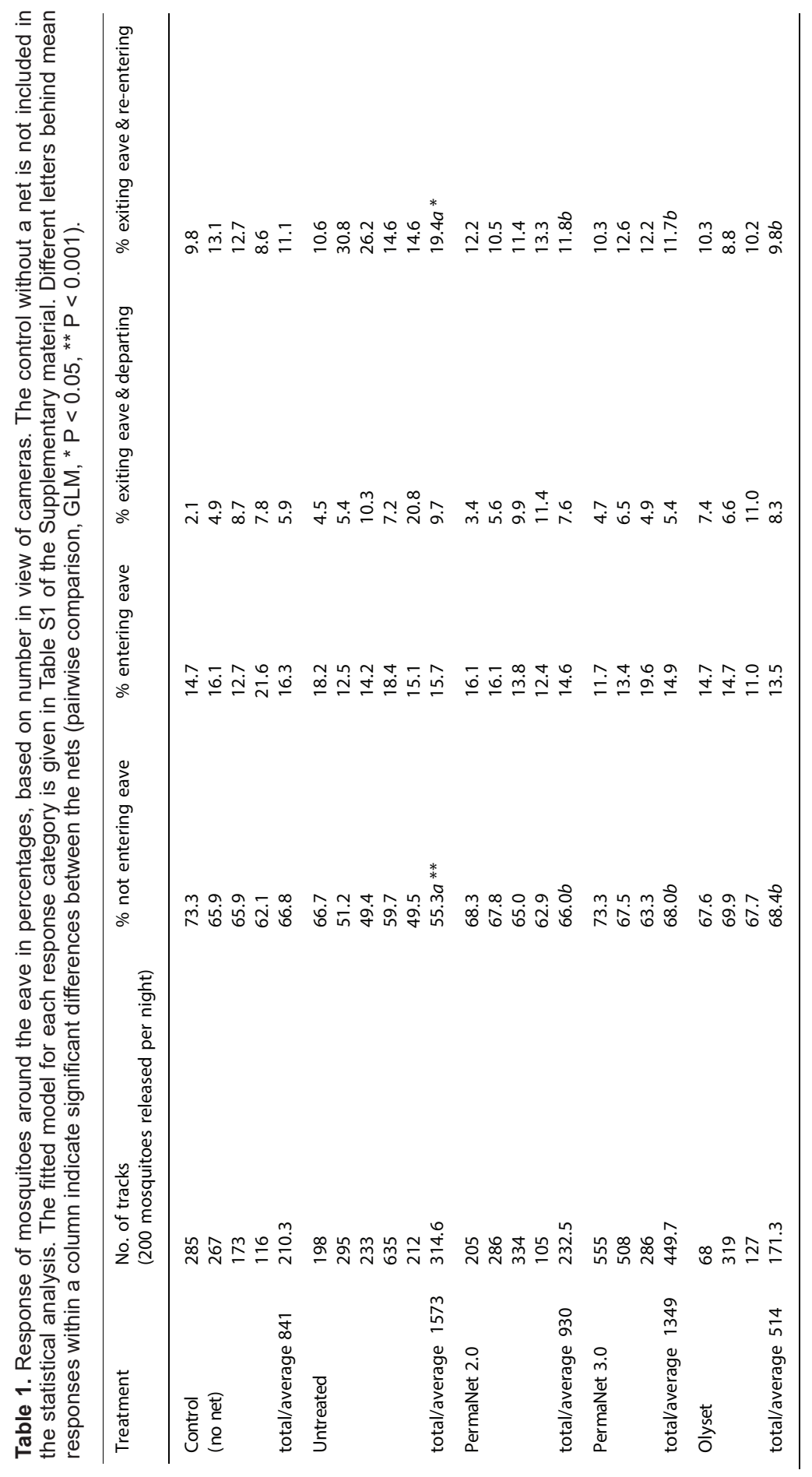




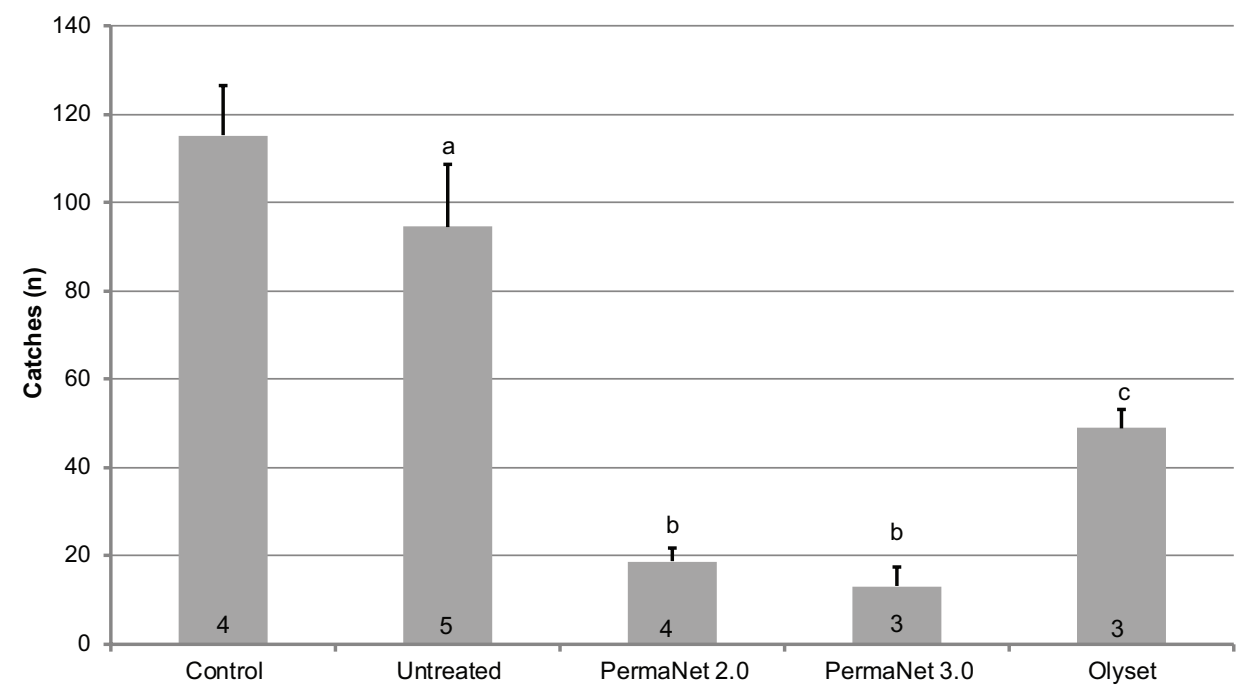

Figure 2. The average number (+SE) of Anopheles gambiae caught in the MM-X traps following exposure to differently treated bed nets. Mosquitoes were trapped from 24:00 h till 10:30 h with the only exception for the control where no net was present. In the control mosquitoes were trapped from 20:00 h till 10:30 h. Different letters above bars indicate significant differences between treatments (GLM, pairwise comparisons, LSD, P <0.05). Numbers inside bars represent number of test nights.

(GLM, d.f. = 1, pairwise comparison, LSD, $\mathrm{P}<0.05$ ). Consequently, the proportion of mosquitoes that came into view but did not enter the house depended on net-type, and pairwise comparisons showed that the proportion that did not enter was lower in the presence of untreated nets than insecticide treated nets, GLM, d.f. = 1, pairwise comparison, LSD, $\mathrm{P}<0.001)$.

Mosquitoes could enter the trap after recording hours (from 24:00 - 10:30 h) when the net was removed from the MM-X trap and hung aside. Catches from the MM-X trap are presented in Figure 2. The day of testing, average temperature and humidity was included in the GLM model but had no effect on numbers caught and were left out of the final model (Supplementary Table S1). Treatment influenced the numbers caught significantly (GLM, d.f. $=3$, Wald $X^{2}=43.01, P<0.001$ ) in that fewer mosquitoes were caught when an insecticide-treated net was present. The presence of an Olyset net had an intermediate effect on catch size. Pairwise comparisons showed that catches with the Olyset net were lower than those from the untreated net (GLM, LSD, P < 0.01), but higher than the PermaNets 2.0 and 3.0 (GLM, LSD, P $<0.05)$. Data from the control without a net was not further analysed because of the difference in sampling time (14.5 h vs. $10.5 \mathrm{~h}$ ). 


\section{Chapter 6}

\section{Discussion}

Mosquitoes showed susceptibility to the tested ITNs with a nearly $100 \%$ knock-down response within one hour after exposure using the WHO tube test. There was no knock-down with the untreated net. Mortality rates after $24 \mathrm{~h}$ were significantly lower for the Olyset net, but greater than $80 \%$ for all treated nets.

The percentage of mosquitoes that exited and re-entered the house was higher when an untreated net was present compared to ITN's. When exposed over a period of $4 \mathrm{~h}$, and given the susceptibility of the mosquitoes to the insecticide-treated nets, it is likely that a proportion of the tested mosquitoes was affected after touching the treated net. This is supported by the number of catches in the MM-X trap after the tested net had been removed and hung beside the odour-baited trap (24:00 - 10:30 h). Fewer mosquitoes were trapped with exposure to ITN treatments than with the untreated net. The deltamethrin containing nets (PermaNet 2.0 and PermaNet 3.0) caused higher knock-down and mortality rates compared to the permethrin-treated net (Olyset) in the tube tests and this is reflected by the higher capture rates following exposure to the Olyset net. As expected, with an untreated net the percentage of mosquitoes that exited and re-entered was higher (Table 1).

The aim of our study was to determine if there was a spatial repellent effect of three different ITNs on house entry of mosquitoes. The number of mosquitoes observed to enter through the eave when an ITN was present inside, was not lower than without a net or when an untreated net was used. Consequently, there was no spatial repellent effect of the tested ITNs at eave level. As recently concluded by Parker et al. (2015), and supported by other studies (Cooperband and Allan, 2009; Spitzen et al., 2014), host seeking is affected by pyrethroid treated nets but not as a result of repellent effects. More likely, mosquitoes are affected by the physical barrier of the net and continue host-seeking as long as they have not had an opportunity to blood feed. A proportion leaves the house to search elsewhere if not affected by the toxic effect of the insecticide after initial contact with the treated nets. A different effect is shown for the carbamate carbosulfan, where Malima et al. (2009) mention a deterrent effect on house entry when bed nets were treated with this class of chemicals.

Earlier studies by Miller et al. (1991) and Darriet et al. (1984) reported a reduction of mosquitoes entering houses when pyrethroid treated bed nets were used. We allocate this difference to the different production process of modern nets, where previous repellent effects were possibly caused by the emulsifiers used to impregnate the nets (Lindsay et al., 1991). LLINs often lack these components and the active ingredients are incorporated into the net which may affect their repellent properties (Spitzen et al., 2014). Besides, possible repellent effects may be overcome by the natural presence of host odours, as indicated by Kongmee et al. (2012) and 


\section{Effect of insecticide-treated bed nets on house-entry}

further discussed by Sutcliffe and Yin (2014). An exception to this theory seems an experimental hut trial using interception traps where the authors report a deterrent effect of unwashed PermaNet 3.0, but not for PermaNet 2.0 or washed nets (Tungu et al., 2010). Interpreting results from studies on the repellent effect of IRS on house entry should also consider the emulsifiers or solvents used, the presence and quality of host odours, and whether the observed effect is a result of toxicity rather than repellency (Grieco et al., 2000; Killeen \& Moore, 2012; Sutcliffe \& Yin, 2014).

Although we could not follow behaviours of individual mosquitoes throughout and focused on the area where most house entry occurs, we obtained some valuable information on the proportion of mosquitoes that moved in and/or out via the eave. Exit and re-entry behaviour could be a result of mosquitoes repeatedly attempting to get to a blood meal while being obstructed from doing so by the bed net. Whether a proportion of these exiting mosquitoes would still suffer from a sub-lethal effect as a result of the insecticides could not be observed in the present study.

When there is no repellent effect of the ITNs, selection for resistance to pyrethroids is even faster than when there is a repellent effect. Mosquitoes that are repelled by an ITN may still be able to find another blood host and survive long enough to contribute to the gene pool of the population (Takken, 2002).

\section{Conclusion}

This study provides further evidence, based on behavioural recordings of mosquitoes, that bed nets with the insecticides incorporated into the netting do not repel mosquitoes. However, ITNs will remain an important barrier to spread the malaria parasite to humans, especially as long as the mosquito population remains susceptible to the insecticides used. Analysing behaviour of vectors in (semi-)field settings can contribute to the development and effectiveness of new intervention strategies, such as push-pull studies (Homan et al., 2016; Menger et al., 2015; Okumu et al., 2012). Increasing the field of view of the cameras and linking outdoor flight behaviours with behaviours inside a house would further benefit the evaluation of repellents or physical barriers.

\section{Acknowledgements}

We thank all staff members at the Thomas Odhiambo Campus of the International Centre of Insect Physiology and Ecology in Mbita for their assistance, including the provision of mosquitoes. Alex Hiscox and David Menger assisted during the experimental phase and are thanked for valuable discussions. We are grateful to Kees Spoor, Enric Frago, Gerrit Gort and Niels Verhulst for their support and advice on the data analysis. This study was supported by the COmON foundation, The Netherlands. We appreciate the suggestions made by anonymous reviewers to improve the original manuscript. 


\section{Chapter 6}

\section{Supplementary material}

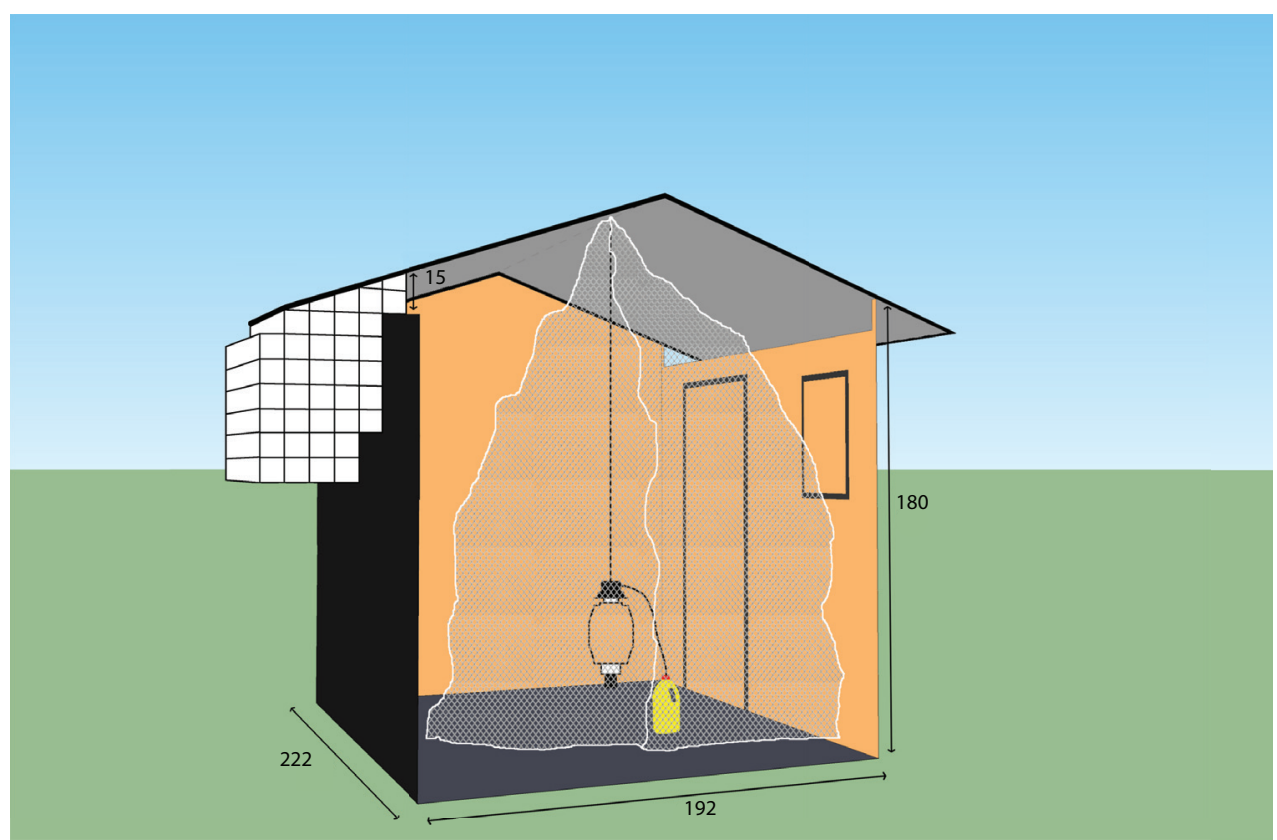

Figure S1

Diagram of the experimental house, with the odour-baited MM-X trap covered by an (insecticide-treated) bed net (source: Spitzen et al., 2016). Measurements in $\mathrm{cm}$. White blocks, with a width of $70 \mathrm{~cm}$, indicate the area of interest filmed by two cameras. 
Effect of insecticide-treated bed nets on house-entry

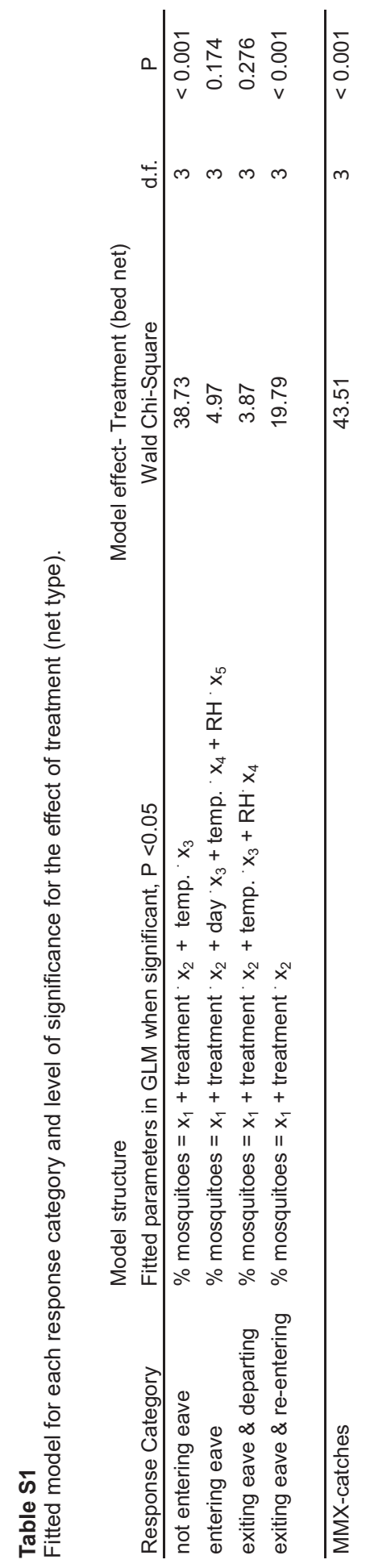





\section{Chapter 7}

\section{Absence of close-range excitorepellent effects in malaria mosquitoes exposed to deltamethrin-treated bed nets}

Jeroen Spitzen,

Camille Ponzio,

Constantianus J. M. Koenraadt,

Helen V. Pates Jamet,

Willem Takken 
Flight behaviour of insecticide-resistant and susceptible malaria mosquitoes approaching deltamethrin-treated nets was examined using a wind tunnel. Behaviour was linked to resulting health status (dead or alive) using comparisons between outcomes from free-flight assays and standard World Health Organization (WHO) bioassays. There was no difference in response time, latency time to reach the net, or spatial distribution in the wind tunnel between treatments. Unaffected resistant mosquitoes spent less time close to $(<30 \mathrm{~cm})$ treated nets. Nettings that caused high knock-down or mortality in standard WHO assays evoked significantly less mortality in the wind tunnel; there was no excitorepellent effect in mosquitoes making contact with the nettings in free flight. This study shows a new approach to understanding mosquito behaviour near insecticidal nets. The methodology links free-flight behaviour to mosquito health status on exposure to nets. The results suggest that behavioural assays can provide important insights for evaluation of insecticidal effects on disease vectors. 


\section{Introduction}

Insecticide-treated bed nets (ITNs) have been the recommended strategy for the prevention of malaria since 2005, and widespread use of ITNs, coupled with accurate case management, has led to a significant reduction in malaria morbidity and mortality (Lim et al., 2011; Steketee \& Campbell, 2010). Synthetic pyrethroids are the only insecticide class currently approved for use on bed nets, mainly because of their relatively low mammalian toxicity, low costs, high residual efficacy, applicability to long-lasting technology, and fast killing effect on mosquitoes. Numerous studies have shown their strong protective effect against bites of infectious malaria mosquitoes (Binka et al., 1996; Lengeler, 2004a, 2004b; Phillips-Howard et al., 2003). Pyrethroids, especially permethrin and deltamethrin (two of the most widely used insecticides on bed nets), are also reported to have a repellent effect (Curtis, 1991; Diabate et al., 2002; Kongmee et al., 2012; Mosha et al., 2008; Singh et al., 1989). However, these (excito-) repellent properties of ITNs are often assumed based on experimental hut studies, and quantified data on mosquito flight behaviour are lacking (Darriet et al., 1984; Killeen \& Smith, 2007; Koudou et al., 2011; Lindsay et al., 1991; Mathenge et al., 2001; Miller et al., 1991).

There can be different interpretations of the term excitorepellency or contact irritancy (Achee et al., 2009; Cooperband \& Allan, 2009; Roberts et al., 2000; White, 2006); for this paper, we follow the description of an excitorepellent by White (2006), which is in line with that of a contact irritant: the power of a chemical, followed by brief tarsal exposure, especially though by brief tarsal exposure, to irritate the insect sufficiently that it moves away before knock-down, even from sub-lethal exposure. Some studies report mosquito avoidance behaviour to pyrethroid-treated bed nets but with no clear observation of a repellent effect (Bogh et al., 1998; Itoh et al., 1986). However, others report repellent effects of deltamethrin following the application as an indoor residual spray (IRS) (Grieco et al., 2000). Experimental hut studies suggest a repellent effect of pyrethroid-treated bed nets towards Anopheles gambiae based on reduced hut entries or forced exit. Current designs of experimental huts do not provide the possibility to study close-range behaviour in detail (Lindsay et al., 1991; Okumu et al., 2012).

World Health Organization (WHO) tunnel tests measure whether mosquitoes will pass a treated net, but do not measure flight behaviour during their approach towards the net (World Health Organization, 2005). WHO guidelines propose to use a guinea pig as a bait for An. gambiae mosquitoes, but for studying host-seeking responses in relation to treated nets, it seems more applicable to use human bait for studying the anthropophilic mosquito An. gambiae (Mboera \& Takken, 1999). 


\section{Chapter 7}

The present study was undertaken to assess the close-range behaviour of the malaria mosquito An. gambiae when exposed to insecticide-treated netting in a wind tunnel. The host-seeking behaviour of insecticide-susceptible and resistant mosquitoes was recorded by video cameras while the mosquitoes flew upwind to human odorants shielded from the mosquitoes by insecticide-treated netting. Recorded flight behaviours were linked to individual mosquito health status (i.e., dead or alive) after exposure to the nets. The bioefficacy of the nets was validated by comparing the wind tunnel data of free-flying mosquitoes with that of standard WHO bioassays for insecticide-treated materials.

\section{Material and Methods}

Mosquitoes

The Wageningen laboratory strain of An. gambiae Giles sensu stricto var. Suakoko was used as well as a resistant strain of An. gambiae (VKPR) that originated from Burkina Faso. The Suakoko mosquito strain is $100 \%$ susceptible to pyrethroids, and the VKPR strain (insecticide susceptibility profile is available from the authors) is pyrethroid-resistant and homozygous for the $k d r$ mutation L1014F. Both strains were kept in $30 \times 30 \times 30 \mathrm{~cm}$ cages at $27 \pm 1^{\circ} \mathrm{C}$, at a relative humidity of $70 \pm 5 \%$ with a 12:12-hour light regime. Adults were provided with a $6 \%$ glucose solution ad libitum, and females were bloodfed using a membrane feeding system by Hemotek (Discovery Workshop, Accrington, United Kingdom). Human blood was obtained from the Sanquin blood bank, Nijmegen, The Netherlands, where it had been donated by volunteers. Larvae were reared in 1.5-L trays filled with tap water and fed Tetramin (Tetrawerke, Melle, Germany) fish food.

\section{Wind tunnel and automated tracking}

Conditioned air $\left(27^{\circ} \mathrm{C} \pm 1^{\circ} \mathrm{C}\right.$ and relative humidity of $70 \% \pm 5 \%$ ) was blown into a wind tunnel $(160 \times 60 \times 60 \mathrm{~cm})$ with a flow rate of $10 \mathrm{~cm} / \mathrm{second}$. The side walls and floor of the arena were constructed of black recycled polycarbonate, and the ceiling was made of transparent Lexan polycarbonate (WSV Kunststoffen, Utrecht, The Netherlands). The wind tunnel was illuminated from the downwind side using Four Tracksys (Nottingham, United Kingdom) infrared light units. Each unit contained an array of 90 infrared light-emitting diodes (LEDs) emitting light with peak output at $880 \mathrm{~nm}$. To optimize the contrast of the illuminated mosquito wings against the background, another four infrared lights containing 168 LEDs (>920 nm) each (Reinaert Electronics, Amsterdam, The Netherlands) were added in the same line as the Tracksys infrared lights. The experimental room was illuminated using a 15Watt light bulb facing away from the test arena to provide visual feedback during flight. Mosquitoes approaching within $60 \mathrm{~cm}$ of the netting were recorded using a 


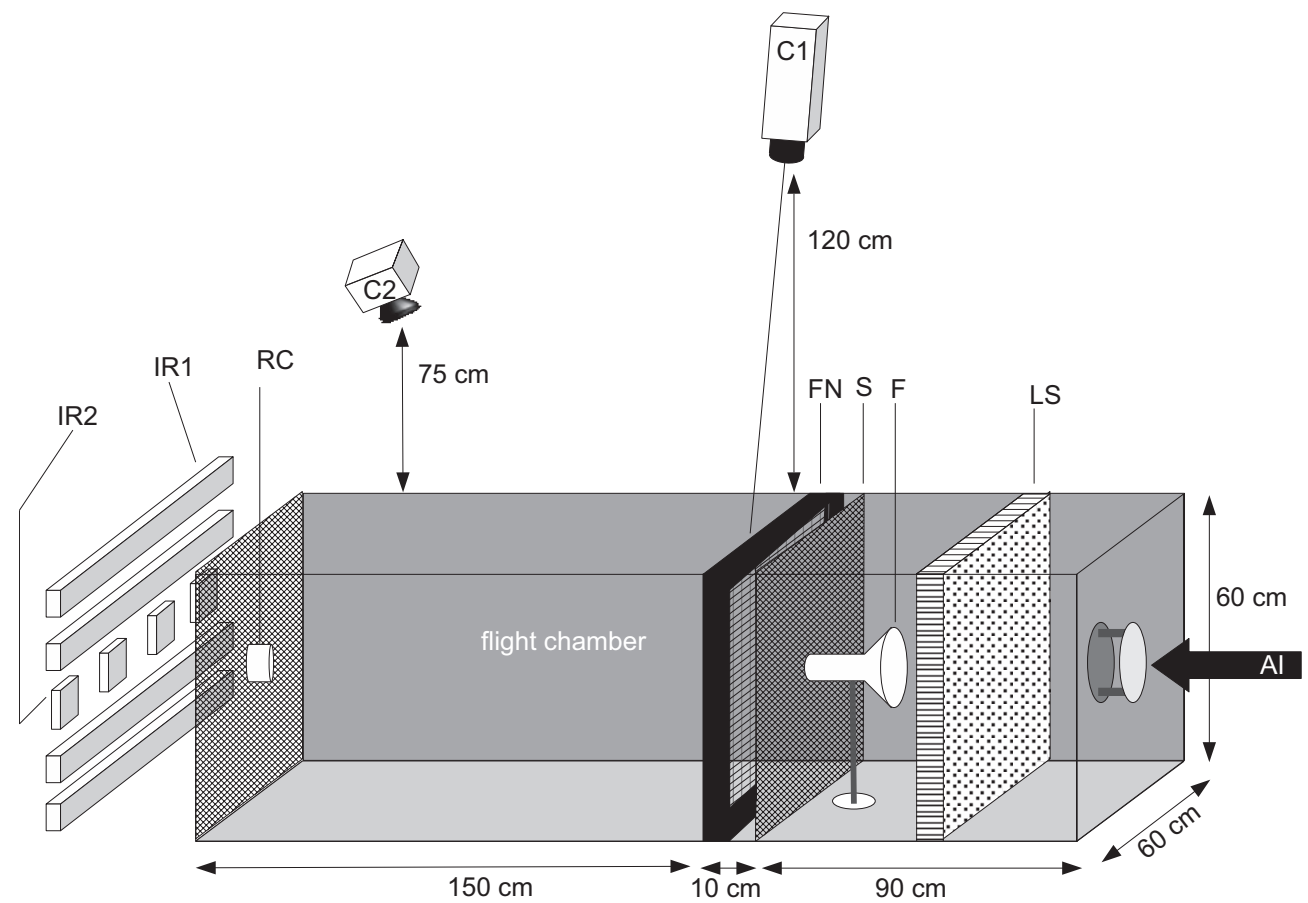

Figure 1. Schematic diagram of the wind tunnel. Air inlet (AI), lamination screen (LS), glass funnel containing heat element $(F)$, mesh screen $(S)$, framed net $(F N)$, release cup $(R C)$, cameras (C1 and 2), and infrared lights types 1 (IR1), and 2 (IR2) are shown.

Cohu 4722-2000/0000 monochrome CCD camera (Cohu, San Diego, CA, USA) equipped with a Fuji Non-TV f1.4 9-mm lens. An Ikegami camera (IR49E B/W) with a wide-angle lens (LM3NCM Mp KOWA 3,5/f2.4 C-Mount) was used to obtain a view over the entire flight arena (Figure 1). The position of a mosquito in the arena was tracked offline using Ethovision XT 7 (Noldus Information Technology, Wageningen, The Netherlands).

\section{Tested nets}

Coded netting samples were received from Vestergaard Frandsen SA (Lausanne, Switzerland) and tested in a double-blind fashion. For tracking optimization, black nets were used. The samples were kept strictly separate in aluminium bags (Lifesystems, United Kingdom). The netting codes were revealed at the end of the experimental phase. The nets consisted of black untreated polyester, black polyester coated with deltamethrin $(1.8 \mathrm{~g} / \mathrm{kg}$; deltamethrin netting), and black polyethylene incorporated with deltamethrin at $4 \mathrm{~g} / \mathrm{kg}$ and piperonyl butoxide (PBO) at $25 \mathrm{~g} / \mathrm{kg}$ (deltamethrin+ PBO netting). Netting samples were mounted on a square frame of black polycarbonate that fit tightly in the $60 \times 60-\mathrm{cm}$ wind tunnel; in this way, 50 $x 50 \mathrm{~cm}$ of fabric could be exposed to the mosquitoes. A strip of door brush fixed 


\section{Chapter 7}

the netting to the frame and prevented scratches to the walls. To ensure that the wind tunnel could not be accidentally contaminated with residues of insecticides, the netting was placed $10 \mathrm{~cm}$ from the upwind end of the tunnel and did not touch the walls of the arena. The odour source to which mosquitoes were exposed consisted of a nylon stocking (worn for 24 hours), which was supplemented by carbon dioxide released at $250 \mathrm{~mL} /$ minute (Smallegange et al., 2010b; Spitzen et al., 2008b). The nylon stocking was placed over a $34^{\circ} \mathrm{C}$ heat source to enhance odorant release and simulate the odour of human skin. The odour source and heating system were placed behind the centre of the upwind screen out of reach of the mosquito.

\section{Experimental procedures}

Individual female mosquitoes, aged 5-7 days, were placed in a plastic release container (diameter $=5 \mathrm{~cm}$, height $=3 \mathrm{~cm}$ ) with a piece of wet cotton 13 to 16 hours before the start of the experiment. Wind tunnel tests were performed during the last 4 hours of the scotophase. The nettings were tested using a Latin square design and switched after four to six replicates per testing day. Surgical gloves were worn and replaced with each treatment. In between experiments, the framed nets were stored in the aluminium bags. The release container was placed at the downwind end of the arena and remained closed for 1 minute. The mosquito release system was operated from an adjacent room to avoid the effects of other human odours during the experiment. After release, 3 minutes were allowed for upwind flight response. The response time was recorded and defined as the time in seconds between release and the first appearance within the arena. If mosquitoes entered the flight arena, their flights were recorded for 5 minutes. Directly thereafter, mosquitoes were removed from the wind tunnel using a manual aspirator and transferred to labelled $50-\mathrm{mL}$ cylindrical tubes covered with netting and damp cotton wool with a $6 \%$ sugar solution. Knock-down status was measured after 60 minutes, and survival status was measured at 22-24 hours after the experiment. The wind tunnel was cleaned weekly using cotton wool and a $10 \%$ alcohol solution.

\section{WHO cone and tube tests}

Standard 3-minute WHO cone and tube assays were performed with unfed An. gambiae s.s. (both susceptible and resistant strains) in the same age-group used for the wind tunnel test, (5-7 days old) (World Health Organization, 2005, 2006). Two groups of five female mosquitoes were tested in each tube or cone, with a minimum of seven replicates per treatment. After exposure to the netting inside the cone or tube, both groups of mosquitoes were placed in a single 1-L cup and provided with $6 \%$ glucose solution ad libitum. Their knock-down status was measured 60 minutes post-exposure, and mortality was recorded at 24 hours. These data were used for comparison with survival and mortality in the wind tunnel bioassay. 
Table 1. Zone definitions used for spatial analyses at a range of 0-60 $\mathrm{cm}$ from the netting samples.

\begin{tabular}{ll}
\hline \multicolumn{1}{c}{ Zone } & \multicolumn{1}{c}{ Zone description } \\
\hline $60-150 \mathrm{~cm}$ & Mosquito was more than $60 \mathrm{~cm}$ from the net \\
$30-60 \mathrm{~cm}$ & Mosquito was in a zone from 30 to $60 \mathrm{~cm}$ from the net \\
$0-30 \mathrm{~cm}$ & Mosquito was within $30 \mathrm{~cm}$ of the net \\
Net & Mosquito touched net at upwind side of the wind tunnel \\
On source & Mosquito reached $(5 \mathrm{~cm} \varnothing)$ host odour source at the net \\
\hline
\end{tabular}

Zone widths were measured from the center of the arena at a height of $30 \mathrm{~cm}$ using images from camera 1. To optimize tracking, the angle of this camera was not $100 \%$ in (vertical) line with the arena, and therefore, the zones slightly overlap.

\section{Data analyses}

Data were analysed using Ethovision XT 7 (Noldus Information Technology, Wageningen, The Netherlands; http://www.noldus.com). Five different zones were allocated to the flight arena (Table 1), and a Kruskal-Wallis test was used to examine differences in the mean time spent per zone between treatments. Post-hoc analyses (multiple comparison, Scheffe) were done to reveal significant differences between nets. A threshold for mobile or immobile mosquitoes was set at $>19$ and $<17 \mathrm{~mm} /$ second, respectively. Parameters studied were (1) response (the percentage of responding mosquitoes and the time until response as well as the latency time until first contact with the net) and (2) proportion of time (immobile) on the netting or in different zones of the flight arena. Parameters were analysed in relation to net type, mosquito strain, and mosquito health status. The health status of mosquitoes tested in the wind tunnel assay was compared with the outcomes from the WHO cone and tube assays and registered as unaffected, knocked down, or dead.

Binary logistics (generalized linear model [GLM]) were used to analyse differences in responses between net types and mosquito strain; time-related variables such as response time and latency to touch the net, were analysed using Cox's regression.

\section{Results}

\section{WHO bioassays}

There was no knock-down or mortality in any of the controls. In the tube bioassay with susceptible mosquitoes, 100\% knock-down and 100\% mortality with deltamethrin netting and deltamethrin + PBO netting were observed. In the cone bioassay, $82 \%$ knock-down and $80 \%$ mortality were observed with deltamethrin netting, and $100 \%$ knock-down and 100\% mortality with deltamethrin + PBO netting were observed (Figure 2). With the resistant mosquitoes in the tube assay, $70 \%$ knock-down and $53 \%$ mortality were observed for deltamethrin nets, and $80 \%$ knock-down and $71 \%$ mortality were observed for deltamethrin + PBO netting. In the cone bioassay, $44 \%$ knock-down and $40 \%$ mortality were recorded with deltamethrin netting, whereas $87 \%$ knock-down and $81 \%$ mortality were recorded with deltamethrin + PBO netting. 


\section{Chapter 7}

Susceptible strain
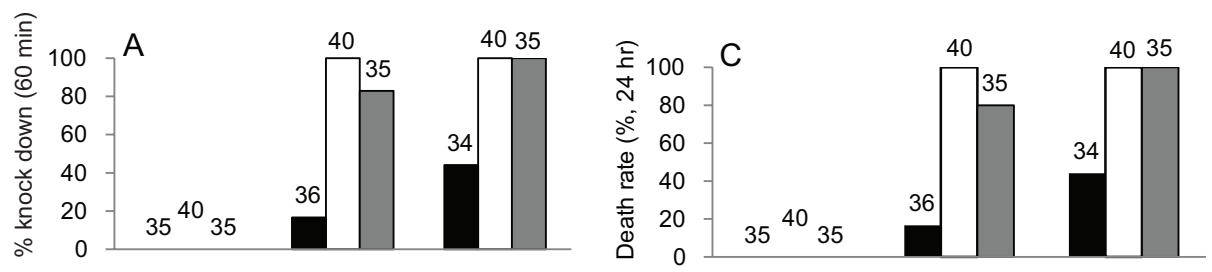

VKPR
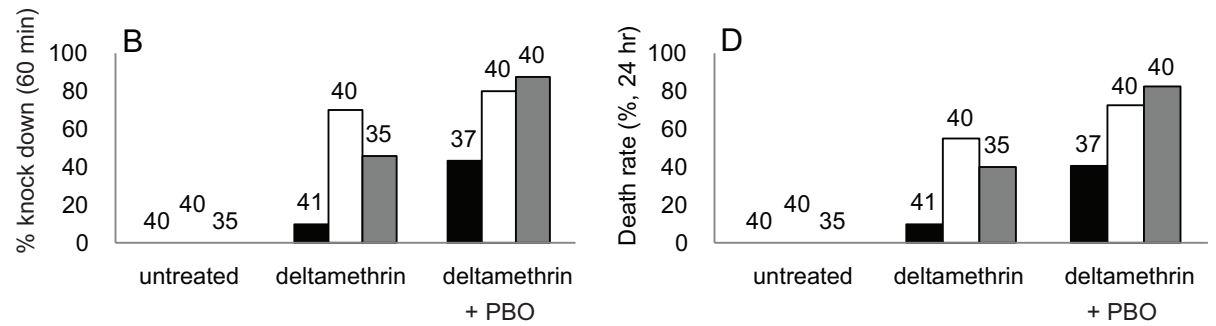

wind tunnel

tube bioassay cone bioassay

Figure 2. (A and B) Percentage of mosquitoes that was recorded knocked down after 1 hour after testing in the wind tunnel or after 3 minutes of exposure in a cylinder tube or WHO cone. $\mathbf{C}$ and $\mathbf{D}$ show the death rate in percentage after 24 hours. Numbers above bars represent number of individuals recaptured in the wind tunnel and total number tested (in groups of five) for the other bio-assays.

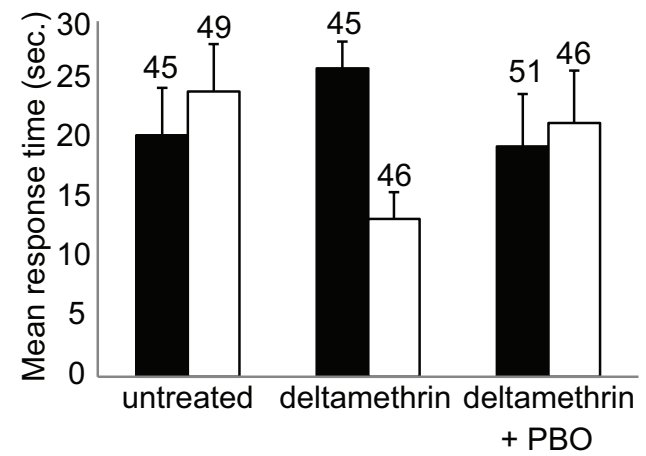

- Susceptible a VKPR

Figure 3. Mean ( \pm SEM) response time in seconds for each treatment in which mosquitoes took off for upwind flight within 3 minutes after being released at the downwind side of the wind tunnel.

\section{Behavioural assays}

Mosquitoes took off for upwind flight in $92.5 \%$ (86.5- $98.1 \%)$ of the cases, and there was no effect of mosquito strain and net type on the number of mosquitoes responding (leaving the release cage; GLM: $P=0.407$ and $P=0.315$, respectively) (Table 2). The mean response time varied between 13.3 and 26.0 seconds (Figure 3). There were no significant differences in response time between the netting samples and mosquito strains (Cox's regression: Wald $=1.40$, degrees of freedom [df] $=2, P=0.497$ and Wald $=0.18$, df $=1, P=0.672$, respectively). 
For mosquitoes that took off for upwind flight, the amount of time spent in the different upwind zones of the wind tunnel (on netting; within $0-30 \mathrm{~cm}$ of the section, within 30 $60 \mathrm{~cm}$ of the section, or farther away than $60 \mathrm{~cm}$ ) was not different between the three netting types for each mosquito strain (Table 3) (Kruskal Wallis, $P>0.05$ ). The spatial distribution of mosquitoes across the wind tunnel was also examined separately for those mosquitoes that were not affected by the insecticides and those mosquitoes that were knocked down (Table 3). The mosquitoes affected by the treatment spent much longer sitting on the net compared with those mosquitoes that were unaffected (Figure 4) and less time in the sections farther than $30 \mathrm{~cm}$ from it (data per section in Table 3). VKPR mosquitoes that were unaffected by the deltamethrin + PBO netting spent significantly less time close to the net and relatively more time at the downwind side of the wind tunnel (Table 3).

Figure 5 shows examples of tracking results for each of the three netting types (control, deltamethrin, and deltamethrin + PBO). Fragments of animated flight tracks are shown in Supplemental Videos 1-3. These tracks were selected on the condition that the mosquito reached the upwind end of the arena, where the odour and heat source was placed behind the netting sample. The percentage of mosquitoes that made contact with the upwind net varied from $41.3 \%$ to $68.6 \%$. The percentage of mosquitoes that reached the net at the site where host cues were released $(5 \mathrm{~cm}$ diameter) ranged from $15.2 \%$ to $32.6 \%$ (Table 2 ). There was no interaction between netting type and mosquito strain in terms of reaching the odour source (GLM: $P=$ 0.241 ) and no difference in response (exiting release container within 3 minutes) between the treatments and the mosquito strains (GLM: $P=0.108$ and $P=0.863$, respectively). Fifty-one percent of mosquitoes of either strain made contact with the upwind untreated net within 5 minutes (Table 2). There was no effect of treatment on the percentage of mosquitoes touching the net, and more susceptible than resistant mosquitoes touched the deltamethrin netting (GLM: Wald $=4.924, P=0.026$ ). Susceptible mosquitoes arrived sooner at the upwind end of the wind tunnel than the resistant strain (Table 2) (Cox's regression: Wald $=5.95$, $d f=1, P=0.015$ ). However, there was no significant difference between the netting types in latency to touch the net (Cox's regression: Wald $=0.71, \mathrm{df}=2, P=0.701$ ).

Of responding mosquitoes, 66.7-89.1\% were recaptured and examined for 60 -minute knock-down and 24-hour survival. None of the mosquitoes exposed to an untreated net in the wind tunnel were knocked down or died within 24 hours after exposure. Of the susceptible mosquitoes exposed to deltamethrin netting, $16.7 \%$ were knocked down and died within 24 hours, whereas $44.1 \%$ of those mosquitoes exposed to deltamethrin + PBO netting were knocked down and died. Of the resistant 


\section{Chapter 7}

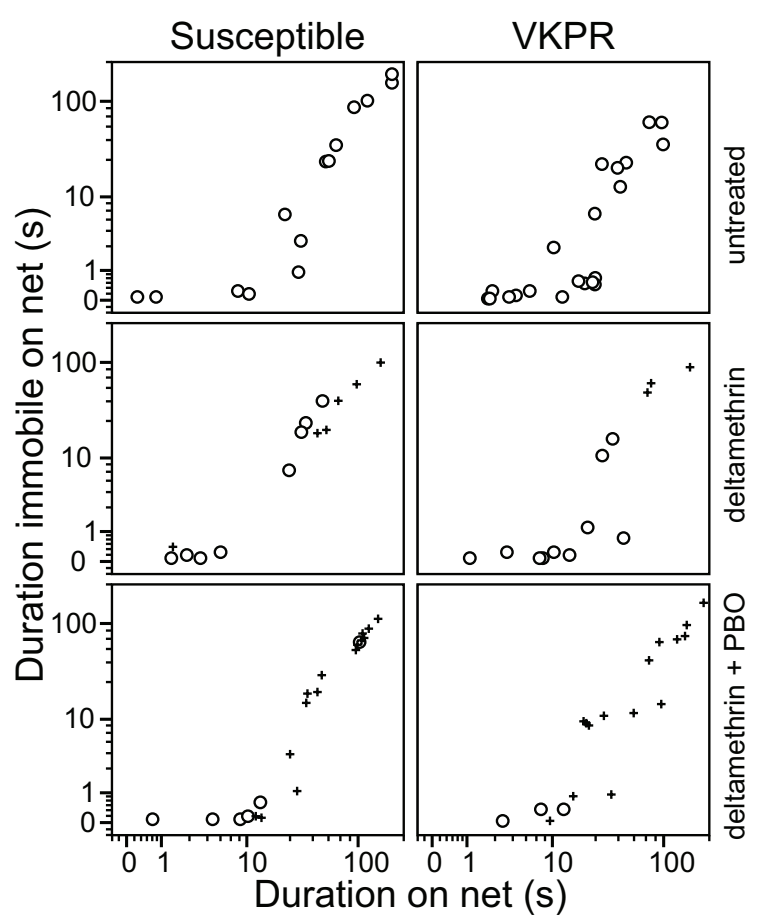

$1 \mathrm{hr}$ health status: o healthy, + knocked down

Figure 4. One-hour health status of both mosquito strains in relation to the duration spent on the net ( $x$ axis) immobile on the net ( $y$ axis) in seconds.

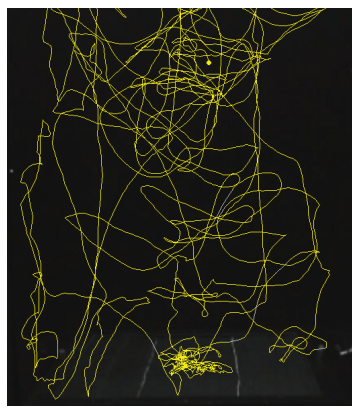

untreated

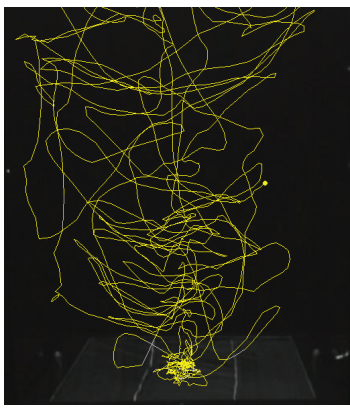

deltamethrin

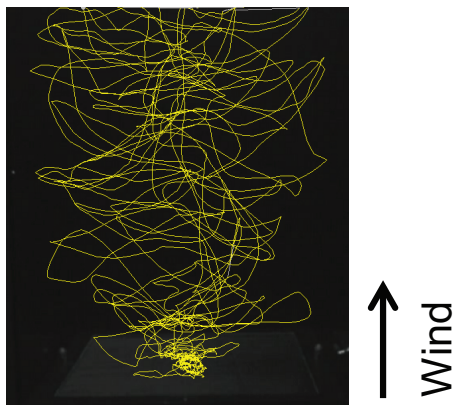

deltamethrin $+\mathrm{PBO}$

Figure 5. Example of tracking results for individual mosquitoes of the susceptible strain approaching three different netting samples. The odour source is located on the upwind side, just behind the netting. The mosquito that contacted the untreated netting was unaffected. The other two showed a 1-hour knockdown response and died within 24 hours after recapture. 


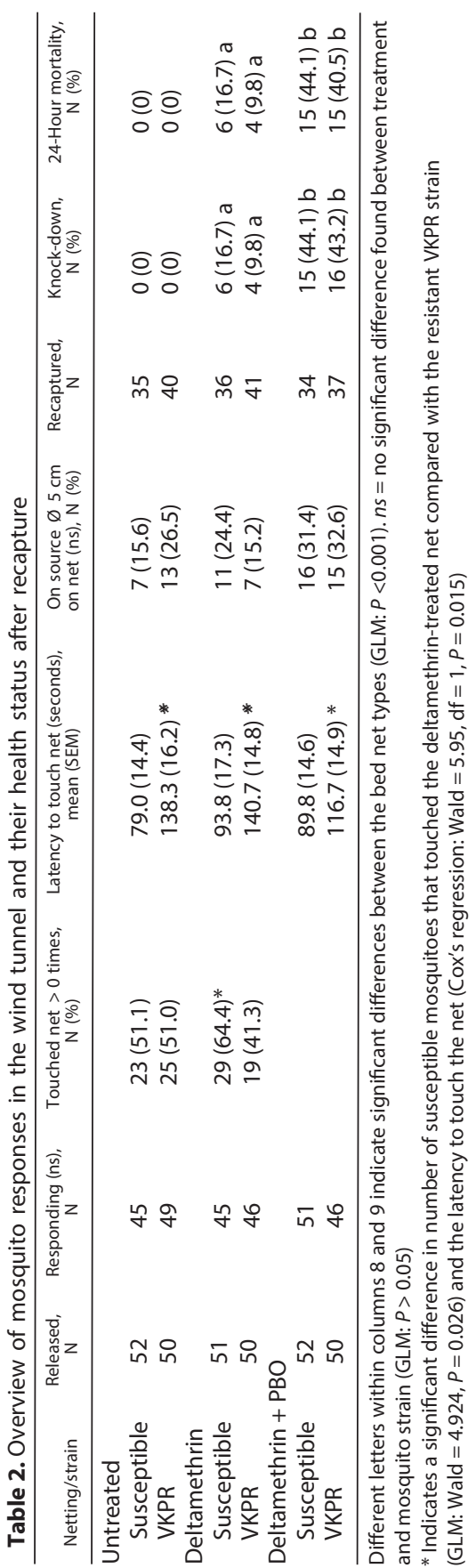

mosquitoes exposed to deltamethrin netting, $9.8 \%$ of mosquitoes were knocked down and died, whereas $43.2 \%$ of mosquitoes were knocked down and $40.5 \%$ of mosquitoes died after exposure to the deltamethrin + PBO netting (Figure 2 and Table 2). There was a clear effect of the time actually spent on the net on knockdown and mortality (Figure 4). As previously mentioned, all mosquitoes survived contact with the control netting; however, the variation in contact time with the control net did not differ from the nets that were treated with insecticides (Table 3). Because of the high correlation between knock-down and the eventual survival, the figures for contact time and 24-hour survival look similar (Supplemental Figure 4). With deltamethrin only, the minimum contact time needed to cause knock-down was 0.4 seconds for a susceptible mosquito and 49.2 seconds for a VKPR mosquito; these mosquitoes died within 24 hours after exposure. For the deltamethrin + PBO netting, the minimum contact time causing knock-down and mortality was 0.12 seconds for a susceptible mosquito and 0.04 seconds for a VKPR mosquito. There was a positive correlation between the time resting on the net and knock-down rate (Figure 4). The contact time causing knock-down was generally shorter for deltamethrin + PBO than deltamethrin only. The maximum time that a susceptible mosquito was found unaffected after 1 hour (and 24 hours) was 40.3 seconds of contact with deltamethrin only and 64.7 seconds with deltamethrin + PBO. For the VKPR strain, these times were 16.2 and 0.4 seconds, respectively. 


\section{Chapter 7}

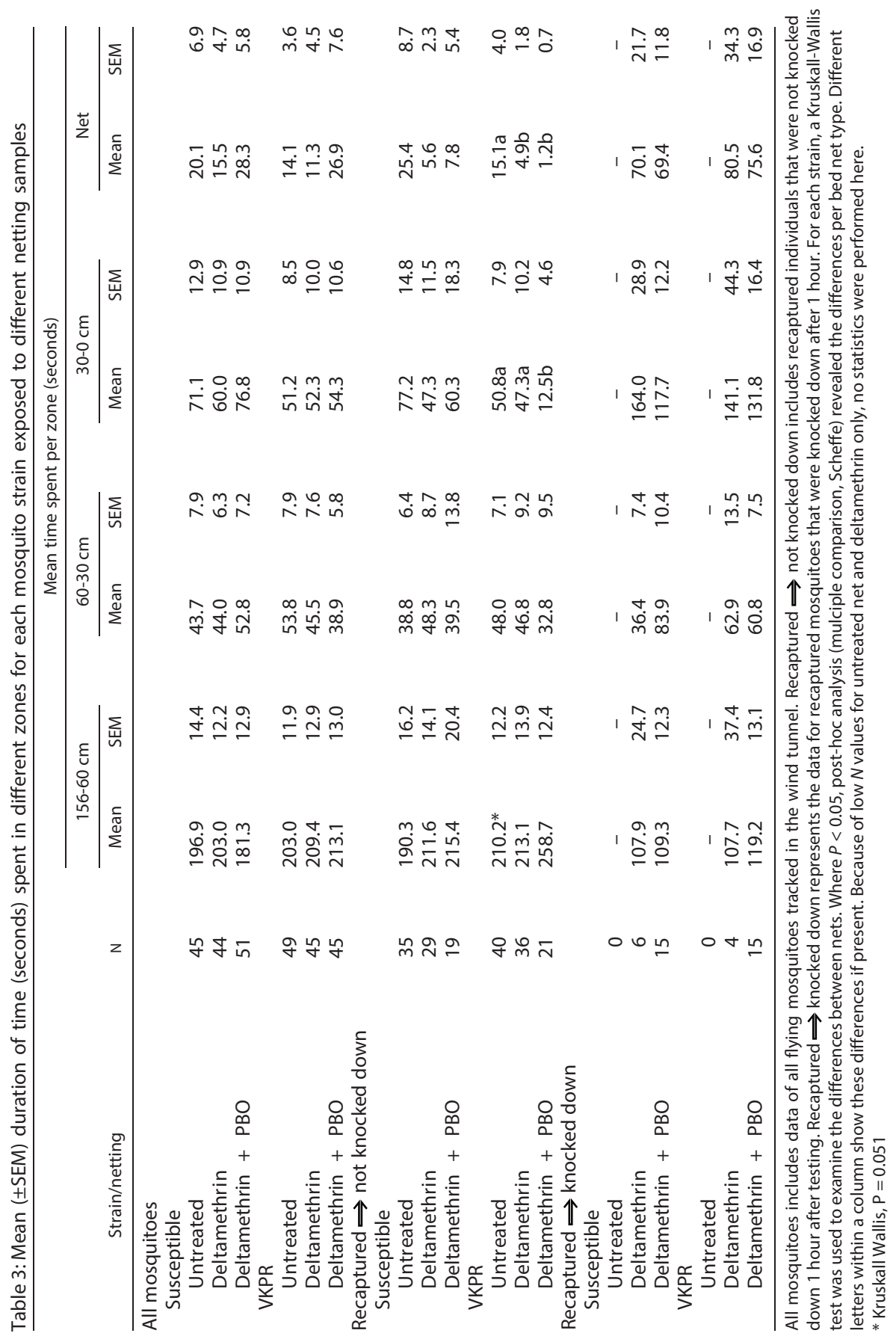




\section{Discussion}

The results revealed a great discrepancy between the WHO bioassays and wind tunnel bioassays, especially in terms of the eventual killing effect of ITNs. The insecticide-treated nettings in the WHO tube and cone bioassays all caused high knock-down and mortality in susceptible mosquitoes, showing the high degree of toxicity of these compounds for An. gambiae. Numerous studies have reported a repellent effect as well as a toxic effect of these compounds, and indeed, these effects are considered additional attributes for the protective effect of pyrethroids (Achee et al., 2009; Koudou et al., 2011). However, when these mosquitoes were exposed to both insecticidal treatments in the present study, in free flight, no repellent effect was observed. This result is evident by comparing the response and flight distribution patterns as well as the average time spent on the nettings. Only mosquitoes of the VKPR strain that survived the exposure to deltamethrin + PBO-treated nets expressed a mild repellent behaviour by spending more time at the downwind end of the wind tunnel. It seems, therefore, that (non-resistant) mosquitoes did not perceive deltamethrin and/or PBO within the range of $1.5 \mathrm{~m}$, the length of the wind tunnel.

Most studies on the effect of synthetic pyrethroids on mosquitoes have been conducted in experimental huts where the effect of the insecticides on the mosquitoes is indirectly measured by recording the proportion of mosquitoes that enter and/or exit the hut or the proportion of mosquitoes that are found dead (Carnevale et al., 1992; Darriet \& Chandre, 2011; Darriet et al., 1984; Hougard et al., 2003; Lines et al., 1987; Malima et al., 2009; Miller et al., 1991; N'Guessan et al., 2010). Mosquitoes that hover on and around a net acquire sufficient quantities of insecticide to be killed; however, experimental hut studies were not designed to observe flight and/or landing behaviours of mosquitoes on nets, and the behavioural effects of the nets on the mosquitoes are recorded by counting the fractions of mosquitoes that are left in the hut at the end of the exposure time, which are termed excitorepellent effects. A possible explanation for the widely reported excitorepellent effect with deltamethrintreated nets is that mosquitoes that enter a bedroom, attracted to human odours (Takken \& Knols, 1999), are prevented from bloodfeeding, because the host is protected by a bed net; hence, they remain hungry, which drives them out of the house. An increase in exiting behaviour in the presence of ITNs has been reported, where a proportion of the mosquito population succeeded to bloodfeed through manmade holes in the bed net (Corbel et al., 2010; Fane et al., 2012). However, parameters used in experimental hut studies that are expressed as excitorepellency as described in the work by Koudou et al. (2011), a study in which treated nets were intact, could be linked to the behavioural effects resulting from hunger. 


\section{Chapter 7}

The work by Lindsay et al. (1991), which did not find a repellent effect of the synthetic pyrethroid permethrin, suggest that the reported deterrent effect of synthetic pyrethroids is produced by the components of the emulsifiable concentrate used for impregnating the nets and not the insecticide itself. Similarly, Magesa et al. (1991) did not report an excitorepellent effect of pyrethroids. Because most compounds used for long-lasting manufacture of impregnated nets are inert, it is unlikely that such components cause excitorepellency. Kongmee et al. (2012) recently reported contact irritancy caused by deltamethrin in An. minimus Theobald and An. harrisoni Harbach and Manguin, but the irritant effect was less clear in the presence of host odours. Similar to our study, there was no spatial effect of deltamethrin on the behaviour of the mosquitoes. Additional analysis is required to determine whether host emanations can suppress irritant effects caused by the insecticide. In addition, it should be stressed that these findings were from experiments with unwashed new nets, and washed nets may give different results.

Our data are in line with the data reported in the work by Cooperband \& Allan (2009), which compared the excitorepellent effect of the synthetic pyrethroids bifenthrin, deltamethrin, and $\lambda$-cyhalothrin in a laboratory behavioural setting using the mosquito species Aedes aegypti (L.), An. quadrimaculatus Say, and Culex quinquefasciatus Say. The effect of the insecticides was observed from landing and resting behaviour on contact with insecticide-treated filter paper. No difference was observed between untreated and treated paper, suggesting that these three pyrethroids did not cause an excitorepellent effect. $C x$. quinquefasciatus, however, tended to avoid all three treated papers. Based on the heightened activity of some of the mosquitoes, it was suggested that the pyrethroids might affect locomotion behaviour, which was previously suggested by Schreck and Kline (1983). We have no information that this suggestion might be the case for An. gambiae.

The data from the WHO tube and cone bioassays indicated that the Wageningen An. gambiae strain (var. Suakoko) was susceptible to deltamethrin and that the VKPR strain was pyrethroid-resistant; mortalities with both formulations were $<80 \%$. In the wind tunnel, the only behavioural difference observed between mosquito strains was in latency time reaching the net. Additional studies are suggested to focus on the possible differences in physiological state between affected and surviving individuals. The unaffected group may have also spent less time in odour plumes containing host odours and deltamethrin than the mosquitoes that showed a knock-down response, which would be in line with the previously discussed results by Kongmee et al. (2012). The effect on knock-down and survival caused by deltamethrin netting was significantly less than that of deltamethrin + PBO netting, which could be a result 
of the higher dose of deltamethrin in the netting with PBO; however, it should be noted that, as the deltamethrin is incorporated into the polyethylene, less than $10 \%$ of the total dose $(4 \mathrm{~g} / \mathrm{kg}$ ) would be expected on the surface of the net and hence, available to the mosquito. In a coated net (such as the deltamethrin netting), much of the insecticide is on or near the surface. The presence of PBO may also affect the degree of knock-down and survival. Penetration, metabolic degradation, and interaction of insecticides with the site of action are known to influence knockdown (Ruigt, 1985). It has been suggested that synergists like PBO (which prevent detoxification of pyrethroids) have little effect on the knock-down phase of pyrethroid detoxification, while suppressing the recovery phase, which was shown in studies with Musca domestica and Triatoma infestans (Alzogaray \& Zerba, 1997; Sawicki, 1962). The increased efficacy of a deltamethrin + PBO-treated netting compared with a deltamethrin only-treated netting was shown by N'Guessan et al. (2010) using the WHO tunnel test assay with the VKPR strain. PBO is known to work by inhibiting metabolic enzymes (namely P450s and esterases) and enhance cuticular penetration of the insecticide into the insect (Ahmad et al., 2006; Bernard \& Philogene, 1993; Moores et al., 2005; Young et al., 2006). This effect on penetration occurs, because $\mathrm{PBO}$ also acts as a solvent to dissolve the insecticide and a surfactant on the waxy cuticle to increase the speed that the insecticide arrives at the target site, increasing efficacy of the insecticide, even with the presence of $k d r$ (Bingham et al., 2011). It is not known whether the effect observed in this study is caused by faster penetration of the insecticide through the cuticle because of the presence of PBO or the unknown presence of metabolic resistance in the VKPR strain, the different surface concentration, or the availability of deltamethrin in the deltamethrin + PBO netting.

The WHO tube and cone tests force mosquitoes into contact with the netting, and therefore, they pick up sufficient quantities of insecticide to cause knock-down and/or mortality within the specified exposure period. It is, therefore, an approved method to assess the bioefficacy of insecticide-impregnated materials. In the wind tunnel, by contrast, mosquitoes make a free flight and contact the netting without force when stimulated by the odour source present immediately behind the netting (Curtis, 1991; WHO, 2005). A large percentage of mosquitoes (86.5- 98.1\%) took off for upwind flight within 3 minutes after exposure to the nettings. If a spatial or contact repellent effect would have been present, it would have been expressed clearly by reduced contact of mosquitoes with the treated nets compared with the untreated net. Such effects were not seen, apart from the resistant mosquitoes that remained relatively unaffected by the treated nets. It would be interesting to investigate if this behaviour was related to the resistance mechanisms present in this mosquito strain. We conclude, therefore, that the treated nettings did not cause an excitorepellent 


\section{Chapter 7}

effect in contrast to other studies using different methodologies (Grieco et al., 2000; Koudou et al., 2011; Singh et al., 1989).

A lower efficacy of treated nettings was observed in the wind tunnel assay than the forced contact assays because the mosquitoes made less contact with the netting than in the WHO assays because of the free flight, which more closely resembles the natural situation than the $\mathrm{WHO}$ assays, where mosquitoes are in forced contact with the insecticides. Exposure to deltamethrin + PBO netting caused a significantly higher knock-down and mortality effect in the wind tunnel compared with deltamethrin alone, although we do not know whether this result was because of the different concentration of deltamethrin or the additional effect of PBO. It should be noted that under real-life conditions, a mosquito would have much longer than the 5-minute exposure time in this assay to make contact with the net; however, it is not known for how long a mosquito searches for a blood meal before abandoning this behaviour. To link the behavioural data with the WHO tests and the consequential health status of the tested mosquitoes, we kept the exposure time in the wind tunnel close to 3 minutes, adding 2 minutes for getting to the net and moving away from it.

The use of camera systems and automated tracking has made it possible to study differences in behaviour of various mosquito strains and species in both the laboratory and (semi) field settings (Spitzen et al., 2013). The wind tunnel assay is more sensitive to reveal behavioural effects than the current WHO assays and could be considered as an additional method useful for investigating spatial repellent effects of current and new insecticidal compounds (Achee et al., 2012). In the wind tunnel setup, in the presence of human host cues, it was found that both susceptible and resistant An. gambiae s.s. mosquitoes never spent as much as 3 minutes (cumulatively) on the test netting material in the absence of a bloodmeal. Although the 3-minute cone test remains a suitable method for investigating lethal and knockdown effects of pyrethroids, it does not provide any information regarding other potential behavioural responses that may be induced by exposure. Newly developed (non-pyrethroid) compounds may require longer contact times before a lethal dose will be picked up. Observational studies, such as the study presented here, can be used, for example, to validate appropriate exposure times for cone tests with new insecticides by investigating behavioural impacts of candidate compounds. The wind tunnel and video tracking assay could also be used to investigate how mosquitoes approach holes of different sizes in netting fabric as well as the optimum hole to insecticide content ratio or the optimum mesh size required for a netting fabric to physically prevent mosquito passage. 


\section{Conclusion}

Free-flight exposure of mosquitoes to deltamethrin-impregnated nets did not reveal any (excito-) repellent effects in susceptible and resistant mosquitoes caused by deltamethrin when using a video-recorded assay. Close-range behavioural assays can reveal important information concerning the way mosquitoes approach and contact insecticide-treated netting, which is particularly important when considering the design of non-pyrethroid insecticide-treated fabrics and their evaluation in the field.

\section{Acknowledgements}

The authors thank Frans van Aggelen, André Gidding and Léon Westerd for rearing of the mosquitoes and Julie-Anne Tangena for assistance with the World Health Organization assays. We are grateful to all blood donors for their donation.

\section{Supplemental videos 1-3 available at www.ajtmh.org}

http://www.ajtmh.org/content/journals/10.4269/ajtmh.13-0755\#supplementary_data

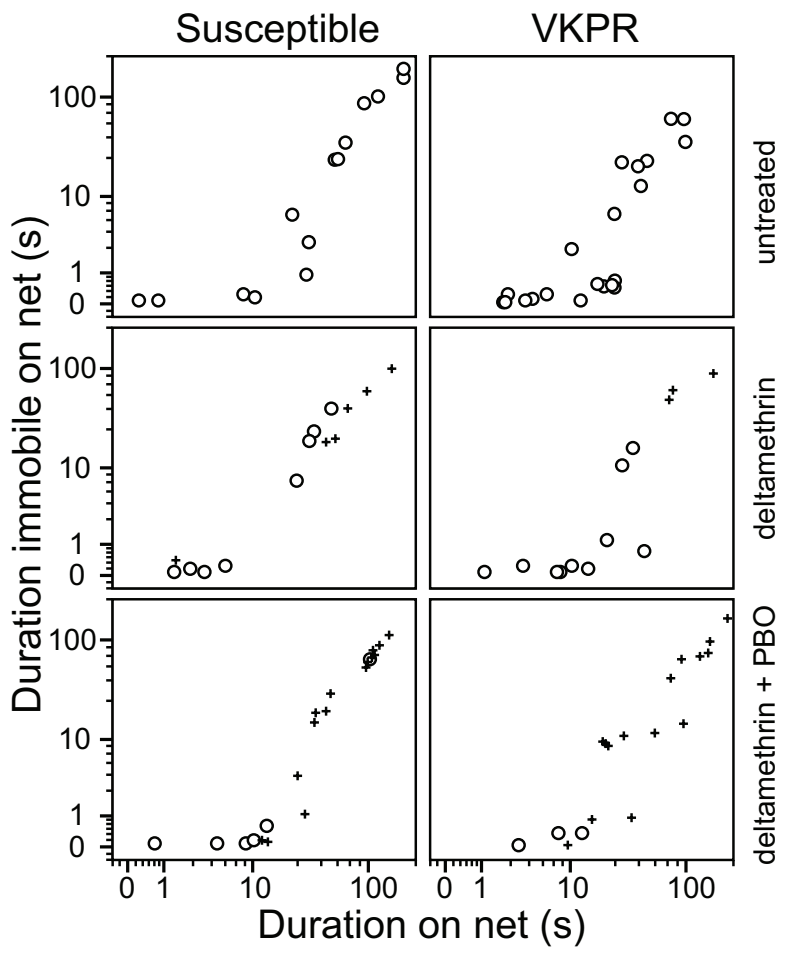

$24 \mathrm{hr}$ health status: o healthy, + died

Supplemental figure 4: $24 \mathrm{hr}$ health status (o healthy, + died) of both mosquito strains in relation to the duration spent on the net ( $x$ axis) and immobile on the net ( $y$ axis) in seconds. 
D<smiles>CI(CCCCCCC(C)(C)C)C(C)(C)C</smiles> 


\section{Chapter 8}

General Discussion 


\section{Chapter 8}

\section{Introduction}

Mosquitoes can be vectors of infectious diseases like dengue, chikungunya, filariasis and malaria, and this causes serious health problems across the world. Malaria, a disease caused by Plasmodium parasites and transmitted by mosquitoes of the genus Anopheles, was diagnosed for an estimated number of 216 million humans in 2016, with approximately 445,000 fatal cases (World Health Organization, 2017a). There has been an impressive decline in the number of malaria cases over the last 15 years thanks to rapid diagnosis and proper drug treatments, the use of longlasting-insecticide-treated nets (LLINs) and indoor residual spraying (IRS). However, existing control tools are under serious threat to lose their efficiency because of the rapid spread of physical and behavioural resistance (Hemingway, 2017). Therefore, an integrated approach is needed where existing and alternative vector control tools are combined (Alonso et al., 2017; Koenraadt \& Takken, 2018; World Health Organization, 2017b). Vector-control tools often aim to interrupt mosquitohost interactions. Besides the use of bed nets, this can be obtained by using odour-baited traps, push-pull systems, or making house improvements (Homan et al., 2016; Menger et al., 2016; Tusting et al., 2016). For the evaluation of existing tools, but especially for the innovation and successful implementation of alternative, sustainable tools it is necessary to understand mosquito behaviour in detail so that interventions can be made more precisely and effectively.

This thesis has addressed several aspects of mosquito flight behaviour that play a role during host seeking. The results contribute to our understanding of how the interaction of host cues, such as $\mathrm{CO}_{2}$, heat, and human odour affect their flight path and success in host finding. The development and application of an automated tracking system made it possible to analyse the approach towards host targets and to measure mosquito responses to interventions with bed nets, rather than measuring end points only. Detailed analysis of flight behaviour can be exploited to support the innovation of vector control tools and measure their effectiveness.

The main findings of the research described in this thesis are: 1) trap catches of female Anopheles gambiae s.s. can be enhanced by separation of the sources of the host-seeking stimuli carbon dioxide $\left(\mathrm{CO}_{2}\right)$ and human skin emanations, and deterrent effects of $\mathrm{CO}_{2}$ can be overcome by the simultaneous presence of skin emanations; 2) exposure of mosquitoes to human odour results in prolonged and highly convoluted flight tracks and the combination with heat is crucial to induce landings of host-seeking mosquitoes; 3 ) house-entering mosquitoes approach the eave of a house in a wide angle to the house at eave level, where the proportion that enters uninterruptedly (23\%) spent just a few seconds around the eave area; 4) the 
presence of insecticide-treated nets inside a house does not repel mosquitoes as measured by the number of house entries. However, the toxic effect of insecticidetreated nets seems to result in fewer unfed mosquitoes re-entering such a house; 5) free-flight exposure of mosquitoes to deltamethrin-treated nets in combination with human odour did not reveal any (excito-) repellent effect and resulted in lower mortality rates compared to standard World Health Organization (WHO) bioassays where contact with the treated material is enforced.

The results presented in this thesis contribute to the aim of the thesis to gain fundamental knowledge on how An. gambiae s.s. mosquitoes respond to a combination of host cues, in both laboratory- and semi-field settings. An automated 3D-tracking system was developed and implemented in different research setups, accomplishing the additional goal of this thesis to demonstrate that behaviouralecological studies on disease vectors can benefit from such tracking systems by providing detailed information on the approach of mosquitoes to different targets. The obtained knowledge can support the implementation and evaluation of vector control tools.

However, there is more to explore. This thesis is just a stepping stone to our understanding of mosquito behaviour and summarizes a selection of implementations for both fundamental and applied studies on malaria mosquitoes. It is promising to see that, in a time frame of less than 10 years, a variety of scientists around the world have adopted new techniques to (automatically) track the behaviours of mosquitoes and start to implement the results into applications that are tested under field conditions. In this final chapter, I will discuss the key findings and put them in perspective for future work on the behaviour of insect vectors.

\section{$\mathrm{CO}_{2}$, keystone in mosquito host-seeking behaviour}

The important effect of $\mathrm{CO}_{2}$ on the behaviour of blood-feeding arthropods has been recognized for decades (Gillies, 1980; Takken \& Knols, 2010). The sensory pathways activated upon exposure to $\mathrm{CO}_{2}$ are now clearly described after a series of studies on An. gambiae, Ae. aegypti and Culex quinquefasciatus (reviewed by Montell et al. (2016) and Ray (2015)). Interestingly, the capitate peg (cp) sensilla located on the maxillary palp contain three different neurons, of which the $\mathrm{cpA}$ neuron coexpresses three gustatory receptors (GRs) that seem highly conserved for all described mosquito species. This neuron contains receptors that, in addition to $\mathrm{CO}_{2}$, also respond to odorants that are associated with human skin emanations (Erdelyan et al., 2012; Lu et al., 2007; Syed \& Leal, 2007; Tauxe et al., 2013). Details on how $\mathrm{CO}_{2}$ activates and affects host-seeking responses have gradually been 


\section{Chapter 8}

added to the knowledge base. After the publication of our studies on the effect of human odours and positioning of the $\mathrm{CO}_{2}$ release point on trap catches (Chapter 3 ), several behavioural studies have investigated the mode of action of $\mathrm{CO}_{2}$ on hostseeking mosquitoes. Lacey et al. (2014) confirmed the superseding effect of skin odours over the response towards $\mathrm{CO}_{2}$ in Aedes aegypti mosquitoes. The multimodal integration of $\mathrm{CO}_{2}$ with other host cues is further described for both Ae. aegypti and An. gambiae (McMeniman et al., 2014; Webster et al., 2015). The detection and perception has been further unravelled for Ae. aegypti, An. gambiae and Cx. quinquefasciatus by Majeed et al. (2017). The activating role of $\mathrm{CO}_{2}$ in mosquitoes is clearly understood (Cardé \& Gibson, 2010; Gillies, 1980) but as reviewed for Ae. aegypti by Cardé (2015) (see Figure 1), the context of the stimulus presentation determines the behavioural outcome and this may explain the different findings from studies on the multi-modal interaction of host cues (van Breugel et al., 2015; Lacey et al., 2014; McMeniman et al., 2014). Accordingly, the relative attractiveness of $\mathrm{CO}_{2}$ and sequence of interactions with other host cues in more complex natural systems is less clear and besides that, seems to be species dependent (Majeed et al., 2017) (see Figure 1).

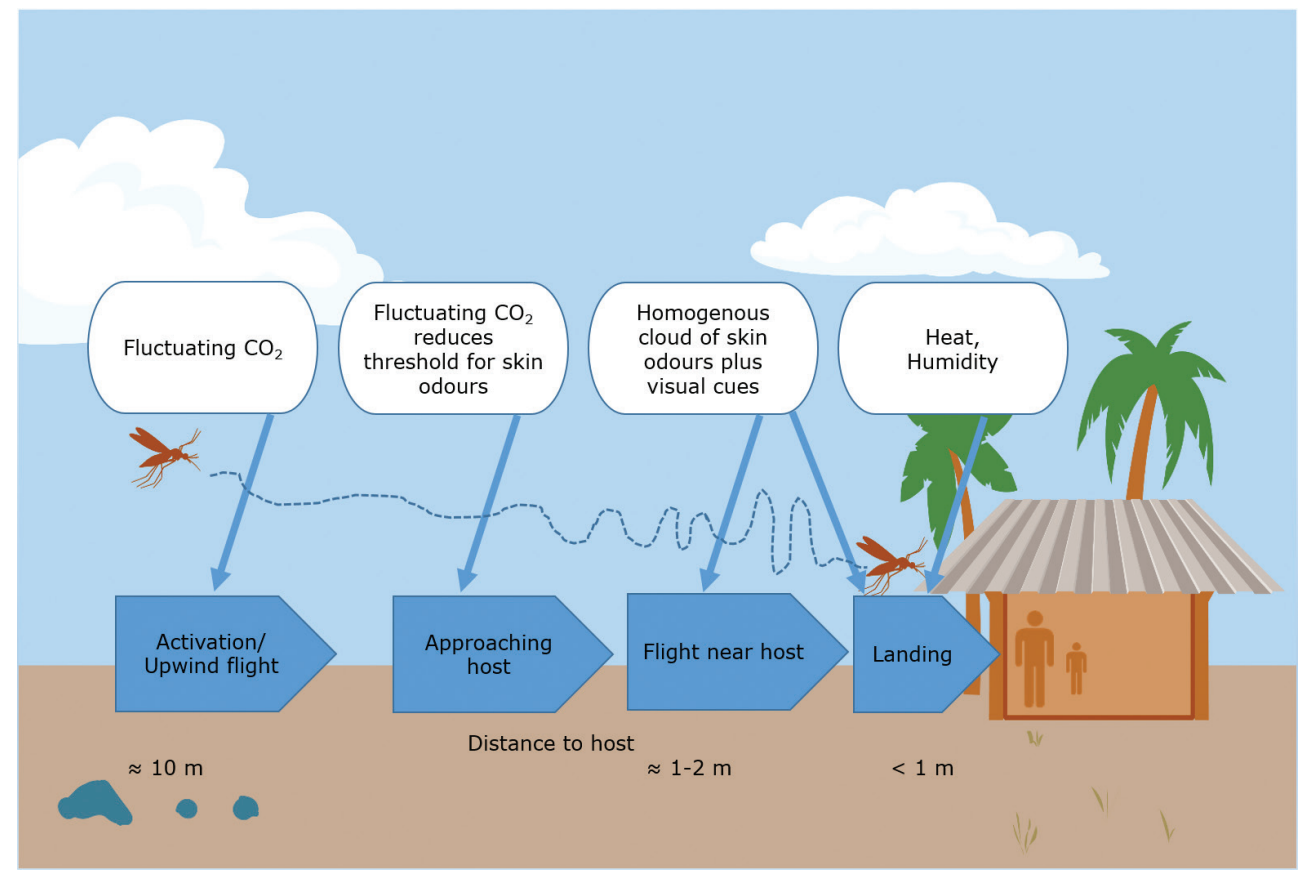

Figure 1. Presumed sequential encounter of host cues and navigational inputs. The figure was initially drawn for Aedes aegypti (adapted from Cardé (2015)), but in my opinion represents a generic overview of sequential interactions that occur during host-seeking of other mosquito species as well. The relative importance of each cue, the estimated distance from where it affects behaviour, depends on environmental conditions and differs between mosquito species. Infographic modified after Ties Blaauw, Communication Services, Wageningen University and Research. 
The majority of monitoring- and mosquito-control trapping tools rely on a sufficient supply of $\mathrm{CO}_{2}$. With that, large-scale applications in combination with long-lasting synthetic odour-baits form economical and logistical downsides (Homan et al., 2016; Loon et al., 2015; Smallegange et al., 2010b). Alternative cost-effective sources to either produce or substitute $\mathrm{CO}_{2}$ are required.

Behavioural studies on $\mathrm{CO}_{2}$ mimics can reveal to what extent the mimic affects the response to host cues, compared to exposure to $\mathrm{CO}_{2}$. For correct interpretations, these studies should incorporate the findings on the multimodal integration of other host cues as described above. Depending on the results, such studies should be brought to the semi-field for further evaluation. Responses to 2-butanone, a compound that can trigger activity of the $\mathrm{CO}_{2}$-sensitive neuron in several mosquito species, were very different between mosquito species when tested in the field (Mburu et al., 2017; Turner et al., 2011). Other studies that explored substitutions for $\mathrm{CO}_{2}$ reported minimal effects in the field compared to promising effects in the laboratory (cyclopentanone; Philippe-Janon et al. (2015) and acetone; Mboera et al., (2000c)). This can possibly be explained by the relative importance of other host cues that are present in the field and compete with the target compound.

\section{The need for heat and visual features}

The data presented in Chapter 4 demonstrate that adding heat to human skin emanations results in significantly more landings of mosquitoes near the source and both stimuli affected crosswind behaviours and flight speed, especially when mosquitoes had flown to within $30 \mathrm{~cm}$ from the source. McMeniman et al. (2014) and van Breugel et al. (2015) further unravelled the integration of different cues for Ae. aegypti including heat, $\mathrm{CO}_{2}$ and visual features (reviewed by Cardé (2015). Whereas $\mathrm{CO}_{2}$ was absent in our setup, McMeniman et al. (2014) demonstrated that $\mathrm{CO}_{2}$ detection causes mosquitoes to respond to heat and lactic acid in a $30 \mathrm{~cm}^{3}$ cage setup. The study by van Breugel et al. (2015) suggests that the attraction to heat by Ae. aegypti is independent of the presence of $\mathrm{CO}_{2}$, but can be allocated to visual cues that support the localization of heated objects. The difference between the two studies may also be explained by the distance at which the effects of the thermal targets are assessed. Up to $20 \mathrm{~cm}$ in the latter study, where it has to be noted that the heated elements in both Aedes studies were set at $37^{\circ} \mathrm{C}, 2-3$ degrees above skin temperature which may have affected the behavioural effects observed. Zermoglio et al. (2017) tested the orientation of Ae. aegypti mosquitoes to heat sources in the absence of other host cues to characterize discriminative abilities towards different heat sources that differed in size, temperature and the distance from the mosquito release point. When tested in a Y-tube choice setup, mosquitoes oriented towards 


\section{Chapter 8}

heat in the absence of other host cues, and orientation dominated towards short range $(16 \mathrm{~cm})$ heat $\left(34^{\circ} \mathrm{C}\right)$ sources.

The role of visual cues was neither examined in Chapter 4, nor by McMeniman et al. (2014). My wind-tunnel setup contained few contrasting objects to support visual feedback which could benefit efficient host-finding as suggested by Hawkes \& Gibson (2016). A recent field study by Hawkes et al. (2017) confirmed the importance of heat and visual cues in addition to odours and showed how a combination of human-associated cues can be exploited to considerably enhance trap catches of anophelines. These developments could replace the need for human-landing catches (HLC) as a monitoring tool, or to be used in vector control programmes. Careful evaluation of visual features or addition of heat sources should be performed since they can also be counterproductive if they evoke avoidance behaviour and prevent trap entry (Gillies \& Wilkes, 1982; Zermoglio et al., 2017). Ideally, the heat source does not require additional power supplies, or can be replaced by a low cost 'heat mimic' evoking comparable behavioural responses.

Overall, the conclusion on the role of the specific cues is very similar as discussed in the section on $\mathrm{CO}_{2}$. The quality of the different cues, together with the distance of the mosquito to these cues, affect the multimodal integration of the cues, resulting in a sequence of interactions that are involved in the process of host finding.

In Chapters 3 and 4, I have described how cues like $\mathrm{CO}_{2}$ and heat affect the flight behaviour and odour-source finding of mosquitoes in relation to the presence of skinrelated host odours. Both studies were performed in a confined space with controlled climate settings. In Chapter 5, where the house-entry behaviour of mosquitoes was analysed, host-seeking patterns are similar to those described for the 'odourexposed' mosquitoes in Chapter 4. While approaching the eave of a house, flight paths became more convoluted and mosquito flight speed was drastically reduced. However, besides the differences in complexity and environmental conditions of the experimental setup, mosquitoes that are about to enter a house are much further away from the target host-cues. Also, the house and the eave itself may function as visual stimuli (Bidlingmayer \& Hem, 1979, 1980). It was remarkable that mosquitoes spent very little time around the eave area before entering. They did not follow the wall to move upwards towards the eave, but approached at a wide angle, already at eave level when coming in view of the cameras. These chapters show the additional value of tracking flight behaviours above measuring end points only (Snow, 1987). Such knowledge can be further exploited by analysing the behaviours in relation to responses to odour-baited traps, push-pull systems, house improvements or the 
effectiveness of (insecticide-treated) bed nets (Hiscox et al., 2014; Menger et al., 2015; Parker et al., 2015; Tusting et al., 2015).

\section{Short time left for long-lasting-insecticide-treated nets?}

The decline in malaria incidences over the last 15 years, as mentioned in the introduction paragraph of this chapter, unfortunately came to a halt in 2016 (World Health Organization, 2017a). One of the main threats for a further decline in malaria cases is the rapid development of mosquito resistance to insecticides. As LLINs still protect humans from mosquito bites, the impact of insecticide resistance remains unclear but may also be difficult to measure (Huijben \& Paaijmans, 2017; Strode et al., 2014; World Health Organization, 2016b). Continued evaluation on the effectiveness of bed nets is required. The spread of insecticide resistance depends on the contact rate of mosquitoes to the specific compounds which they encounter in the environment, through the use of the same class of insecticides in agriculture, or via contact during host-seeking (Hemingway et al., 2016; Thomas et al., 2012). The contact of malaria mosquitoes with LLINs can be reduced if they would be repelled, which would enforce the mosquitoes to find another blood host (Birget \& Koella, 2015; Takken, 2002). This is an interesting trade off, since these mosquitoes will not be killed by the insecticide, but may still reproduce elsewhere (Lynch \& Boots, 2016). The level of repellency to insecticides is therefore of great interest to the industry, health institutes and vector ecologists. The literature reviewed in Chapters 2, 6 and 7 discloses a shift in insights on the level of repellency to bed nets, (mainly) based on experimental hut studies and more recent studies where the approach of mosquitoes to nets was observed. The general finding seems to be that nets are not as repellent as initially reported. However, it is important to note that this is not only based on more advanced research techniques, the production process of nets has also changed over the years. To date, manufacturers often incorporate the active ingredients inside the net fibres, and since then, there are few reports that demonstrate repellent effects of nets, apart from freshly-applied nets (Kongmee et al., 2012; Lindsay et al., 1991; Chapter 7). In theory, the addition of a spatial repellent to an effective insecticide can delay the evolution of resistance because fewer mosquitoes are exposed to the toxic compound, driving selection towards mosquitoes that are effectively repelled and manage to reproduce (Lynch \& Boots, 2016). The question here of course is: where do these mosquitoes take up a blood meal and which spatial repellent is strong enough to evoke the desired response? The use of repellents may have clear benefits for individual users but does not provide community protection (Huijben \& Paaijmans, 2017). It is unclear how mosquito populations respond to communitywide interventions using repellents. 


\section{Chapter 8}

Although bed nets still protect a large number of people from being bitten, behavioural changes of vectors have been reported through shifts in biting times (Moiroux et al., 2012) or host preference (Lefèvre et al., 2009). The relation with insecticide resistance and the effectiveness of LLINs cannot be measured with standard bioassays alone, where behavioural studies add information on the level of contact with the insecticides in more realistic settings (Kröner et al., 2016; Parker et al., 2017; Chapters 6,7). Such behavioural studies can also provide valuable information on the longevity, or level of protection, of nets (Sutcliffe et al., 2017). Ideally, individual mosquitoes must then be tracked over a longer period of time, both outside and inside houses during their host-seeking phase.

\section{Keeping track}

This thesis focused on behavioural aspects of mosquitoes during host-seeking. In Chapter 2 behavioural studies were highlighted that are relevant for other stages of the mosquito life cycle. Following the WHO Global Vector Control Response approach (Alonso et al., 2017; World Health Organization, 2017b) that aims for locally adapted, sustainable control measures, the knowledge obtained and reviewed in this thesis, can be further exploited for the development and implementation of such tools. A nice example is the previously mentioned field test by Hawkes et al. (2017) with the addition of a heat source to an odour baited trap.

Tracking techniques can be used to further 1) optimize intervention tools and 2) evaluate their effectiveness in the field. In addition to the alternative approaches (HELP) as part of an integrated vector management (IVM) strategy (Chapter 1), of which the urgency was recently stressed by Koenraadt \& Takken, (2018), there is a range of other intervention techniques on the rise. An overview is provided in Figure 2, adapted from Huijben and Paaijmans (2017). All the interventions mentioned to reduce transmission, have implications for the behavioural ecology of mosquitoes. Understanding the interactions between the implemented technique and the response of the mosquito can benefit the effectiveness of the tools. It is important to realize that successful implementation of these interventions needs to be fine-tuned for the different target species.

In collaboration with the Experimental Zoology group, Wageningen University and Research, I will continue to work on improved trapping techniques by including knowledge on the flight dynamics around counter-flow traps (Cribellier et al., submitted) and the flight responses to specific host cues, such as $\mathrm{CO}_{2}$ mimics. In the meantime, the effectiveness of house improvements is studied in (semi-) field settings in Africa (McCann et al., 2017). The information obtained by tracking 


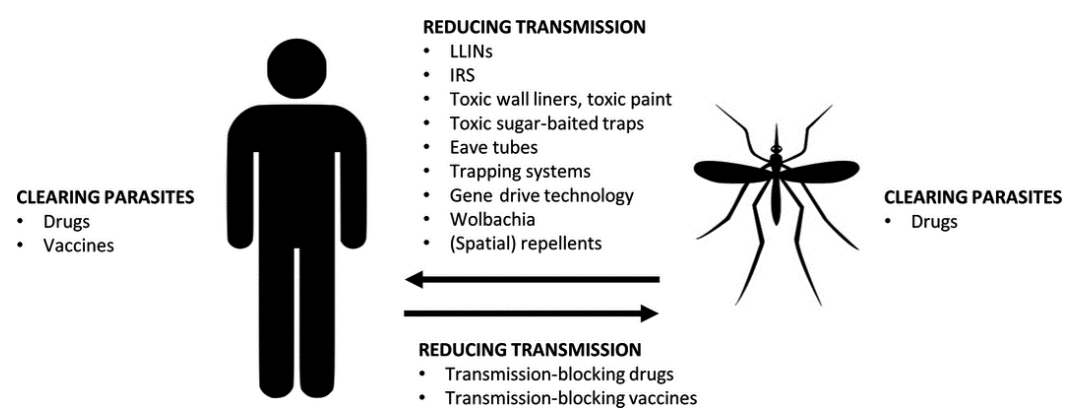

Figure 2. An overview of current and novel malaria control interventions (Huijben \& Paaijmans, 2017).

approaching mosquitoes can be used for the trade-off between complete or partly closure of (small) holes in new or renovated houses.

The developed research techniques and implemented tools to study and reduce the impact of vectors on human diseases are a product of many years of fundamental research and innovative programs. This know-how can also support the research of other 'vector-host' interactions or other multitrophic interactions. Studying host-plant resistance is an important aspect within sustainable pest management strategies against for e.g. thrips or aphids (Kloth et al., 2015; Thoen et al., 2016). Recently, automated tracking tools have been developed and implemented to monitor insect preference for certain varieties of ornamental- or crop plants. The studies complement end-point analysis and provide extra information on plant defence strategies against their attackers and the techniques can be applied for high-throughput screening of insect behaviour (Mouden et al., 2017).

\section{Concluding remarks}

This thesis provides answers to fundamental and applied aspects that play a role during host seeking of female mosquitoes. The research confirmed the crucial role of $\mathrm{CO}_{2}$ and its function as a key target for further exploration to manipulate or disrupt the host-seeking process. The development of automated tracking techniques has contributed to insights on the approach behaviour of mosquitoes rather than measuring end points only. By doing so, it was found that heat is vital for malaria mosquitoes to induce landings near an odour source, but that odour alone can evoke typical highly convoluted flight paths that were observed in wind tunnel studies as well as in the semi-field in Africa by studying mosquito house entry behaviour. The presence of insecticide-treated nets inside a house did not repel mosquitoes, however, their toxic effect seems to affect the number of mosquitoes that re-enter such houses. By recording the behaviour of mosquitoes exposed to insecticide-treated nets in a wind tunnel, their free-flight behaviour could be linked to 


\section{Chapter 8}

the mosquito health status and related to the contact time with the treated materials. The knowledge obtained on the behavioural responses of mosquitoes to host cues as described in this thesis indirectly affected vector control tool implementations in the field. For example in the development of the Suna trap, a $\mathrm{CO}_{2}$ release pipe was included that is separated from the attractive odour plume (Hiscox et al., 2014), the role of heat was exploited in the development of a repellent bioassay (Menger et al., 2014a) and a heat source was added to an odour-baited trap (Hawkes et al., 2017). The application of the tracking techniques developed in both laboratory and semi-field settings that lead to insights into house-entry behaviour and mosquito responses to bed nets, can be further exploited for the successful implementation of push-pull strategies and house-improvement operations. Finally, it will require an integrated vector management approach, with a cocktail of effective tools, to successfully combat one of human's main natural enemy.

\section{Acknowledgements}

I would like to thank Willem Takken and Marcel Dicke for comments on a previous version of this chapter. 
General Discussion 


$$
8
$$


References 
Achee, N. L., Bangs, M. J., Farlow, R., Killeen, G. F., Lindsay, S., Logan, J. G., Moore, S. J., Rowland, M., Sweeney, K., Torr, S. J., Zwiebel, L., \& Grieco, J. P. (2012). Spatial repellents: from discovery and development to evidence-based validation. Malaria Journal, 11, 164.

Achee, N. L., Sardelis, M. R., Dusfour, I., Chauhan, K. R., \& Grieco, J. P. (2009). Characterization of spatial repellent, contact irritant, and toxicant chemical actions of standard vector control compounds. Journal of the American Mosquito Control Association, 25(2), 156-167.

Achinko, D., Thailayil, J., Paton, D., Mireji, P. O., Talesa, V., Masiga, D., \& Catteruccia, F. (2016). Swarming and mating activity of Anopheles gambiae mosquitoes in semi-field enclosures. Medical and Veterinary Entomology, 30(1), 14-20.

Afify, A., \& Galizia, C. G. (2015). Chemosensory cues for mosquito oviposition site selection. Journal of Medical Entomology, 52(2), 120-130.

Afify, A., Horlacher, B., Roller, J., \& Galizia, C. G. (2014). Different repellents for Aedes aegypti against blood-feeding and oviposition. PLoS One, 9(7), e103765.

Ahmad, M., Denholm, I., \& Bromilow, R. H. (2006). Delayed cuticular penetration and enhanced metabolism of deltamethrin in pyrethroid-resistant strains of Helicoverpa armigera from China and Pakistan. Pest Management Science, 62(9), 805-810.

Alonso, P. L., Engels, D., \& Reeder, J. (2017). Renewed push to strengthen vector control globally. The Lancet, 389(10086), 2270-2271.

Aly, A. S. I., Vaughan, A. M., \& Kappe, S. H. I. (2009). Malaria parasite development in the mosquito and infection of the mammalian host Annual Review of Microbiology (Vol. 63, pp. 195-221).

Alzogaray, R. A., \& Zerba, E. N. (1997). Incoordination, paralysis and recovery after pyrethroid treatment on nymphs III of Triatoma infestans (Hemiptera: Reduviidae). Memorias do Instituto Oswaldo Cruz, 92, 431-435.

Andriessen, R., Snetselaar, J., Suer, R. A., Osinga, A. J., Deschietere, J., \& Lyimo, I. N. (2015). Electrostatic coating enhances bioavailability of insecticides and breaks pyrethroid resistance in mosquitoes. Proceedings of the National Academy of Sciences, 112(39), 12081-12086.

Angarita-Jaimes, N. C., Parker, J. E., Abe, M., Mashauri, F., Martine, J., Towers, C. E., McCall, P. J., \& Towers, D. P. (2016). A novel video-tracking system to quantify the behaviour of nocturnal mosquitoes attacking human hosts in the field. Journal of The Royal Society Interface, 13(117), 20150974.

Balestrino, F., Iyaloo, D. P., Elahee, K. B., Bheecarry, A., Campedelli, F., Carrieri, M., \& Bellini, R. (2016). A sound trap for Aedes albopictus (Skuse) male surveillance: Response analysis to acoustic and visual stimuli. Acta Tropica, 164, 448-454.

Balkenius, A., \& Dacke, M. (2010). Flight behaviour of the hawkmoth Manduca sexta towards unimodal and multimodal targets. Journal of Experimental Biology, 213(Pt 21), 3741-3747.

Beehler, J. W., Millar, J. G., \& Mulla, M. S. (1993). Synergism between chemical attractants and visual cues influencing oviposition of the mosquito, Culex quinquefasciatus (Diptera: Culicidae). Journal of Chemical Ecology, 19(4), 635-644.

Benelli, G. (2015a). The best time to have sex: mating behaviour and effect of daylight time on male sexual competitiveness in the Asian tiger mosquito, Aedes albopictus (Diptera: Culicidae). Parasitology Research, 114(3), 887-894.

Benelli, G. (2015b). Research in mosquito control: current challenges for a brighter future. Parasitology Research, 114(8), 2801-2805.

Benelli, G., Jeffries, C. L., \& Walker, T. (2016). Biological control of mosquito vectors: Past, present, and future. Insects, 7(4), 52.

Benelli, G., \& Mehlhorn, H. (2016). Declining malaria, rising of dengue and Zika virus: insights for mosquito vector control. Parasitology Research, 115(5), 1747-1754.

Bernard, C. B., \& Philogene, B. J. R. (1993). Insecticide synergists: Role, importance, and perspectives. Journal of Toxicology and Environmental Health, 38(2), 199-223. 


\section{References}

Bernáth, B., Anstett, V., \& Guerin, P. M. (2016). Anopheles gambiae females readily learn to associate complex visual cues with the quality of sugar sources. Journal of Insect Physiology, 95, 8-16.

Berry, W. J., Rowley, W. A., \& Christensen, B. M. (1988). Spontaneous flight activity of Aedes trivittatus infected with Dirofilaria immitis. Journal of Parasitology, 74(6), 970-974.

Bhasin, A., Mordue, A. J., \& Mordue, W. (2001). Field studies on efficacy of host odour baits for the biting midge Culicoides impunctatus in Scotland. Medical and Veterinary Entomology(15), 147-156.

Bhatt, S., Weiss, D., Cameron, E., Bisanzio, D., Mappin, B., \& Dalrymple, U. (2015). The effect of malaria control on Plasmodium falciparum in Africa between 2000 and 2015. Nature, 526, 207-211.

Bidlingmayer, W. L., \& Hem, D. G. (1979). Mosquito (Dipteral Culicidae) flight behaviour near conspicuous objects. Bulletin of Entomological Research, 69(4), 691-700.

Bidlingmayer, W. L., \& Hem, D. G. (1980). The range of visual attraction and the effect of competitive visual attractants upon mosquito (Diptera: Culicidae) flight. Bulletin of Entomological Research, 70, 321-342.

Billingsley, P. F., Hodivala, K. J., Winger, L. A., \& Sinden, R. E. (1991). Detection of mature malaria infections in live mosquitoes. Transactions of the Royal Society of Tropical Medicine and Hygiene, 85, 450-453.

Bingham, G., Strode, C., Tran, L., Khoa, P. T., \& Jamet, H. P. (2011). Can piperonyl butoxide enhance the efficacy of pyrethroids against pyrethroid-resistant Aedes aegypti? Tropical Medicine and International Health, 16(4), 492-500.

Binka, F. N., Kubale, A., Adjuik, M., Williams, L. A., Lengeler, C., Maude, G. H., Armah, G. E., Kajihara, B., Adiamah, J. H., \& Smith, P. G. (1996). Impact of permethrin impregnated bednets on child mortality in Kassena-Nankana district, Ghana: A randomized controlled trial. Tropical Medicine and International Health, 1(2), 147-154.

Birget, P. L. G., \& Koella, J. C. (2015). An epidemiological model of the effects of insecticide-treated bed nets on malaria transmission. PLoS One, 10(12), e0144173.

Blanford, S., Chan, B. H., Jenkins, N., Sim, D., Turner, R. J., Read, A. F., \& Thomas, M. B. (2005). Fungal pathogen reduces potential for malaria transmission. Science, 308(5728), 1638-1641.

Blaustein, L., \& Chase, J. M. (2007). Interactions between mosquito larvae and species that share the same trophic level Annual Review of Entomology (Vol. 52, pp. 489-507).

Bodin, A., Vinauger, C., \& Lazzari, C. R. (2009). Behavioural and physiological state dependency of host seeking in the bloodsucking insect Rhodnius prolixus. Journal of Experimental Biology, 212, 2386-2393.

Bogh, C., Pedersen, E. M., Mukoko, D. A., \& Ouma, J. H. (1998). Permethrin-impregnated bednet effects on resting and feeding behaviour of lymphatic filariasis vector mosquitoes in Kenya. Medical and Veterinary Entomology, 12(1), 52-59.

Bomphrey, R. J., Nakata, T., Phillips, N., \& Walker, S. M. (2017). Smart wing rotation and trailing-edge vortices enable high frequency mosquito flight. Nature, 544(7648), 92-95.

Boyce, R., Lenhart, A., Kroeger, A., Velayudhan, R., Roberts, B., \& Horstick, O. (2013). Bacillus thuringiensis israelensis (Bti) for the control of dengue vectors: Systematic literature review. Tropical Medicine and International Health, 18(5), 564-577.

Bradley, J., Hergott, D., Garcia, G., Lines, J., Cook, J., Slotman, M. A., Phiri, W. P., Schwabe, C., \& Kleinschmidt, I. (2016). A cluster randomized trial comparing deltamethrin and bendiocarb as insecticides for indoor residual spraying to control malaria on Bioko Island, Equatorial Guinea. Malaria Journal, 15(1), 378.

Braks, M. A. H., Cooperband, M. F., \& Cardé, R. T. (2005). 3-D flight track analyses of Culex mosquito orientation to oviposition-related odors. Paper presented at Measuring Behavior, 5th International Conference on Methods and Techniques in Behavioral Research, Wageningen, The Netherlands. 
Briegel, H., \& Horler, E. (1993). Multiple blood meals as a reproductive strategy in Anopheles (Diptera: Culicidae). Journal of Medical Entomology, 30, 975-985.

Briët, O. J., Penny, M. A., Hardy, D., Awolola, T. S., van Bortel, W., Corbel, V., Dabiré, R. K., Etang, J., Koudou, B. G., Tungu, P. K., \& Chitnis, N. (2013). Effects of pyrethroid resistance on the cost effectiveness of a mass distribution of long-lasting insecticidal nets: a modelling study. Malaria Journal, 12(1), 77.

Brogdon, W. G. (1994). Measurement of flight tone differences between female Aedes aegypti and $A$. albopictus (Diptera: Culicidae). Journal of Medical Entomology, 31(5), 700-703.

Brogdon, W. G. (1998). Measurement of flight tone differentiates among members of the Anopheles gambiae species complex (Diptera:Culicidae). Journal of Medical Entomology, 35(5), 681-684.

Brown, D. (2014). Getting started with Tracker, https://www.youtube.com/watch?v=La3H7JywgX0.

Budick, S. A., \& Dickinson, M. H. (2006). Free-flight responses of Drosophila melanogaster to attractive odors. Journal of Experimental Biology, 209(15), 3001-3017.

Busula, A. O., Bousema, T., Mweresa, C. K., Masiga, D., Logan, J. G., Sauerwein, R. W., Verhulst, N. O., Takken, W., \& de Boer, J. G. (2017). Gametocytemia and attractiveness of plasmodium falciparum-infected kenyan children to Anopheles gambiae mosquitoes. The Journal of Infectious Diseases, 216(3), 291-295.

Butail, S., Manoukis, N., Diallo, M., Ribeiro, J. M., Lehmann, T., \& Paley, D. A. (2012). Reconstructing the flight kinematics of swarming and mating in wild mosquitoes. Journal of The Royal Society Interface, 9(75), 2624-2638.

Butail, S., Manoukis, N. C., Diallo, M., Ribeiro, J. M. C., \& Paley, D. A. (2013). The dance of male Anopheles gambiae in wild mating swarms. Journal of Medical Entomology, 50(3), 552-559.

Butler, J. F. (2007). Use of olfactometers for determining attractants and repellents. In M. Debboun, S. P. Frances, D. Strickman, \& F. L. Boca Raton (Eds.), Insect repellents: principles, methods and uses. Boca Raton, Florida, USA: Francis Group.

Caminade, C., Kovats, S., Rocklov, J., Tompkins, A. M., Morse, A. P., Colón-González, F. J., Stenlund, H., Martens, P., \& Lloyd, S. J. (2014). Impact of climate change on global malaria distribution. Proceedings of the National Academy of Sciences, 111(9), 3286-3291.

Caprio, M. A., Huang, J. X., Faver, M. K., \& Moore, A. (2001). Characterization of male and female wingbeat frequencies in the Anopheles quadrimaculatus complex in Mississippi. Journal of the American Mosquito Control Association, 17(3), 186-189.

Cardé, R. T. (2015). Multi-Cue Integration: How Female Mosquitoes Locate a Human Host. Current Biology, 25(18), R793-R795.

Cardé, R. T., \& Bell, W. J. (Eds.). (1995). Chemical Ecology of Insects 2. New York: Chapman and Hall.

Cardé, R. T., \& Gibson, G. (2010). Host finding by female mosquitoes: mechanisms of orientation to host odours and other cues. In W. Takken \& B. G. J. Knols (Eds.), Olfaction in vector-host interactions (pp. 115-141). Wageningen: Wageningen Academic Publishers.

Cardé, R. T., \& Willis, M. A. (2008). Navigational strategies used by insects to find distant, wind-borne sources of odor. Journal of Chemical Ecology, 34, 854-866.

Carnevale, P., Bitsindou, P., Diomande, L., \& Robert, V. (1992). Insecticide impregnation can restore the efficiency of torn bed nets and reduce man-vector contact in malaria endemic areas. Transactions of the Royal Society of Tropical Medicine and Hygiene, 86, 362-364.

Carvalho, D. O., McKemey, A. R., Garziera, L., Lacroix, R., Donnelly, C. A., Alphey, L., Malavasi, A., \& Capurro, M. L. (2015). Suppression of a field population of Aedes aegypti in Brazil by sustained release of transgenic male mosquitoes. PLoS Neglected Tropical Diseases, 9(7), e0003864.

Cator, L. J., George, J., Blanford, S., Murdock, C. C., Baker, T. C., Read, A. F., \& Thomas, M. B. (2013). 'Manipulation' without the parasite: Altered feeding behaviour of mosquitoes is not dependent on infection with malaria parasites. Proceedings of the Royal Society B: Biological Sciences, 280(1763), 20130803. 


\section{References}

Cator, L. J., Lynch, P. A., Read, A. F., \& Thomas, M. B. (2012). Do malaria parasites manipulate mosquitoes? Trends in Parasitology, 28(11), 467-470.

Cator, L. J., Ng'Habi, K. R., Hoy, R. R., \& Harrington, L. C. (2010). Sizing up a mate: Variation in production and response to acoustic signals in Anopheles gambiae. Behavioral Ecology, 21(5), 1033-1039.

Chambers, E. W., Hapairai, L., Peel, B. A., Bossin, H., \& Dobson, S. L. (2011). Male mating competitiveness of a Wolbachia-introgressed Aedes polynesiensis strain under semi-field conditions. PLoS Neglected Tropical Diseases, 5(8), e1271.

Chapman, J. W., Drake, V. A., \& Reynolds, D. R. (2011). Recent insights from radar studies of insect flight Annual Review of Entomology (Vol. 56, pp. 337-356).

Charlwood, D. J. (1974). Infrared T.V. for watching mosquito behaviour in the 'dark'. Transactions of the Royal Society of Tropical Medicine and Hygiene, 68(4), 364.

Choi, D. B., Grieco, J. P., Apperson, C. S., Schal, C., Ponnusamy, L., Wesson, D. M., \& Achee, N. L. (2016). Effect of spatial repellent exposure on dengue vector attraction to oviposition sites. PLoS Neglected Tropical Diseases, 10(7), e0004850.

Clements, A. N. (1992). The Biology of Mosquitoes (Vol. I). London: Chapman \& Hall.

Clements, A. N. (1999). The Biology of Mosquitoes (Vol. II). Wallingford, UK: CABI Publishers.

Coetzee, M., Hunt, R. H., Wilkerson, R., Torre, A. D., Coulibaly, M. B., \& Besansky, N. J. (2013). Anopheles coluzzii and Anopheles amharicus, new members of the Anopheles gambiae complex. Zootaxa, 3619(3), 246-274.

Cook, S. M., Khan, Z. R., \& Pickett, J. A. (2007). The use of push-pull strategies in integrated pest management. Annual Review of Entomology, 52, 375-400.

Cooperband, M. F., \& Allan, S. A. (2009). Effects of different pyrethroids on landing behavior of female Aedes aegypti, Anopheles quadrimaculatus, and Culex quinquefasciatus mosquitoes (Diptera: Culicidae). Journal of Medical Entomology, 46(2), 292-306.

Cooperband, M. F., \& Cardé, R. T. (2006). Orientation of Culex mosquitoes to carbon dioxide-baited traps: flight manoeuvres and trapping efficiency. Medical and Veterinary Entomology, 20(1), 11-26.

Corbel, V., Chabi, J., Dabire, R. K., Etang, J., Nwane, P., Pigeon, O., Akogbeto, M., \& Hougard, J. M. (2010). Field efficacy of a new mosaic long-lasting mosquito net (PermaNet (R) 3.0) against pyrethroid-resistant malaria vectors: a multi centre study in Western and Central Africa. Malaria Journal, 9, 113.

Costantini, C., Gibson, G., Sagnon, N., Torre, A. d., Brady, J., \& Coluzzi, M. (1996). Mosquito responses to carbon dioxide in a West African Sudan savanna village. Medical and Veterinary Entomology, 10, 220-227.

Costantini, C., Sagnon, N., della Torre, A., \& Coluzzi, M. (1999). Mosquito behavioural aspects of vector-human interactions in the Anopheles gambiae complex. Parassitologia, 41, 209-217.

Cummins, B., Cortez, R., Foppa, I. M., Walbeck, J., \& Hyman, J. M. (2012). A spatial model of mosquito host-seeking behavior. PLoS Computational Biology, 8(5), e1002500.

Curtis, C. F. (Ed.). (1991). Control of Disease Vectors in the Community. (pp. 233) London: Wolfe Publishing Ltd.

Dambach, P., Louis, V. R., Kaiser, A., Ouedraogo, S., Sié, A., Sauerborn, R., \& Becker, N. (2014). Efficacy of Bacillus thuringiensis var. israelensis against malaria mosquitoes in northwestern Burkina Faso. Parasites \& Vectors, 7(1), 371.

Darriet, F., \& Chandre, F. (2011). Combining piperonyl butoxide and dinotefuran restores the efficacy of deltamethrin mosquito nets against resistant Anopheles gambiae (Diptera: Culicidae). Journal of Medical Entomology, 48(4), 952-955. 
Darriet, F., Robert, V., Tho Vien, N., \& Carnevale, P. (1984). Evaluation of the efficacy of Permethrinimpregnated intact and perforated mosquito nets against vectors of malaria. WHO Bulletin, WHONBC, 84(899), 19pp.

David, C. T. (1986). Mechanisms of directional flight in wind. In T. L. Payne, M. C. Birch, \& C. E. J. Kennedy (Eds.), Mechanisms in Insect Olfaction (pp. 49-75). Oxford: Oxford University Press.

David, C. T., \& Birch, M. C. (1989). Pheromones and insect behaviour. In: Insect Pheromones in Plant Protection, Jutsum \& Gordon, 17-35.

Davies, E. R. (2012). Computer and Machine Vision. 4. London: Academic Press.

Davis, E. E. (1984). Regulation if sensitivity in the peripheral chemoreceptor systems for host-seeking behaviour by a haemolymph-borne factor in Aedes aegypti. Journal of Insect Physiology, 30, 179-183.

de Groot, J. H., \& van Leeuwen, J. L. (2002). Estimation of the longitudinal axis of line symmetrical soft bodies by stereophotogrammetry. Journal of Biomechanics, 35(6), 823-827.

de Jong, R. \& Knols, B. G. J. (1995). Selection of biting sites on man by two malaria mosquito species. Experientia , 51, 80-84.

de Moraes, C. M., Stanczyk, N. M., Betz, H. S., Pulido, H., Sim, D. G., Read, A. F., \& Mescher, M. C. (2014). Malaria-induced changes in host odors enhance mosquito attraction. Proceedings of the National Academy of Sciences, 111(30), 11079-11084.

Dekker, T., \& Cardé, R. T. (2011). Moment-to-moment flight manoeuvres of the female yellow fever mosquito ( Aedes aegypti L.) in response to plumes of carbon dioxide and human skin odour. Journal of Experimental Biology, 214(20), 3480-3494.

Dekker, T., Geier, M., \& Cardé, R. T. (2005). Carbon dioxide instantly sensitizes female yellow fever mosquitoes to human skin odours. Journal of Experimental Biology, 208(15), 2963-2972.

Dekker, T., \& Takken, W. (1998). Differential responses of mosquito sibling species Anopheles arabiensis and An. quadriannulatus to carbon dioxide , a man or a calf. Medical and Veterinary Entomology, 12, 136-140.

Dekker, T., Takken, W., \& Cardé, R. T. (2001). Structure of host-odour plumes influences catch of Anopheles gambiae s.s. and Aedes aegypti in a dual-choice olfactomer. Physiological Entomology, 26, 124-134.

Diabate, A., Baldet, T., Chandre, F., Akoobeto, M., Guiguemde, T. R., Darriet, F., Brengues, C., Guillet, P., Hemingway, J., Small, G. J., \& Hougard, J. M. (2002). The role of agricultural use of insecticides in resistance to pyrethroids in Anopheles gambiae s.I. in Burkina Faso. American Journal of Tropical Medicine and Hygiene, 67(6), 617-622.

Diabate, A., \& Tripet, F. (2015). Targeting male mosquito mating behaviour for malaria control. Parasites \& Vectors, 8, 347.

Dickerson, A. K., Shankles, P. G., Berry Jr, B. E., \& Hu, D. L. (2015). Fog and dense gas disrupt mosquito flight due to increased aerodynamic drag on halteres. Journal of Fluids and Structures, 55, 451-462.

Dickerson, A. K., Shankles, P. G., Madhavan, N. M., \& Hu, D. L. (2012). Mosquitoes survive raindrop collisions by virtue of their low mass. Proceedings of the National Academy of Sciences, 109(25), 9822-9827.

Downes, J. A. (1969). The swarming and mating flight of diptera. Annual Review of Entomology, 14, 271-298.

Dugassa, S., Lindh, J. M., Torr, S. J., Lindsay, S. W., \& Fillinger, U. (2014). Evaluation of the influence of electric nets on the behaviour of oviposition site seeking Anopheles gambiae s.s. Parasites \& Vectors, 7(1), 272.

Durnez, L., Mao, S., Denis, L., Roelants, P., Sochantha, T., \& Coosemans, M. (2013). Outdoor malaria transmission in forested villages of Cambodia. Malaria Journal, 12, 329. 
Eneh, L. K., Okal, M. N., Borg-Karlson, A. K., Fillinger, U., \& Lindh, J. M. (2016). Gravid Anopheles gambiae sensu stricto avoid ovipositing in Bermuda grass hay infusion and it's volatiles in two choice egg-count bioassays. Malaria Journal, 15(1), 276.

Erdelyan, C. N. G., Mahood, T. H., Bader, T. S. Y., \& Whyard, S. (2012). Functional validation of the carbon dioxide receptor genes in Aedes aegypti mosquitoes using RNA interference. Insect Molecular Biology, 21(1), 119-127.

Essen, P. H. A. v., Kemme, J. A., Ritchie, S. A., \& Kay, B. H. (1994). Differential responses of Aedes and Culex mosquitoes to octenol or light in combination with carbon dioxide in Queensland, Australia. Medical and Veterinary Entomology, 8, 63-67.

Evans, O., Caragata, E. P., McMeniman, C. J., Woolfit, M., Green, D. C., Williams, C. R., Franklin, C. E., O'Neill, S. L., \& McGraw, E. A. (2009). Increased locomotor activity and metabolism of Aedes aegypti infected with a lifeshortening strain of Wolbachia pipientis. Journal of Experimental Biology, 212(10), 1436-1441.

Faiman, R., Kirstein, O., Moncaz, A., Guetta, H., \& Warburg, A. (2011). Studies on the flight patterns of foraging sand flies. Acta Tropica, 120(1/2), 110-114.

Fane, M., Cisse, O., Traore, C. S. F., \& Sabatier, P. (2012). Anopheles gambiae resistance to pyrethroid-treated nets in cotton versus rice areas in Mali. Acta Tropica, 122(1), 1-6.

Farjana, T., Tuno, N., \& Higa, Y. (2011). Effects of temperature and diet on development and interspecies competition in Aedes aegypti and Aedes albopictus. Medical and Veterinary Entomology, 26(2), 210-217.

Favret, C., \& Sieracki, J. M. (2016). Machine vision automated species identification scaled towards production levels. Systematic Entomology, 41(1), 133-143.

Fillinger, U., Knols, B. G. J., \& Becker, N. (2003). Efficacy and efficiency of new Bacillus thuringiensis var. israelensis and Bacillus sphaericus formulations against Afrotropical anophelines in Western Kenya. Tropical Medicine and International Health, 8(1), 37-47.

Foster, W. A. (1995). Mosquito sugar feeding and reproductive energetics. Annual Review of Entomology, 40, 443-447.

Foster, W. A., \& Takken, W. (2004). Nectar-related vs. human-related volatiles: behavioural response and choice by female and male Anopheles gambiae (Diptera: culicidae) between emergence and first feeding. Bulletin of Entomological Research, 94.

Fry, S. N., Bichsel, M., Müller, P., \& Robert, D. (2000). Tracking of flying insects using pan-tilt cameras. Journal of Neuroscience Methods, 101(1), 59-67.

Fu, S., Biwole, P. H., \& Mathis, C. (2015). Particle Tracking Velocimetry for indoor airflow field: A review. Building and Environment, 87, 34-44.

Gatton, M. L., Chitnis, N., Churcher, T., Donnelly, M. J., Ghani, A. C., \& Godfray, H. C. J. (2013). The importance of mosquito behavioural adaptations to malaria control in Africa. Evolution, 67(4), 1218-1230.

George, J., Blanford, S., Domingue, M. J., Thomas, M. B., Read, A. F., \& Baker, T. C. (2011). Reduction in host-finding behaviour in fungus-infected mosquitoes is correlated with reduction in olfactory receptor neuron responsiveness. Malaria Journal, 10, 219.

Gibson, G. (1995). A behavioural test of the sensitivity of a nocturnal mosquito, Anopheles gambiae, to dim white, red and infra-red light. Physiological Entomology, 20(3), 224-228.

Gibson, G., \& Brady, J. (1988). Flight behaviour of tsetse flies in host odour plumes: the initial response to leaving or entering odour. Physiological Entomology, 13, 29-42.

Gibson, G., Packer, M. J., Steullet, P., \& Brady, J. (1991). Orientation of tsetse flies to wind, within and outside host odour plumes in the field. Physiological Entomology, 16, 47-56.

Gibson, G., \& Torr, S. J. (1999). Visual and olfactory responses of haematophagous Diptera to host stimuli. Medical and Veterinary Entomology, 13(1), 2-23. 
Gibson, G., Warren, B., \& Russell, I. J. (2010). Humming in tune: Sex and species recognition by mosquitoes on the wing. Journal of the Association for Research in Otolaryngology, 11(4), 527-540.

Gillies, M. T. (1955). Studies on the house leaving and outside resting of Anopheles gambiae Giles and Anopheles funestus Giles in East Africa I.-The exodus from houses and the house resting population. Bulletin of Entomological Research, 45, 375-387.

Gillies, M. T. (1974). Methods for assessing the density and survival of blood-sucking diptera. Annual Review of Entomology, 19, 345-362.

Gillies, M. T. (1980). The role of carbon dioxide in host-finding by mosquitoes (Diptera: Culicidae): A review. Bulletin of Entomological Research, 70(4), 525-532.

Gillies, M. T., \& Wilkes, T. J. (1970). The range of attraction of single baits for some West African mosquitoes. Bulletin of Entomological Research, 60.

Gillies, M. T., \& Wilkes, T. J. (1974a). Evidence for downwind flights by host-seeking mosquitos. Nature, 252, 388-389.

Gillies, M. T., \& Wilkes, T. J. (1974b). The vertical distribution of mosquitoes flying over open farmland in the Gambia. Transactions of the Royal Society of Tropical Medicine and Hygiene, 68(4), 268-269.

Gillies, M. T., \& Wilkes, T. J. (1981). Trapping Host-Seeking Mosquitos with Electrocuting Grids. Transactions of the Royal Society of Tropical Medicine and Hygiene, 75(4), 600-601.

Gillies, M. T., \& Wilkes, T. J. (1982). Responses of host-seeking Mansonia and Anopheles mosquitoes (Diptera: culicidae) in West Africa to visual features of a target. Journal of Medical Entomology, 19(1), 68-71.

Gleave, K., Cook, D., Taylor, M. J., \& Reimer, L. J. (2016). Filarial infection influences mosquito behaviour and fecundity. Scientific Reports, 6, 36319

Grant, A. J., Wigton, B. E., \& Aghajanian, J. G. (1995). Electrophysiological responses of receptor neurons in mosquito maxillary palp sensilla to carbon dioxide. Journal of Comparative Physiology. A, Neuroethology, Sensory, Neural, and Behavioral Physiology, 177, 389-396.

Grieco, J. P., Achee, N. L., Andre, R. G., \& Roberts, D. R. (2000). A comparison study of house entering and exiting behavior of Anopheles vestitipennis (Diptera: Culicidae) using experimental huts sprayed with ddt or deltamethrin in the Southern District of Toledo, Belize, C. A. Journal of Vector Ecology, 25(1), 62-73.

Grigaltchik, V. S., Webb, C., \& Seebacher, F. (2016). Temperature modulates the effects of predation and competition on mosquito larvae. Ecological Entomology, 41(6), 668-675.

Haddow, A. J. (1942). The mosquito fauna and climate of native huts at Kisumu, Kenya. Bulletin of Entomological Research, 33(2), 91-142.

Haddow, A. J. \& Ssenkubuge, Y. (1973). The mosquito of Bwamba county, Uganda. IX. Further studies on the biting behaviour of an outdoor population of the Anopheles gambiae Giles complex. Bulletin of Entomological Research , 62, 407-414.

Hall-Mendelin, S., Ritchie, S. A., Johansen, C. A., Zborowski, P., Cortis, G., Dandridge, S., Hall, R. A., \& van den Hurk, A. F. (2010). Exploiting mosquito sugar feeding to detect mosquito-borne pathogens. Proceedings of the National Academy of Sciences, 107(25), 11255-11259.

Hamer, G. L., Donovan, D. J., Hood-Nowotny, R., Kaufman, M. G., Goldberg, T. L., \& Walker, E. D. (2012). Evaluation of a stable isotope method to mark naturally-breeding larval mosquitoes for adult dispersal studies. Journal of Medical Entomology, 49(1), 61-70.

Hammond, A., Galizi, R., Kyrou, K., Simoni, A., Siniscalchi, C., Katsanos, D., Gribble, M., Baker, D., Marois, E., Russell, S., Burt, A., Windbichler, N., Crisanti, A., \& Nolan, T. (2016). A CRISPRCas9 gene drive system targeting female reproduction in the malaria mosquito vector Anopheles gambiae. Nature Biotechnology, 34(1), 78-83. 
Harris, A. F., Nimmo, D., McKemey, A. R., Kelly, N., Scaife, S., Donnelly, C. A., Beech, C., Petrie, W. D., \& Alphey, L. (2011). Field performance of engineered male mosquitoes. Nature Biotechnology, 29(11), 1034-1037.

Hawkes, F. M., Dabiré, R. K., Sawadogo, S. P., Torr, S. J., \& Gibson, G. (2017). Exploiting Anopheles responses to thermal, odour and visual stimuli to improve surveillance and control of malaria. Scientific Reports, 7(1), 17283.

Hawkes, F. M., \& Gibson, G. (2016). Seeing is believing: the nocturnal malarial mosquito Anopheles coluzzii responds to visual host-cues when odour indicates a host is nearby. Parasites \& Vectors, 9, 320.

Healy, T. P., Copland, M. J., Cork, A., Przyborowska, A., \& Halket, J. M. (2002). Landing responses of Anopheles gambiae elicited by oxocarboxylic acids. Medical and Veterinary Entomology, 16(2), 126-132.

Healy, T. P., \& Copland, M. J. W. (1995). Activation of Anopheles gambiae mosquitos by carbon dioxide and human breath. Medical and Veterinary Entomology, 9(3), 331-336.

Healy, T. P., \& Copland, M. J. W. (2000). Human sweat and 2-oxopentanoic acid elicit a landing response from Anopheles gambiae. Medical and Veterinary Entomology (14), 195-200.

Hedrick, T. L. (2008). Software techniques for two- and three-dimensional kinematic measurements of biological and biomimetic systems. Bioinspiration \& Biomimetics, 3(3), 034001.

Heinig, R. L., \& Thomas, M. B. (2015). Interactions between a fungal entomopathogen and malaria parasites within a mosquito vector. Malaria Journal, 14(1), 22.

Hemingway, J. (2017). The way forward for vector control. Science, 358(6366), 998-999.

Hemingway, J., Ranson, H., Magill, A., Kolaczinski, J., Fornadel, C., Gimnig, J., Coetzee, M., Simard, F., Roch, D. K., Hinzoumbe, C. K., Pickett, J., Schellenberg, D., Gething, P., Hoppé, M., \& Hamon, N. (2016). Averting a malaria disaster: Will insecticide resistance derail malaria control? The Lancet, 387(10029), 1785-1788.

Hiscox, A., Otieno, B., Kibet, A., Mweresa, C. K., Omusula, P., Geier, M., Rose, A., Mukabana, W. R., \& Takken, W. (2014). Development and optimization of the Suna trap as a tool for mosquito monitoring and control. Malaria Journal, 13(1), 257.

Homan, T., Hiscox, A., Mweresa, C. K., Masiga, D., Mukabana, W. R., Oria, P., Maire, N., Pasquale, A. D., Silkey, M., Alaii, J., Bousema, T., Leeuwis, C., Smith, T. A., \& Takken, W. (2016). The effect of mass mosquito trapping on malaria transmission and disease burden (SolarMal): a stepped-wedge cluster-randomised trial. The Lancet, 388(10050), 1193-1201.

Hougard, J. M., Corbel, V., N'Guessan, R., Darriet, F., Chandre, F., Akogbeto, M., Baldet, T., Guillet, P., Carnevale, P., \& Traore-Lamizana, M. (2003). Efficacy of mosquito nets treated with insecticide mixtures or mosaics against insecticide resistant Anopheles gambiae and Culex quinquefasciatus (Diptera: Culicidae) in Cote d'Ivoire. Bulletin of Entomological Research, 93(6), 491-498.

Hounslow, K. (2014). OpenCV Tutorial: Real-time object tracking without colour, https://www.youtube. com/watch?v=X6rPdRZzgjg.

Howlett, F. M. (1910). The influence of temperature upon the biting of mosquitoes. Parasitology, 3 , 479-484.

Huijben, S., \& Paaijmans, K. P. (2017). Putting evolution in elimination: Winning our ongoing battle with evolving malaria mosquitoes and parasites. Evolutionary Applications, 00, 1-16.

Hurd, H. (2003). Manipulation of medically important insect vectors by their parasites. Annual Review of Entomology, 48(1), 141-161.

Imperato, P. J. (2016). The convergence of a virus, mosquitoes, and human travel in globalizing the zika epidemic. Journal of Community Health, 41(3), 674-679. 
Inouye, D. W. (2010). Mosquitoes: More likely nectar thieves than pollinators. Nature, 467(7311), 27.

Itoh, T., Shinjo, G., \& Kurihara, T. (1986). Studies on wide mesh netting impregnated with insecticides against Culex mosquitos. Journal of the American Mosquito Control Association, 2(4), 503-506.

Jawara, M., Smallegange, R. C., Jeffries, D., Nwakanma, D. C., Awolola, T. S., Knols, B. G., Takken, W., \& Conway, D. J. (2009). Optimizing odor-baited trap methods for collecting mosquitoes during the malaria season in The Gambia. PLoS One, 4(12), e8167.

Johnson, B. J., \& Ritchie, S. A. (2016). The Siren's song: Exploitation of female flight tones to passively capture male Aedes aegypti (Diptera: Culicidae). Journal of Medical Entomology, 53(1), 245-248.

Kartzinel, M. A., Alto, B. W., Deblasio, M. W., \& Burkett-Cadena, N. D. (2016). Testing of visual and chemical attractants in correlation with the development and field evaluation of an autodissemination station for the suppression of Aedes aegypti and Aedes albopictus in Florida. Journal of the American Mosquito Control Association, 32(3), 194-202.

Keating, J. A., Bhattacharya, D., Rund, S. S. C., Hoover, S., Dasgupta, R., Lee, S. J., Duffield, G. E., \& Striker, R. (2013). Mosquito protein kinase g phosphorylates flavivirus ns5 and alters flight behavior in Aedes aegypti and Anopheles gambiae. Vector-Borne and Zoonotic Diseases, 13(8), 590-600.

Kellogg, F. E., \& Wright, R. H. (1962). The guidance of flying insects. V. Mosquito Attraction. The Canadian Entomologist, 94, 1009-1016.

Kennedy, J. S. (1942). On water-finding and oviposition by captive mosquitoes. Bulletin of Entomological Research, 32(4), 279-301.

Khan, Z., Midega, C., Pittchar, J., Pickett, J., \& Bruce, T. (2011). Push-pull technology: a conservation agriculture approach for integrated management of insect pests, weeds and soil health in Africa. International Journal of Agricultural Sustainability, 9(1), 162-170.

Killeen, G. F. (2014). Characterizing, controlling and eliminating residual malaria transmission. Malaria Journal, 13, 330.

Killeen, G. F., Chitnis, N., Moore, S. J., \& Okumu, F. O. (2011). Target product profile choices for intradomiciliary malaria vector control pesticide products: repel or kill? Malaria Journal, 10, 207.

Killeen, G. F., Govella, N. J., Lwetoijera, D. W., \& Okumu, F. O. (2016). Most outdoor malaria transmission by behaviourally-resistant Anopheles arabiensis is mediated by mosquitoes that have previously been inside houses. Malaria Journal, 15, 225.

Killeen, G. F., Kihonda, J., Lyimo, E., Oketch, F. R., Kotas, M. E., Mathenge, E., Schellenberg, J. A., Lengeler, C., Smith, T. A., \& Drakeley, C. J. (2006). Quantifying behavioural interactions between humans and mosquitoes: Evaluating the protective efficacy of insecticidal nets against malaria transmission in rural Tanzania. BMC Infectious Diseases, 6, 161.

Killeen, G. F., \& Moore, S. J. (2012). Target product profiles for protecting against outdoor malaria transmission. Malaria Journal, 11, 17.

Killeen, G. F., \& Smith, T. A. (2007). Exploring the contributions of bed nets, cattle, insecticides, and excitorepellency to malaria control: a deterministic model of mosquito host-seeking behaviour and mortality. Transactions of the Royal Society of Tropical Medicine and Hygiene, 101(9), 867-880.

Kilpatrick, A. M. (2011). Globalization, land use, and the invasion of West Nile virus. Science, 334(6054), 323-327.

Kim, J., Jung, M., Kim, H. G., \& Lee, D. H. (2016). Potential of harmonic radar system for use on five economically important insects: Radar tag attachment on insects and its impact on flight capacity. Journal of Asia-Pacific Entomology, 19(2), 371-375.

Kirby, M. J. (2013). House screening. In M. M. Cameron \& L. M. Lorenz (Eds.), Biological and Environmental Control of Disease Vectors (pp. 117-143): CABI.

Kloth, K. J., ten Broeke, C. J. M., Thoen, M. P. M., Hanhart-van den Brink, M., Wiegers, G. L., Krips, 


\section{References}

O. E., Noldus, L. P. J. J., Dicke, M., \& Jongsma, M. A. (2015). High-throughput phenotyping of plant resistance to aphids by automated video tracking. Plant Methods, 11, 4.

Klowden, M. J. (1986). Effects of sugar deprivation on the host-seeking behaviour of gravid Aedes aegypti mosquitoes. Journal of Insect Physiology, 32, 479-483.

Klun, J. A., Kramer, M., \& Debboun, M. (2013). Four simple stimuli that induce host-seeking and blood-feeding behaviors in two mosquito species, with a clue to DEET's mode of action. Journal of Vector Ecology, 38(1), 143-153.

Koehl, M. A. R. (2006). The fluid mechanics of arthropod sniffing in turbulent odor plumes. Chemical Senses, 31(2), 93-105.

Koella, J. C., Sorensen, F. L., \& Anderson, R. A. (1998). The malaria parasite, Plasmodium falciparum, increases the frequency of multiple feeding of its mosquito vector, Anopheles gambiae. Proceedings of the Royal Society B: Biological Sciences, 265, 763-768.

Koenraadt, C. J. M., Majambere, S., Hemerik, L., \& Takken, W. (2004). The effects of food and space on the occurrence of cannibalism and predation among larvae of Anopheles gambiae sl. Entomologia Experimentalis et Applicata, 112(2), 125-134.

Koenraadt, C. J. M., \& Takken, W. (2018). Integrated approach to malaria control. Science, 359(6375), 528-529.

Koffi, A. A., Ahoua Alou, L. P., Djenontin, A., Kabran, J.-P. K., Dosso, Y., Kone, A., Moiroux, N., \& Pennetier, C. (2015). Efficacy of Olyset( $($ ) $)$ Duo, a permethrin and pyriproxyfen mixture net against wild pyrethroid-resistant Anopheles gambiae s.s. from Côte d'Ivoire: an experimental hut trial. Parasite, 22, 28.

Kongmee, M., Boonyuan, W., Achee, N. L., Prabaripai, A., Lerdthusnee, K., \& Chareonviriyaphap, T. (2012). Irritant and repellent responses of Anopheles harrisoni and Anopheles minimus upon exposure to bifenthrin or deltamethrin using an excito-repellency system and a live host. Journal of the American Mosquito Control Association, 28(1), 20-29.

Koudou, B. G., Koffi, A. A., Malone, D., \& Hemingway, J. (2011). Efficacy of PermaNet $® 2.0$ and PermaNet $® 3.0$ against insecticide-resistant Anopheles gambiae in experimental huts in Côte d'Ivoire. Malaria Journal, 10.

Krcmar, S., Hribar, L. J., \& Kopi, M. (2005). Response of Tabanidae (Diptera) to natural and synthetic olfactory attractants. Journal of Vector Ecology, 30(1), 133-136.

Kröner, C., Towers, C. E., Angarita-Jaimes, N., Parker, J. E., McCall, P., \& Towers, D. P. (2016). 3D tracking of mosquitoes: A field compatible technique to understand malaria vector behaviour. Paper presented at Imaging and Applied Optics 2016, Heidelberg.

Kurihara, K., Hoshino, S., Yamane, K., \& Nakamura, Y. (2002). Optical motion capture system with pan-tilt camera tracking and real time data processing. Paper presented at the International Conference on Robotics and Automation (Cat. No.02CH37292).

Laarman, J. J. (1958). The host-seeking behaviour of anopheline mosquitoes. Tropical and Geographical Medicine, 10(4), 293-305.

Lacey, E. S., \& Cardé, R. T. (2011). Activation, orientation and landing of female Culex quinquefasciatus in response to carbon dioxide and odour from human feet: 3-D flight analysis in a wind tunnel. Medical and Veterinary Entomology, 25(1), 94-103.

Lacey, E. S., \& Cardé, R. T. (2012). Location of and landing on a source of human body odour by female Culex quinquefasciatus in still and moving air. Physiological Entomology, 37(2), 153159.

Lacey, E. S., Ray, A., \& Cardé, R. T. (2014). Close encounters: contributions of carbon dioxide and human skin odour to finding and landing on a host in Aedes aegypti. Physiological Entomology, $39(1), 60-81$. 
Lacroix, R., Mukabana, W. R., Gouagna, L. C., \& Koella, J. C. (2005). Malaria infection increases attractiveness of humans to mosquitoes. PLoS Biology, 3(9), e298.

Lee, J. H., Rowley, W. A., \& Platt, K. B. (2000). Longevity and spontaneous flight activity of Culex tarsalis (Diptera: Culicidae) infected with Western equine encephalomyelitis virus. Journal of Medical Entomology, 37(1), 187-193.

Lefèvre, T., Gouagna, L. C., Dabire, K. R., Elguero, E., Fontenille, D., Renaud, F., Costantini, C., \& Thomas, F. (2009). Beyond nature and nurture: Phenotypic plasticity in blood-feeding behavior of Anopheles gambiae s.s. when humans are not readily accessible. American Journal of Tropical Medicine and Hygiene, 81(6), 1023-1029.

Lefèvre, T., \& Thomas, F. (2008). Behind the scene, something else is pulling the strings: emphasizing parasitic manipulation in vector-borne diseases. Infection, Genetics and Evolution, 8(4), 504-519.

Lehmann, T., \& Diabate, A. (2008). The molecular forms of Anopheles gambiae: A phenotypic perspective. Infection, Genetics and Evolution, 8(5), 737-746.

Lengeler, C. (2004a). Insecticide-treated bed nets and curtains for preventing malaria. Cochrane Database of Systematic Reviews, 2.

Lengeler, C. (2004b). Insecticide-treated nets for malaria control: real gains. Bulletin of the World Health Organization, 82(2), 84.

Li, J., Deng, T., Li, H., Chen, L., \& Mo, J. (2009). Effects of water color and chemical compounds on the oviposition behavior of gravid Culex pipiens pallens females under Laboratory conditions. Journal of Agricultural and Urban Entomology, 26(1), 23-30.

Lim, S. S., Fullman, N., Stokes, A., Ravishankar, N., Masiye, F., Murray, C. J. L., \& Gakidou, E. (2011). Net Benefits: A Multicountry Analysis of Observational Data Examining Associations between Insecticide-Treated Mosquito Nets and Health Outcomes. PLoS Medicine, 8(9), e1001091.

Lindsay, S. W., Adiamah, J. H., Miller, J. E., \& Armstrong, J. R. (1991). Pyrethroid-treated bednet effects on mosquitoes of the Anopheles gambiae complex in The Gambia. Medical and Veterinary Entomology, 5(4), 477-483.

Lindsay, S. W., Emerson, P. M., \& Charlwood, J. D. (2002). Reducing malaria by mosquito-proofing houses. Trends in Parasitology, 18(11), 510-4.

Lindsay, S. W., \& Snow, R. W. (1988). The trouble with eaves: house entry by vectors of malaria. Transactions of the Royal Society of Tropical Medicine and Hygiene, 82(4), 645-646.

Lindsay, S. W., Snow, R. W., Broomfield, G. L., Janneh, M. S., Wirtz, R. A., \& Greenwood, B. M. (1989). Impact of permethrin-treated bednets on malaria transmission by the Anopheles gambiae complex in The Gambia. Medical and Veterinary Entomology, 3(3), 263-271.

Lines, J. D., Myamba, J., \& Curtis, C. F. (1987). Experimental hut trials of permethrin-impregnated mosquito nets and eave curtains against malaria vectors in Tanzania. Medical and Veterinary Entomology, 1, 37-51.

Liu, C., Pitts, R. J., Bohbot, J. D., Jones, P. L., Wang, G., \& Zwiebel, L. J. (2010). Distinct olfactory signaling mechanisms in the malaria vector mosquito Anopheles gambiae. PLoS Biology, 8(8), 27-28.

Liu, C., \& Zwiebel, L. J. (2013). Molecular characterization of larval peripheral thermosensory responses of the malaria vector mosquito Anopheles gambiae. PLoS One, 8(8), e72595.

Loon, J. J. A., Smallegange, R. C., Bukovinszkiné-Kiss, G., Jacobs, F., Rijk, M., \& Mukabana, W. R. (2015). Mosquito attraction: crucial role of carbon dioxide in formulation of a five-component blend of human-derived volatiles. Journal of Chemical Ecology, 41(6), 567-573.

Lorenz, L. M., Keane, A., Moore, J. D., Munk, C. J., Seeholzer, L., Mseka, A., Simfukwe, E., Ligamba, J., Turner, E. L., Biswaro, L. R., Okumu, F. O., Killeen, G. F., Mukabana, W. R., \& Moore, S. J. (2013). Taxis assays measure directional movement of mosquitoes to olfactory cues. Parasites \& Vectors, 6(1), 131. 


\section{References}

Lu, T., Qiu, Y. T., Wang, G., Kwon, J. Y., Rutzler, M., Kwon, H. W., Pitts, R. J., van Loon, J. J., Takken, W., Carlson, J. R., \& Zwiebel, L. J. (2007). Odor coding in the maxillary palp of the malaria vector mosquito Anopheles gambiae. Current Biology, 17(18), 1533-1544.

Lyimo, I. N., \& Ferguson, H. M. (2009). Ecological and evolutionary determinants of host species choice in mosquito vectors. Trends in Parasitology, 25(4), 189-196.

Lynch, P. A., \& Boots, M. (2016). Using evolution to generate sustainable malaria control with spatial repellents. eLife, 5, e15416.

Lynd, A., \& McCall, P. J. (2013). Clustering of host-seeking activity of Anopheles gambiae mosquitoes at the top surface of a human-baited bed net. Malaria Journal, 12(1), 267.

Mafra-Neto, A., \& Cardé, R. T. (1994). Fine-scale structure of pheromone plumes modulates upwind orientation of flying moths. Nature, 369, 142-144.

Mafra-Neto, A., \& Cardé, R. T. (1995a). Influence of plume structure and pheromone concentration on upwind flight of Cadra cautella males. Physiological Entomology, 20, 117-133.

Mafra-Neto, A., \& Cardé, R. T. (1995b). Effect of the fine-scale structure of pheromone plumes: pulse frequency modulates activation and upwind flight of almond moth males. Physiological Entomology, 20, 229-242.Magesa, S. M., Wilkes, T. J., Mnzava, A. E., Njunwa, K. J., Myamba, J., \& Kivuyo, M. D. (1991). Trial of pyrethroid impregnated bednets in an area of Tanzania holoendemic for malaria. Part 2. Effects on the malaria vector population. Acta Tropica, 49, 97-108.

Maïga, H., Niang, A., Sawadogo, S. P., Dabiré, R. K., Lees, R. S., Gilles, J. R. L., Tripet, F., \& Diabaté, A. (2014). Role of nutritional reserves and body size in Anopheles gambiae males mating success. Acta Tropica, 132(1), S102-S107.

Majeed, S., Hill, S. R., Dekker, T., \& Ignell, R. (2017). Detection and perception of generic host volatiles by mosquitoes: Responses to $\mathrm{CO}_{2}$ constrains host-seeking behaviour. Royal Society Open Science, 4(5), 170189.

Malima, R. C., Oxborough, R. M., Tungu, P. K., Maxwell, C., Lyimo, I., Mwingira, V., Mosha, F. W., Matowo, J., Magesa, S. M., \& Rowland, M. W. (2009). Behavioural and insecticidal effects of organophosphate-, carbamate- and pyrethroid-treated mosquito nets against African malaria vectors. Medical and Veterinary Entomology, 23(4), 317-325.

Manoukis, N. C., Diabate, A., Abdoulaye, A., Diallo, M., Dao, A., Yaro, A. S., Ribeiro, J. M., \& Lehmann, T. (2009). Structure and dynamics of male swarms of Anopheles gambiae. Journal of Medical Entomology, 46(2), 227-235.

Massue, D., Kisinza, W., Malongo, B., Mgaya, C., Bradley, J., Moore, J., Tenu, F., \& Moore, S. (2016). Comparative performance of three experimental hut designs for measuring malaria vector responses to insecticides in Tanzania. Malaria Journal, 15(1), 165.

Mathenge, E. M., Gimnig, J. E., Kolczak, M., Ombok, M., Irungu, L. W., \& Hawley, W. A. (2001). Effect of permethrin-impregnated nets on exiting behavior, blood feeding success, and time of feeding of malaria mosquitoes (Diptera: Culicidae) in western Kenya. Journal of Medical Entomology, 38(4), 531-536.

Matowo, N. S., Koekemoer, L. L., Moore, S. J., Mmbando, A. S., Mapua, S. A., Coetzee, M., \& Okumu, F. O. (2016). Combining synthetic human odours and low-cost electrocuting grids to attract and kill outdoor-biting mosquitoes: Field and semi-field evaluation of an improved mosquito landing box. PLoS One, 11(1), e0145653.

Maxwell, C.A., Wakibara, J., Tho, S. \& Curtis, C.F. (1998). Malaria-infective biting at different hours of the night. Medical and Veterinary Entomology, 12, 325-327.

Mboera, L. E. G., Knols, B. G. J., Braks, M. A. H., \& Takken, W. (2000b). Comparison of carbon dioxide-baited trapping systems for sampling outdoor mosquito populations in Tanzania. Medical and Veterinary Entomology, 14, 257-263. 
Mboera, L. E. G., \& Takken, W. (1997). Carbon dioxide chemotropism in mosquitoes (Diptera: Culicidae) and its potential in vector surveillance and management programmes. Review of Applied Entomologie series B, Medical and Veterinary Entomology, 85, 355-368.

Mboera, L. E. G., \& Takken, W. (1999). Odour-mediated host preference of Culex quinquefasciatus in Tanzania. Entomologia Experimentalis et Applicata, 92, 83-88.

Mboera, L. E. G., Takken, W., Mdira, K. Y., \& Pickett, J. A. (2000a). Sampling gravid Culex quinquefasciatus (Diptera: Culicidae) in Tanzania with traps baited with synthetic oviposition pheromone and grass infusions. Journal of Medical Entomology, 37(1), 172-176.

Mboera, L. E. G., Takken, W., \& Sambu, E. Z. (2000c). The response of Culex quinquefasciatus (Diptera: Culicidae) to traps baited with carbon dioxide, 1-octen-3-ol, acetone, butyric acid and human foot odour in Tanzania. Bulletin of Entomological Research, 90, 155-159.

Mburu, M. M., Mweresa, C. K., Omusula, P., Hiscox, A., Takken, W., \& Mukabana, W. R. (2017). 2-Butanone as a carbon dioxide mimic in attractant blends for the Afrotropical malaria mosquitoes Anopheles gambiae and Anopheles funestus. Malaria Journal, 16(1), 351.

McCann, R. S., van den Berg, H., Diggle, P. J., van Vugt, M., Terlouw, D. J., Phiri, K. S., Di Pasquale, A., Maire, N., Gowelo, S., Mburu, M. M., Kabaghe, A. N., Mzilahowa, T., Chipeta, M. G., \& Takken, W. (2017). Assessment of the effect of larval source management and house improvement on malaria transmission when added to standard malaria control strategies in southern Malawi: study protocol for a cluster-randomised controlled trial. BMC Infectious Diseases, 17(1), 639.

McMeniman, C. J., Corfas, R. A., Matthews, B. J., Ritchie, S. A., \& Vosshall, L. B. (2014). Multimodal integration of carbon dioxide and other sensory cues drives mosquito attraction to humans. Cell, 156(5), 1060-1071.

Medlock, J. M., \& Leach, S. A. (2015). Effect of climate change on vector-borne disease risk in the UK. The Lancet Infectious Diseases, 15(6), 721-730.

Menger, D. J., Omusula, P., Holdinga, M., Homan, T., Carreira, A. S., Vandendaele, P., Derycke, J. L., Mweresa, C. K., Mukabana, W. R., van Loon, J. J. A., \& Takken, W. (2015). Field evaluation of a push-pull system to reduce malaria transmission. PLoS One, 10(4).

Menger, D. J., Omusula, P., Wouters, K., Oketch, C., Carreira, A. S., \& Durka, M. (2016). Eave screening and push-pull tactics to reduce house entry by vectors of malaria. American Journal of Tropical Medicine and Hygiene, 94(4), 868-878.

Menger, D. J., Otieno, B., de Rijk, M., Mukabana, W. R., van Loon, J. J., \& Takken, W. (2014b). A push-pull system to reduce house entry of malaria mosquitoes. Malaria Journal, 13, 119.

Menger, D. J., van Loon, J. J. A., \& Takken, W. (2014a). Assessing the efficacy of candidate mosquito repellents against the background of an attractive source that mimics a human host. Medical and Veterinary Entomology, 28(4), 407-413.

Michaelakis, A., Mihou, A. P., Koliopoulos, G., \& Couladouros, E. A. (2007). Attract-and-kill strategy. Laboratory studies on hatched larvae of Culex pipiens. Pest Management Science, 63(10), 954-959.

Microsoft. (2015). ProjectPremonition, Robotic mosquito traps that identify and capture interesting mosquitoes in milliseconds. https://www.microsoft.com/en-us/research/project/project-premonition/\#.

Miller, J. E., \& Gibson, G. (1994). Behavioral response of host-seeking mosquitos (Diptera, Culicidae) to insecticide-impregnated bed netting - a new approach to insecticide bioassays. Journal of Medical Entomology, 31(1), 114-122.

Miller, J. E., Lindsay, S. W., \& Armstrong, J. R. (1991). Experimental hut trials of bednets impregnated with synthetic pyrethroid or organophosphate insecticide for mosquito control in The Gambia. Medical and Veterinary Entomology, 5(4), 465-476.

Mnyone, L. L., Kirby, M., Lwetoijera, D., Mpingwa, M., Simfukwe, E., Knols, B., Takken, W., \& Russell, T. (2010). Tools for delivering entomopathogenic fungi to malaria mosquitoes: effects of delivery surfaces on fungal efficacy and persistence. Malaria Journal, 9, 246. 


\section{References}

Mnyone, L. L., Lyimo, I. N., Lwetoijera, D. W., Mpingwa, M. W., Nchimbi, N., \& Hancock, P. A. (2012). Exploiting the behaviour of wild malaria vectors to achieve high infection with fungal biocontrol agents. Malaria Journal, 11, 87.

Moiroux, N., Gomez, M. B., Pennetier, C., Elanga, E., Djenontin, A., Chandre, F., Djegbe, I., Guis, H., \& Corbel, V. (2012). Changes in Anopheles funestus biting behavior following universal coverage of long-lasting insecticidal nets in Benin. Journal of Infectious Diseases, 206(10), 1622-1629.

Montell, C., Zwiebel, L. J., \& Alexander, S. R. (2016). Mosquito Sensory Systems Advances in Insect Physiology (Vol. Volume 51, pp. 293-328): Academic Press.

Moore, A., Miller, J. R., Tabashnik, B. E., \& Gage, S. H. (1986). Automated identification of flying insects by analysis of wingbeat frequencies. Journal of Economic Entomology, 79(6), 1703-1706.

Moores, G., Bingham, G., \& Gunning, R. (2005). Use of 'temporal synergism' to overcome insecticide resistance. Outlooks on Pest Management, 16(1), 7-9.

Moreira, L. A., Saig, E., Turley, A. P., Ribeiro, J. M. C., O’Neill, S. L., \& McGraw, E. A. (2009). Human probing behavior of Aedes aegypti when infected with a life-shortening strain of Wolbachia. PLoS Neglected Tropical Diseases, 3(12), e568.

Mosha, F. W., Lyimo, I. N., Oxborough, R. M., Matowo, J., Malima, R., Feston, E., Mndeme, R., Tenu, F., Kulkarni, M., Maxwell, C. A., Magesa, S. M., \& Rowland, M. W. (2008). Comparative efficacies of permethrin-, deltamethrin- and alpha-cypermethrin-treated nets, against Anopheles arabiensis and Culex quinquefasciatus in northern Tanzania. Annals of tropical medicine and parasitology, 102(4), 367-376.

Mouden, S., Sarmiento, K. F., Klinkhamer, P. G. L., \& Leiss, K. A. (2017). Integrated pest management in western flower thrips: past, present and future. Pest Management Science, 73(5), 813-822.

Muijres, F. T., Chang, S. W., van Veen, W. G., Spitzen, J., Biemans, B. T., Koehl, M. A. R., \& Dudley, R. (2017). Escaping blood-fed malaria mosquitoes minimize tactile detection without compromising on take-off speed. Journal of Experimental Biology, 220(20), 3751-3762.

Muir, L. E., Kay, B. H., \& Thorne, M. J. (1992). Aedes aegypti (Diptera: Culicidae) vision: response to stimuli from the optical environment. Journal of Medical Entomology, 29(3), 445-450.

Mukabana, W. R., Mweresa, C. K., Otieno, B., Omusula, P., Smallegange, R. C., Loon, J. J. A. v., \& Takken, W. (2012). A novel synthetic odorant blend for trapping of malaria and other African mosquito species. Journal of Chemical Ecology, 38(3), 235-244.

Mukabana, W. R., Olanga, E. A., \& Knols, B. G. J. (2010). Host-seeking behaviour of Afrotropical anophelines: field and semi-field studies. In W. Takken \& B. G. J. Knols (Eds.), Olfaction in vector-host interactions. Wageningen: Wageningen Academic Publishers.

Mukundarajan, H., Hol, F. J. H., Castillo, E. A., Newby, C., \& Prakash, M. (2017). Using mobile phones as acoustic sensors for high-throughput mosquito surveillance. eLife, 6, e27854.

Müller, G. C., Beier, J. C., Traore, S. F., Toure, M. B., Traore, M. M., Bah, S., Doumbia, S., \& Schlein, Y. (2010). Successful field trial of attractive toxic sugar bait (ATSB) plant-spraying methods against malaria vectors in the Anopheles gambiae complex in Mali, West Africa. Malaria Journal, 9(1), 210.

Murlis, J., Elkinton, J. S., \& Cardé, R. T. (1992). Odor plumes and how insects use them. Annual Review of Entomology, 37, 505-532.

Murray, C. J. L., Rosenfeld, L. C., Lim, S. S., Andrews, K. G., Foreman, K. J., Haring, D., Fullman, N., Naghavi, M., Lozano, R., \& Lopez, A. D. (2012). Global malaria mortality between 1980 and 2010: a systematic analysis. The Lancet, 379(9814), 413-431.

N'Guessan, R., Asidi, A., Boko, P., Odjo, A., Akogbeto, M., Pigeon, O., \& Rowland, M. (2010). An experimental hut evaluation of PermaNet $₫ 3.0$, a deltamethrin-piperonyl butoxide combination 
net, against pyrethroid-resistant Anopheles gambiae and Culex quinquefasciatus mosquitoes in southern Benin. Transactions of the Royal Society of Tropical Medicine and Hygiene, 104(12), 758-765.

N'Guessan, R., Corbel, V., Akogbeto, M., \& Rowland, M. (2007). Reduced efficacy of insecticidetreated nets and indoor residual spraying for malaria control in pyrethroid resistance area, Benin. Emerging Infectious Diseases, 13(2), 199-206.

Nayar, J. K., \& van Handel, W. (1971). The fuel for sustained mosquito flight. Journal of Insect Physiology, 17, 471-481.

Newman, C. M., Anderson, T. K., \& Goldberg, T. L. (2016). Decreased flight activity in Culex pipiens (Diptera: Culicidae) naturally infected with Culex flavivirus. Journal of Medical Entomology, 53(1), 233-236.

Njiru, B. N., Mukabana, W. R., Takken, W., \& Knols, B. G. (2006). Trapping of the malaria vector Anopheles gambiae with odour-baited MM-X traps in semi-field conditions in western Kenya. Malaria Journal, 5(1), 39.

Nyasembe, V. O., Teal, P. E. A., Sawa, P., Tumlinson, J. H., Borgemeister, C., \& Torto, B. (2014). Plasmodium falciparum infection increases Anopheles gambiae attraction to nectar sources and sugar uptake. Current Biology, 24(2), 217-221.

Offenhauser Jr, W. H., \& Kahn, M. C. (1949). The sounds of disease-carrying mosquitoes. The Journal of the Acoustical Society of America, 21(3), 259-263.

Ogoma, S. B., Lweitoijera, D. W., Ngonyani, H., Furer, B., Russell, T. L., \& Mukabana, W. R. (2010). Screening mosquito house entry points as a potential method for integrated control of endophagic filariasis, arbovirus and malaria vectors. PLoS Neglected Tropical Diseases, 4(8), e773.

Okal, M. N., Lindh, J. M., Torr, S. J., Masinde, E., Orindi, B., Lindsay, S. W., \& Fillinger, U. (2015). Analysing the oviposition behaviour of malaria mosquitoes: Design considerations for improving two-choice egg count experiments. Malaria Journal, 14, 250.

Okumu, F. O., Killeen, G. F., Ogoma, S., Biswaro, L., Smallegange, R. C., Mbeyela, E., Titus, E., Munk, C., Ngonyani, H., Takken, W., Mshinda, H., Mukabana, W. R., \& Moore, S. J. (2010). Development and field evaluation of a synthetic mosquito lure that Is more attractive than humans. PLoS One, 5(1), e8951.

Okumu, F. O., Moore, J., Mbeyela, E., Sherlock, M., Sangusangu, R., Ligamba, G., Russell, T., \& Moore, S. J. (2012). A modified experimental hut design for studying responses of diseasetransmitting mosquitoes to indoor interventions: The Ifakara experimental huts. PLoS One, 7(2), e30967.

Olanga, E. A., Okal, M. N., Mbadi, P. A., Kokwaro, E. D., \& Mukabana, W. R. (2010). Attraction of Anopheles gambiae to odour baits augmented with heat and moisture. Malaria Journal, 9, 6 .

Oliva, C. F., Damiens, D., \& Benedict, M. Q. (2014). Male reproductive biology of Aedes mosquitoes. Acta Tropica, 132(1), S12-S19.

Otienoburu, P. E., Nikbakhtzadeh, M. R., \& Foster, W. A. (2016). Orientation of Anopheles gambiae (Diptera: Culicidae) to plant-host volatiles in a novel diffusion-cage olfactometer. Journal of Medical Entomology, 53(1), 237-240.

Ouyang, T.-H., Yang, E.-C., Jiang, J.-A., \& Lin, T.-T. (2015). Mosquito vector monitoring system based on optical wingbeat classification. Computers and Electronics in Agriculture, 118, 47-55.

Oxborough, R. M., Kitau, J., Mosha, F. W., \& Rowland, M. W. (2015). Modified veranda-trap hut for improved evaluation of vector control interventions. Medical and Veterinary Entomology, 29(4), 371-379.

Parker, J. E. A., Angarita-Jaimes, N., Abe, M., Towers, C. E., Towers, D., \& McCall, P. (2015). Infrared video tracking of Anopheles gambiae at insecticide-treated bed nets reveals rapid decisive impact after brief localised net contact. Scientific Reports, 5, 13392. 
Parker, J. E. A., Angarita Jaimes, N. C., Gleave, K., Mashauri, F., Abe, M., Martine, J., Towers, C. E., Towers, D., \& McCall, P. J. (2017). Host-seeking activity of a Tanzanian population of Anopheles arabiensis at an insecticide treated bed net. Malaria Journal, 16(1), 270.

Pates, H. V., Takken, W., Stuke, K., \& Curtis, C. F. (2001). Differential behaviour of Anopheles gambiae sensu stricto (Diptera: Culicidae) to human and cow odours in the laboratory. Bulletin of Entomological Research, 91, 289-296.

Patil, P. B., Niranjan Reddy, B. P., Gorman, K., Seshu Reddy, K. V., Barwale, S. R., Zehr, U. B., Nimmo, D., Naish, N., \& Alphey, L. (2015). Mating competitiveness and life-table comparisons between transgenic and Indian wild-type Aedes aegypti L. Pest Management Science, 71(7), 957-965.

Peach, D. A. H., \& Gries, G. (2016). Nectar thieves or invited pollinators? A case study of tansy flowers and common house mosquitoes. Arthropod-Plant Interactions, 1-10.

Perez-Escudero, A., Vicente-Page, J., Hinz, R. C., Arganda, S., \& de Polavieja, G. G. (2014). idTracker: tracking individuals in a group by automatic identification of unmarked animals. Nature Methods, 11(7), 743-748.

Peterson, D. G., \& Brown, A. W. A. (1951). Studies of the responses of the female Aedes mosquito IIIthe response of Aedes aegypti (L.) to a warm body and its radiation. Bulletin of Entomological Research, 42, 535-541.

Philippe-Janon, J. C. D., van den Hurk, A. F., Francis, D. P., Shivas, M. A., \& Jansen, C. C. (2015). Field comparison of cyclopentanone versus carbon dioxide as an attractant for adult mosquitoes in Southeast Queensland, Australia. Journal of Medical Entomology, 52(3), 483-490.

Phillips-Howard, P. A., ter Kuile, F. O., Nahlen, B. L., Alaii, J. A., Gimnig, J. E., Kolczak, M. S., Terlouw, D. J., Kariuki, S. K., Shi, Y. P., Kachur, S. P., Hightower, A. W., Vulule, J. M., \& Hawley, W. A. (2003). The efficacy of permethrin-treated bed nets on child mortality and morbidity in western Kenya II. Study design and methods. American Journal of Tropical Medicine and Hygiene, 68(4), 10-15.

Poh, A. H., Moghavvemi, M., Leong, C. S., Lau, Y. L., Safdari Ghandari, A., Apau, A., \& Mahamd Adikan, F. R. (2017). Collective behavior quantification on human odor effects against female Aedes aegypti mosquitoes-Open source development. PLoS One, 12(2), e0171555.

Potamitis, I., \& Rigakis, I. (2016). Measuring the fundamental frequency and the harmonic properties of the wingbeat of a large number of mosquitoes in flight using $2 \mathrm{D}$ optoacoustic sensors. Applied Acoustics, 109, 54-60.

Potamitis, I., Rigakis, I., \& Fysarakis, K. (2015). Insect biometrics: Optoacoustic signal processing and its applications to remote monitoring of McPhail type traps. PLoS One, 10(11), e0140474.

Pruszynski, C. (2016). The Bg-counter: A new surveillance trap that remotely measures mosquito density in real-time. Wing Beats, 27, 13.

Qiu, Y. T., Gort, G., Torricelli, R., Takken, W., \& van Loon, J. J. A. (2013). Effects of blood-feeding on olfactory sensitivity of the malaria mosquito Anopheles gambiae: Application of mixed linear models to account for repeated measurements. Journal of Insect Physiology, 59(11), 1111-1118.

Qiu, Y. T., Smallegange, R. C., Ter Braak, C. J. F., Spitzen, J., van Loon, J. J. A., Jawara, M., Milligan, P., Galimard, A. M., Van Beek, T. A., Knols, B. G. J., \& Takken, W. (2007). Attractiveness of MM-X traps baited with human or synthetic odor to mosquitoes (Diptera : Culicidae) in the Gambia. Journal of Medical Entomology, 44(6), 970-983.

Qiu, Y. T., Smallegange, R. C., van Loon, J. J. A., \& Takken, W. (2011). Behavioural responses of Anopheles gambiae sensu stricto to components of human breath, sweat and urine depend on mixture composition and concentration. Medical and Veterinary Entomology, 25(3), 247255. 
Qiu, Y. T., Smallegange, R. C., van Loon, J. J. A., Ter Braak, C. J. F., \& Takken, W. (2006). Interindividual variation in the attractiveness of human odours to the malaria mosquito Anopheles gambiae s.s. Medical and Veterinary Entomology, 20(3), 280-7.

Qualls, W. A., Müller, G. C., Traore, S. F., Traore, M. M., Arheart, K. L., Doumbia, S., Schlein, Y., Kravchenko, V. D., Xue, R. D., \& Beier, J. C. (2015). Indoor use of attractive toxic sugar bait (ATSB) to effectively control malaria vectors in Mali, West Africa. Malaria Journal, 14(1), 301. Quiroz-Martinez, H., Garza-Rodriguez, M. I., Trujillo-Gonzalez, M. I., Zepeda-Cavazos, I. G., SillerAguillon, I., Martinez-Perales, J. F., \& Rodriguez-Castro, V. A. (2012). Selection of oviposition sites by female Aedes aegypti exposed to two larvicides. Journal of the American Mosquito Control Association, 28(1), 47-49.

Raman, D. R., Gerhardt, R. R., \& Wilkerson, J. B. (2007). Detecting insect flight sounds in the field: Implications for acoustical counting of mosquitoes. Transactions of the ASABE, 50(4), 1481-1485.

Ranson, H., \& Lissenden, N. (2016). Insecticide resistance in African Anopheles mosquitoes: a worsening situation that needs urgent action to maintain malaria control. Trends in Parasitology, 32(3), 187-196.

Ranson, H., N'Guessan, R., Lines, J., Moiroux, N., Nkuni, Z., \& Corbel, V. (2011). Pyrethroid resistance in African anopheline mosquitoes: what are the implications for malaria control? Trends in Parasitology, 27(2), 91-98.

Ray, A. (2015). Reception of odors and repellents in mosquitoes. Current Opinion in Neurobiology, 34, 158-164.

Rezza, G. (2014). Dengue and Chikungunya: Long-distance spread and outbreaks in naïve areas. Pathogens and Global Health, 108(8), 349-355.

Roberts, D. R., Alecrim, W. D., Hshieh, P., Grieco, J. P., Bangs, M., Andre, R. G., \& Chareonviriphap, T. (2000). A probability model of vector behavior: effects of DDT repellency, irritancy, and toxicity in malaria control. Journal of Vector Ecology, 25(1), 48-61.

Rudolfs, W. (1922). Chemotropism of mosquitoes. Bulletin New Jersey Agricultural Experiment Station, 367, 4-23.

Ruigt, G. S. F. (1985). Pyrethroids. In G. A. Kerkut \& L. I. Gilbert (Eds.), Comprehensive insect physiology biochemistry and pharmacology (Vol. 12, pp. 183-262). Oxford: Pergamon.

Rund, S. S., Lee, S. J., Bush, B. R., \& Duffield, G. E. (2012). Strain- and sex-specific differences in daily flight activity and the circadian clock of Anopheles gambiae mosquitoes. Journal of Insect Physiology, 58(12), 1609-1619.

Russell, T. L., Beebe, N. W., Cooper, R. D., Lobo, N. F., \& Burkot, T. R. (2013). Successful malaria elimination strategies require interventions that target changing vector behaviours. Malaria Journal, 12, 56.

Rutkowski, A. J., Quinn, R. D., \& Willis, M. A. (2009). Three-dimensional characterization of the wind-borne pheromone tracking behavior of male hawkmoths, Manduca sexta. Journal of Comparative Physiology. A, Neuroethology, Sensory, Neural, and Behavioral Physiology, 195(1), 39-54.

Saiz, J. C., Vázquez-Calvo, Á., Blázquez, A. B., Merino-Ramos, T., Escribano-Romero, E., \& MartínAcebes, M. A. (2016). Zika virus: The latest newcomer. Frontiers in Microbiology, 7, 496.

Sawicki, R. (1962). Insecticidal activity of pyrethrum extract and its four insecticidal constituents against house flies. III.-Knock-down and recovery of flies treated with pyrethrum extract with and without piperonyl butoxide. Journal of the Science of Food and Agriculture, 13(5), 283-292.

Scholte, E. J., Knols, B. G., \& Takken, W. (2006). Infection of the malaria mosquito Anopheles gambiae with the entomopathogenic fungus Metarhizium anisopliae reduces blood feeding and fecundity. Journal of Invertebrate Pathology, 91(1), 43-49.

Scholte, E. J., Ng'habi, K., Kihonda, J., Takken, W., Paaijmans, K., Abdulla, S., Killeen, G. F., \& Knols, 


\section{References}

B. G. (2005). An entomopathogenic fungus for control of adult African malaria mosquitoes. Science, 308(5728), 1641-1642.

Schorkopf, D. L. P., Spanoudis, C. G., Mboera, L. E. G., Mafra-Neto, A., Ignell, R., \& Dekker, T. (2016). Combining attractants and larvicides in biodegradable matrices for sustainable mosquito vector control. PLoS Neglected Tropical Diseases, 10(10), e0005043.

Schreck, C. E., \& Kline, D. L. (1983). Area protection by use of repellent-treated netting against culicoides biting midges. Mosquito News, 43(3), 338-342.

Scott, T. W., \& Takken, W. (2012). Feeding strategies of anthropophilic mosquitoes result in increased risk of pathogen transmission. Trends in Parasitology, 28(3), 114-121.

Siegert, P. Y., Walker, E., \& Miller, J. R. (2009). Differential behavioral responses of Anopheles gambiae (Diptera: Culicidae) modulate mortality caused by pyrethroid-treated bednets. Journal of Economic Entomology, 102(6), 2061-2071.

Simões, P. M. V., Gibson, G., \& Russell, I. J. (2017). Pre-copula acoustic behaviour of males in the malarial mosquitoes Anopheles coluzzii and Anopheles gambiae s.s. does not contribute to reproductive isolation. Journal of Experimental Biology, 220(3), 379-385.

Simões, P. M. V., Ingham, R. A., Gibson, G., \& Russell, I. J. (2016). A role for acoustic distortion in novel rapid frequency modulation behaviour in free-flying male mosquitoes. Journal of Experimental Biology, 219(13), 2039-2047.

Singh, K., Rahman, S. J., \& Joshi, G. C. (1989). Village scale trial of deltamethrin against mosquitoes. Journal of Communicable Diseases, 21(4), 339-353.

Sinka, M. E., Bangs, M., Manguin, S., Coetzee, M., Mbogo, C., Hemingway, J., Patil, A., Temperley, W., Gething, P., Kabaria, C., Okara, R., van Boeckel, T., Godfray, H. C., Harbach, R., \& Hay, S. (2010). The dominant Anopheles vectors of human malaria in Africa, Europe and the Middle East: occurrence data, distribution maps and bionomic precis. Parasites \& Vectors, 3(1), 117.

Sinka, M. E., Bangs, M. J., Manguin, S., Rubio-Palis, Y., Chareonviriyaphap, T., Coetzee, M., Mbogo, C. M., Hemingway, J., Patil, A. P., Temperley, W. H., Gething, P. W., Kabaria, C. W., Burkot, T. R., Harbach, R. E., \& Hay, S. I. (2012). A global map of dominant malaria vectors. Parasites \& Vectors, 5(1), 69.

Smallegange, R. C., Knols, B. G. J., \& Takken, W. (2010a). Effectiveness of synthetic versus natural human volatiles as attractants for Anopheles gambiae (Diptera: Culicidae) sensu stricto. Journal of Medical Entomology, 47(3), 338-344.

Smallegange, R. C., Qu, Y. T., van Loon, J. J., \& Takken, W. (2005). Synergism between ammonia, lactic acid and carboxylic acids as kairomones in the host-seeking behaviour of the malaria mosquito Anopheles gambiae sensu stricto (Diptera: Culicidae). Chemical Senses, 30(2), 145-152.

Smallegange, R. C., Schmied, W. H., van Roey, K. J., Verhulst, N. O., Spitzen, J., Mukabana, W. R., \& Takken, W. (2010b). Sugar-fermenting yeast as an organic source of carbon dioxide to attract the malaria mosquito Anopheles gambiae. Malaria Journal, 9, 292.

Smallegange, R. C., van Gemert, G. J., van de Vegte-Bolmer, M., Gezan, S., Takken, W., Sauerwein, R. W., \& Logan, J. G. (2013). Malaria infected mosquitoes express enhanced attraction to human odor. PLoS One, 8(5), e63602.

Snow, W. F. (1970). The effect of a reduction in expired carbon dioxide on the attractiveness of human subjects to mosquitoes. Bulletin of Entomological Research, 60, 43-48.

Snow, W. F. (1987). Studies of house-entering habits of mosquitoes in The Gambia, West Africa: experiments with prefabricated huts with various wall apertures. Medical and Veterinary Entomology, 1(1), 9-21.

Stange, G. (1997). Effects of changes in atmospheric carbon dioxide on the location of hosts by the moth, Cactoblastis cactorum. Oecologia, 110, 539-545. 
Sperling, S., Cordel, M., Gordon, S., Knols, B., \& Rose, A. (2017). Eave tubes for malaria control in africa: Videographic observations of mosquito behaviour in tanzania with a simple and rugged video surveillance system. Malaria World Journal, 8, 1-10.

Spitzen, J., Koelewijn, T., Mukabana, W. R., \& Takken, W. (2016). Visualization of house-entry behaviour of malaria mosquitoes. Malaria Journal, 15(1), 1-10.

Spitzen, J., Koelewijn, T., Mukabana, W. R., \& Takken, W. (2017). Effect of insecticide-treated bed nets on house-entry by malaria mosquitoes: the flight response recorded in a semi-field study in Kenya. Acta Tropica, 172, 180-185.

Spitzen, J., Ponzio, C., Koenraadt, C. J. M., Pates Jamet, H. V., \& Takken, W. (2014). Absence of close-range excitorepellent effects in malaria mosquitoes exposed to deltamethrin-treated bed nets. American Journal of Tropical Medicine and Hygiene, 90(6), 1124-1132.

Spitzen, J., Smallegange, R. C., \& Takken, W. (2008b). Effect of human odours and positioning of $\mathrm{CO}_{2}$ release point on trap catches of the malaria mosquito Anopheles gambiae sensu stricto in an olfactometer. Physiological Entomology, 33(2), 116-122.

Spitzen, J., Spoor, C. W., Grieco, F., ter Braak, C., Beeuwkes, J., van Brugge, S. P., Kranenbarg, S., Noldus, L. P., van Leeuwen, J. L., \& Takken, W. (2013). A 3D analysis of flight behavior of Anopheles gambiae sensu stricto malaria mosquitoes in response to human odor and heat. PLoS One, 8(5), e62995.

Spitzen, J., Spoor, C. W., Kranenbarg, S., Beeuwkes, J., Grieco, F., Noldus, L. P. J. J., van Leeuwen, J. L., \& Takken, W. (2008a). Track3D: Visualization and flight analysis of Anopheles gambiae s.s. mosquitoes. Paper presented at Measuring Behavior. 6th International Congress on Measures and Techniques on Behavioral Research, Maastricht, The Netherlands.

Steketee, R. W., \& Campbell, C. C. (2010). Impact of national malaria control scale-up programmes in Africa: magnitude and attribution of effects. Malaria Journal, 9, 299.

Stewart, F. J., Baker, D. A., \& Webb, B. (2010). A model of visual-olfactory integration for odour localisation in free-flying fruit flies. Journal of Experimental Biology, 213(11), 1886-1900.

Stone, C. M., Chitnis, N., \& Gross, K. (2016). Environmental influences on mosquito foraging and integrated vector management can delay the evolution of behavioral resistance. Evolutionary Applications, 9(3), 502-517.

Stone, C. M., Tuten, H. C., \& Dobson, S. L. (2013). Determinants of male Aedes aegypti and Aedes polynesiensis (diptera: Culicidae) response to sound: Efficacy and considerations for use of sound traps in the field. Journal of Medical Entomology, 50(4), 723-730.

Straw, A. D., Branson, K., Neumann, T. R., \& Dickinson, M. H. (2011). Multi-camera real-time threedimensional tracking of multiple flying animals. Journal of The Royal Society Interface, 8(56), 395-409.

Strode, C., Donegan, S., Garner, P., Enayati, A. A., \& Hemingway, J. (2014). The impact of pyrethroid resistance on the efficacy of insecticide-treated bed nets against African anopheline mosquitoes: systematic review and meta-analysis. PLoS Medicine, 11(3), e1002619.

Suh, E., Choe, D. H., Saveer, A. M., \& Zwiebel, L. J. (2016). Suboptimal larval habitats modulate oviposition of the malaria vector mosquito Anopheles coluzzii. PLoS One, 11(2), e0149800.

Sutcliffe, J. F. (1987). Distance orientation of biting flies to their hosts. Insect Science and its Application, 8, 611-619.

Sutcliffe, J. F., \& Colborn, K. L. (2015). Video studies of passage by Anopheles gambiae mosquitoes through holes in a simulated bed net: effects of hole size, hole orientation and net environment. Malaria Journal, 14, 199.

Sutcliffe, J. F., Ji, X., \& Yin, S. (2017). How many holes is too many? A prototype tool for estimating mosquito entry risk into damaged bed nets. Malaria Journal, 16(1), 304.

Sutcliffe, J. F., \& Yin, S. (2014). Behavioural responses of females of two anopheline mosquito species 


\section{References}

to human-occupied, insecticide-treated and untreated bed nets. Malaria Journal, 13, 294.

Syed, Z., \& Leal, W. S. (2007). Maxillary palps are broad spectrum odorant detectors in Culex quinquefasciatus. Chemical Senses, 32(8), 727-738.

Takken, W. (1991). The role of olfaction in host-seeking of mosquitoes - a review. Insect Science and its Application, 12(1-3), 287-295.

Takken, W. (2002). Do insecticide-treated bednets have an effect on malaria vectors? Tropical Medicine and International Health, 7(12), 1022-30.

Takken, W., Dekker, T., \& Wijnholds, Y. G. (1997). Odor-mediated flight behavior of Anopheles gambiae Giles sensu stricto and $A$. stephensi Liston in response to $\mathrm{CO}_{2}$, acetone, and 1-octen3-ol (Diptera: Culicidae). Journal of Insect Behavior, 10(3), 395-407.

Takken, W., \& Knols, B. G. J. (1990). Flight behaviour of Anopheles gambiae Giles (Diptera: Culicidae) in response to host stimuli: a windtunnel study. Paper presented at the Proceedings Experimental and Applied Entomology, N.E.V., Amsterdam.

Takken, W., \& Knols, B. G. J. (1999). Odor-mediated behavior of Afrotropical malaria mosquitoes. Annual Review of Entomology, 44, 131-157.

Takken, W., \& Knols, B. G. J. (2010). Olfaction in vector-host interactions (Vol. 2). Wageningen: Wageningen Academic Publishers.

Takken, W., Smallegange, R. C., Vigneau, A. J., Johnston, V., Brown, M., Mordue-Luntz, A. J., \& Billingsley, P. F. (2013). Larval nutrition differentially affects adult fitness and Plasmodium development in the malaria vectors Anopheles gambiae and Anopheles stephensi. Parasites \& Vectors, 6(1), 345

Takken, W., van Loon, J. J. A., \& Adam, W. (2001). Inhibition of host-seeking response and olfactory responsiveness in Anopheles gambiae following blood feeding. Journal of Insect Physiology, 47(3), 303-310.

Takken, W., \& Verhulst, N. O. (2013). Host preferences of blood-feeding mosquitoes. Annual Review of Entomology, 58, 433-453.

Tauxe, Genevieve M., MacWilliam, D., Boyle, Sean M., Guda, T., \& Ray, A. (2013). Targeting a dual detector of skin and $\mathrm{CO}_{2}$ to modify mosquito host seeking. Cell, 155(6), 1365-1379.

Thoen, M. P. M., Kloth, K. J., Wiegers, G. L., Krips, O. E., Noldus, L. P. J. J., Dicke, M., \& Jongsma, M. A. (2016). Automated video tracking of thrips behavior to assess host-plant resistance in multiple parallel two-choice setups. Plant Methods, 12(1).

Thomas, M. B., Godfray, H. C. J., Read, A. F., van den Berg, H., Tabashnik, B. E., van Lenteren, J. C., Waage, J. K., \& Takken, W. (2012). Lessons from Agriculture for the Sustainable Management of Malaria Vectors. PLoS Medicine, 9(7), e1001262.

Tungu, P., Magesa, S., Maxwell, C., Malima, R., Masue, D., Sudi, W., Myamba, J., Pigeon, O., \& Rowland, M. (2010). Evaluation of PermaNet 3.0 a deltamethrin-PBO combination net against Anopheles gambiae and pyrethroid resistant Culex quinquefasciatus mosquitoes: an experimental hut trial in Tanzania. Malaria Journal, 9, 21.

Turley, A. P., Moreira, L. A., O’Neill, S. L., \& McGraw, E. A. (2009). Wolbachia infection reduces bloodfeeding success in the dengue fever mosquito, Aedes aegypti. PLoS Neglected Tropical Diseases, 3(9), e516.

Turner, S. L., Li, N., Guda, T., Githure, J., Cardé, R. T., \& Ray, A. (2011). Ultra-prolonged activation of $\mathrm{CO}_{2}$-sensing neurons disorients mosquitoes. Nature, 474(7349), 87-91.

Tusting, L. S., Ippolito, M. M., Willey, B. A., Kleinschmidt, I., Dorsey, G., Gosling, R. D., \& Lindsay, S. W. (2015). The evidence for improving housing to reduce malaria: A systematic review and meta-analysis. Malaria Journal, 14(1), 209.

Tusting, L. S., Willey, B., \& Lines, J. (2016). Building malaria out: improving health in the home. Malaria Journal, 15(1), 1-3. 
van Breugel, F., Riffell, J., Fairhall, A., \& Dickinson, Michael H. (2015). Mosquitoes use vision to associate odor plumes with thermal targets. Current Biology, 25(16), 2123-2129.

Vanickova, L., Canale, A., \& Benelli, G. (2017). Sexual chemoecology of mosquitoes (Diptera, Culicidae): Current knowledge and implications for vector control programs. Parasitology International, 66(2), 190-195.

Verhulst, N. O., Loonen, J. A., \& Takken, W. (2013). Advances in methods for colour marking of mosquitoes. Parasites \& Vectors, 6(1), 200.

Verhulst, N. O., Qiu, Y. T., Beijleveld, H., Maliepaard, C., Knigths, D., Schulz, S., Berg-Lyons, D., Lauber, C. L., Verduijn, W., Haasnoot, G. W., Mumm, R., Bouwmeester, H. J., Claas, F. H. J., Dicke, M., van Loon, J. J. A., Takken, W., Knight, R., \& Smallegange, R. C. (2011). Human skin microbiota affects attractiveness to malaria mosquitoes. PLoS One, 6(12), e28991.

Vickers, N. J., \& Baker, T. C. (1994). Reiterative responses to single strands of odor promote sustained upwind flight and odor source location by moths. Proceedings of the National Academy of Sciences, 91, 5756-5760.

Visser, J. H., \& Avé, D. A. (1978). General green leaf volatiles in the olfactory orientation of the Colorado beetle, Leptinotarsa decemlineata. Entomologia Experimentalis et Applicata, 24, 538-549.

Vogel, G. (2013). The forgotten malaria. Science, 342(6159), 684-687.

Vogels, C. B. F., Fros, J. J., Pijlman, G. P., van Loon, J. J. A., Gort, G., \& Koenraadt, C. J. M. (2017). Virus interferes with host-seeking behaviour of mosquito. Journal of Experimental Biology, 220(19), 3598-3603.

von Seidlein, L., Kekulé, A. S., \& Strickman, D. (2017). Novel Vector control approaches: The future for prevention of Zika virus transmission? PLoS Medicine, 14(1), e1002219.

Wang, G. R., Qiu, Y. T., Lu, T., Kwon, H. W., Pitts, R. J., van Loon, J. J. A., Takken, W., \& Zwiebel, L. J. (2009). Anopheles gambiae TRPA1 is a heat-activated channel expressed in thermosensitive sensilla of female antennae. European Journal of Neuroscience, 30(6), 967-974.

Wang, J., Lin, C., Ji, L., \& Liang, A. (2012). A new automatic identification system of insect images at the order level. Knowledge-Based Systems, 33, 102-110.

Wang, R., Hu, C., Fu, X., Long, T., \& Zeng, T. (2017). Micro-Doppler measurement of insect wing-beat frequencies with W-band coherent radar. Scientific Reports, 7(1), 1396.

Wanzirah, H., Tusting, L. S., Arinaitwe, E., Katureebe, A., Maxwell, K., Rek, J., Bottomley, C., Staedke, S. G., Kamya, M., Dorsey, G., \& Lindsay, S. W. (2015). Mind the gap: House structure and the risk of malaria in Uganda. PLoS One,10(1), e0117396.

Warburg, A., Faiman, R., Shtern, A., Silberbush, A., Markman, S., Cohen, J. E., \& Blaustein, L. (2011). Oviposition habitat selection by Anopheles gambiae in response to chemical cues by Notonecta maculata. Journal of Vector Ecology, 36(2), 421-425.

Warren, B., Gibson, G., \& Russell, I. J. (2009). Sex recognition through midflight mating duets in Culex mosquitoes is mediated by acoustic distortion. Current Biology, 19(6), 485-491.

Webster, B. (2012). The role of olfaction in aphid host location. Physiological Entomology, 37(1), 10-18.

Webster, B., Lacey, E. S., \& Cardé, R. T. (2015). Waiting with bated breath: opportunistic orientation to human odor in the malaria mosquito, Anopheles gambiae, is modulated by minute changes in carbon dioxide concentration. Journal of Chemical Ecology, 41(1), 59-66.

Weseka, J. W., Brogdon, W. G., Hawley, W. A., \& Besansky, N. J. (1998). Flight tone of field-collected populations of Anopheles gambiae and An. arabiensis (Diptera: Culicidae). Physiological Entomology, 23, 289-294.

White, B. J., Collins, F. H., \& Besansky, N. J. (2011). Evolution of Anopheles gambiae in relation to humans and malaria. Annual Review of Ecology, Evolution, and Systematics, 42(1), 111-132. 


\section{References}

White, G. B. (2006). Terminology of Insect Repellents. In M. F. Debboun, S.P.; Strickman, D. (Ed.), Insect repellents: principles, methods and uses (pp. 31-46). Boca Raton: CRC Press.

White, N. J., Pukrittayakamee, S., Hien, T. T., Faiz, M. A., Mokuolu, O. A., \& Dondorp, A. M. (2014). Malaria. The Lancet, 383(9918), 723-735.

Williams, C. R., Bergbauer, R., Geier, M., Kline, D. L., Bernier, U. R., Russell, R. C., \& Ritchie, S. A. (2006). Laboratory and field assessment of some kairomone blends for host-seeking Aedes aegypti. Journal of the American Mosquito Control Association, 22(4), 641-647.

Woltring, H. J. (1980). Planar control in multi-camera calibration for 3-D gait studies. Journal of Biomechanics, 13(1), 39-48.

Wondwosen, B., Birgersson, G., Seyoum, E., Tekie, H., Torto, B., Fillinger, U., Hill, S. R., \& Ignell, R. (2016). Rice volatiles lure gravid malaria mosquitoes, Anopheles arabiensis. Scientific Reports, 6, 37930.

World Health Organization, WHO. (2005). Guidelines for laboratory and field testing of long-lasting insecticidal mosquito nets (pp. 18). Geneva.

World Health Organization, WHO. (2006). Guidelines for testing mosquito adulticides for indoor residual spraying and treatment of mosquito nets (pp. 70). Geneva.

World Health Organization, WHO. (2012). Handbook for integrated vector management.

World Health Organization, WHO. (2015). Action and Investment to defeat Malaria 2016-2030. For a Malaria-Free World (R. B. M. partnership, Trans.) (pp. 97). Geneva.

World Health Organization, WHO. (2016a). World malaria report 2016 (pp. 186). Geneva.

World Health Organization, WHO. (2016b). Implications of insecticide resistance for malaria vector control (pp. 4). Geneva.

World Health Organization, WHO. (2017a). World malaria report 2017 (pp. 196). Geneva.

World Health Organization, WHO. (2017b). Global vector control response 2017-2030. Geneva.

Yang, H. P., Ma, C. S., Wen, H., Zhan, Q. B., \& Wang, X. L. (2015). A tool for developing an automatic insect identification system based on wing outlines. Scientific Reports, 5, 12786.

Young, S. J., Gunning, R. V., \& Moores, G. D. (2006). Effect of pretreatment with piperonyl butoxide on pyrethroid efficacy against insecticide-resistant Helicoverpa armigera (Lepidoptera: Noctuidae) and Bemisia tabaci (Sternorrhyncha: Aleyrodidae). Pest Management Science, 62(2), 114-119.

Yuval, B. (1992). The other habit: sugar feeding by mosquitoes. Bulletin of the Society for Vector Ecology, 17(2), 150-156.

Zermoglio, P. F., Robuchon, E., Leonardi, M. S., Chandre, F., \& Lazzari, C. R. (2017). What does heat tell a mosquito? Characterization of the orientation behaviour of Aedes aegypti towards heat sources. Journal of Insect Physiology, 100, 9-14.

Zhou, L. X. (2006). Long-term record of atmospheric $\mathrm{CO}_{2}$ and stable isotopic ratios at Waliguan Observatory: Seasonally averaged 1991-2002 source/sink signals, and a comparison of 19982002 record to the 11 selected sites in the Northern Hemisphere. Global Biogeochemical Cycles, 20(2), GB2001. 

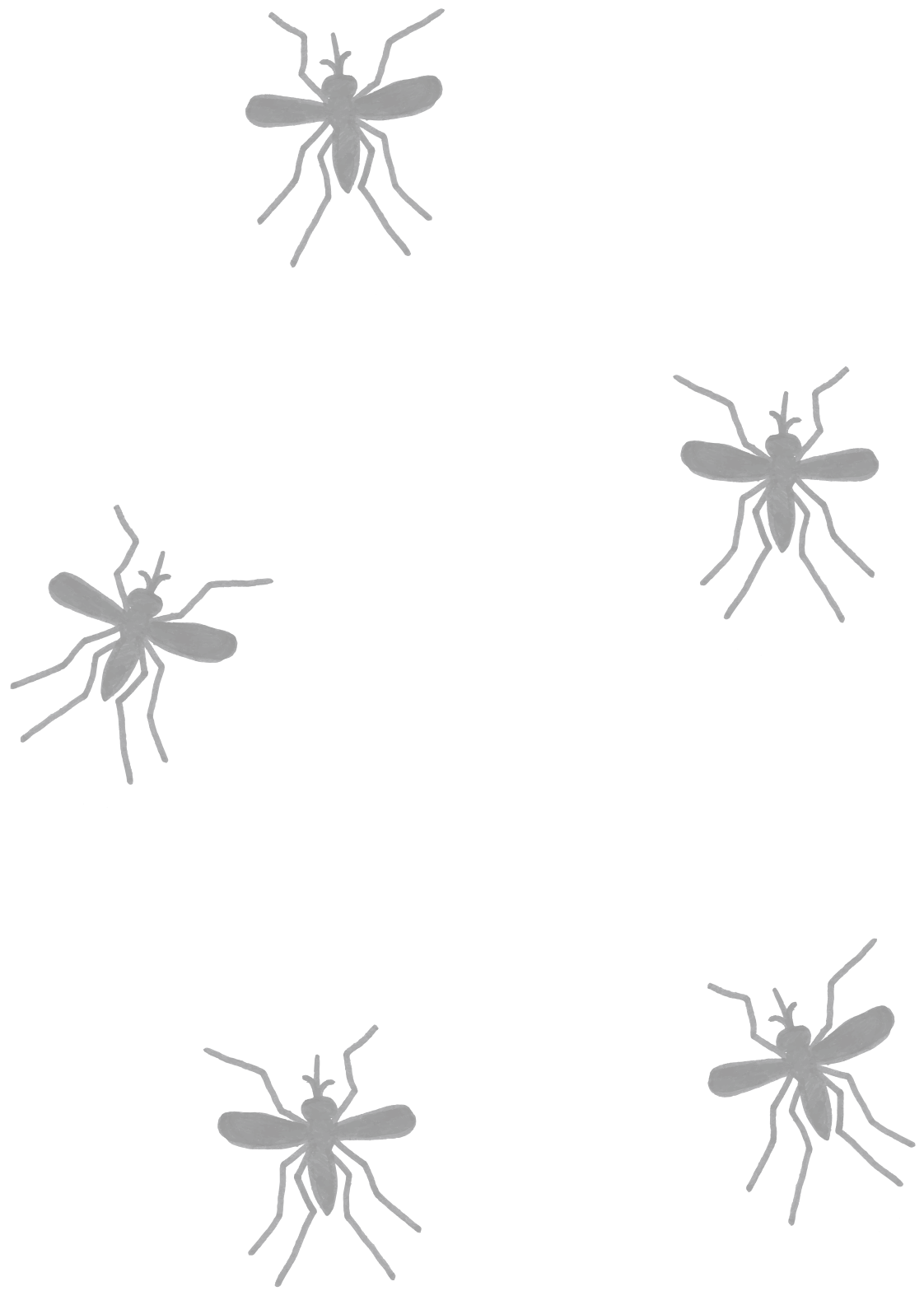


\section{Summary}

Among the mosquitoes that act as vectors of infectious diseases, the genus Anopheles compiles 41 species considered dominant in transmitting the malaria parasite Plasmodium to humans. The World Health Organisation reported an estimated number of 216 million human malaria cases in 2016, with approximately 445,000 deaths worldwide. Although great reductions in disease mortality and morbidity were achieved with vector-control tools such as long-lasting-insecticidetreated nets (LLIN's) and indoor residual spraying (IRS), these tools currently lose their efficacy due to behavioural and physiological resistance in the mosquitoes. Alternative control methods are required. Many of these methods aim to interrupt mosquito-host interactions, e.g. by the use of odour-baited traps, push-pull systems, or house improvements. For the innovation and successful implementation of alternative, sustainable tools it is necessary to understand mosquito behaviour in detail so that interventions can be made more precisely and effectively.

In this thesis, I focus on the flight behaviour of one of the main vectors of malaria parasites, the mosquito Anopheles gambiae Giles sensu stricto, while exposed to specific host cues. The aim of my study was to unravel behavioural characteristics of host-seeking mosquitoes, in support of the development of more effective vector control interventions. In order to achieve this, an automated tracking tool was developed with which mosquitoes could be accurately tracked during their hostseeking flight.

Chapter 2 provides a review that focuses on the tracking of mosquitoes in space in order to elucidate fundamental aspects of their behaviour across different phases of their life history. An overview of tools used for behavioural tracking of mosquitoes and their technical complexities is provided. Automated tracking techniques have contributed to our understanding of how the multimodal integration of (host-) cues plays a role in source finding of mosquitoes. The tools have provided information on attraction or repellent modes by studying mosquito behaviour around insecticidetreated surfaces such as bed nets, or around odour-baited traps. The review discusses how the knowledge obtained can support successful implementations of vector-control strategies in the field.

The specific role of carbon dioxide $\left(\mathrm{CO}_{2}\right)$ in relation to skin emanations was studied in Chapter 3. In an olfactometer, I tested trap entry responses of female An. gambiae mosquitoes to either homogeneous- or turbulent $\mathrm{CO}_{2}$ plumes at two different positions 
from the trap entrance. Anopheles gambiae was deterred from entering a trap with $\mathrm{CO}_{2}$ as the only kairomone, when presented as an homogeneous or turbulent plume. This effect was completely overcome by the addition of skin emanations to the $\mathrm{CO}_{2}$ plume, with a high proportion of mosquitoes found in the trap with skin emanations. Rearrangement of the position of the turbulent $\mathrm{CO}_{2}$ source, so that it was $5 \mathrm{~cm}$ downwind of the trap entrance, overcame the deterrent effect of $\mathrm{CO}_{2}$. Carbon dioxide alone, however, did not elicit higher proportions caught than clean air. Further studies with the $\mathrm{CO}_{2}$ source positioned $5 \mathrm{~cm}$ downwind of the trap entrance showed that skin emanations alone resulted in fewer mosquitoes entering the trap than $\mathrm{CO}_{2}+$ skin emanations. It is concluded that $\mathrm{CO}_{2}$ is a poor kairomone when offered alone and that its presence in the plume at the trap entrance deters mosquitoes from entering. By contrast, when positioned just downwind of the trap entrance, $\mathrm{CO}_{2}$ appears to guide mosquitoes to the vicinity of the trap, where skin emanations then become the principle attractant, causing mosquito trap entry response. The results have implications for the design of odour-baited traps for this mosquito species.

Female mosquitoes use odour and heat as cues to navigate to a suitable landing site on their blood host. How these cues affect flight behaviour and modulate anemotactic responses was studied in Chapter 4. In-flight behavioural responses of female mosquitoes to human odour and heat were studied in a wind tunnel using a newly developed automated tracking system. Flightpath characteristics were quantified in three dimensions. With wind $(20 \mathrm{~cm} / \mathrm{s})$ as the only stimulus (control), short and close-to-straight upwind flights were recorded. With heat alone, flights were similarly short and direct. The presence of human odour, by contrast, caused prolonged and highly convoluted flight patterns. The combination of odour + heat resulted in longer flights with more landings on the source than to either cue alone. Flight speed was greatest (mean groundspeed $27.2 \mathrm{~cm} / \mathrm{s}$ ) for odour + heat. Odour alone resulted in decreased flight speed when mosquitoes arrived within $30 \mathrm{~cm}$ of the source whereas mosquitoes exposed to odour + heat maintained a high flight speed while flying in the odour plume, until they arrived within $15 \mathrm{~cm}$ of the source. Human odour evoked an increase in crosswind flights with an additive effect of heat at close range $(<15 \mathrm{~cm})$ to the source. This was found for both horizontal and vertical flight components. However, mosquitoes nevertheless made upwind progress when flying in this odour plume with heat as extra stimulus, suggesting that mosquitoes scan their environment intensively while they progress upwind towards their host. These observations helped to improve the efficacy of trapping systems for malaria mosquitoes by (1) optimizing the site of odour release relative to trap entry and (2) adding a heat source which enhances a landing response or trap entry. 
The tracking tools that were initially installed to analyse flight behaviours in wind tunnels were partly dismantled and brought to Kenya to study the house-entry behaviour of malaria mosquitoes in a semi-field setting. In Chapter 5 the approach of female mosquitoes towards the eave of an experimental house is described and analysed. Mosquitoes were released in a large screenhouse and lured to the experimental house by releasing a standardized synthetic odour blend $+\mathrm{CO}_{2}$ from under an untreated bed net.

The majority of host-seeking An. gambiae approached the house with a flight altitude at eave level, arriving within a horizontal arc of $180^{\circ}$. Fifty-five per cent of mosquitoes approaching a house did not enter or made multiple attempts before passing through the eave. During approach, mosquitoes greatly reduced their speed and the flight paths became more convoluted. As a result, mosquitoes that passed through the eave spent more than $80 \%$ of the observed time within $30 \mathrm{~cm}$ of the eave. Mosquitoes that exited the eave departed at eave level and followed the edge of the roof $(12.5 \%)$ or quickly re-entered after exiting $(9.6 \%)$.

The study shows that host-seeking mosquitoes, when entering a house, approach the eave in a wide angle to the house at eave level. Less than $25 \%$ of approaching mosquitoes entered the house without interruption, whereas $12.5 \%$ of mosquitoes that had entered left the house again within the four hours' time of observation. Advances in tracking techniques open a new array of possibilities to test and improve household interventions. This may be done, for example, by studying the effect of (partial) house improvements or the installation of eave tubes on mosquito house entry behaviour.

Insecticide-treated nets are currently a major tool to reduce malaria transmission. Their degree of repellence affects contact of the mosquito with the net, but may also influence the mosquito's entry into the house. Using the same experimental setup as in Chapter 5, in Chapter 6 we investigated the response of host-seeking malaria mosquitoes approaching the eave and compared house entry- and exit rates in relation to the presence of different insecticide-treated bed nets (ITNs) with an untreated net. Again, mosquitoes were lured towards the house by dispensing a synthetic host-odour blend from within the net in the house. Complementary WHO bioassays revealed that the treated nets caused high knock-down- and mortality responses in the mosquitoes tested.

The proportion of mosquitoes that came into view of the cameras and subsequently entered the house did not differ between treated nets and the untreated net. Treated nets did not affect proportions of mosquitoes that exited the house and departed 
from view around the eave. However, the percentage of house-leaving and reentering mosquitoes when an insecticide- treated net was present, was lower than in the presence of an untreated net.

The results indicate that there was no spatial repellent effect from pyrethroid-treated nets that influences house entry at eave level. It is argued that the toxic effect of treated bed nets resulted in a reduced number of mosquitoes re-entering the house, which may affect malaria transmission in neighbouring, unprotected houses.

Close-range flight responses of mosquitoes exposed to LLINs in combination with human host cues are described in Chapter 7 . The flight behaviour and contact time of insecticide-resistant and susceptible malaria mosquitoes approaching deltamethrintreated nets was recorded inside a wind tunnel. After exposure to the bed nets, the observed behaviour was linked to the resulting health status of the mosquitoes (dead or alive) using comparisons between outcomes from 'free-flight' assays and standard WHO bioassays.

There was no difference in response time, latency time to reach the net or spatial distribution in the wind tunnel between treatments. Unaffected resistant mosquitoes spent less time close to $(<30 \mathrm{~cm})$ treated nets. Nettings that caused high knock down or mortality in standard WHO assays evoked significantly less mortality in the wind tunnel; there was no excito-repellent effect in mosquitoes making contact with the nettings in free-flight. This Chapter demonstrates a new approach to understanding mosquito behaviour near insecticidal nets. The methodology links free-flight behaviour to mosquito health status upon exposure to nets. The results show that behavioural assays can provide important insights for the evaluation of insecticidal effects on disease vectors.

This thesis provides answers to fundamental and applied aspects that play a role during host seeking of female mosquitoes. The main findings are discussed and placed into future perspective in Chapter $\mathbf{8}$. The research confirmed the crucial role of $\mathrm{CO}_{2}$ and its function is a key target for further exploration to manipulate or disrupt the host-seeking process of blood-feeding mosquitoes. The development of automated tracking techniques contributed to insights on the approach of mosquitoes to hosts rather than measuring end points only. By doing so, it was found that heat is vital for malaria mosquitoes to induce landings near an odour source, but that odour alone can evoke typical highly convoluted flight paths that were observed in both wind tunnel studies as well as in the semi-field in Africa by studying mosquito house entry behaviour. The presence of insecticide-treated nets inside a house did not repel mosquitoes, however, their toxic effect seems to affect the number of mosquitoes that re-enter such houses. By recording mosquitoes exposed to insecticide-treated 
nets in a wind tunnel, their free-flight behaviour could be linked to the mosquito health status and related to the contact time with the treated materials.

Detailed analyses of mosquito behaviours as presented in this thesis can be exploited to support the innovation of vector control tools and measure their effectiveness. Direct and indirect applications have successfully been installed in the field. For example, the development of odour baited traps that separated the $\mathrm{CO}_{2}$ release point from the odour blend, or by the addition of heat to enhance mosquito landings. Insights on house-entry behaviour and mosquito responses to bed nets supports successful implementation of push-pull systems, installation of eave tubes or houseimprovement operations to reduce malaria transmission. 


\section{Samenvatting}

In Nederland vinden mensen muggen vooral vervelend omdat hun gezoem ze wakker houdt en de beet van vrouwtjes een nare jeuk kan veroorzaken. Deze -betrekkelijk geringe- overlast staat in schril contrast met de rol van muggen als overbrenger ('vector') van infectieziekten. Binnen het muggengeslacht Anopheles staan 41 muggensoorten bekend als belangrijke vector van de malariaparasiet Plasmodium naar de mens. Infectie met Plasmodium kan de ziekte malaria veroorzaken. Volgens de Wereldgezondheidsorganisatie (WHO) waren er in het jaar 2016 wereldwijd zo'n 216 miljoen gevallen van malaria, met ongeveer 445.000 sterfgevallen tot gevolg.

De laatste 15 jaar is het aantal ziekte- en sterfgevallen door malaria gehalveerd door onder meer het gebruik van geïmpregneerde klamboes en het binnenshuis gebruik van insecticiden. Desondanks zijn de huidige bestrijdingsmaatregelen niet voldoende om een verdere afname te verwezenlijken. Dit is ten dele het gevolg van gedragsaanpassingen van de mug en resistentie van de mug tegen insecticiden, en van de parasiet tegen medicijnen. De ontwikkeling van alternatieve bestrijdingsmethoden is daarom noodzakelijk.

Veel methoden richten zich op het verstoren van de interactie tussen mug en mens. Voorbeelden zijn het gebruik van geurvallen, het verbeteren van woningen of 'pushpull'-systemen. Die laatste maken gebruik van geurstoffen die muggen afstoten of juist aantrekken. Voor innovatie en het succesvol implementeren van alternatieve en duurzame bestrijdingsmethoden is het noodzakelijk het gedrag van muggen tot in detail te begrijpen, zodat de interventies op maat gemaakt kunnen worden en efficiënt kunnen worden ingezet.

Dit proefschrift focust op het vlieggedrag van één van de belangrijkste vectoren van de malariaparasiet, de Afrikaanse malariamug Anopheles gambiae Giles sensu stricto, tijdens de blootstelling aan specifieke, gastheergerelateerde gedragsprikkels. Het doel van dit onderzoek was het vlieggedrag te ontrafelen van muggen die een menselijke gastheer zoeken. De resultaten van dit onderzoek kunnen als basis dienen voor het ontwikkelen van effectieve vector-bestrijdingsmethoden. Voor dit onderzoek is een proefopstelling ontwikkeld die het mogelijk maakt het vlieggedrag van muggen in het donker te filmen en nauwkeurig te analyseren. Hoofdstuk 1 beschrijft de verdere onderbouwing en achtergrond van het uitgevoerde onderzoek. Hoofdstuk 2 betreft een overzichtsartikel. Dit hoofdstuk zet allereerst uiteen hoe gedragsstudies aan muggen, tijdens de verschillende levensfasen, bijdragen aan het begrijpen van hun biologie. Het bespreekt de ontwikkeling van diverse 
observatiemethoden, inclusief de technische uitdagingen die hierbij een rol spelen. Omdat muggen veelal 's nachts actief zijn, een grillig vliegpatroon hebben en klein zijn, hebben geautomatiseerde registratiesystemen ('tracking tools') bijgedragen aan het vastleggen van hun vlieggedrag. Tegelijkertijd kunnen we met deze registratiesystemen ook bestuderen hoe de mug verschillende prikkels integreert tijdens het lokaliseren van de bron van die prikkels. Het kan daarbij gaan om gastheergerelateerde prikkels, zoals lichaamswarmte en humane geurstoffen, en niet-gastheergerelateerde prikkels, zoals licht. Dankzij deze methoden weten we nu veel meer over aantrekkende, of juist afstotende eigenschappen die bijdragen aan het succes van interventiemethoden zoals geïmpregneerde klamboes of geurvallen. Het hoofdstuk beschrijft hoe vergaarde kennis verder kan bijdragen aan de implementatie van bestrijdingsmethoden in het veld.

Vrouwtjesmuggen komen op koolstofdioxide $\left(\mathrm{CO}_{2}\right)$ af, maar desondanks vliegen ze zelden in iemands mond, terwijl dat de bron van een verhoogde $\mathrm{CO}_{2}$-concentratie is. Hoofdstuk 3 beschrijft keuzeproeven in een reukmeter die inzicht gaven in de specifieke rol van $\mathrm{CO}_{2}$ in relatie tot humane geurstoffen afkomstig van de huid. Deze keuzeproeven testten het effect van zowel homogene als turbulente $\mathrm{CO}_{2}$-pluimen op twee verschillende posities ten opzichte van een valopening. Anopheles gambiaemuggen werden afgestoten van de valopening als $\mathrm{CO}_{2}$ de enige aanwezige lokstof was. Dit effect werd volledig opgeheven wanneer huidgeur (op een gedragen sok) werd toegevoegd in de val. Een turbulente $\mathrm{CO}_{2}$-bron die $5 \mathrm{~cm}$ vóór de valopening werd geplaatst nam het afstotende effect ook weg. De combinatie van turbulente $\mathrm{CO}_{2}$ vóór de valopening en huidgeur ín de val leverde hogere vangsten op dan in vallen waarbij beide lokstoffen in de val werden geplaatst. Het gunstige effect van deze combinatie was nog duidelijker te zien wanneer deze werd getest tegen een val met alleen huidgeur. De conclusie is dat $\mathrm{CO}_{2}$ op kortere afstand voor deze mug op zichzelf een zwakke signaalstof is, en bij hoge concentraties dicht bij de bron muggen kan afstoten. Echter, wanneer $\mathrm{CO}_{2}$ wordt gescheiden van huidgeuren en benedenwinds wordt losgelaten, kan het muggen in de buurt van valopeningen brengen. Vanaf daar krijgen huidgeuren een dominante functie in de aantrekking op muggen met als gevolg verhoogde muggenvangsten. De resultaten van deze studie zijn van belang voor het ontwerp van geurvallen voor malariamuggen.

Voor het navigeren naar een geschikte landingsplek op de gastheer gebruiken muggen zowel geur- als warmteprikkels. Hoe deze prikkels de precisie van het vlieggedrag van vrouwtjesmuggen beïnvloeden, is onderwerp van Hoofstuk 4. Dit onderzoek maakte gebruik van een speciaal hiervoor gebouwd klimaatbeheersingssysteem, gekoppeld aan een windtunnel voorzien van camera's en infraroodlampen. In 
teamverband ontwikkelden we software die automatische detectie en reconstructie van het afgelegde vliegtraject in drie dimensies mogelijk maakt. Met deze proefopstelling kunnen we het gedrag van nacht-actieve muggen kwantificeren en tot in detail analyseren. De aanwezigheid van een humane geurbron (een gedragen sok) resulteerde in verlengde vliegactiviteit en intensievere zigzag-vliegpatronen vergeleken met blootstelling aan slechts één warmtebron of alleen gefilterde lucht. De combinatie van geur en warmte leidde tot een verlengde vliegduur en significant meer landingen op de bron, vergeleken met testen met slechts één van de twee stimuli. Muggen vlogen meer dwars op de wind bij de aanwezigheid van huidgeur en het effect van warmte was vooral evident op korte afstand van de bron, bij minder dan $15 \mathrm{~cm}$. Dit effect trad op zowel voor horizontale als voor verticale vluchtcomponenten. Ondanks dit dwars op de wind vliegen kwamen muggen uiteindelijk wel dichter bij de bron in de aanwezigheid van huidgeur en warmte, wat suggereert dat ze hun omgeving intensief verkennen voordat ze een landing inzetten. De gedane observaties kunnen bijdragen aan het ontwikkelen van effectievere vangsystemen voor malariamuggen door bijvoorbeeld (1) de geurbron op een optimale afstand van de vangopening te plaatsen of (2) een warmtebron aan geurvallen toe te voegen om landingen of vangsten te verhogen.

Naast fundamentele vragen over het vlieggedrag van muggen die zich goed in het laboratorium laten testen, zijn er ook in het veld een aantal vragen bestudeerd die tot dusver letterlijk onderbelicht zijn gebleven bij gebrek aan geschikte observatietechnieken. Het onderzoek beschreven in Hoofdstuk 5 richt zich op hoe muggen de voor veel Afrikaanse huizen typische dakrandopening (eave) benaderen en passeren wanneer ze aangetrokken worden door een synthetische mix van lokstoffen en $\mathrm{CO}_{2}$. Muggen werden losgelaten in een grote gazen tentruimte met daarin een experimenteel huis, waarbij aan één kant van het huis de dakrandopening van buitenaf werd gefilmd met twee camera's, om naar binnen en buiten vliegende muggen te kunnen observeren.

Het merendeel van de gastheerzoekende An. gambiae-muggen benaderde het huis ter hoogte van de dakrandopening en vloog niet van onder via de muur omhoog richting de opening, zoals eerder vermeld was in de literatuur. Drieëntwintig procent van de muggen die het huis naderden, vlogen in één keer naar binnen. Vijfenvijftig procent van de muggen die in beeld kwamen, vlogen niet naar binnen, of deden meerdere pogingen alvorens naar binnen te vliegen via de dakrandopening. Bij muggen die naar binnen vlogen nam bij het benaderen van de opening de vliegsnelheid drastisch af, en was een meer zigzag-vliegpatroon te zien. Hierdoor werden muggen die naar binnen vlogen, voor $80 \%$ van de tijd dat ze in beeld waren aangetroffen 
in een gebied binnen $30 \mathrm{~cm}$ van de opening. Een vergelijkbaar vliegpatroon als beschreven in Hoofdstuk 4, maar omdat er bij het binnenvliegen geen landingen optraden duurden deze vliegpassages slechts enkele seconden. Muggen die weer naar buiten kwamen (12.5\% na 4 uur filmen), vlogen vlak langs de dakrand weg, een deel (9.6\%) vloog vrijwel direct opnieuw naar binnen. Deze observaties en de gebruikte technieken bieden nieuwe mogelijkheden voor het ontwikkelen en testen van bestrijdingsmaatregelen gericht op het huishouden, ontwerp en afwerking van woningen met als doel mug-menscontact te laten afnemen.

Het gebruik van geïmpregneerde klamboes wordt momenteel gezien als één van de succesvolste maatregelen om transmissie van malariaparasieten te voorkomen. De mate van afstotende eigenschappen van deze netten, als gevolg van de behandeling met chemicaliën, is van belang voor het contact dat de muggen met de (toxische) netten zullen hebben, maar heeft mogelijk ook invloed op het binnenvliegen van huizen waar de netten zijn opgehangen. Dit laatste is onderzocht in Hoofdstuk 6 , waar dezelfde onderzoeksopstelling gebruikt is als in Hoofdstuk 5, met het verschil dat er tijdens verschillende nachten een ander type geïmpregneerde klamboe in het experimentele huis was opgehangen. De ratio van naar binnen en buiten vliegende muggen werd vergeleken met de controle: een onbehandeld net. Aanvullend testten we of de gebruikte netten daadwerkelijk toxisch waren na geforceerd contact volgens de standaard WHO-toets. Het percentage muggen dat in beeld kwam en vervolgens naar binnen vloog, was gelijk bij aanwezigheid van geïmpregneerde klamboes wanneer vergeleken met het onbehandelde net. Ook het aantal muggen dat het huis uitvloog en uit beeld verdween, was in verhouding gelijk. Alleen het percentage muggen dat naar buiten vloog en meteen hierop weer terug naar binnen vloog, was hoger bij aanwezigheid van een onbehandeld net. Deze resultaten zijn een indicatie dat met pyrethroïden-behandelde klamboes geen vluchtige afstotende werking hebben en op die manier het binnenvliegen niet beïnvloeden. Echter, het toxische effect van de insecticiden vermindert het aantal muggen dat opnieuw naar binnen vliegt. Dit heeft mogelijk gevolgen voor de malariatransmissie in omliggende huizen, al dan niet in aanwezigheid van beschermende (mugwerende) maatregelen.

Hoofdstuk 7 beschrijft de effecten van geïmpregneerde klamboes op het vlieggedrag op korte afstand van deze klamboes. Dit onderzoek koppelde de vliegrespons van individuele muggen aan hun 'lot' als gevolg van het contact met de werkzame toxische stof. Zowel vatbare als insecticide-resistente muggen werden getest in een windtunnel met daarin humane geur en warmte áchter klamboes die met deltamethrine behandeld waren. De gedragingen, waaronder de duur van aanraking met de klamboe, werden gemeten en vergeleken met die bij een onbehandelde klamboe. Na iedere test, van vijf minuten per individu, werd de mug gevangen, waarna werd bijgehouden of deze na één uur 'knockout' lag en of er na 24 uur mortaliteit was opgetreden. $\mathrm{Er}$ 
was geen verschil tussen de verschillende klamboes wat betreft reactietijd, duur tot de eerste aanraking met de klamboe, of ruimtelijke verdeling van muggen in de windtunnel. Resistente muggen die na afloop van de gedragstest na 24 uur nog in leven waren, bevonden zich verhoudingsgewijs minder lang in de nabijheid $(<30$ $\mathrm{cm}$ ) van behandelde klamboes. Bij geforceerd contact (volgens de standaard WHOtoets) was de 'knockdown' en mortaliteit significant hoger dan in een windtunnel waarin muggen vrij konden vliegen. Het onderzoek toont een nieuwe aanpak om het gedrag van muggen rondom klamboes te bestuderen en kan daarmee de effectiviteit van verschillende materialen toetsen. De methodologie koppelt het gedrag van 'vrij' vliegende muggen, na blootstelling aan geïmpregneerde klamboes in combinatie met humane lokstoffen, aan hun gezondheid en overleving achteraf.

Dit proefschrift verschaft antwoorden op zowel fundamentele als toegepaste vraagstukken die een rol spelen tijdens het zoekgedrag van vrouwtjesmuggen naar een bloedmaaltijd. Hoofdstuk 8 bespreekt de belangrijkste bevindingen en plaatst deze in toekomstig perspectief. Het onderzoek onderstreept de cruciale rol van $\mathrm{CO}_{2}$. De uitwerking ervan op muggen vormt een speerpunt voor vervolgonderzoek met als doel het proces van gastheerzoeken bij muggen te verstoren of te manipuleren. De ontwikkeling van een proefopstelling en software voor automatische detectie en reconstructie van vliegbewegingen heeft bijgedragen aan inzichten over de gedragingen van nacht-actieve muggen op weg naar een zeker doel (de gastheer, of een huis) in plaats van het meten van enkel eindpunten. Hiermee is bijvoorbeeld aangetoond dat warmte van belang is voor het induceren van een landing nabij een geurbron, en dat de aanwezigheid van humane geur an sich kenmerkende zigzagvliegpatronen oplevert. De aanwezigheid van geïmpregneerde klamboes heeft geen effect op het aantal muggen dat een huis binnenvliegt; desondanks lijkt het toxische effect het aantal muggen dat opnieuw naar binnen vliegt, te verminderen. Het filmen van muggen tijdens blootstelling aan geïmpregneerde klamboes in een windtunnel maakte het mogelijk het geobserveerde gedrag te koppelen aan het 'lot' van de mug in relatie tot de duur van het contact met de behandelde materialen.

Gedetailleerde analyses van het gedrag van muggen, zoals beschreven in dit proefschrift, kunnen gebruikt worden in de innovatie van bestrijdingsmaatregelen, of bij de evaluatie daarvan. Er zijn inmiddels zowel directe als indirecte toepassingen in het veld. Zo wordt bijvoorbeeld bij de ontwikkeling van geurvallen de $\mathrm{CO}_{2}$-bron gescheiden van het synthetisch geurmengsel, of wordt er warmte toegevoegd om extra landingen te generen. Inzichten in hoe muggen een huis binnenvliegen en in hun gedragingen rondom klamboes kunnen bijdragen aan succesvolle implementatie van 'push-pull'-systemen, installatie van ventilatiebuizen met mugwerende middelen, of verbetering van het ontwerp en de bouw van huizen, dit alles met als doel de transmissie van malaria te blokkeren. 


$$
\hat{\widehat{A}} \widehat{\hat{n}}
$$




\section{Acknowledgements}

The longer you work, the longer the list of people to thank. I'm not sure where to start to thank all the people that contributed one way or the other to this thesis. When I started working at the Laboratory of Entomology in 2002, I had no intention to start (or finish) a PhD thesis, but life can be unpredictable. So let's start with the people that work, or used to work at Ento, over the last 16 years. Thank you all for being great colleagues!

There's only a few people who like mosquitoes. Why would somebody want to work with these insects? Some people argue that mosquitoes make good nature conservationists by deterring humans from vulnerable wildlife areas, but the majority of mankind hates them. Which is understandable, given the nuisance they cause and their ability to spread infectious diseases such as malaria. It is a characteristic of biologists that they can develop an interest for basically every organism they study through understanding their physiology, ecology or behaviour. With this argument I convinced Arnold van Huis and Willem Takken during my job interview. They thought that I was suitable for the position of 'technician tropical entomology'. Gratefully, Joop van Lenteren, at that time head of the Laboratory of Entomology, offered me one of the few available permanent positions, and so I started, without knowing anything about insects, besides the knowledge that spiders have a pair of extra legs. Both Arnold and Willem stimulated me to conduct my own research. The pile of 'mosquito-topics' was high and expanding, whereas Arnold's students mostly worked abroad, especially after the Callosobruchus and Uscana rearing left the building. Kindly, Arnold gave me more time to work on mosquitoes, as long as I continued co-organizing the course 'ecological aspects of bio-interactions'. Thank you Arnold and Willem for giving me this opportunity. Willem, you have always been a great motivator to me to work on the behavioural aspects of medical entomology. I will miss that. We get to see you less often in the lab, but you will get your well deserved time in Olst to enjoy your great house and garden project. Another thanks to Willem for pointing out the farmhouse where Annemarieke and I live in now. Without finding this, we most likely would have looked abroad...

Marcel, you got involved at the final stage during my thesis writing. It's true what all the other PhD's have said. You reply extremely fast to manuscript drafts, with a great eye for detail. Your enthusiasm across all the different entomology topics is just crazy, but only to admire. 
Yu-Tong en Renate, wat leuk dat ik mee naar de Gambia kon! Renate, het $\mathrm{CO}_{2}$ artikel is inmiddels knetteroud, maar staat voorin dit proefschrift. Nog altijd relevant. En het 'sugar-fermented yeast' artikel staat bovenaan in mijn citatielijst. Renate, jij bent echt een liefhebber van muggenonderzoek en dank dat je mij hiermee op weg hebt geholpen.

I got many students to supervise over the years. Thank you all. You kept me sharp, focussed and young. Jacob Beeuwkes en Teun Koelewijn in het bijzonder. Door jullie inzet, creativiteit en doorzettingsvermogen is het gelukt om grote projecten af te ronden, zoals de verwezenlijking van het 3D-trackingsysteem, en het succes dit systeem te draaien in stoffig en vochtig Kenia. Als co-auteur van hoofdstuk 4, 5 en 6 vormt jullie bijdrage het hart van dit proefschrift. Daarnaast was het ook gewoon heel gezellig. Teun K., fijn dat je paranimf wilde zijn. Zo lang stil zitten is echt vreselijk.

Nu hoofdstuk 4 toch ter sprake is gekomen. Dit is echt een teamprestatie geweest. Al met al zijn we 7 jaar in de weer geweest (nee, niet full-time) om e.e.a. af te ronden. Dan reken ik nog niet eens al het voorwerk van mijn voorganger Piet Huisman mee. Ik wil Kees Spoor en Fabrizio Grieco in het bijzonder danken. Voor alle uren die jullie staken in het maken van verbeterslagen.

To all the Mbita point staff members at ICIPE, thanks for hosting me and thanks for being supportive to students like Teun. Richard Mukabana, it was great to finally meet you in Kenya, where the real action is, rather than meeting in cold and windy Wageningen only.

Camille Ponzio, you temporarily said goodbye to the insect-plant group to work with mosquitoes for the project with Helen Pates (Chapter 7). And yes, at some point you had to admit that you liked it! Thanks for your input. Helen, thanks for your trust in taking on this project. I still see it as a good example where fundamental science meets applied sciences.

Zonder insectenkweek geen onderzoek. André en Frans, Leo, Léon. Jullie zijn (waren) de keepers van het team. Onmisbaar, en jullie moeten altijd als eerste de bal uit het net halen wanneer er iets mis gaat. Dank voor alle armen bloed en de uren zweet door het werken in warme klimaatkamers, ook in het weekend.

Team Tupola, Ton, Chris, Eltje, Johan, Bianca, Evert, dank voor al jullie hulp, kritisch meedenken oplossingen te bedenken en vervolgens te maken. 'Jullie' windtunnels vliegen inmiddels de hele wereld over. Sjaak van Brugge noem ik hier ook even, 
want Ton en Sjaak hebben bij de ontwikkeling van het klimaatbeheersingsysteem iets unieks in elkaar gezet.

From Vectorgroup to OneHealthEntomology (OHE). Again, too many names to mention. Sander, I'm looking forward working in this group the coming years. Chantal, I'll miss your great questions and remarks, good luck in Yale. Niels, Jetske, you're gone but most welcome to come back. Alex, you are a great project leader and big fun for the group. Thanks for being paranymph! Tessa, it's incredible that I know you since the time you came in as an overenthusiastic high-school student working (twice) on your 'profielwerkstuk'. Now, you are still overenthustastic, but as a PhD student. Tim, can't you just stay?

The Experimental Zoology group. Our collaboration started years ago with Johan, Sander and Kees. Thanks to your programming skills, Noldus IT managed to release the add-on tool for Ethovision, 'Track 3D'. Lucas, although the market for insect tracking is relatively small, it is great to see that the enthusiasm for entomology projects is still in your blood. Martin and Florian also got infected with the fun of mosquito tracking. I'm looking forward to continue the joined projects, together with Antoine and technical support of Remco.

Sjonge, ik had me voorgenomen vooral niet alle namen te noemen. Blijft toch lastig. Alle vaste staf, dank voor de collegialiteit. Patrick, Hans, Angelique. Fijn hè, al die EBS bestellingen? Joop van Loon, dank voor je humor. Gerard, ik reken jou ook tot de vaste staf. Je bent altijd enorm betrokken bij alles en iedereen. Dank voor het luxe gebak dat je elk jaar meebrengt. Kamergenoten: Jullie hebben het allemaal langer dan 5 jaar met mij uitgehouden. Heel knap! Rieta, op de Binnenhaven hadden we het, naast de geur en herrie van de vlinderkamer, ondanks de hitte in de zomer, maar mét eigen koffiezetapparaat, best goed. Je bent een ster met Excel 'IfElseLookup' functies, en bij het 'Hulstvliegpracticum' zijn we al ontelbaar jaren een goed duo. Léon, op de een of andere manier wilde Rieta toch bij me weg. Maar gelukkig, ook jij bleek een perfecte kamergenoot. We kregen Nina er als bonus bij. Jullie beiden wens ik heel veel succes met de nieuwe richting die jullie zijn ingeslagen. En dank voor de leuke tijd. Pieter, jij leerde mij dit jaar als kamergenoot kennen als iemand die niet wilde praten, maar alleen maar wilde typen. Na het inleveren van de leesversie ging de druk er wat af, en heb ik inmiddels genoeg woorden met je gewisseld om te beseffen dat ik het wederom getroffen heb. Het hele lab mag in zijn handen knijpen met jou als nieuw hoofd van de insectenwkeek. 
PhD's and post-docs come and go. It often feels like a brain drain, but I do understand that you could not all (or want to) stay. Ernst-Jan, Krijn, David, Gilian, Fedor,.. ja iedereen natuurlijk, maar jullie brachten ook op persoonlijk vlak een hoop leuke inspiratie. Ernst-Jan in het bijzonder, ondanks dat hij veel te kort leefde. Gelukkig heeft hij heel veel moois achtergelaten voor familie, vrienden en collega wetenschappers. Zijn werk is nog steeds inspirerend en we putten daar nog steeds uit. Kijk anders maar naar de referentielijst in dit proefschrift $:)$. We won't forget. Current PhD group, thanks for allowing me at the PhD-lunch. First you thought I was a spy from Marcel, but no. I enjoyed the diversity of talks and discussions we had. You are a bunch of extremely intelligent people. Yavanna, thanks for taking care of the layout of this thesis. You can now fully focus on yours. The PhD's and teachers with whom I had a great time in South-Africa during the course 'The Science of Conservation'. Thanks to you all, and thanks Lennart \& Claudius from PE\&RC for organizing it.

Alex, Ana, Cindy, oh no! How can I ever name all the people that give (or gave) colour and vibrance to the group. Without people like you, the office would be full of Ento nerds ;-) . Futurum!!! app-group, you know what I mean.

Françoise, ik mocht jou (aldus Willem) nooit in een dankwoord noemen voor de laatste check voor het gebruikte Engels in manuscripten. Ha, dan nu toch! Soms dachten Willem en ik dat we een artikel bijna konden indienen, maar kwam jij op de valreep nog met een hele lijst verbeterpunten. Enorm bedankt hiervoor.

Hans en Annelies, dit proefschrift had al veel eerder af kunnen zijn. Maar het werken in de tuin vind ik zo leuk dat ik een werkdag in Wageningen heb ingeleverd. Dank dat jullie het aandurfden met ons het huis en grond te delen.

BOS-band mannen: Dank voor de wekelijkse uren ontspanning, mooie muziek en lol die we maakten. Vikingen: ledereen is uniek, maar jullie zijn wel heel bijzonder. Inmiddels weten veel collega's dat ik $1 x$ per jaar even naar Tsjechië 'moet'. Oh ja, over het nut van de mug: Dieren zijn hier niet op aarde om nuttig te zijn, maar slechts een (soms best fraai) resultaat van evolutie. Dus die vraag hoeven jullie me niet meer te stellen.

Beverwerkroep, nu beter bekend als Knot(s) ploeg: Jullie passie voor biologie is uniek en aanstekelijk. Ja, jullie zijn knots om in ruil voor slechts 1 (of 2) appeltaarten een heel weekend hout te verslepen. Bedankt Nienke Beintema, misschien wel de beste wetenschapsjournalist van Nederland (deze stelling valt moeilijk te checken), dat je de Nederlandse samenvatting in dit proefschrift voor een breder publiek leesbaarder hebt helpen maken. 
Ouders, Martien en Mea, dank dat jullie me dwongen naar boven te gaan om huiswerk te maken, al keek ik daar natuurlijk liever uit het raam dan dat ik m'n hoofd in de boeken stopte. De lol in leren kreeg ik pas toen ik kon doen wat ik wilde doen. Ik was pas 17 toen ik Diermanagement ging studeren. Ik kreeg van jullie het vertrouwen om op kamers te gaan en er wat moois van te maken. Het leven op de boerderij heeft me een breed scala aan inzichten en praktische vaardigheden opgeleverd waar ik nog altijd veel aan heb. Het kritisch 'op alles' zijn lijkt erfelijk. Rian en Hugo, dank voor jullie wijze inzichten door de jaren heen. Schoonfamilie incluis, wat fijn dat de families compleet zijn en dank voor jullie betrokkenheid. Ike, bedankt voor het haastig nemen van de foto!

Wil Ueberbach, jij bent verreweg het beste wat Markplaats ooit te bieden heeft gehad. Het fijne thuiskomen en het geluk van Anna en Teun is voor een groot deel aan jou te danken. Als 'gastouder' ben je echt een extra ouder voor ze geweest, alleen heb je veel meer talent hiervoor dan wijzelf. Bedankt!

Lieve Annemarieke, je hebt mijn niveau door de jaren heen aardig omhoog weten te krikken. Niet alleen met feedback op woorden of zinnen die af en toe, vooral de laatste weken, via e-mail heen en weer vlogen. Het begon al tijdens ons gezamenlijk afstuderen in Cambridge. Ik ben blij dat we onze beide promotietrajecten nooit als doel op zich hebben gezien, maar meer als bijkomend onderdeel van ons werk. Ons huisje, boompje, beestje model is niet van de familie doorsnee, en juist daarom zo leuk. Ik hoop dat we er nog lang samen van kunnen genieten.

Tot slot, lieve Anna en Teun, veel ouders werken eerst aan de geboorte van hun proefschrift en daarna pas aan de geboorte van hun kinderen. Zonde! Jullie hebben de afgelopen jaren voor heel veel plezier en ontspanning gezorgd. Af en toe moest ik voor mijn werk op reis, maar gelukkig kon ik verder wel veel thuis zijn om jullie te zien opgroeien. Samen aan de voorkant van dit proefschrift werken, tijdens een potje dammen. Wat een geluk! Wel jammer dat ik toen weer van Teun verloor... Ik hoop dat jullie veel vertrouwen in jezelf blijven houden. Onthoud goed dat wanneer je écht graag iets wilt, het ook lukt. 


\section{Curriculum Vitae}

Jeroen Spitzen was born in 1978 in Wolvega, the Netherlands and grew up near Oldemarkt on an organic dairy farm near National Park 'The Weerribben'. Here he developed an interest in wildlife and ecology.

In 1995 he started studying for a bachelor degree in Animal Management at the Van Hall Institute in Leeuwarden. He went abroad for all his traineeships and a graduation project. First to Norway, to study the behaviour of greylag geese in relation to agricultural practices. Later to New

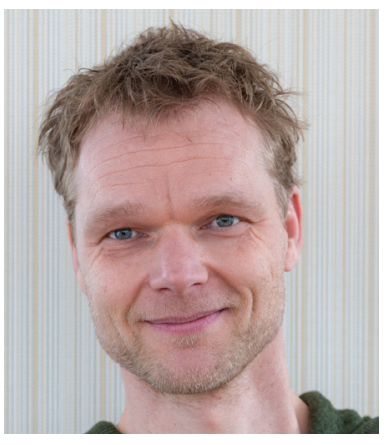
Zealand, to study the effect of predator control on local skink populations. This traineeship resulted in a temporary contract as a technician at the Department of Conservation in Dunedin, New Zealand. His thesis project was conducted at the University of Cambridge, United Kingdom, where he studied human-cattle interactions in city parks.

In 2002 he started as a technician at the Laboratory of Entomology, Wageningen University \& Research, to work with Prof. Arnold van Huis and Prof. Willem Takken. He started to work on the behavioural ecology of malaria mosquitoes and developed an automated tracking system in collaboration with Noldus IT and the Experimental Zoology group of Wageningen University \& Research.

He will continue working as a research-/teaching associate at the Laboratory of Entomology and proceed with his work on the flight behaviour of insects.

Jeroen lives together with his wife and children in a farmhouse near Winssen, where he enjoys chopping wood, growing their own fruits and vegetables while observing local wildlife. 


\section{List of publications}

\section{Refereed articles in a journal}

Spitzen, J., and Takken, W. (2018). Keeping track of mosquitoes: a review of tools to track, record and analyse mosquito flight. Parasites \& Vectors, 11, 123.

Muijres, F.T., Chang, S.W., Van Veen, W.G., Spitzen, J., Biemans, B.T., Koehl, M.A.R., and Dudley, R. (2017). Escaping blood-fed malaria mosquitoes minimize tactile detection without compromising on take-off speed. Journal of Experimental Biology, 220, 3751-3762.

Spitzen, J., Koelewijn, T., Mukabana, W.R., and Takken, W. (2017). Effect of insecticide-treated bed nets on house-entry by malaria mosquitoes: The flight response recorded in a semi-field study in Kenya. Acta Tropica, 172, 180-185.

Spitzen, J., Koelewijn, T., Mukabana, W.R., and Takken, W. (2016). Visualization of house-entry behaviour of malaria mosquitoes. Malaria Journal, 15:233.

Spitzen, J., Ponzio, C., Koenraadt, C.J.M., Pates Jamet, H.V., and Takken, W. (2014). Absence of close-range excitorepellent effects in malaria mosquitoes exposed to deltamethrin-treated bed nets. American Journal of Tropical Medicine and Hygiene, $90,1124-1132$.

Spitzen, J., Spoor, C.W., Grieco, F., ter Braak, C., Beeuwkes, J., van Brugge, S.P., Kranenbarg, S., Noldus, L.P.J.J., van Leeuwen, J.L., and Takken, W. (2013). A 3D analysis of flight behavior of Anopheles gambiae sensu stricto malaria mosquitoes in response to human odor and heat. PloS ONE, 8, e62995.

van Houte, S., Ros, V.I., Mastenbroek, T.G., Vendrig, N.J., Hoover, K., Spitzen, J., and van Oers, M.M. (2012). Protein tyrosine phosphatase-induced hyperactivity is a conserved strategy of a subset of baculoviruses to manipulate lepidopteran host behavior. PloS ONE, 7, e46933.

Smallegange, R.C., Schmied, W.H., van Roey, K.J., Verhulst, N.O., Spitzen, J., Mukabana, W.R., and Takken, W. (2010). Sugar-fermenting yeast as an organic source of carbon dioxide to attract the malaria mosquito Anopheles gambiae. Malaria Journal, 9, 292. 
Spitzen-van der Sluijs, A., Spitzen, J., Houston, D., and Stumpel, A.H.P. (2009). Skink predation by hedgehogs at Macraes Flat, Otago, New Zealand. New Zealand Journal of Ecology, 33, 205-207.

Gassner, F., P. Verbaarschot, R. C. Smallegange, J. Spitzen, S. E. Van Wieren, and Takken, W. (2008). Variations in Ixodes ricinus density and Borrelia infections associated with cattle introduced into a woodland in the Netherlands. Applied Environmental Microbiology, 74, 7138-7144.

Spitzen, J., Smallegange, R.C., and Takken, W. (2008). Effect of human odours and positioning of $\mathrm{CO} 2$ release point on trap catches of the malaria mosquito Anopheles gambiae sensu stricto in an olfactometer. Physiological Entomology, 33, 116-122.

Qiu, Y.T., Smallegange, R.C., Ter, B.C., Spitzen, J., Van Loon, J.J., Jawara, M., Milligan, P., Galimard, A.M., Van Beek, T.A., Knols, B.G., et al. (2007). Attractiveness of MM-X traps baited with human or synthetic odor to mosquitoes (Diptera: Culicidae) in The Gambia. Journal of Medical Entomology, 44, 970-983.

Spitzen, J., and Van Huis, A. (2005). Effect of host quality of Callosobruchus maculatus (Coleoptera: Bruchidae) on performance of the egg parasitoid Uscana lariophaga (Hymenoptera: Trichogrammatidae). Bulletin of Entomological Research, 95, 341-347.

Hagen, K., van der Sluijs, A., Spitzen, J., and Broom, D.M. (2001). Interactions between cattle and people on city-centre commons in Cambridge, UK. Current Research in Applied Ethology (Darmstadt: KTBL), 403, 96-103.

\section{Book chapters}

Verhulst, N.O., Boulanger, N., and Spitzen, J. (2018). Impact of skin microbiome on attractiveness to arthropod vectors and pathogen transmission. In Skin and Arthropod Vectors, N. Boulanger, ed. (Academic Press, Elsevier), 55-82.

Qiu, Y.T., Spitzen, J., Smallegange, R.C., and Knols, B.G.J. eds. (2007). Monitoring systems for adult insect pests and disease vectors, In Emerging pests and vectorborne diseases in Europe, (Vol.1) W. Takken \& B.G.J. Knols, ed. (Wageningen Academic Publishers), 329-353.

Takken, W., Rooij, E.M.A., van, Verhulst, N.O., Jacobs, F., Huijben, S., Beeuwkes, J., Groot, N., Vos, V.C.A., Spitzen, J., Heutink, R.C.G., et al. (2007). Bluetongue: 
an emerging vector-borne disease outbreak in North-western Europe. In Emerging pests and vector-borne diseases in Europe, (Vol.1) W. Takken \& B.G.J. Knols, ed. (Wageningen Academic Publishers), 113-121.

\section{Non-refereed conference papers}

Spitzen, J., C.W. Spoor, S. Kranenbarg, J. Beeuwkes, F. Grieco, L.P.J.J. Noldus, J.L. van Leeuwen, and Takken, W. (2008). Track3D: Visualization and flight track analysis of Anopheles gambiae s.s. mosquitoes In Measuring Behavior 2008, M.R.B. A.J. Spink, N.D. Bogers, F. Grieco, L.W.S. Loijens, L.P.J.J. Noldus, G. Smit, and P.H. Zimmerman, ed., 133-135.

Beeuwkes, J., Spitzen, J., Spoor, C.W., Leeuwen, J.L., van, and Takken, W. (2008). 3-D flight behaviour of the malaria mosquito Anopheles gambiae s.s. inside an odour plume. Proceedings of the Netherlands Entomological Society Meeting, 19, 137-146.

Spitzen, J., and Takken, W. (2005). Malaria mosquito rearing - maintaining quality and quantity of laboratory-reared insects. Proceedings of the Netherlands Entomological Society Meeting, 16, 95-100.

\section{In preparation}

Cribellier, A., Van Erp, J.A., Hiscox, A., Lankheet, M.J., Van Leeuwen, J.L., Spitzen, J., and Muijres, F.T. (submitted). Flight dynamics of malaria mosquitoes around odour-baited traps: capture and escape dynamics.

Mburu, M.M., Juurlink, M., Spitzen, J., Hiscox, A., Mzilahowa, T., Takken, W., and McCann, R.S. (submitted). Impact of partially and fully closed eaves on house entry rates by mosquitoes. 


\section{PE\&RC Training and Education Statement}

With the training and education activities listed below the PhD candidate has complied with the requirements set by the C.T. de Wit Graduate School for Production Ecology and Resource Conservation (PE\&RC) which comprises of a minimum total of 32 ECTS (= 22 weeks of activities)

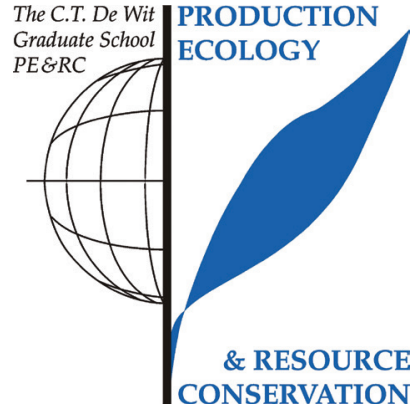

Review of literature (4.5 ECTS)

- Keeping track of mosquitoes: a review of tools to track, record and analyse mosquito flight

\section{Writing of project proposal (1 ECTS)}

- Analysis of flight behaviour of the malaria mosquito An. gambiae s.s. (2014)

\section{Post-graduate courses (3.2 ECTS)}

- The science of conservation; PE\&RC (2016)

\section{Laboratory training and working visits (6.1 ECTS)}

- Outdoor trapping malaria mosquitoes using odour baited traps

- Exchange meeting on behavioural ecology and insect rearing

- Filming house entry behaviour of malaria mosquitoes

- Work visit, collaboration on behavioural recording in the semi-field

Invited review of (unpublished) journal manuscript (7 ECTS)

- J. of Interface Science and Techn.: mosquito flight behaviour (2013)

- J. Experimental Biology: malaria mosquito responses to sweat (2014)

- J. Experimental Biology: carbon dioxide detection in malaria mosquitoes (2015)

- Parasites \& Vectors: mosquito flight behaviour (2016)

- Malaria Journal: mass rearing of mosquitoes, quality (2016)

- Malaria Journal: mosquito flight behaviour (2017)

- Parasites \& Vectors: mosquito flight behaviour, screen traps (2017)

\section{Deficiency, refresh, brush-up courses (3 ECTS)}

- Ecological methods (2009)

\section{Competence strengthening / skills courses (2.2 ECTS)}

- Project and Time Management (P\&TM)

- Data Management (DM)

- PhD Workshop carousel

\section{PE\&RC Annual meetings, seminars and the PE\&RC weekend (0.9 ECTS)}

- $3^{\text {rd }}$ Wageningen PhD symposium (2016)

- NERN Meeting (2015)

- PE\&RC Day (2015)

\section{Discussion groups / local seminars / other scientific meetings (8.9 ECTS)}

- Nederlandse entomologendag (2002-2017)

- Vector group (2012-2014)

- WEES Masterclass \& seminar Mike Begon WEES Masterclass Peter Billingsley (2013, 2015)

- Plant seminars (2013-2015) 


\section{Education statement}

- Ecology of infectious diseases in natural populations symposium $(2014,2016)$

- Tick tactics symposium (2016)

International symposia, workshops and conferences (9.8 ECTS)

- Measuring behaviour; oral presentations (2005, 2008)

- American Society of Tropical Medicine annual meeting; oral presentations (2010-

2011)

- Society of Vector Ecology workshop; oral presentation (2016)

\section{Lecturing / Supervision of practicals / tutorials (3 ECTS)}

- Population dynamics of insects

\section{Supervision of 11 MSc students}

- Mosquito host-seeking behaviour

- Mosquito oviposition behaviour

- Automated tracking of insects 
Thesis layout: Yavanna Aartsma (www.amongstwildflowers.com) Cover design: Jeroen Spitzen Drawing front: Anna \& Teun Spitzen Software back: Copyright 2018, tagxedo.com

Printed by GVO drukkers \& vormgevers, Ede, The Netherlands 


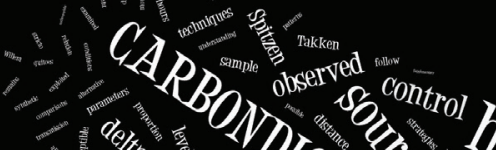

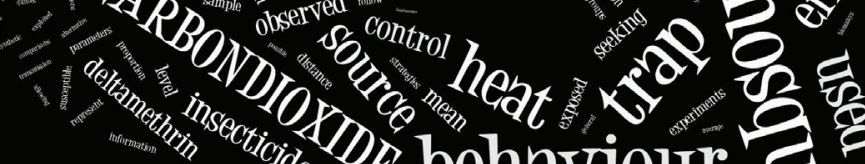
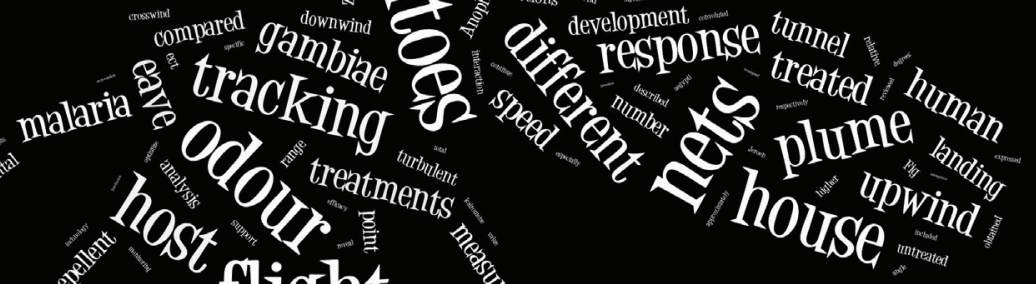